\title{
Geohydrologic and Ground-Water-Quality Data for Stratified-Drift Aquifers in the Exeter, Lamprey, and Oyster River Basins, Southeastern New Hampshire
}

U.S. GEOLOGICAL SURVEY

Open-File Report 92-95

Prepared in cooperation with the

NEW HAMPSHIRE DEPARTMENT OF ENVIRONMENTAL SERVICES, WATER RESOURCES DIVISION 


\section{GEOHYDROLOGIC AND GROUND-WATER-QUALITY DATA FOR STRATIFIED-DRIFT AQUIFTERS IN THE EXETER, LAMPREY, AND OYSTER RIVER BASINS, SOUTHEASTERN NEW HAMPSHIRE}

By Richard Bridge Moore

\section{U.S. GEOLOGICAL SURVEY}

Open-File Report 92-95

Prepared in cooperation with the NEW HAMPSHIRE DEPARTMENT OF ENVIRONMENTAL SERVICES, WATER RESOURCES DIVISION

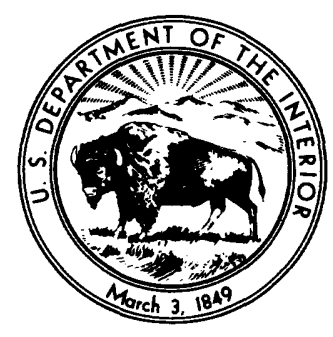

Bow, New Hampshire 1992 


\title{
U.S. DEPARTMENT OF THE INTERIOR
}

\author{
MANUEL LUJAN JR., Secretary
}

\section{U.S. GEOLOGICAL SURVEY}

Dallas L. Peck, Director

For additional information, write to:

\section{U.S. Geological Survey}

Water Resources Division

525 Clinton Street

Bow, NH 03304
Copies of this report may be purchased from:

U.S. Geological Survey

Books and Open-File Reports Section

Box 25425, Federal Center

Denver, CO 80225 


\section{CONTENTS}

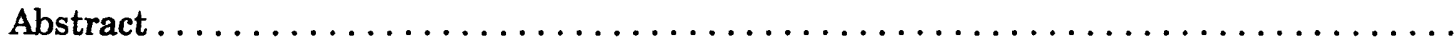

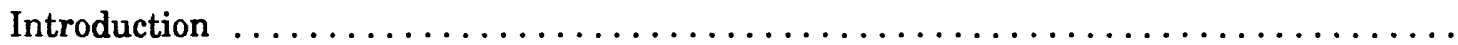

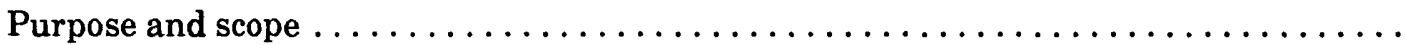

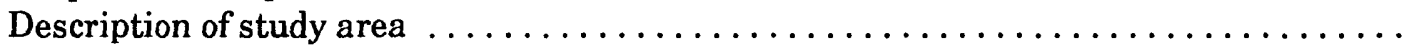

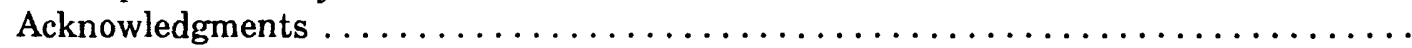

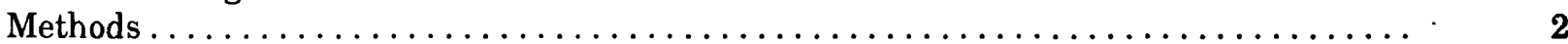

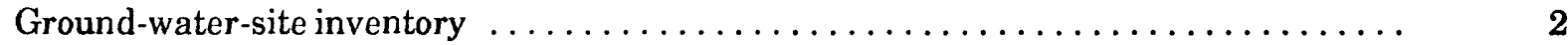

Test-hole drilling and installation of observation wells $\ldots \ldots \ldots \ldots \ldots \ldots \ldots \ldots \ldots .2$

Ground-water-level measurements ...........................

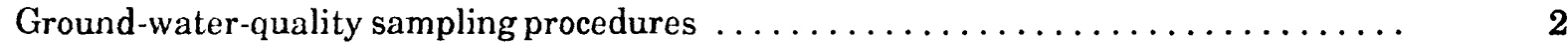

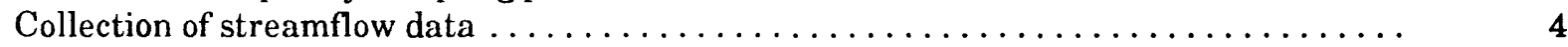

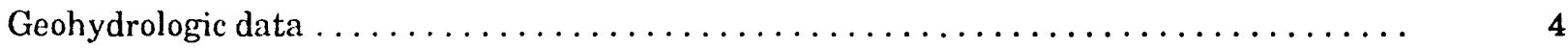

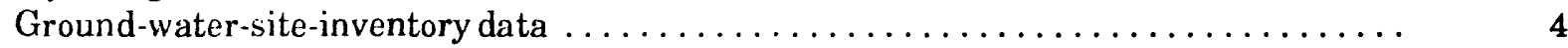

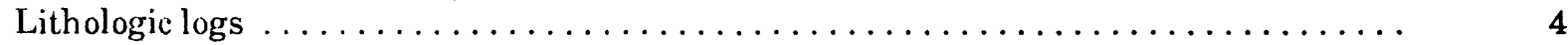

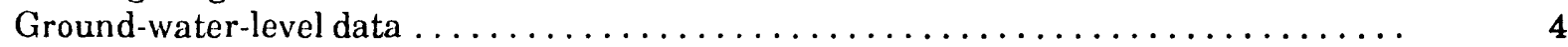

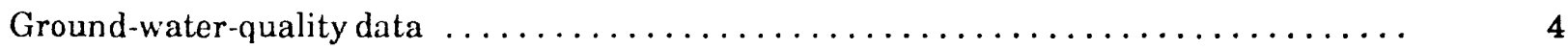

Selected references $\ldots \ldots \ldots \ldots \ldots \ldots \ldots \ldots \ldots \ldots \ldots \ldots \ldots \ldots \ldots \ldots$

\section{PLATES}

[Plates are in back of map jacket.]

Plate 1-4: Maps showing geohydrologic and water-quality-data collection locations in the:

1. northwestern quadrant of study area.

2. northeastern quadrant of study area.

3. southwestern quadrant of study area.

4. southeastern quadrant of study area. 


\section{ILLUSTRATIONS}

Page

Figure 1. Index map showing location of the Exeter, Lamprey, and Oyster River basins in southeastern New Hampshire $\ldots \ldots \ldots \ldots \ldots \ldots \ldots \ldots \ldots \ldots \ldots$

2-21. Ground-water-level hydrographs

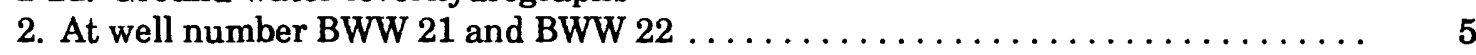

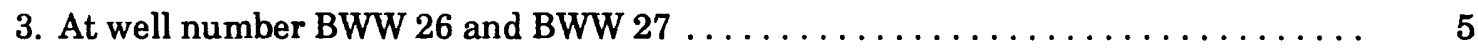

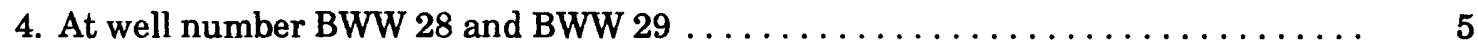

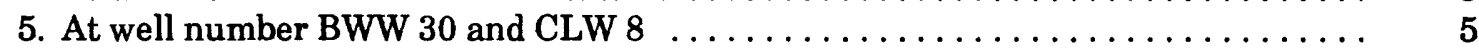

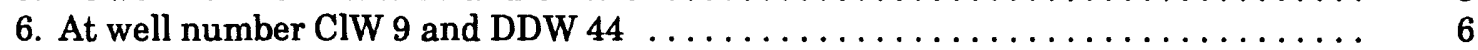

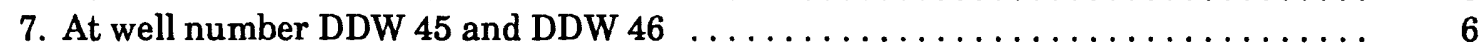

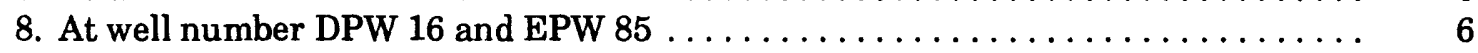

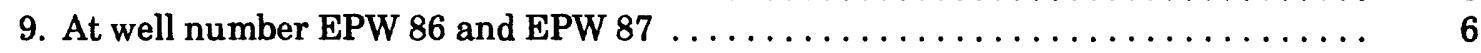

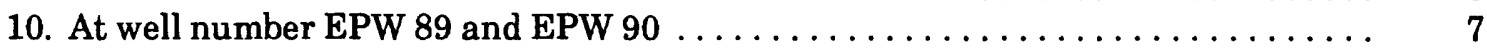

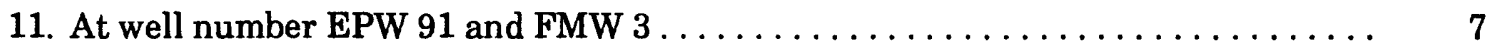

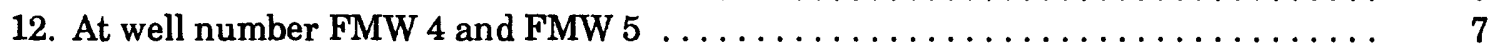

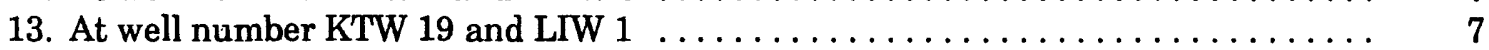

14. At well number MAW 56 and NGW $12 \ldots \ldots \ldots \ldots \ldots \ldots \ldots \ldots \ldots \ldots$

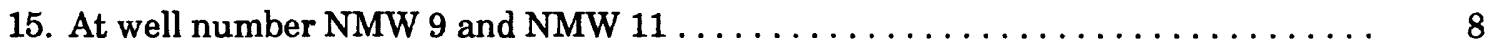

16. At well number RBW 8 and RBW $9 \ldots \ldots \ldots \ldots \ldots \ldots \ldots \ldots \ldots \ldots \ldots$

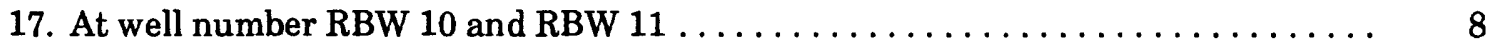

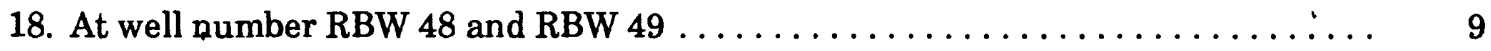

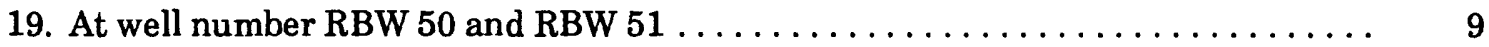

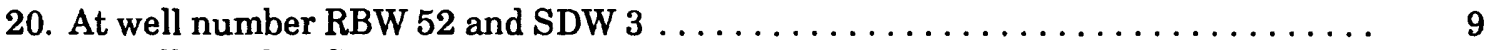

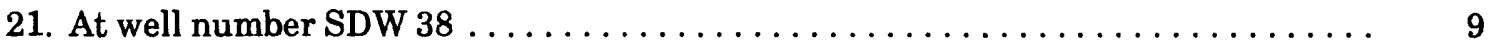

22. Map showing ground-water-quality sampling locations $\ldots \ldots \ldots \ldots \ldots \ldots \ldots \ldots$

\section{TABLES}

Table 1. Two-letter town codes used as prefixes in the numbering system

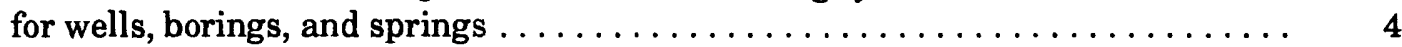

2. Description of wells, borings, and springs $\ldots \ldots \ldots \ldots \ldots \ldots \ldots \ldots \ldots \ldots \ldots \ldots \ldots$

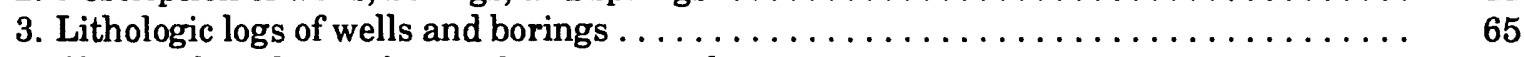

4. Chemical analyses of ground-water samples $\ldots \ldots \ldots \ldots \ldots \ldots \ldots \ldots \ldots \ldots \ldots \ldots$

5. Drinking-water regulations and recommended limits $\ldots \ldots \ldots \ldots \ldots \ldots \ldots \ldots$ 


\section{CONVERSION FACTORS, VERTICAL DATUM AND ABBREVIATED WATER-QUALITY UNITS}

Multiply

inch (in.)

foot (ft)

mile (mi)

square mile (mi )
By

To obtain millimeter

0.3048

meter

1.609

kilometer

2.59

Sea Level: In this report, "sea level" refers to the National Geodetic Vertical Datum of 1929--a geodetic datum derived from a general adjustment of the first-order level nets of the United States and Canada, formerly called Sea Level Datum of 1929.

Chemical constituents and water temperature are given in SI units. Chemical concentration is given in milligrams per liter $(\mathrm{mg} / \mathrm{L})$ or micrograms per liter $(\mu \mathrm{g} / \mathrm{L})$. Milligrams per liter is a unit expressing the concentration of chemical constituents in solution as weight (milligrams) of solute per unit volume (liter) of water; $1,000 \mu \mathrm{g} / \mathrm{L}$ (micrograms per liter) is equivalent to $1 \mathrm{mg} / \mathrm{L}$ (milligrams per liter).

Water temperature is given in degrees Celsius $\left({ }^{\circ} \mathrm{C}\right)$, which can be converted to degrees Fahrenheit $\left({ }^{\circ} \mathrm{F}\right)$ by the following equation ${ }^{\circ} \mathrm{F}=1.8\left({ }^{\circ} \mathrm{C}\right)+32$. 


\title{
Geohydrologic and Ground-Water-Quality Data for the Stratified-Drift Aquifers in the Exeter, Lamprey, and Oyster River Basins, Southeastern New Hampshire
}

\author{
By Richard Bridge Moore
}

\begin{abstract}
This report presents geohydrologic and ground-waterquality data collected for a study of stratified-drift aquifers located within the Exeter, Lamprey, and Oyster River basins in southeastern New Hampshire. The study was done in cooperation with the New Hamp. shire Department of Environmental Services, Water Resources Division.

The data include information on more than 1,200 well-inventory sites and 57 test holes drilled specifically for this study. Water-level measurements were recorded for U.S. Geological Survey observation wells measured during the course of the project. Water-quality analyses are presented for 35 observation wells, 2 springs, and 2 municipal-supply well systems. Waterquality characteristics measured in the field included temperature, specific conductance, and pH. Samples were analyzed in the laboratory for nutrients, common inorganic anions and cations, and selected volatile organic compounds. The plates show the locations of all data-collection sites.
\end{abstract}

\section{INTRODUCTION}

Stratified-drift aquifers within the Exeter, Lamprey, and Oyster River basins in southeastern New Hampshire are an important water resource for the region. A quantitative study of this resource was initiated in 1983 by the U.S. Geological Survey (USGS) in cooper- ation with the New Hampshire Department of Environmental Services (NHDES), Water Resources Division.

The objectives of the study are to (1) describe the hydrologic and geologic characteristics of the stratified-drift aquifers within the study area, (2) present a technique for evaluating the hydrologic impact of ground-water development, and (3) assess the quality of the ground water in the stratified-drift aquifers.

\section{Purpose and Scope}

The purpose of this report is to provide basic data compiled during the study to those individuals and municipalities concerned with water-resource management. Information in this report includes: well-inventory data on the more than 1,200 inventory sites and 57 test holes drilled for this study, test hole logs, ground-water-level data for USGS observation wells measured during the course of the projec, and groundwater-quality data for samples taken from 35 observation wells, 2 springs, and 2 municipal supply-well systems.

A companion interpretive report presenting the conclusions drawn from the study was completed in 1988 and is titled "Geohydrology and Water Quality of Stratified-Drift Aquifers in the Exeter, Lamprey, and Oyster River Basins, Southeastern New Hampshire" (Moore, 1990). 


\section{Description of the Study Area}

The study area is in southeastern New Hampshire (fig. 1), and covers $351 \mathrm{mi}$, of which approximately 56 $\mathrm{mi}$ are underlain by stratified-drift aquifers. Many towns in the study area with municipal-water supplies rely partially, if not completely, on ground water for their water needs.

The stratified-drift aquifers in the western and central sections of the study area are valley-fill aquifers and consist of eskers, kames, kame terraces, outwash, and outwash deltas. In the eastern section of the study area they are predominantly deltaic aquifers, some of which are confined beneath fine-grained silts and clays. A major source of data for delineating aquifer boundaries and the extent of the silts and clays in southeastern New Hampshire was 1:24,000-scale, surficial geologic maps produced by the Cooperative Geologic Mapping Program (COGEOMAP), a cooperative program between the New Hampshire Department of Environmental Services, Office of the State Geologist, and the Geologic Division of the USGS. These surficial geologic maps are the Kingston (Earl, 1983), Candia (Gephart, 1985a), Derry (Gephart, 1985b), and Sandown (Gephart, 1987) quadrangles. In addition, preliminary surficial geologic (COGEOMAP) maps of the Portsmouth; Exeter, N.H.Mass.; Newmarket; and Epping quadrangles were made available by the Office of the New Hampshire State Geologist.

\section{Acknowledgments}

The author would like to thank the many State, Federal, and municipal officials, residents, well contractors, and consulting firms who provided data for this study. Included are personnel from the Office of the State Geologist, the New Hampshire Water Resources Division, the University of New Hampshire, and the U.S. Soil Conservation Service. The author would also like to express his appreciation to all those who assisted in data collection, especially Timothy Fagan and Diana Morgan. Thanks go to Rick Chormann of the New Hampshire Water Resources Division for assisting in the development of computer programs to transfer data automatically from their computer data base to the USGS's data base.

\section{METHODS}

\section{Ground-Water-Site Inventory}

Well, test boring, and spring information was collected from owners of domestic wells, municipalities, consultant reports, previous USGS reports (Bradley and Petersen, 1962; Cotton, 1987) and from well records provided by the New Hampshire Department of Environmental Services, Water Resources Division. Additional information was provided by the New Hampshire Department of Transportation pertaining to bridge and highway borings.

\section{Test Hole Drilling and Installation of Observation Wells}

Fifty-seven test holes were drilled to obtain information on sediment particle-size, lithology, depth to water table, depth to bedrock, and samples for waterquality analysis.

Adrill rig equipped with hollow-stem augers was used for test-hole drilling. Lithologic samples above the water table were brought to the surface by rotation of the augers. Below the water table, a split-spoon sampler was used to collect lithologic samples ahead of the drill stem. Thirty-eight wells were drilled, cased, and screened with 2 -inch-diameter schedule-40 polyvinyl chloride (PVC). Water samples were collected and water-level measurements were made. All PVC connections were threaded to avoid possible contamination of water samples by the volatile organic compounds contained in PVC glue. The screens used were either $2.0 \mathrm{ft}$ or $5.0 \mathrm{ft}$ in length with slot openings ranging from 0.01 to $0.006 \mathrm{in}$. in width. The casing and screen were placed inside the hollow-stem auger and the hole was allowed to collapse as the drill stem was withdrawn.

\section{Ground-Water Level Measurements}

Periodic water levels were measured using a steel tape accurate to $\pm 0.01 \mathrm{ft}$ at USGS observation wells.

\section{Ground-Water-Quality Sampling Procedures}

Ground-water samples were collected in August and November 1984 and November and December 1985 for analysis of common inorganic, organic, and volatile 


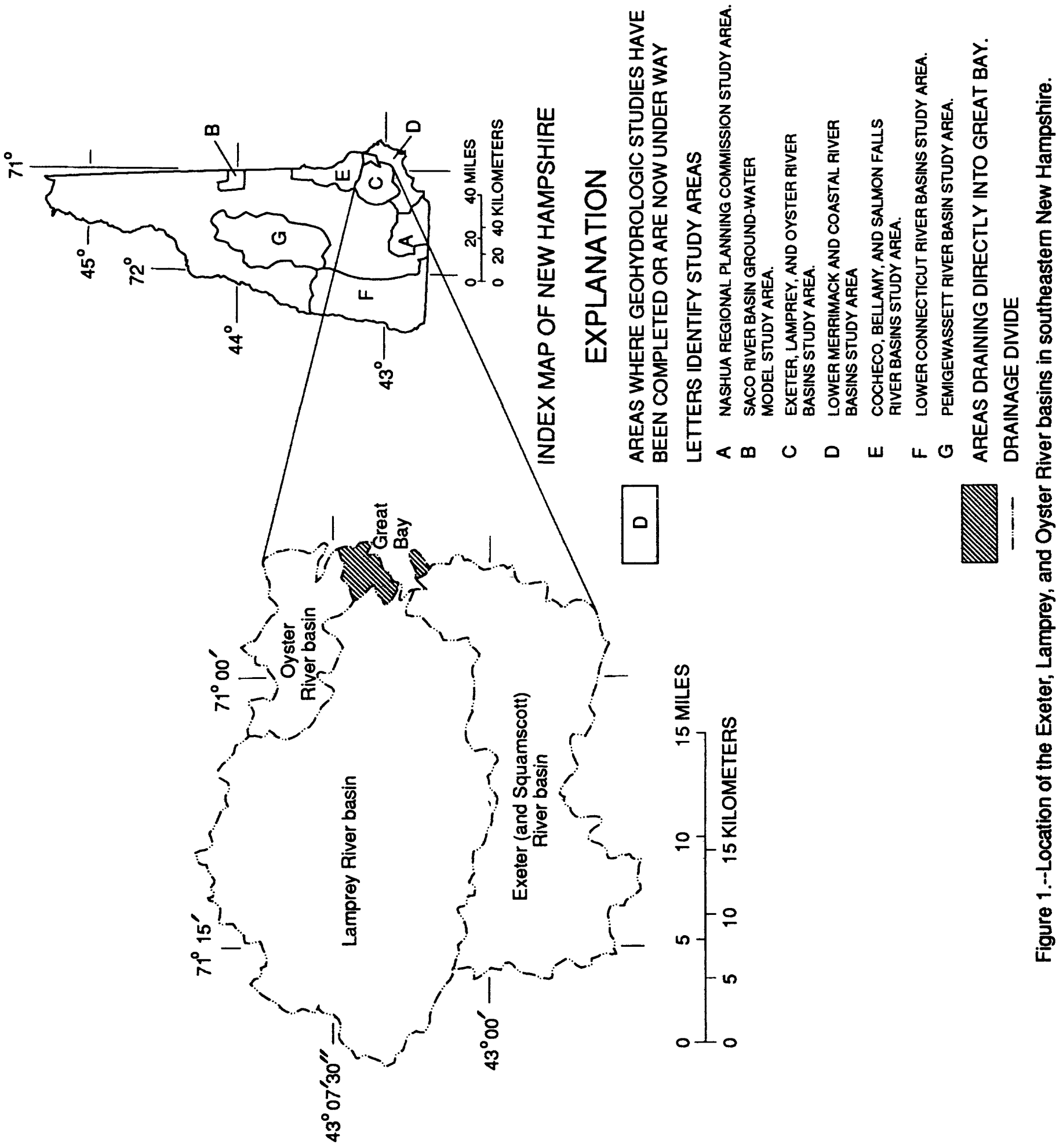


organic constituents at selected USGS wells and municipal wells.

All ground-water samples were analyzed by the USGS Central Laboratories in Doraville, Georgia. Methods for collecting and analyzing the samples are described by Fishman and Freidman (1985). The sampling procedure varied with the source of the water sampled. The municipal wells sampled (NMW-6 and SSW-10) are used continuously so evacuation of water prior to sampling was unnecessary. Furthermore, because water from the municipal wells is treated, care was taken to just sample untreated water. At USGS observation wells, a minimum of 3 times the volume of water in each well was evacuated before the well was sampled, and usually 10 times the volume of the water in the well casing was evacuated before sampling. Specific conductance and temperature readings were stabilized during pumping before sampling. This procedure was followed to ensure that representative samples of ground water were obtained.

\section{Collection of Streamflow Data}

Seepage runs were conducted on unregulated streams within the area and the results are published in Blackey (1987).

Procedures used for the collection of streamflow data are described by Rantz and others (1982a,b).

\section{GEOHYDROLOGIC DATA}

The geohydrologic data presented include well inventory data, lithologic logs, water level, and groundwater-quality data.

\section{Ground-Water-Site-Inventory Data}

Local numbers assigned to wells, test borings, and springs consist of a two-letter town designation (table 1 ), a supplemental letter designation (" $A$ " for borings done for hydrologic purposes with no casing set, "B" for borings done primarily for constructional purposes, "S" for springs, and "W" for all wells in which a casing was set), and a sequential number within each town. For example the first well in the town of Lee is LIW-1. Site inventory data are presented in table 2; locations for the data-collection sites are shown on plates 1-4.
Table 1.--Two-letter town codes used as prefixes in the numbering system for wells, borings, and springs

\begin{tabular}{ll|ll}
\hline \multicolumn{1}{c}{ Town } & $\begin{array}{c}\text { Two- } \\
\text { letter } \\
\text { code }\end{array}$ & \multicolumn{1}{c}{ Town } & $\begin{array}{r}\text { Two- } \\
\text { letter } \\
\text { code }\end{array}$ \\
\hline Barrington & BB & Hampstead & HD \\
Brentwood & BW & Hampton Falls & HF \\
Candia & CD & Kensington & KF \\
Chester & CL & Kingston & KT \\
Danville & DC & Lee & LI \\
Deerfield & DD & Madbury & MA \\
Dover & DJ & Newfields & NG \\
Durham & DP & Newmarket & NM \\
East Kingston & EA & Nottingham & NX \\
Epping & EP & Raymond & RB \\
Exeter & EX & Sandown & SD \\
Fremont & FM & Stratham & SS \\
\hline
\end{tabular}

\section{Lithologic Logs}

Lithologic logs of selected wells and test holes are summarized in table 3.

\section{Ground-Water-Level Data}

Ground-water levels measured periodically at $39 \mathrm{ob}-$ servation wells are presented in figures 2-21. Locations of the wells are shown on plates 1-4.

\section{GROUND-WATER-QUALITY DATA}

Chemical analysis of ground water from 35 observation wells, 2 municipal wells, and 2 springs are given in table 4 and locations of sampling sites are shown on plates 1-4 and on figure 22 .

Primary and secondary drinking-water regulations, set by the U.S. Environmental Protection Agency (1988a, 1988b) and the New Hampshire Water Supply Engineering Bureau (written commun., 1988) are presented in table 5 . 


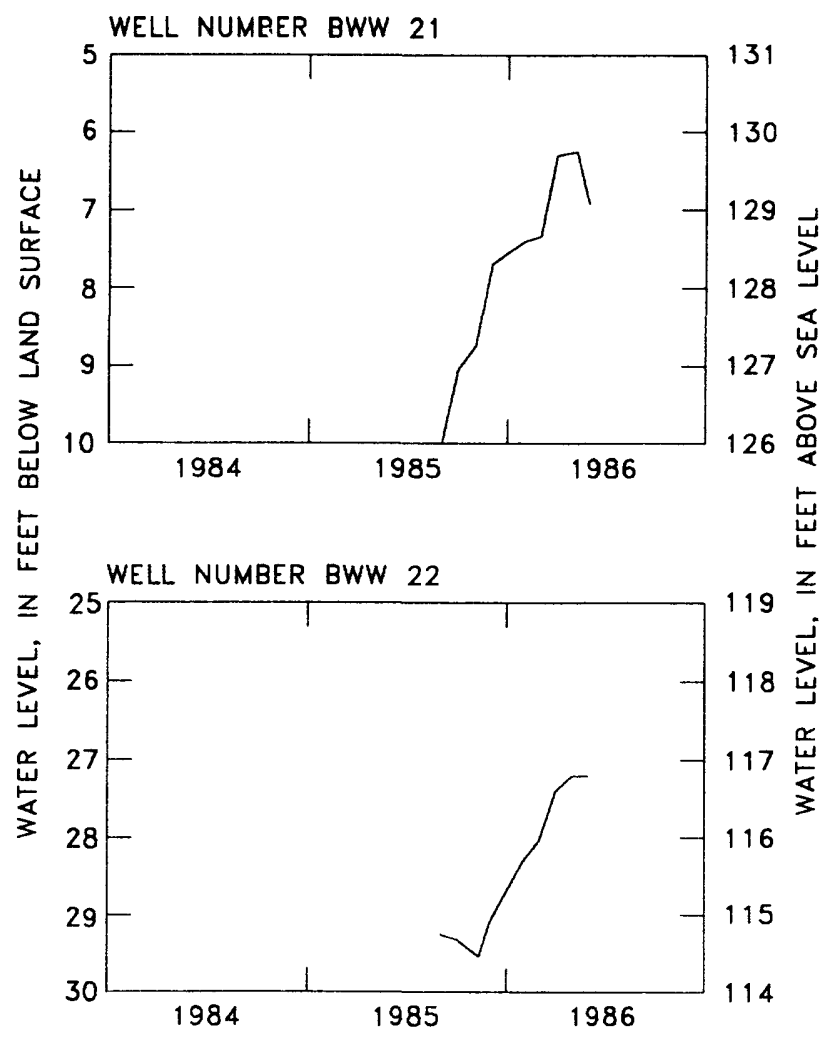

Figure 2.-Periodic water levels at well number BWW21 and BWW 22.

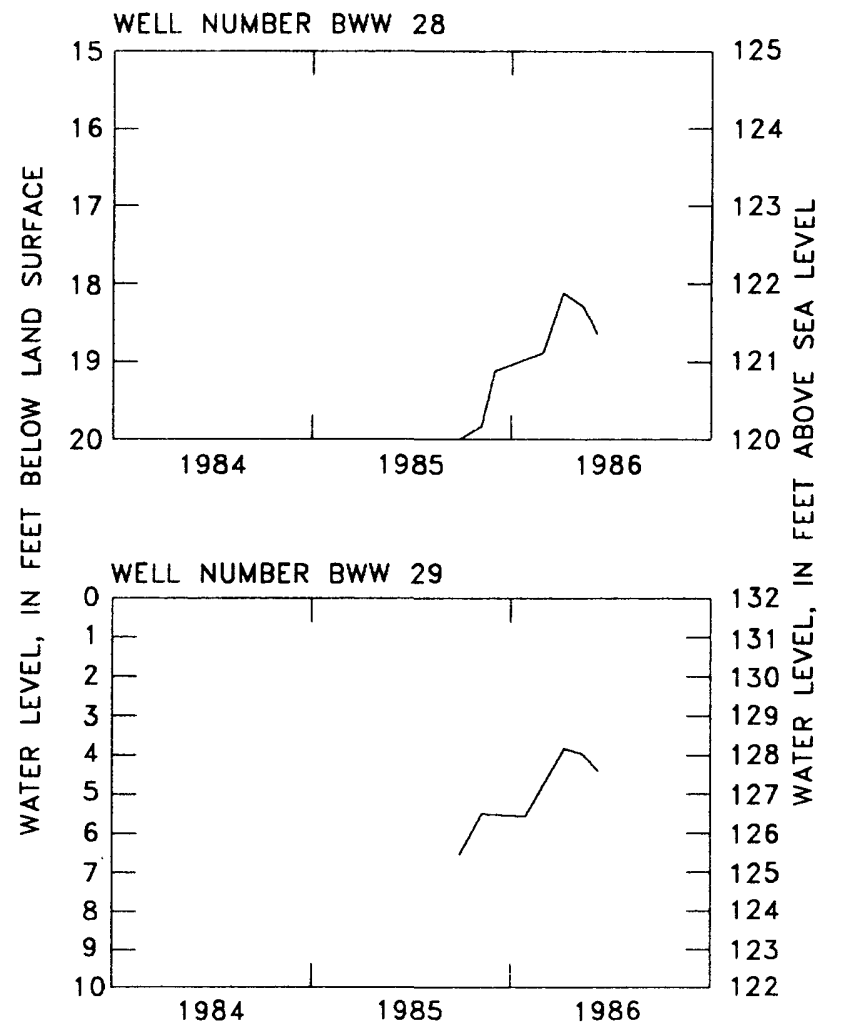

Figure 4.-Periodic water levels at wel number BWW 28 and BWW 29.

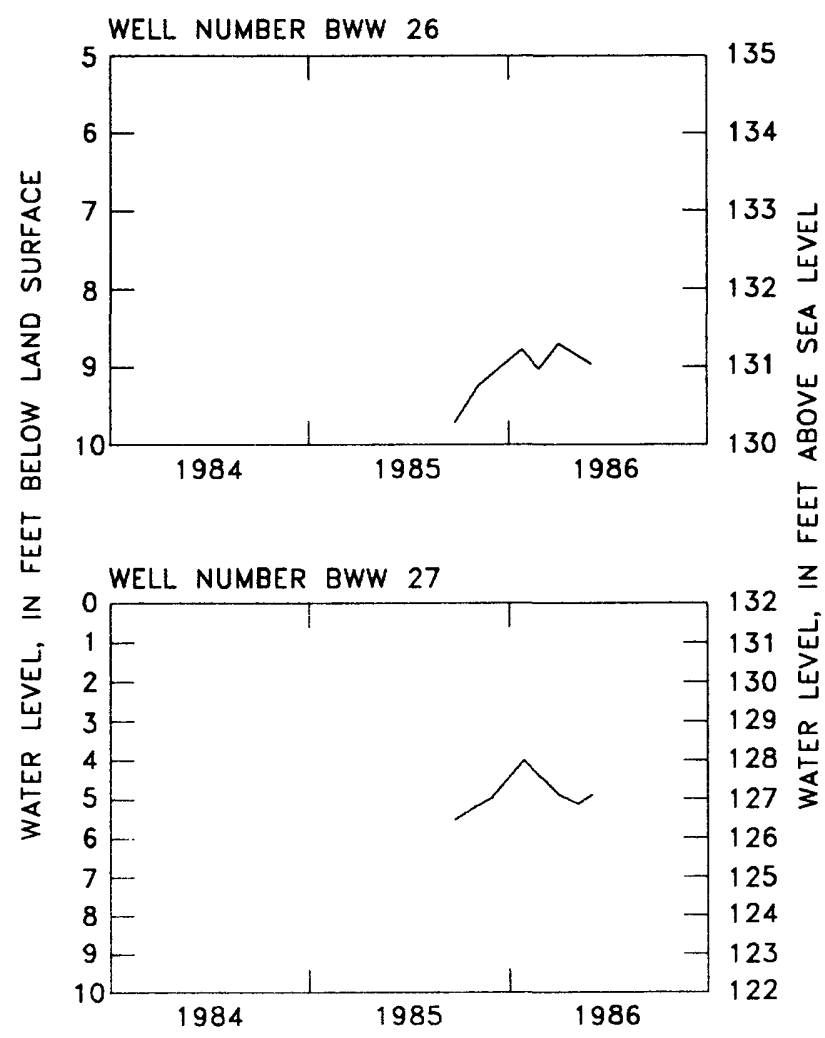

Figure 3.--Periodic water levels at well number BWW 26 and BWW 27.

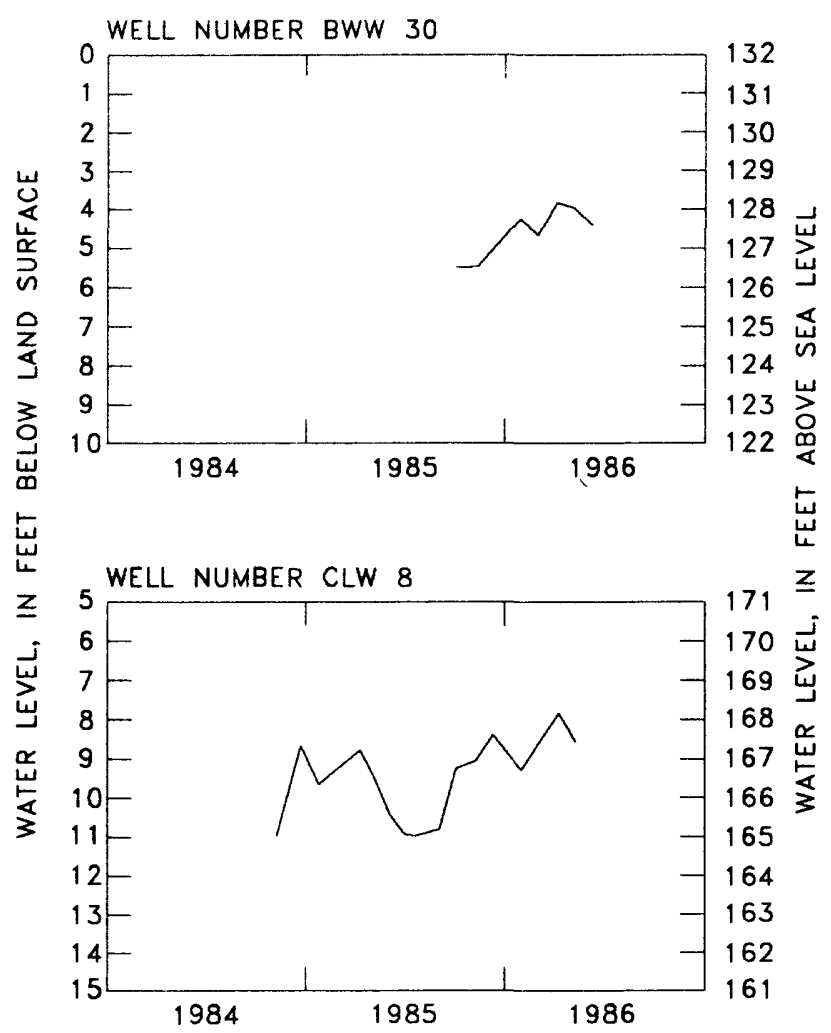

Figure 5.-Periodic water levels at well number BWW 30 and CLW 8. 


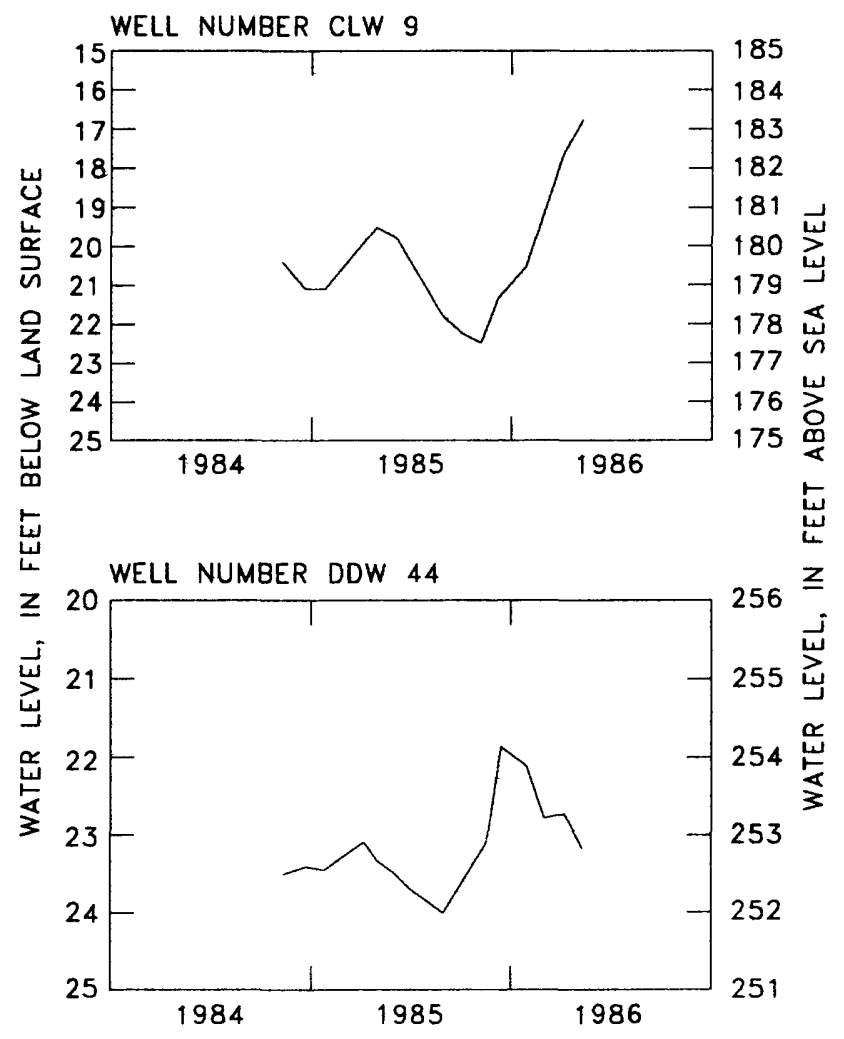

Figure 6.-Periodic water levels at well number CLW 9 and DDW 44.

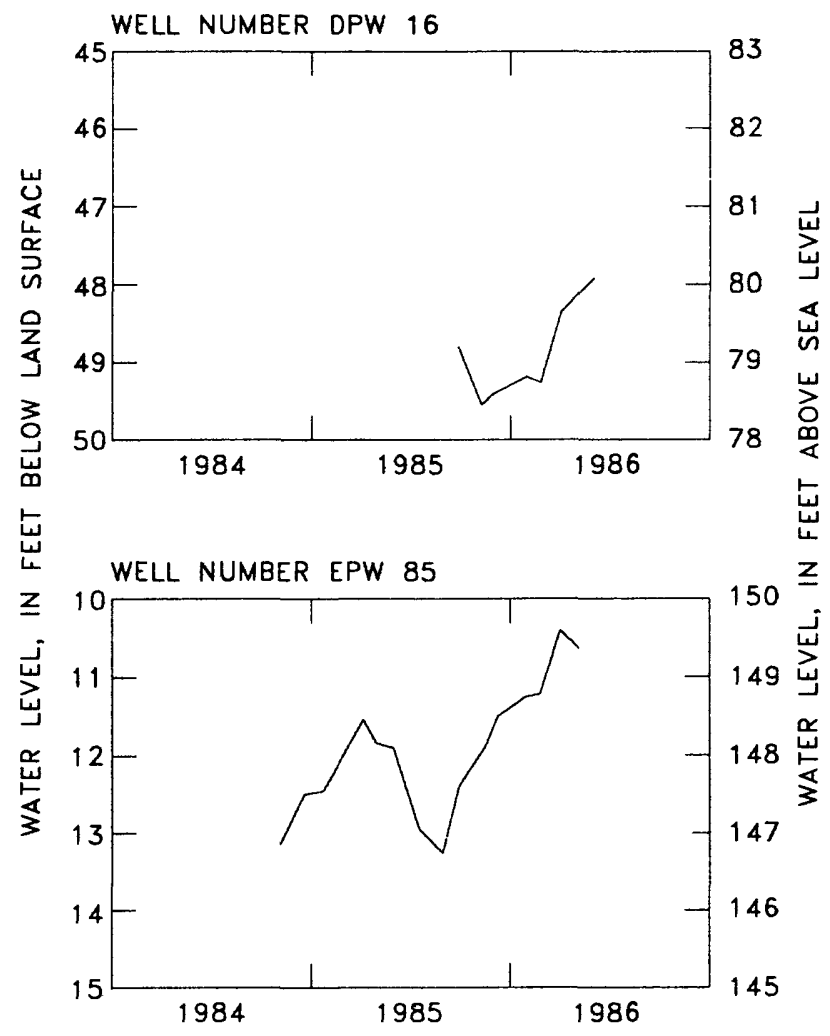

Figure 8.-Periodic water levels at well number DPW 16 and EPW 85.

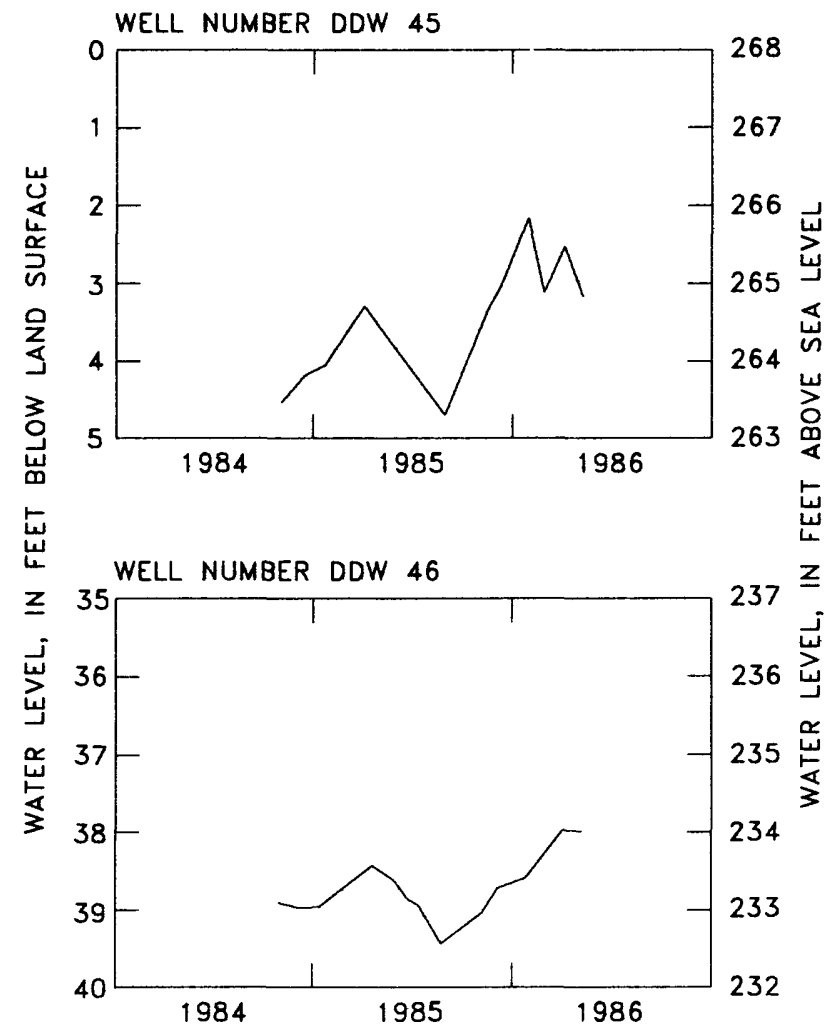

Figure 7.-Periodic water levels at DDW 45 and DDW 46.

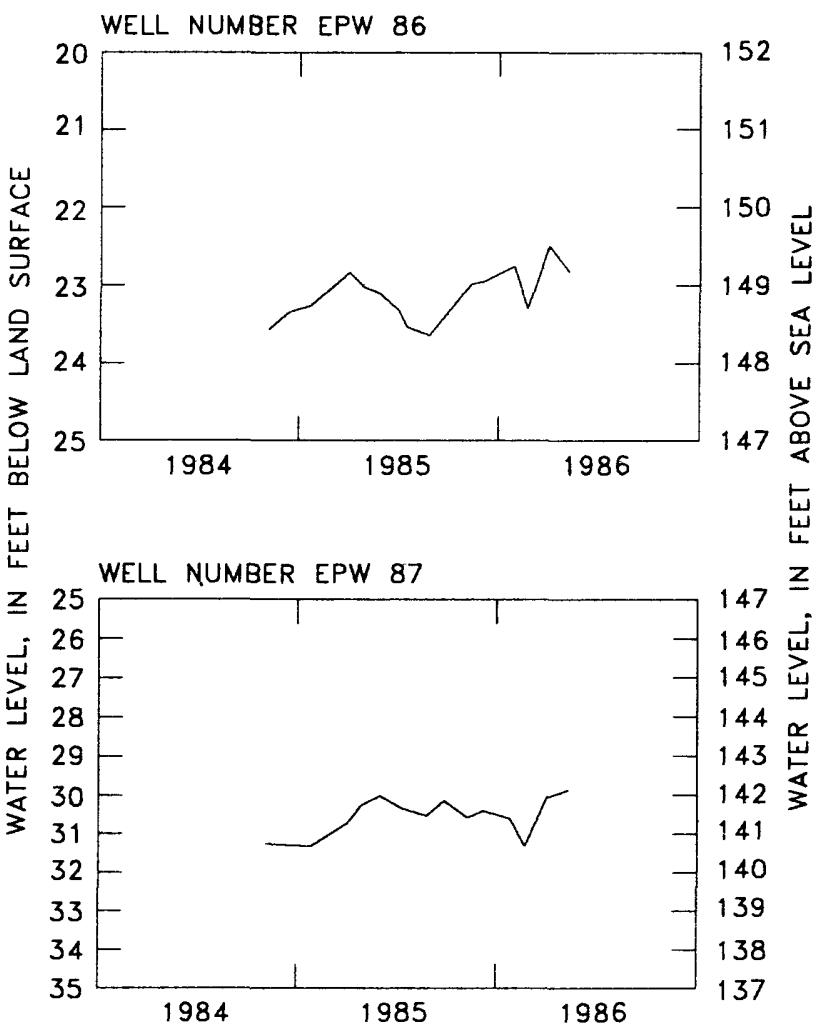

Figure 9.-Periodic water levels at well number EPW 86 and EPW 87. 


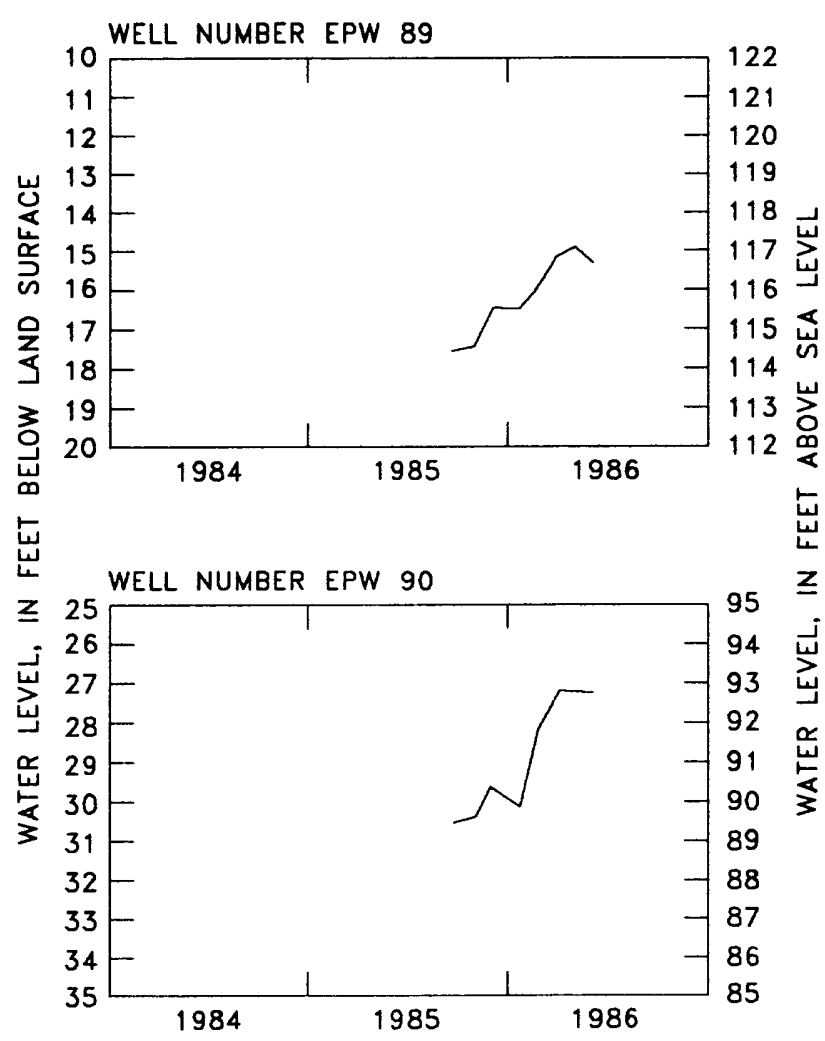

Figure 10.--Periodic water levels at well number EPW 89 and EPW 90.

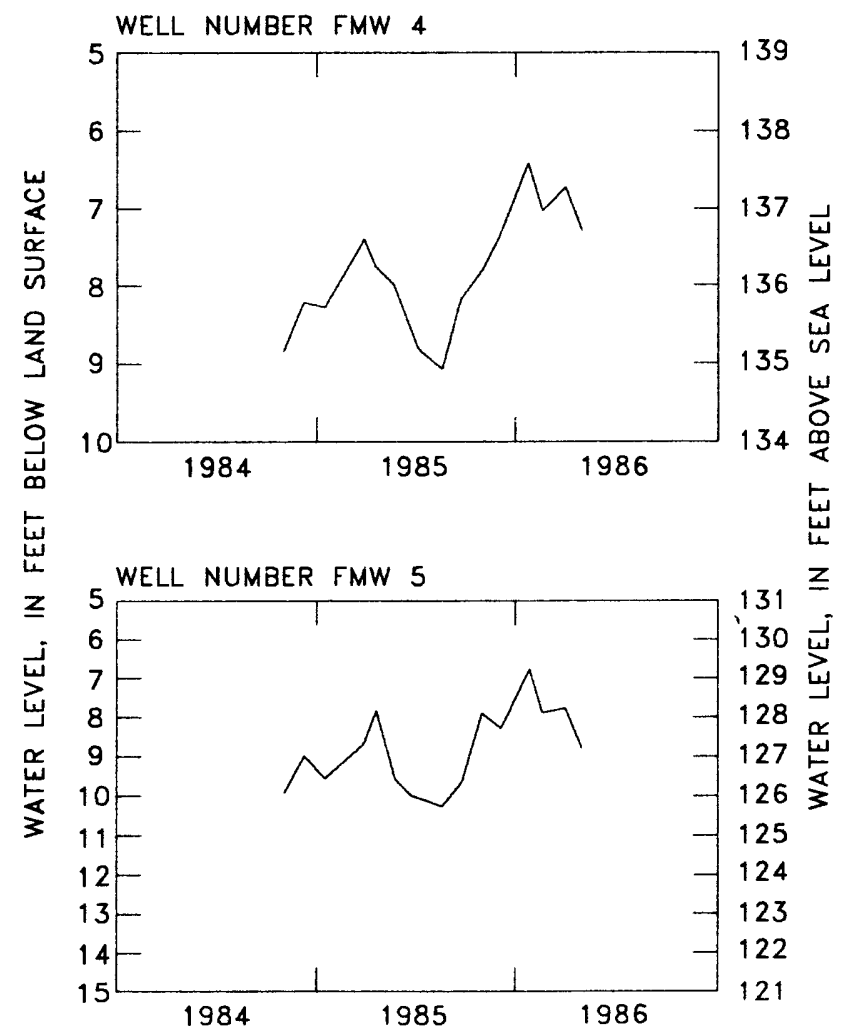

Figure 12.--Periodic water levels al well number FMW 4 and FMW 5.

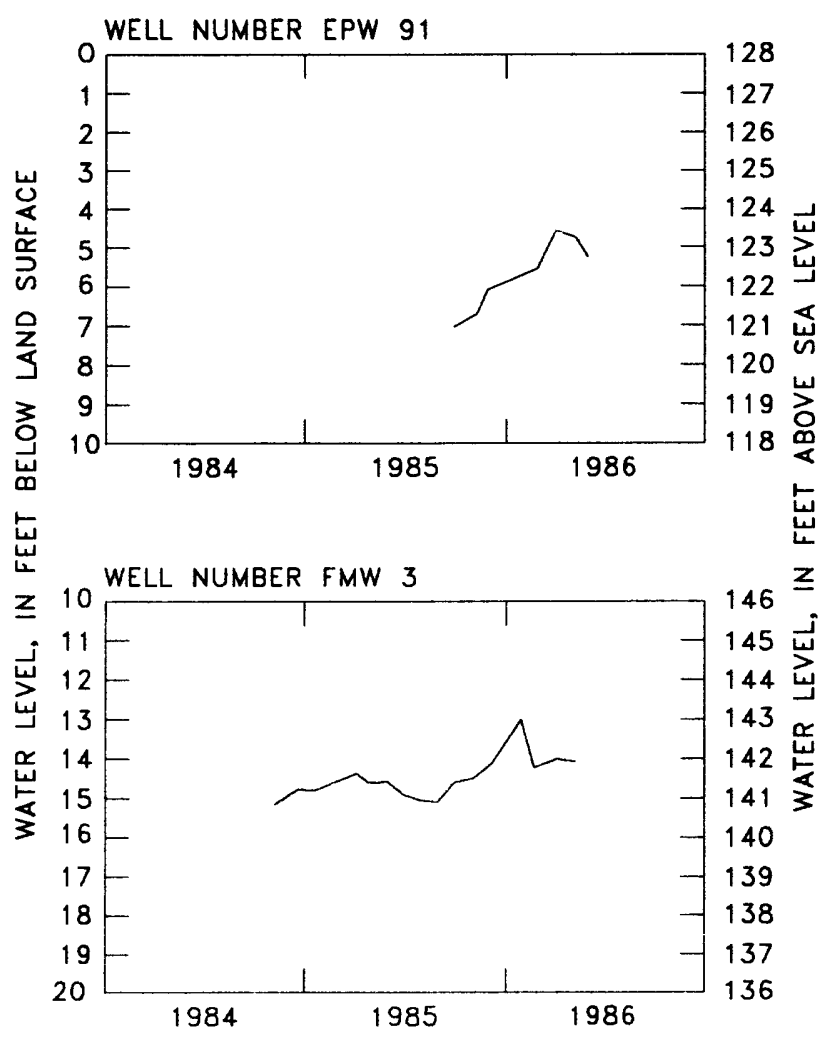

Figure 11.--Periodic water levels at well number EPW 91 and FMW 3.

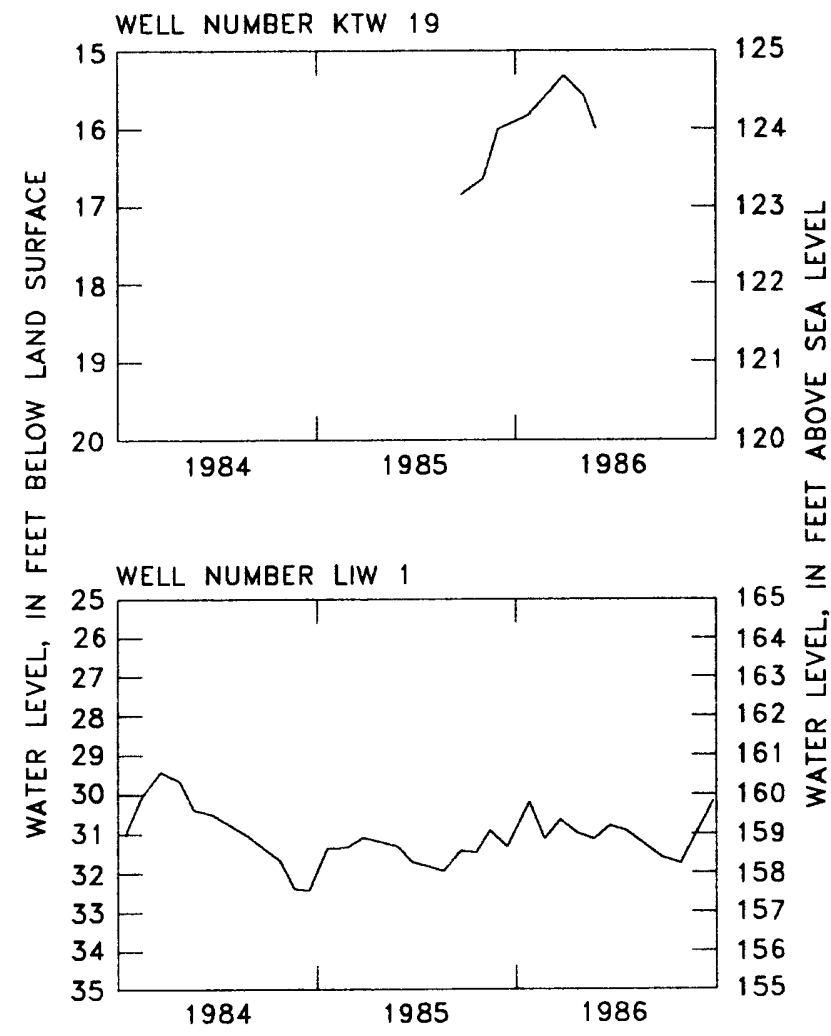

Figure 13.-Periodic water levels at well number KTW 19 and LIW 1. 


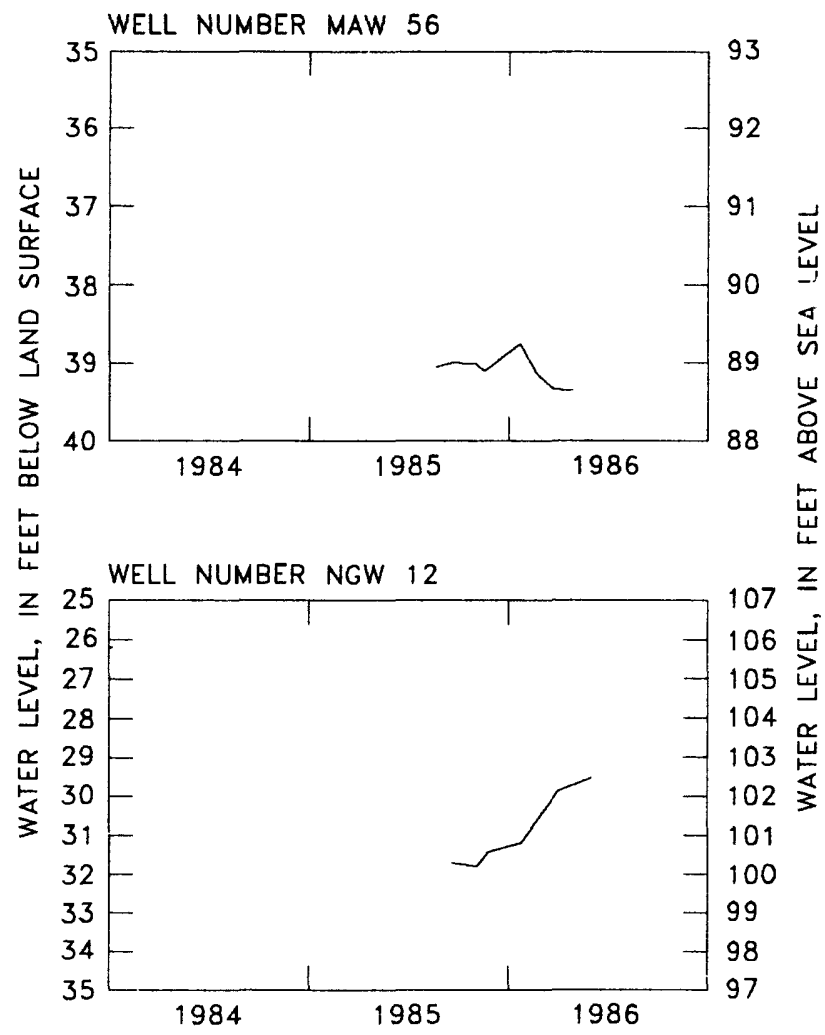

Figure 14.--Periodic water levels at well number MAW 56 and NGW 12.

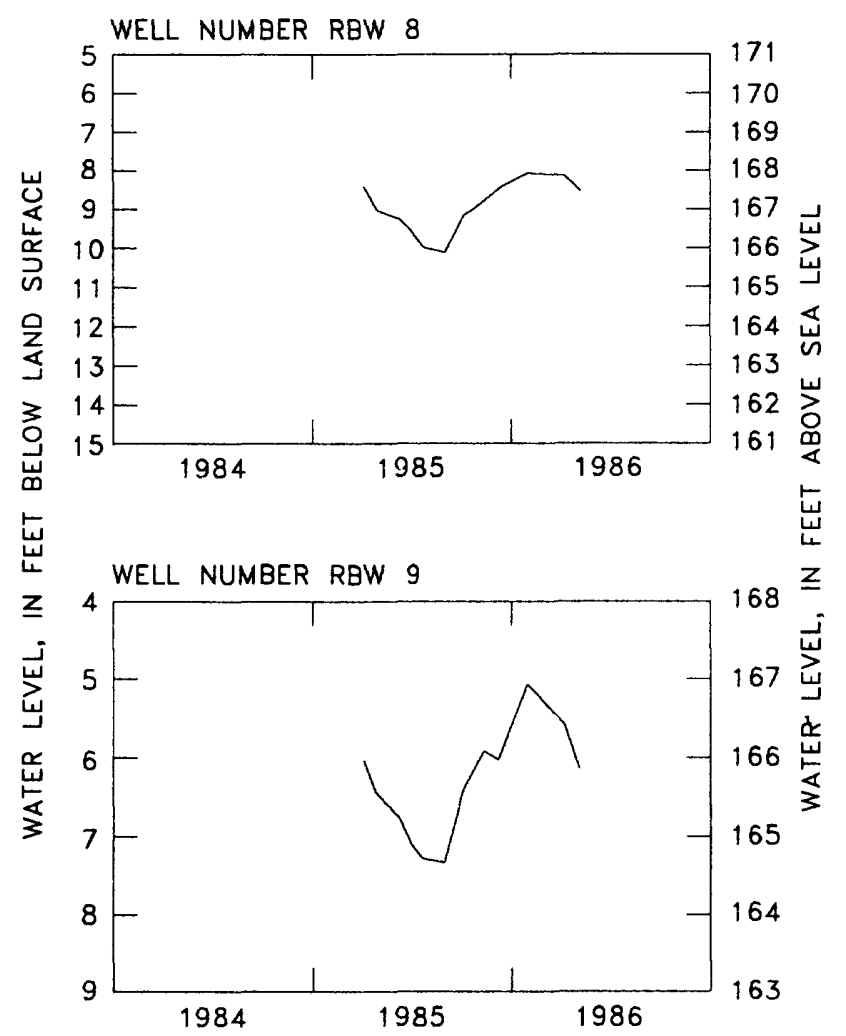

Figure 16.-Periodic water levels at well number RBW 8 and RBW 9.

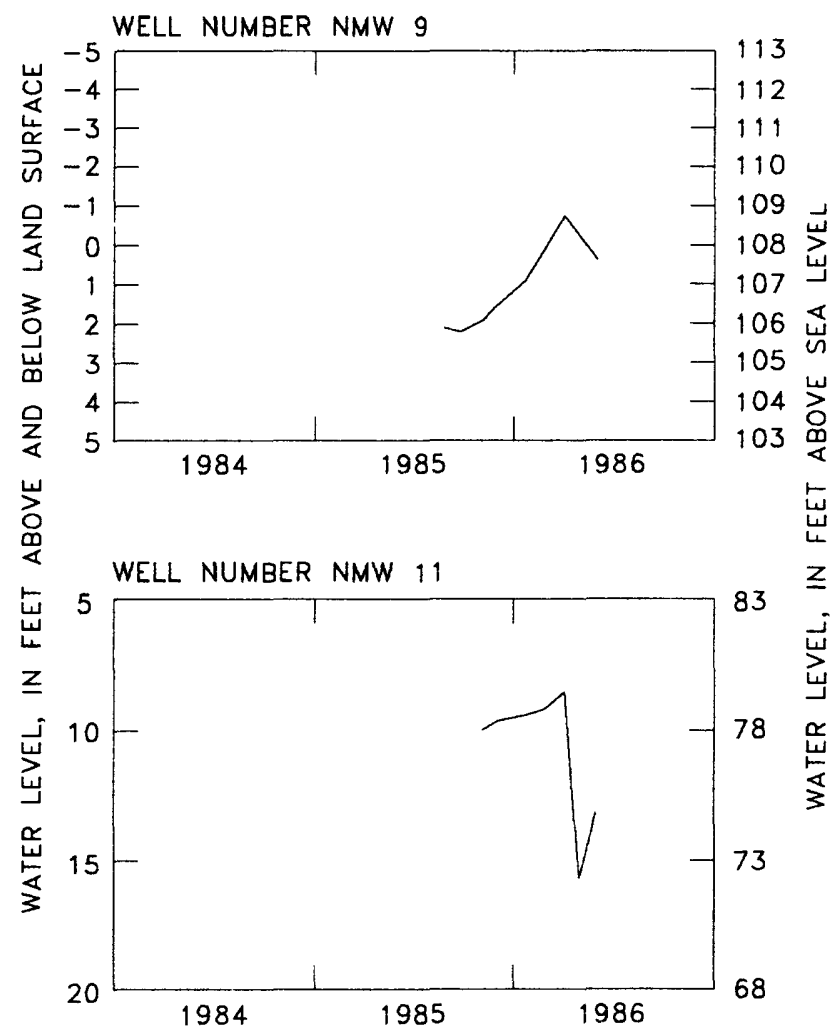

Figure 15.-Periodic water levels at well number NMW 9 and NMW 11.

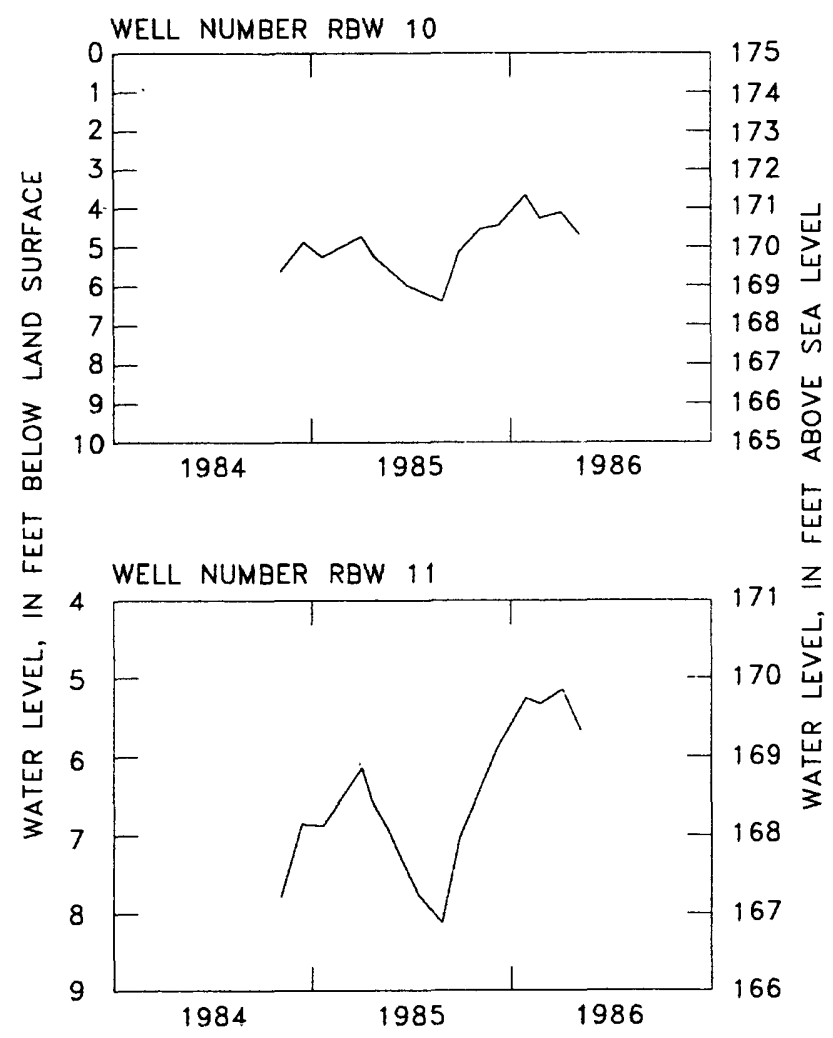

Figure 17.-Periodic water levels at well number RBW 10 and RBW 11. 


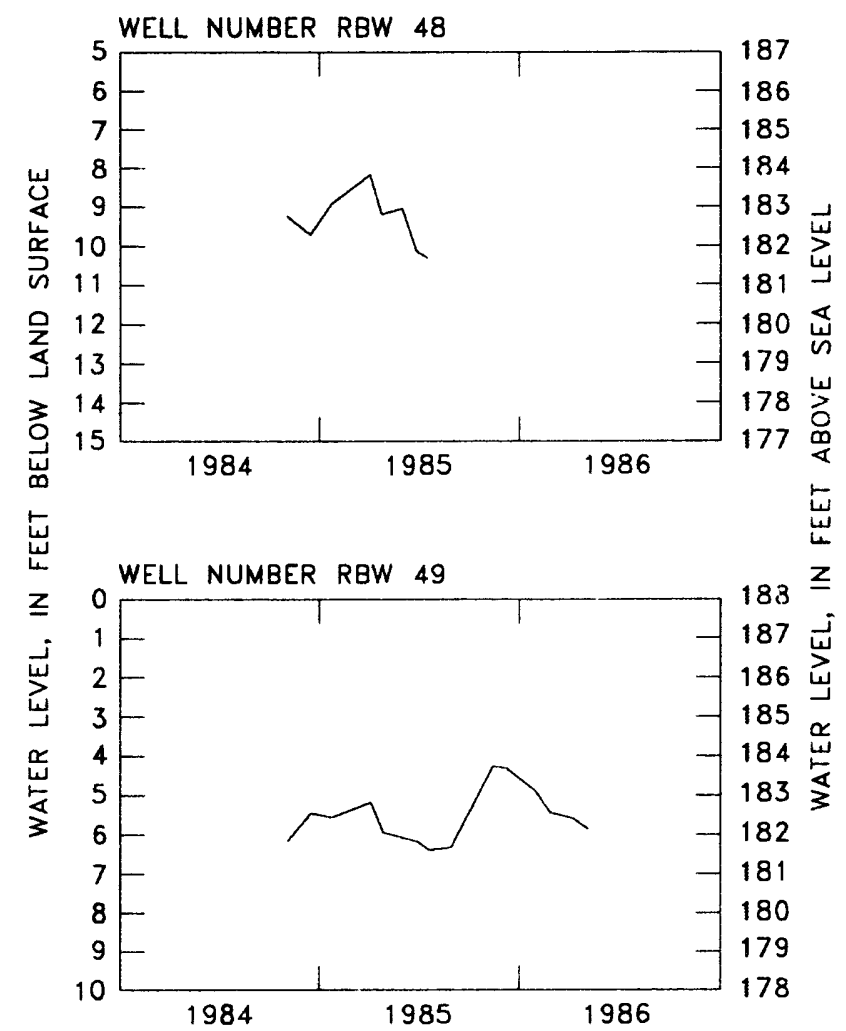

Figure 18.-Periodic water levels at well number RBW 48 and RBW 49.

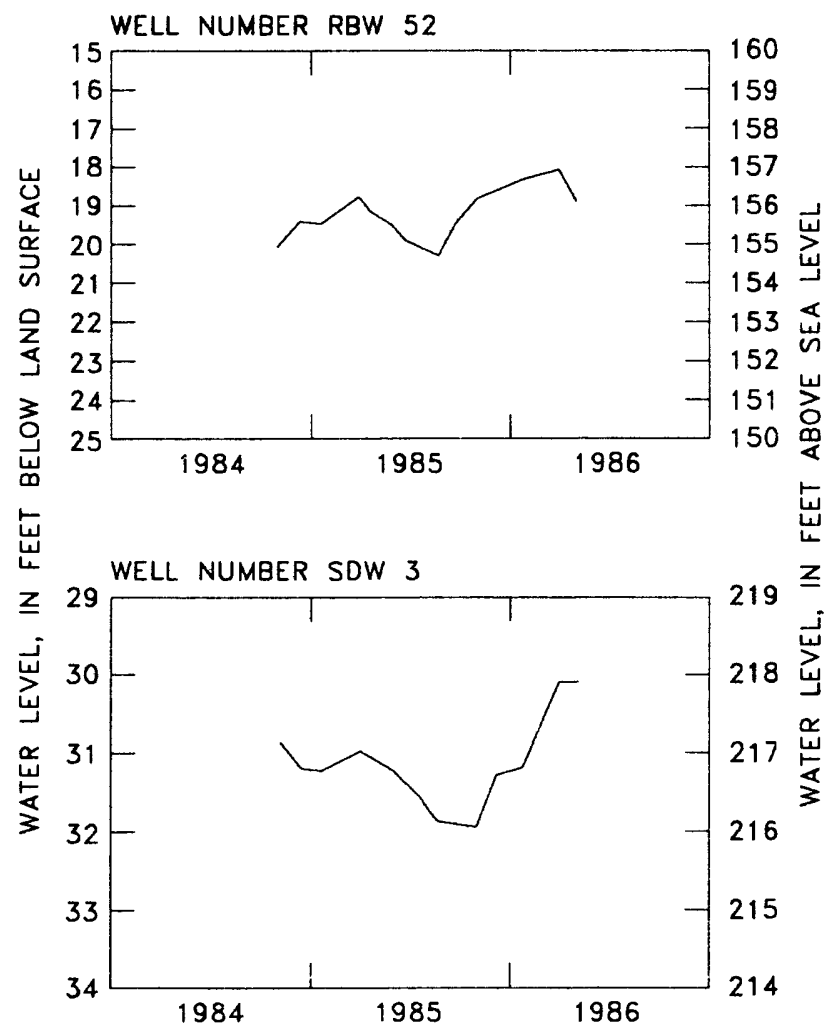

Figure 20.-Periodic water levels at well number RBW 52 and SDW 3.

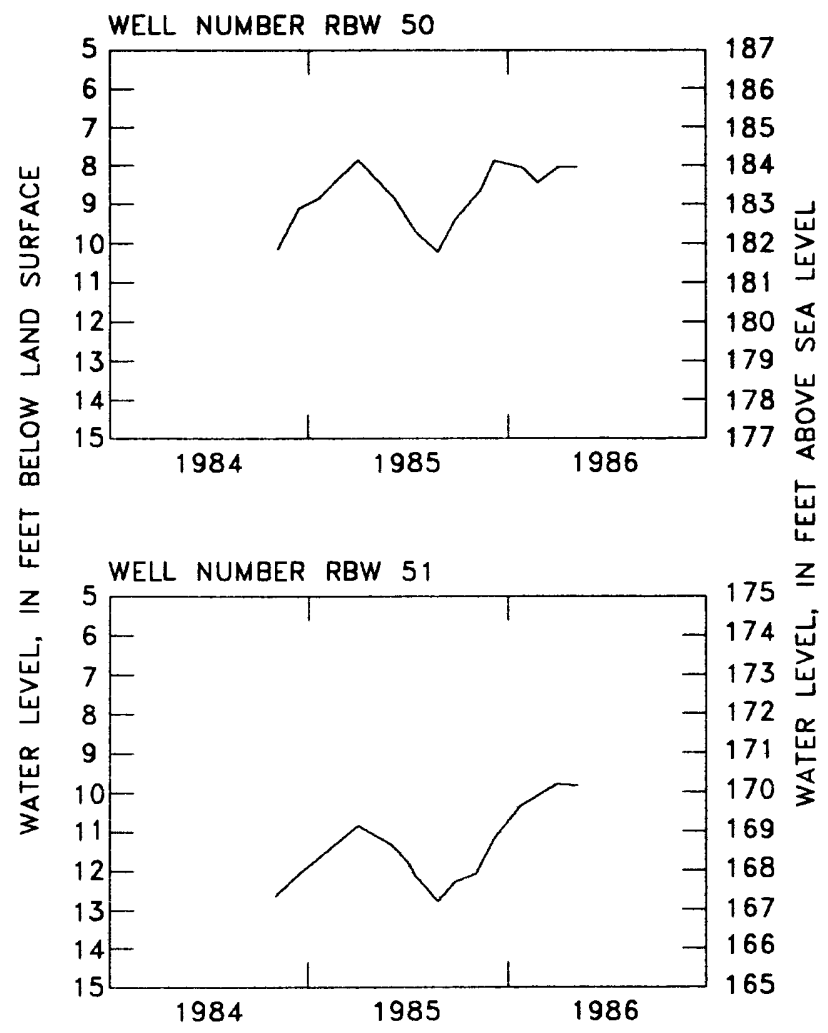

Figure 19.--Periodic water levels at well number RBW 50 and RBW 51.

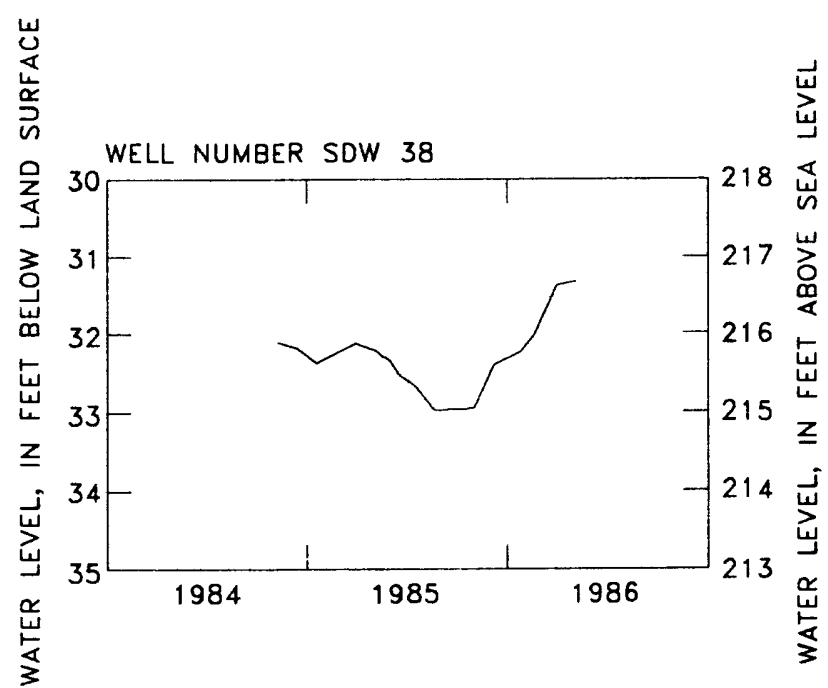

Figure 21.-Periodic water levels at well number SDW38. 


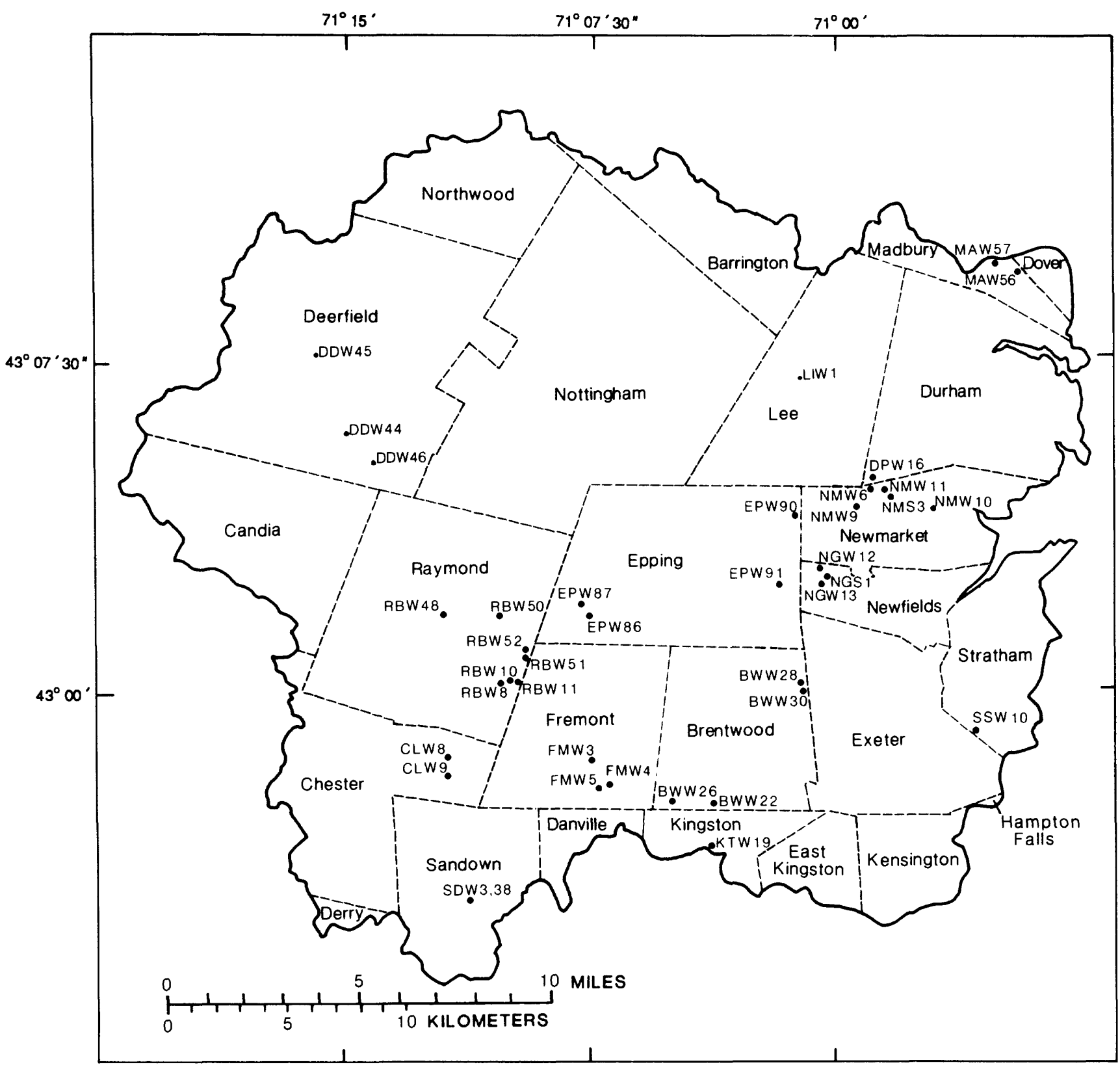

\section{EXPLANATION}

- BOUNDARY OF STUDY AREA

-.-- TOWN BOUNDARY

- RBW48 WATER - QUALITY SAMPLING LOCATION

A WELL OR SPRING WITH LOCAL IDENTIFIER

Figure 22.--Ground-water-quality sampling locations. 


\section{Table 2.--Description of wells, borings and springs}

Local site number: First two characters indicate U.S. Geological Survey town code. Third character indicates--A, borings done for hydrologic purposes; B, borings done primarily for constructional purposes; S, spring; W, well.

Latitude, Longitude: Accurate within 5 seconds.

Owner or user: NHDOT, New Hampshire Department of Transportation; USCOE, United States Army Corps of Engineers; BLDRS, Builders.

Altitude: Altitude is expressed in feet above sea level. Most of the time is estimated from U.S. Geological Survey topographic maps, accurate to \pm 5 or 10 feet (half the contour interval). Some altitudes were determined by use of levels and are accurate to \pm 0.1 foot as shown. Altitudes of U.S. Geological Survey wells and test holes drilled for this study were determined by altimeter and are accurate to \pm 4 feet.

Diameter: Diameters of the well easings in inches.

Depth to Bottom of Casing: Depth to the bottom of well casing in feet below land-surface datum.

Casing material: Materials from which the casing are made: B, Brick; C, Concrete; G, Galvanized iron; I, Wrought iron; P, PVC or other plastic; R, Rock or stone; S, steel; or T, tile.

Type of Finish: Method of finish or nature of the openings that allow water to enter the well: G, Gravel-pack well with a screen; $O$, Open end (cased to the bottom of the well, so water can only enter the well only through the bottom of the hole); $P$, Perforated or slotted with holes punched or slots cut into the casing to admit water; S, Screen (manufactured); T, Sand point for driven wells; X, Open hole where the casing does not extend to the bottom of the well (usually a bedrock well).

Depth to Bottom of Open Section: Depth to the bottom of the screen or open section in which water enters the well in feet below land-surface datum.

Type of site: Brw, Bedrock well; TH, Test hole; Sp, Spring.

Wells or borings in Surficial Deposits: Bor, Bored or augered; Dug, dug well; Dvn, driven well; Cbl, Cable-tool well; Wsh, Drive and wash well.

Water level: In feet below land-surface datum; negative sign indicates water level above land surface datum; mm-dd-yy, month-day-year.

Use:

Use of site: 0, Observation well drilled for water-level or water-quality observations; T, Test hole.

Use of water: C, commercial; F, Fire; H, Domestic; I, Irrigation; J, Industrial (Cooling); M, Medical;

N, Industrial; P, Public Supply; R, Recreation; S, Stock; U, Unused; IN, Institutional.

Maximum Yield: Discharge in gallons per minute.

Drawdown: In feet, observed at a pumping well.

Specific Capacity: In gallons per minute per foot of drawdown.

Pumping Period: The length of time, in hours, that the well was pumped prior to the measurement of production levels.

Remarks: CA, chemical analysis summarized in table 4; USGS, well or test hole drilled by the U.S.

Geological Survey for this investigation; WSPCC, New Hampshire Water Supply and Pollution Control Commission. 


\section{Table 2.--Description of wells,}

[ft, feet; in., inch; gal, gallon; min, minute;

\begin{tabular}{|c|c|c|c|c|c|c|c|c|c|c|c|}
\hline $\begin{array}{l}\text { Local } \\
\text { site } \\
\text { number }\end{array}$ & $\begin{array}{l}\text { Lati- } \\
\text { tude }\end{array}$ & $\begin{array}{l}\text { Long- } \\
\text { tude }\end{array}$ & Owner or user & $\begin{array}{l}\text { Year } \\
\text { comp- } \\
\text { leted }\end{array}$ & $\begin{array}{l}\text { Altitude } \\
\text { above } \\
\text { sea } \\
\text { level } \\
\text { (ft) }\end{array}$ & $\begin{array}{l}\text { Diam- } \\
\text { eter } \\
\text { of } \\
\text { well } \\
\text { (in.) }\end{array}$ & $\begin{array}{l}\text { Depth to } \\
\text { bottom } \\
\text { of } \\
\text { casing } \\
\text { (ft) }\end{array}$ & $\begin{array}{l}\text { Casing } \\
\text { mate- } \\
\text { rial }\end{array}$ & $\begin{array}{l} \\
\text { Type } \\
\text { of } \\
\text { fin- } \\
\text { ish }\end{array}$ & $\begin{array}{l}\text { Depth to } \\
\text { bottom } \\
\text { of } \\
\text { open } \\
\text { section } \\
\text { (ft) }\end{array}$ & $\begin{array}{l}\text { Type } \\
\text { of } \\
\text { site }\end{array}$ \\
\hline
\end{tabular}

ROCKINGHAM COUNTY

\begin{tabular}{|c|c|c|c|c|c|c|c|c|c|c|c|c|}
\hline \multicolumn{13}{|c|}{ Brentwood } \\
\hline BWA & 1 & 425914 & 710245 & -- & 1985 & 120 & -- & -- & -- & -- & -- & Bor \\
\hline BWA & 2 & 425843 & 710224 & -- & 1985 & 110 & -- & -- & -- & -- & -- & Bor \\
\hline BWA & 3 & 425838 & 710249 & -- & 1985 & 130 & -- & -- & - & -- & -- & Bor \\
\hline BWA & 4 & 425935 & 710107 & -- & 1985 & 125 & -- & -- & -- & -- & -- & Bor \\
\hline BWA & 16 & 425927 & 710154 & -- & 1956 & 100 & -- & -- & -- & -- & -- & Bor \\
\hline BWA & 17 & 425846 & 710417 & -- & 1956 & 110 & -- & -- & -- & -- & -- & Bor \\
\hline BWB & 1 & 425741 & 710228 & NHDOT & 1958 & 69.2 & -- & -- & -- & -- & -- & Wsh \\
\hline BWB & 2 & 425839 & 710426 & NHDOT & 1958 & 104.0 & -- & -- & -- & -- & -- & Wsh \\
\hline BWS & 1 & 430032 & 710306 & ROCKINGHAM CO & -- & 125 & -- & -- & -- & -- & -- & $\mathrm{Sp}$ \\
\hline BWW & 1 & 430043 & 710307 & ROIKINGHAM CO & 1948 & 140 & 10 & -- & $\mathrm{s}$ & -- & -- & $\mathrm{Cb} 1$ \\
\hline BWW & 2 & 425928 & 710138 & SPAULDING ALFRED & -- & 105 & 36 & -- & B & -- & -- & Dug \\
\hline BWW & 3 & 425908 & 710222 & PIERCE JOHN B & -- & 95 & 48 & -- & $\mathrm{R}$ & -- & -- & Dug \\
\hline BWW & 4 & 425906 & 710352 & PARKER C A & -- & 145 & 36 & -- & B & -- & -- & Dug \\
\hline BWW & 5 & 425734 & 710109 & COE HARRY & -- & 210 & 36 & -- & $\mathrm{R}$ & -- & -- & Dug \\
\hline BWW & 6 & 425737 & 710122 & STEVENS JOHN W & 1949 & 210 & 6 & -- & $\mathbf{S}$ & -- & -- & BrW \\
\hline BWW & 7 & 425745 & 710327 & NH ST FISH \& GAME & 1950 & 135 & 8 & -- & S & -- & -- & $\mathrm{BrW}$ \\
\hline BWW & 8 & 425925 & 710139 & SPAULDING ALFRED & 1952 & 95 & 48 & -- & $\mathrm{C}$ & -- & -- & Dug \\
\hline BWW & 9 & 425826 & 710015 & SWASEY JOHN & -- & 120 & 36 & -- & B & -- & -- & Dug \\
\hline BWW & 10 & 425835 & 710016 & GALLAGHER GRANT A & 1954 & 150 & 8 & -- & s & -- & -- & $\mathrm{BrW}$ \\
\hline BWW & 11 & 430040 & 710345 & SPAULDING $\mathrm{K} C$ & -- & 155 & 40 & -- & $\mathrm{B}$ & -- & -- & Dug \\
\hline BWW & 12 & 430039 & 710210 & THYNG'S FARM & 1945 & 155 & 6 & -- & $\mathbf{S}$ & -- & -- & $\mathrm{BrW}$ \\
\hline BWW & 13 & 430003 & 710135 & SANBORN GEORGE O & 1915 & 145 & 36 & -- & $\mathrm{B}$ & -- & -- & Dug \\
\hline BWW & 14 & 425830 & 710057 & SOROKIN M & 1951 & 130 & 6 & 90.0 & S & -- & -- & $\mathrm{BrW}$ \\
\hline BWW & 15 & 425744 & 710446 & BARTLETT WILLIAM & 1935 & 140 & 24 & -- & $\mathrm{T}$ & -- & -- & Dug \\
\hline BWW & 19 & 430048 & 710316 & ROCKINGHAM & 1972 & 130 & -- & -- & -- & S & -- & - \\
\hline BWW & 20 & 430106 & 710149 & NEW ENGLAND DRAGWAY & 1977 & 110 & -- & -- & -- & -- & -- & -- \\
\hline BWW & 21 & 425740 & 710313 & -- & 1985 & 136 & 2 & 19.5 & $\mathrm{P}$ & $\mathrm{P}$ & 22.0 & Bor \\
\hline BWW & 22 & 425742 & 710332 & -- & 1985 & 144 & 2 & 47.5 & $\mathrm{P}$ & $P$ & 50.0 & Bor \\
\hline BWW & 26 & 425738 & 710441 & -- & 1985 & 140 & 2 & 52.5 & $\mathrm{P}$ & $\mathrm{P}$ & 55.0 & Bor \\
\hline BWW & 27 & 430049 & 710201 & -- & 1985 & 132 & 2 & 9.5 & $\mathrm{P}$ & $P$ & 12.0 & Bor \\
\hline BWW & 28 & 430023 & 710044 & -- & 1985 & 140 & 2 & 29.5 & $\mathrm{P}$ & 0 & 32.0 & Bor \\
\hline BWW & 29 & 430015 & 710042 & - & 1985 & 132 & 2 & 27.5 & $\mathrm{P}$ & $\mathrm{P}$ & 30.0 & Bor \\
\hline BWW & 30 & 430015 & 710042 & -- & 1985 & 132 & 2 & 37.5 & $\mathrm{P}$ & $\mathrm{P}$ & 40.0 & Bor \\
\hline BWW & 31 & 430021 & 710106 & EXETER, TOWN OF & 1985 & 120 & -- & -- & -- & -- & -- & -- \\
\hline BWW & 32 & 430017 & 710106 & BRENTWOOD, TOWN OF & 1984 & 120 & -- & -- & -- & -- & -- & -- \\
\hline BWW & 33 & 430026 & 710106 & -- & 1985 & 130 & 3 & 60.0 & -- & -- & 65.0 & -- \\
\hline BWW & 34 & 430026 & 710100 & EXETER, TOWN OF & 1985 & 120 & -- & -- & -- & $\mathrm{S}$ & -- & -- \\
\hline BWW & 35 & 430027 & 710100 & EXETER, TOWN OF & 1984 & 120 & -- & -- & -- & $\mathrm{S}$ & -- & -- \\
\hline BWW & 36 & 430029 & 710100 & BRENTWOOD, TOWN OF & 1985 & 120 & -- & -- & -- & $\mathbf{S}$ & -- & -- \\
\hline BWW & 37 & 430028 & 710104 & BRENTWOOD, TOWN OF & 1985 & 120 & -- & -- & -- & $s$ & -- & -- \\
\hline BWW & 38 & 430027 & 710107 & EXETER, TOWN OF & 1984 & 120 & -- & -- & -- & $\mathrm{S}$ & -- & -- \\
\hline BWW & 39 & 430026 & 710111 & BRENTWOOD, TOWN OF & 1985 & 120 & -- & -- & -- & $\mathbf{s}$ & -- & -- \\
\hline BWW & 41 & 430029 & 710119 & EXETER, TOWN OF & 1984 & 120 & -- & -- & -- & $\mathrm{S}$ & -- & -- \\
\hline
\end{tabular}




\section{borings, and springs}

hr, hour; --, no data available]

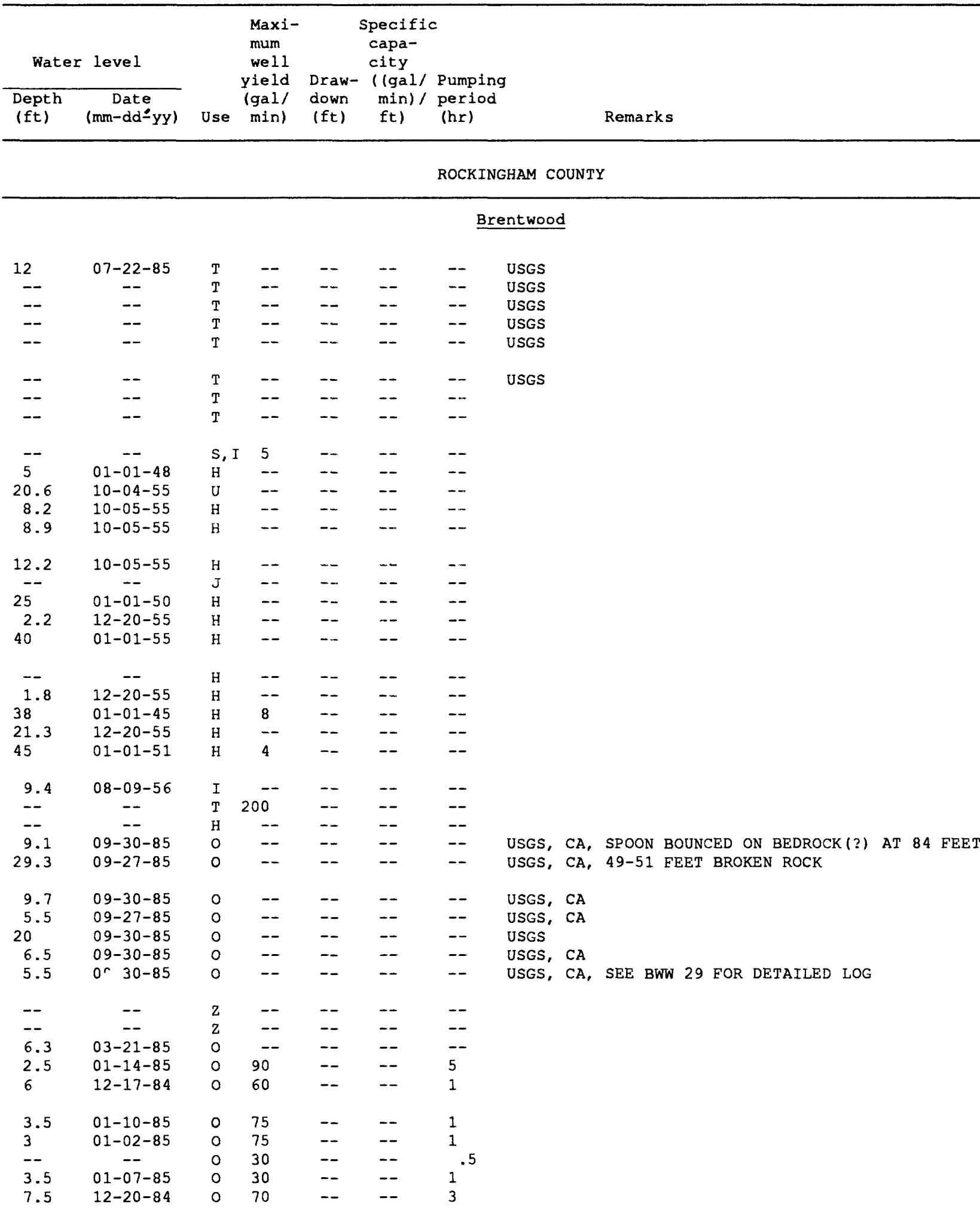




\begin{tabular}{|c|c|c|c|c|c|c|c|c|c|c|c|}
\hline $\begin{array}{l}\text { Local } \\
\text { site } \\
\text { number }\end{array}$ & $\begin{array}{l}\text { Lati- } \\
\text { tude }\end{array}$ & $\begin{array}{l}\text { Long- } \\
\text { tude }\end{array}$ & Owner or user & $\begin{array}{l}\text { Year } \\
\text { comp- } \\
\text { leted }\end{array}$ & $\begin{array}{l}\text { Altitude } \\
\text { above } \\
\text { sea } \\
\text { level } \\
\text { (ft) }\end{array}$ & $\begin{array}{l}\text { Diam- } \\
\text { eter } \\
\text { of } \\
\text { well } \\
\text { (in.) }\end{array}$ & $\begin{array}{l}\text { Depth to } \\
\text { bottom } \\
\text { of } \\
\text { casing } \\
\text { (ft) }\end{array}$ & $\begin{array}{l}\text { Casing } \\
\text { mate- } \\
\text { rial }\end{array}$ & $\begin{array}{l}\text { Type } \\
\text { of } \\
\text { fin- } \\
\text { ish }\end{array}$ & $\begin{array}{l}\text { Depth to } \\
\text { bottom } \\
\text { of } \\
\text { open } \\
\text { section } \\
\text { (ft) }\end{array}$ & $\begin{array}{l}\text { Type } \\
\text { of } \\
\text { site }\end{array}$ \\
\hline
\end{tabular}

Brentwood--Continued

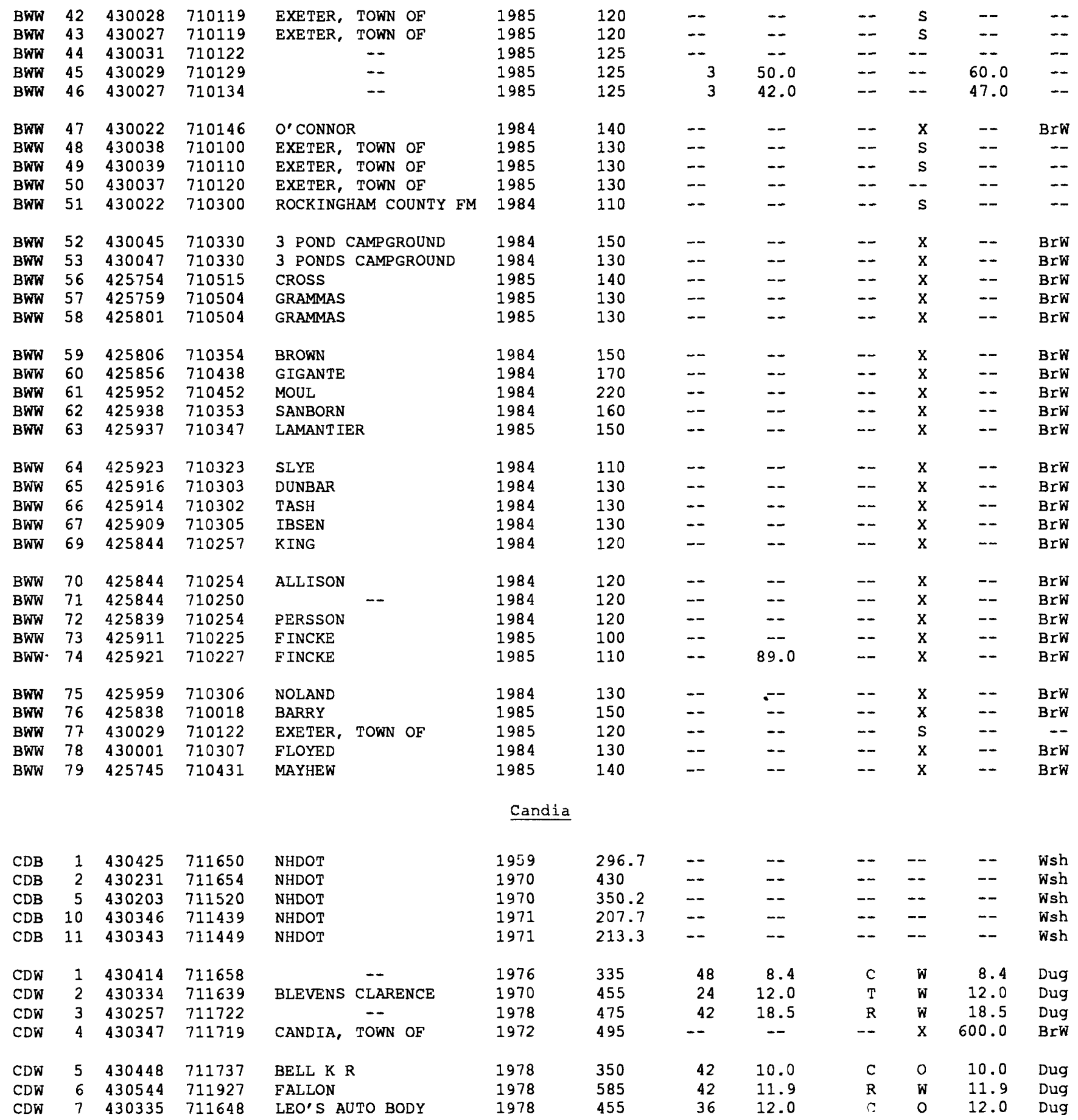




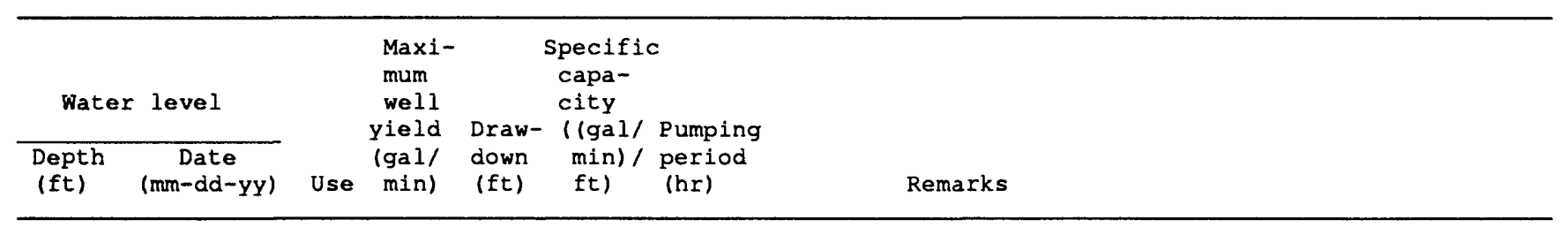

\section{Brentwood--Cont inued}

\begin{tabular}{|c|c|c|c|c|c|c|}
\hline $\begin{array}{l}7.4 \\
6.4\end{array}$ & $\begin{array}{l}01-04-85 \\
12-26-85\end{array}$ & $\begin{array}{l}0 \\
0\end{array}$ & 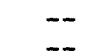 & - & -- & -- \\
\hline-- & - & 0 & - & -- & - & -- \\
\hline 10.8 & $03-18-85$ & 0 & 50 & -- & -- & -- \\
\hline 11.1 & $03-19-85$ & 0 & -- & - & - & -- \\
\hline-- & -- & $\mathrm{H}$ & 4 & -- & - & -- \\
\hline-- & - & 0 & -- & - & -- & $-\sim$ \\
\hline- & - & 0 & - & - & - & -- \\
\hline- & - & $\mathrm{z}$ & -- & -- & - & -- \\
\hline 4.3 & $09-17-84$ & -- & 201 & -- & -- & 144 \\
\hline 12 & $11-02-84$ & P & 10 & -- & -- & 32 \\
\hline 14 & $11-02-84$ & P & 8 & -- & -- & 32 \\
\hline- & -- & $\mathrm{H}$ & 12 & - & -- & -- \\
\hline-- & -- & $\mathrm{H}$ & 3 & -- & -- & 1 \\
\hline$\sim$ & - & $\mathrm{H}$ & 4 & - & $\cdots$ & 1 \\
\hline-- & -- & $\mathrm{H}$ & 8.5 & -- & -- & .5 \\
\hline 10 & $08-30-84$ & C & 2.5 & $\sim$ & - & 1 \\
\hline-- & - & $\mathrm{H}$ & 5 & -- & -- & 1 \\
\hline 20 & $10-23-84$ & $\mathrm{H}$ & 5 & - & -- & 1 \\
\hline-- & - & $\mathrm{H}$ & 1.5 & $-\infty$ & -- & 2 \\
\hline 5 & $09-22-84$ & $\mathrm{H}$ & 4 & -- & -- & 1 \\
\hline$\sim$ & $-\infty$ & $\mathrm{H}$ & 100 & -- & $\sim$ & .5 \\
\hline 8 & $09-27-84$ & $\mathrm{H}$ & 30 & -- & - & 1 \\
\hline- & - & $\mathrm{H}$ & 12 & -- & -- & 1 \\
\hline- & -- & -- & 20 & $-\infty$ & -- & -- \\
\hline-- & -- & $\mathrm{H}$ & 30 & - & -- & 1 \\
\hline$\cdots$ & $\sim$ & -- & 4 & - & - & -- \\
\hline- & - & $\mathrm{H}$ & 8 & -- & -- & - \\
\hline-- & -- & $\mathrm{H}$ & 20 & -- & -- & -- \\
\hline-- & -- & $\mathrm{H}$ & 25 & -- & -- & -- \\
\hline-- & -- & $\mathrm{H}$ & 20 & -- & -- & 1 \\
\hline-- & -- & $\mathrm{H}$ & 50 & - & -- & -- \\
\hline 9.4 & $01-03-85$ & 0 & 60 & -- & -- & 1 \\
\hline 10 & $07-26-84$ & $\mathrm{H}$ & 30 & -- & - & 1 \\
\hline 20 & $03-19-85$ & $\mathrm{H}$ & 5 & -- & -- & 4 \\
\hline
\end{tabular}

Candia

$\begin{array}{lllllll}-- & -- & T & -- & -- & -- & -- \\ -- & -- & T & -- & -- & -- & - \\ -- & -- & T & -- & -- & -- & - \\ -- & -- & T & -- & -- & -- & - \\ -- & -- & T & -- & -- & -- & -\end{array}$

$\begin{array}{lcllllll}3.2 & 01-12-78 & 0 & -- & -- & -- & -- & \\ 2 & 01-27-78 & 0 & -- & -- & -- & -- & \\ 5.3 & 02-09-78 & 0 & -- & -- & -- & -- & \text { NEARBY WELL WAS BEING PUMPED } \\ -- & -- & \text { IN } & 30 & -- & -- & -- & \text { BEDROCK } \\ 7.5 & 03-00-78 & \text { H } & -- & -- & -- & -- & \text { RECENTLY PUMPED WELL } \\ 9.2 & 04-00-78 & \text { H } & -- & -- & -- & -- & \text { RECENTLY PUMPED WELL } \\ 1 & 05-00-78 & \text { H } & -- & -- & -- & -- & \text { RECENTLY PUMPED WELL }\end{array}$




\begin{tabular}{|c|c|c|c|c|c|c|c|c|c|c|c|}
\hline & & & & & Altitude & Diam- & Depth to & & & $\begin{array}{l}\text { Depth to } \\
\text { bottom }\end{array}$ & \\
\hline $\begin{array}{l}\text { Local } \\
\text { site } \\
\text { number }\end{array}$ & $\begin{array}{l}\text { Lati- } \\
\text { tude }\end{array}$ & $\begin{array}{l}\text { Long- } \\
\text { tude }\end{array}$ & Owner or user & $\begin{array}{l}\text { Year } \\
\text { comp- } \\
\text { leted }\end{array}$ & $\begin{array}{c}\text { above } \\
\text { sea } \\
\text { level } \\
\text { (ft) }\end{array}$ & $\begin{array}{l}\text { eter } \\
\text { of } \\
\text { well } \\
\text { (in.) }\end{array}$ & $\begin{array}{c}\text { bottom } \\
\text { of } \\
\text { casing } \\
\text { (ft) }\end{array}$ & $\begin{array}{l}\text { Casing } \\
\text { mate- } \\
\text { rial }\end{array}$ & $\begin{array}{l}\text { Type } \\
\text { of } \\
\text { fin- } \\
\text { ish }\end{array}$ & $\begin{array}{l}\text { of } \\
\text { open } \\
\text { section } \\
\text { (ft) }\end{array}$ & $\begin{array}{l}\text { Type } \\
\text { of } \\
\text { site }\end{array}$ \\
\hline
\end{tabular}

Candia--Continued

$\begin{array}{rrrr}\text { CDW } & 8 & 430458 & 711840 \\ \text { CDW } & 9 & 430447 & 711732 \\ & & & \\ \text { CDW } & 10 & 430439 & 711647 \\ \text { CDW } & 11 & 430430 & 711549 \\ \text { CDW } & 12 & 430437 & 711838 \\ \text { CDW } & 13 & 430400 & 711703 \\ \text { CDW } & 14 & 430337 & 711641 \\ \text { CDW } & 15 & 430159 & 711527\end{array}$

CLA $\quad 1 \quad 425830 \quad 711151$

$\begin{array}{llll}\text { CLA } & 3 & 425921 & 711225\end{array}$

CLB $1425630 \quad 711429$

CLW $\quad 1 \quad 425732 \quad 711525$

CLW $2425919 \quad 711222$

$\begin{array}{llll}\text { CLW } & 3 & 425859 & 711639\end{array}$

CLW $\quad 4 \quad 425919 \quad 711215$

$\begin{array}{llll}\text { CLW } & 8 & 425842 & 711150\end{array}$

CLW $\quad 9 \quad 425820 \quad 711152$

$\begin{array}{llll}C L W & 10 & 425922 & 711149\end{array}$

CLW $11 \quad 425919 \quad 711154$

$\begin{array}{llll}\text { CLW } & 13 & 425907 & 711217\end{array}$

CLW $\quad 14 \quad 425903 \quad 711219$

CLW $15 \quad 425900 \quad 711212$

$\begin{array}{llll}\text { CLW } & 17 & 425842 & 711303\end{array}$

CLW $\quad 18 \quad 425829 \quad 711304$

$\begin{array}{llll}\text { CLW } & 19 & 425834 & 711253\end{array}$

CLW $\quad 20 \quad 425848 \quad 711241$

$\begin{array}{llll}\text { CLW } & 21 & 425849 & 711248\end{array}$

$\begin{array}{llll}\text { CLW } & 22 & 425850 & 711244\end{array}$

$\begin{array}{llll}\text { CLW } & 23 & 425826 & 711300\end{array}$

CLW $\quad 24 \quad 425825 \quad 711250$

CLW $\quad 25 \quad 425805 \quad 711252$

$\begin{array}{llll}\text { CLW } & 26 & 425811 & 711250\end{array}$

$\begin{array}{llll}C L W & 28 & 425755 & 711246\end{array}$

CLW $29 \quad 425802 \quad 711201$

CLW $30 \quad 425804 \quad 711159$

$\begin{array}{llll}\text { CLW } & 31 & 425808 & 711151\end{array}$

$\begin{array}{llll}\text { CLW } & 32 & 425820 & 711115\end{array}$

$\begin{array}{llll}C L W & 33 & 425815 & 711111\end{array}$

CLW $34 \quad 425819 \quad 711105$

CLW $\quad 35 \quad 425820 \quad 711056$

$\begin{array}{llll}\text { CLW } & 37 & 425918 & 711140\end{array}$

$\begin{array}{lllll}\text { CLW } & 38 & 425916 & 711137\end{array}$

CLW $39 \quad 425914 \quad 711114$

CLW $40 \quad 425914 \quad 711138$

$\begin{array}{llll}\text { CLW } & 41 & 425917 & 711217\end{array}$

WATSON
WALEGA
MANN JESSE
SCARLETT HOMES
STACY
DESJARDINS
HILLSIDE INC

$\begin{array}{lrrrrrrr}1985 & 530 & -- & -- & -- & X & -- & \text { BrW } \\ 1985 & 340 & -- & -- & -- & X & -- & \text { BrW } \\ & & & & & & & \\ 1986 & 320 & 6 & 35.0 & \text { S } & -- & -- & \text { BrW } \\ 1985 & 410 & 7 & 20.0 & S & -- & -- & \text { BrW } \\ 1985 & 420 & 7 & 36.0 & S & -- & -- & \text { BrW } \\ 1984 & 430 & -- & -- & -- & X & -- & \text { BrW } \\ 1985 & 450 & -- & -- & -- & X & -- & \text { BrW } \\ 1985 & 400 & -- & -- & -- & X & -- & \text { BrW }\end{array}$

\section{Chester}

\begin{tabular}{|c|c|c|c|c|c|c|c|c|}
\hline-- & 1984 & 200 & -- & -- & -- & - & -- & Bor \\
\hline CHESTER, TOWN OF & 1985 & 180 & -- & -- & -- & -- & - & Bor \\
\hline NHDOT & 1974 & 307 & -- & -- & -- & -- & -- & Wsh \\
\hline WARREN ALBERT & 1966 & 450 & 48 & 10.0 & T & -- & -- & Dug \\
\hline CHESTER, TOWN OF & 1985 & 185 & -- & -- & -- & -- & -- & Bor \\
\hline GOTT CHESTER & 1965 & 480 & 6 & 3.0 & $\mathrm{~s}$ & $\mathrm{x}$ & 250.0 & BrW \\
\hline CHESTER, TOWN OF & 1985 & 181.1 & -- & -- & -- & o & -- & Bor \\
\hline -- & 1984 & 176 & 2 & 11.3 & $\mathrm{P}$ & P & 13.3 & Bor \\
\hline -- & 1984 & 200 & 2 & 26.5 & $\mathrm{P}$ & $\mathrm{P}$ & 28.5 & Bor \\
\hline DUNN A & 1971 & 170 & 8 & 28.0 & $\mathrm{~s}$ & $\mathrm{x}$ & -- & $\mathrm{BrW}$ \\
\hline SIMANO & 1972 & 180 & 54 & 24.0 & $T$ & 0 & -- & Dug \\
\hline DROWNE RALPH & 1980 & 190 & 36 & 12.0 & $\mathrm{~T}$ & 0 & -- & Dug \\
\hline GRAHAM & 1976 & 190 & -- & -- & -- & $\mathrm{x}$ & -- & $\mathrm{BrW}$ \\
\hline DROWNE $R$ JR & 1977 & 180 & 36 & 9.0 & $T$ & 0 & -- & Dug \\
\hline CASH & 1956 & 200 & 48 & 20.0 & T & 0 & -- & Dug \\
\hline CHURCH M & 1978 & 230 & 48 & 12.0 & T & 0 & -- & Dug \\
\hline BAT JER & 1958 & 200 & -- & -- & -- & 0 & -- & Dug \\
\hline BETHVEN & 1964 & 210 & 42 & 12.0 & $\mathrm{R}$ & 0 & -- & Dug \\
\hline DENNIS & 1974 & 195 & -- & -- & -- & 0 & -- & Dug \\
\hline DENNIS & 1961 & 200 & 48 & 16.0 & -- & -- & -- & Dug \\
\hline LANGILLE L G & 1954 & 230 & 108 & 5.0 & $\mathrm{R}$ & 0 & -- & Dug \\
\hline LANGILLE L G & 1933 & 210 & 48 & 18.0 & $\mathrm{R}$ & 0 & -- & Dug \\
\hline TOWLE J JR & 1952 & 205 & 18 & 6.7 & $\mathrm{~T}$ & 0 & -- & Dug \\
\hline PANDOLPHIN & 1960 & 215 & -- & -- & -- & 0 & -- & Dug \\
\hline COOPER & 1971 & 220 & 48 & 10.9 & C & 0 & -- & Dug \\
\hline THOMP SON & 1978 & 200 & -- & -- & -- & $\mathrm{x}$ & -- & $\mathrm{BrW}$ \\
\hline COMEAU JOHN & 1959 & 200 & -- & -- & -- & $\mathrm{x}$ & -- & $\mathrm{BrW}$ \\
\hline BRITON T & 1952 & 175 & 24 & 12.0 & $\mathrm{~T}$ & 0 & -- & Dug \\
\hline SCHMIDT D & 1930 & 190 & 48 & 15.0 & $\mathrm{R}$ & 0 & -- & Dug \\
\hline CHASE & $19 \pi 4$ & 215 & -- & -- & -- & $\mathrm{x}$ & -- & $\mathrm{BrW}$ \\
\hline DANIEL $T$ & 1982 & 200 & -- & -- & -- & -- & -- & Dug \\
\hline SIMP SON & 1973 & 190 & -- & -- & -- & $x$ & -- & $\mathrm{BrW}$ \\
\hline DUPRIS T & 1979 & 165 & 48 & 25.0 & $\mathrm{R}$ & 0 & -- & \\
\hline-- & 1976 & 165 & -- & -- & -- & -- & -- & Dvn \\
\hline HIDEAWAY FARM & 1976 & 185 & 6 & 30.0 & $s$ & $\mathrm{x}$ & -- & $\mathrm{Brw}$ \\
\hline MCCORMICK & 1979 & 165 & -- & -- & -- & $\mathrm{x}$ & -- & $\mathrm{BrW}$ \\
\hline CHESTER, TOWN OF & 1985 & 190.4 & -- & -- & -- & 0 & -- & \\
\hline
\end{tabular}




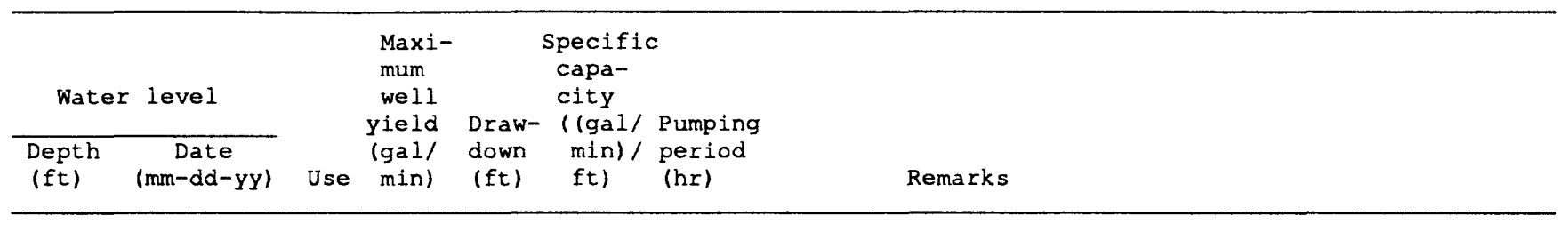

Candia--Continued

$\begin{array}{ccccccc}30 & 10-19-85 & \mathrm{H} & 25 & -- & -- & 2 \\ 8 & 09-14-85 & \mathrm{H} & 5 & -- & -- & .2 \\ -- & -- & \mathrm{H} & 6 & -- & -- & -- \\ -- & -- & \mathrm{H} & 3 & -- & -- & .5 \\ -- & -- & \mathrm{H} & 3.5 & -- & -- & .5 \\ 5 & 04-21-84 & \mathrm{H} & 2 & -- & -- & .5 \\ 6 & 04-25-85 & \mathrm{H} & 2 & -- & -- & .5 \\ -- & -- & \mathrm{H} & 8 & -- & -- & 1\end{array}$

Chester

$\begin{array}{lllllll}-- & -- & \text { T } & -- & -- & -- & -- \\ -- & -- & \text { T } & -- & -- & -- & --\end{array}$

USGS

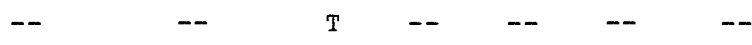

$2.208-14-68 \quad \mathrm{H} \quad--\quad--\quad--$

$\begin{array}{ccccccc}-- & -- & 0 & -- & -- & -- & -- \\ 1 & 00-00-65 & \text { H } & 3 & -- & -- & --\end{array}$

$\begin{array}{ccccccc}11 & 11-09-84 & 0 & -- & -- & -- & - \\ 20.4 & 11-09-84 & 0 & -- & -- & -- & - \\ -- & -- & \text { H } & 30 & -- & -- & - \\ -- & -- & \text { H } & -- & -- & -- & -- \\ -- & -- & \text { H } & -- & -- & -- & --\end{array}$

USGS

USGS CA

$\begin{array}{cllllll}15 & 01-01-76 & \text { H } & 1.5 & -- & -- & -- \\ 5.5 & 01-01-77 & \text { H } & 4 & -- & -- & - \\ 15 & 01-01-56 & \text { H } & -- & -- & -- & - \\ -- & 01-01-78 & \text { H, I } & -- & -- & -- & -\end{array}$

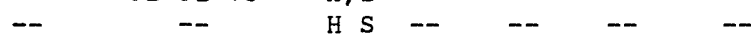

WATER TESTED BY WSPCC

7 01-01-64 H $\quad$-- $\quad--\quad--$

$\begin{array}{ccccccc}7 & 01-01-64 & \text { H } & -- & -- & -- & - \\ -- & -- & \text { H, T } & -- & -- & -- & - \\ -- & - & \text { U } & -- & -- & -- & -\end{array}$

$\begin{array}{lclllll}4 & 01-01-54 & \mathrm{H}, \mathrm{S} 17 & -- & -- & - \\ -- & -- & \mathrm{U} & -- & -- & -- & -\end{array}$

WATER TESTED BY WSPCC

$\begin{array}{rllllll}1.5 & 01-01-52 & \mathrm{H} & 10 & -- & -- & --\end{array}$

WATER TESTED BY WSPCC

OWNER REPORTS IRON STAIN

$\begin{array}{ccccccc}3.8 & 07-17-84 & \mathrm{H} & -- & -- & -- & -- \\ -- & -- & \mathrm{H} & -- & -- & -- & --\end{array}$

WATER TESTED BY WSPCC, SLIGHT S ODOR, GOOD

WATER TESTED WSPCC, TRACE IRON

50 01-01-59 $\mathrm{H}$-- $\quad--$

$\begin{array}{cclllll}2 & 01-01-52 & \text { H,S } & -- & -- & -- & -- \\ 6 & 01-01-30 & \text { H } & -- & -- & -- & -- \\ -- & -- & \text { H } & -- & -- & -- & -- \\ 12 & 01-01-82 & \text { H } & -- & -- & -- & -- \\ -- & -- & \text { H } & -- & -- & -- & --\end{array}$

WATER TESTED BY WSPCC, HIGH IRON

WATER TESTED BY WSPCC

OWNER REPORTS HEAVY IRON STAIN

$\begin{array}{ccccccc}3.5 & 01-01-79 & \text { H } & -- & -- & -- & -- \\ -- & -- & \text { H } & -- & -- & -- & -- \\ -- & -- & \text { H, I } & -- & -- & -- & -- \\ -- & -- & \text { H } & -- & -- & -- & -- \\ 12.7 & 09-26-85 & 0 & -- & -- & -- & --\end{array}$

DOES NOT DRINK WATER, POOR TASTE

OWNER REPORTS NEVER DRY 
Table 2.--Description of wells, borings,

\begin{tabular}{|c|c|c|c|c|c|c|c|c|c|c|c|}
\hline $\begin{array}{l}\text { Local } \\
\text { site } \\
\text { number }\end{array}$ & $\begin{array}{l}\text { Lati- } \\
\text { tude }\end{array}$ & $\begin{array}{l}\text { Long- } \\
\text { tude }\end{array}$ & Owner or user & $\begin{array}{l}\text { Year } \\
\text { comp- } \\
\text { leted }\end{array}$ & $\begin{array}{l}\text { Altitude } \\
\text { above } \\
\text { sea } \\
\text { level } \\
\text { (ft) }\end{array}$ & $\begin{array}{l}\text { Diam- } \\
\text { eter } \\
\text { of } \\
\text { well } \\
\text { (in.) }\end{array}$ & $\begin{array}{c}\text { Depth to } \\
\text { bottom } \\
\text { of } \\
\text { casing } \\
\text { (ft) }\end{array}$ & $\begin{array}{l}\text { Casing } \\
\text { mate- } \\
\text { rial }\end{array}$ & $\begin{array}{l}\text { D } \\
\text { Type } \\
\text { of } \\
\text { fin- } \\
\text { ish }\end{array}$ & $\begin{array}{l}\text { Depth to } \\
\text { bottom } \\
\text { of } \\
\text { open } \\
\text { section } \\
\text { (ft) }\end{array}$ & $\begin{array}{l}\text { Type } \\
\text { of } \\
\text { site }\end{array}$ \\
\hline
\end{tabular}

Chester--Continued

$\begin{array}{llll}\text { CLW } & 42 & 425917 & 711215 \\ \text { CLW } & 43 & 425921 & 711221 \\ \text { CLW } & 44 & 425919 & 711215 \\ \text { CLW } & 45 & 425923 & 711223 \\ \text { CLW } & 46 & 425918 & 711213 \\ & & & \\ \text { CLW } & 47 & 425919 & 711220 \\ \text { CLW } & 48 & 425922 & 711215 \\ \text { CLW } & 49 & 425922 & 711215 \\ \text { CLW } & 50 & 425917 & 711224\end{array}$

CHESTER, TOWN OF CHESTER, TOWN OF CHESTER, TOWN OF CHESTER, TOWN OF CHESTER， TOWN OF

CHESTER, TOWN OF CHESTER, TOWN OF CHESTER, TOWN OF CHESTER, TOWN OF

$\begin{array}{llllllll}1985 & 186.2 & -- & -- & -- & 0 & -- & \text { Bor } \\ 1985 & 183.0 & -- & -- & -- & 0 & -- & \text { Bor } \\ 1985 & 181.2 & -- & -- & -- & 0 & -- & \text { Bor } \\ 1985 & 170.3 & -- & -- & -- & 0 & -- & \text { Bor } \\ 1985 & 176.7 & -- & -- & -- & 0 & -- & \text { Bor } \\ & & & & & & & \\ 1985 & 173.8 & -- & -- & -- & 0 & -- & \text { Bor } \\ 1985 & 173.9 & -- & -- & -- & 0 & -- & \text { Bor } \\ 1985 & 170.4 & -- & -- & -- & 0 & -- & \text { Bor } \\ 1985 & 190.4 & -- & -- & -- & 0 & -- & \text { Bor }\end{array}$

Deerfield

$\begin{array}{ll}1984 & 260 \\ 1973 & 418 . \\ -- & 275 \\ -- & 280 \\ 1971 & 275 \\ 1977 & 595 \\ 1972 & 550 \\ 1977 & 325 \\ 1978 & 228 \\ 1978 & 825 \\ 1955 & 460 \\ 1964 & 460 \\ 1978 & 470 \\ 1978 & 430 \\ 1980 & 430 \\ 1980 & 430 \\ 1979 & 430 \\ 1977 & 435 \\ & \\ 1981 & 440 \\ 1955 & 430 \\ 1981 & 430 \\ 1975 & 440 \\ 1979 & 375 \\ 1950 & 310 \\ 1976 & 310 \\ 1977 & 315 \\ 1934 & 320 \\ 1966 & 325 \\ 1964 & 320 \\ 1973 & 265 \\ 1974 & 265 \\ 1937 & 235 \\ 1970 & 265\end{array}$

\begin{tabular}{|c|c|c|c|c|}
\hline-- & -- & -- & -- & -- \\
\hline-- & - & -- & -- & -- \\
\hline-- & - & -- & -- & -- \\
\hline-- & -- & -- & -- & - \\
\hline 6 & 55.0 & $s$ & G & 59.0 \\
\hline 36 & 11.3 & $\mathrm{R}$ & W & 11.3 \\
\hline 36 & 31.2 & $\mathrm{R}$ & W & 31.2 \\
\hline 36 & 21.7 & $R$ & W & 21.7 \\
\hline 24 & 12.0 & C & 0 & 12.0 \\
\hline 36 & 14.5 & $\mathrm{R}$ & W & 14.5 \\
\hline-- & 25.0 & -- & $\mathrm{x}$ & 208.0 \\
\hline 36 & 6.0 & $\mathrm{~T}$ & 0 & -- \\
\hline-- & - & -- & 0 & - \\
\hline-- & -- & -- & 0 & -- \\
\hline 36 & 90.0 & C & 0 & -- \\
\hline-- & - & -- & 0 & -- \\
\hline-- & -- & -- & 0 & - \\
\hline- & -- & -- & 0 & -- \\
\hline
\end{tabular}

$\begin{array}{cc}36 & 10.0 \\ 24 & 8.7 \\ 36 & 14.0 \\ 36 & 16.0 \\ -- & -- \\ & \\ 8 & 12.0 \\ 8 & 12.0 \\ -- & -- \\ -- & -- \\ 36 & 12.0 \\ 8 & -- \\ 8 & 80.0 \\ -- & -- \\ -- & -- \\ 48 & 15.0\end{array}$

$\begin{array}{rrrr}\text { B } & -- & -- & \text { Dug } \\ T & 0 & -- & \text { Dug } \\ \text { C } & 0 & -- & \text { Dug } \\ \text { C } & 0 & -- & \text { Dug } \\ -- & 0 & -- & \text { Dug } \\ & & & \\ \text { S } & 0 & -- & \text { Cbl } \\ \text { S } & 0 & -- & \text { BrW } \\ -- & 0 & -- & \text { BrW } \\ -- & 0 & -- & \text { CbI } \\ \text { C } & 0 & -- & \text { Dug } \\ & & & \\ \text { S } & -- & -- & \text { BrW } \\ \text { S } & -- & -- & \text { BrW } \\ -- & 0 & -- & \text { BrW } \\ -- & 0 & -- & \text { Dug } \\ \text { C } & 0 & -- & \text { Dug }\end{array}$ 


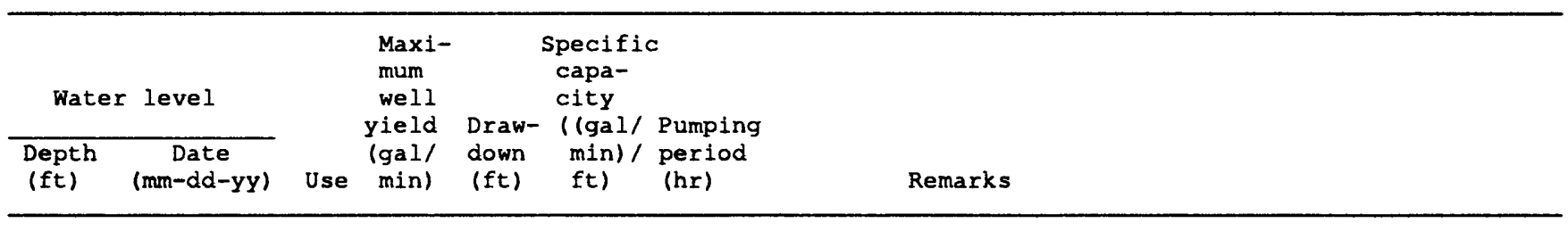

\begin{tabular}{|c|c|c|c|c|c|c|c|}
\hline \multicolumn{8}{|c|}{ Chester--Continued } \\
\hline 10.5 & $09-26-85$ & 0 & -- & -- & -- & -- & \\
\hline- & -- & 0 & -- & -- & -- & -- & DRY WELL \\
\hline 7.2 & $09-26-85$ & 0 & -- & -- & -- & -- & \\
\hline 2.2 & $09-26-85$ & 0 & -- & -- & -- & -- & \\
\hline 9 & $09-26-85$ & 0 & -- & -- & -- & -- & \\
\hline 3.1 & $09-26-85$ & 0 & -- & -- & -- & -- & \\
\hline 6.4 & $09-26-85$ & 0 & -- & -- & -- & -- & \\
\hline 3.5 & $09-26-85$ & 0 & -- & -- & -- & -- & \\
\hline 8.2 & $09-26-85$ & 0 & -- & -- & -- & -- & \\
\hline \multicolumn{8}{|c|}{ Deerfield } \\
\hline-- & -- & $\mathbf{T}$ & -- & -- & -- & -- & USGS \\
\hline-- & - & $\mathbf{T}$ & -- & -- & -- & -- & \\
\hline-- & - & $\mathrm{H}$ & -- & -- & -- & -- & \\
\hline-- & - & H & -- & -- & -- & -- & \\
\hline 20.6 & $00-00-71$ & $\mathrm{P}$ & 50 & -- & 7.1 & -- & RECENTLY PUMPED WELL \\
\hline 3.6 & $01-12-78$ & 0 & -- & -- & -- & -- & \\
\hline 10.3 & $01-01-78$ & 0 & -- & -- & -- & -- & \\
\hline 14.5 & $08-00-77$ & $\mathrm{U}$ & -- & -- & -- & -- & \\
\hline 7 & $02-00-78$ & $\mathrm{p}$ & -- & -- & -- & -- & \\
\hline 8.3 & $04-00-78$ & $\mathbf{H}$ & -- & -- & -- & -- & \\
\hline-- & -- & IN & 50 & -- & -- & -- & \\
\hline 2 & $00-00-64$ & $\mathrm{H}, \mathrm{I}$ & -- & -- & -- & -- & $\begin{array}{l}\text { WATER SERVES } 3 \text { HOMES AND IS ALSO USED FOR BUSINESS } \\
\text { WELL IS FLOWING AT TIMES }\end{array}$ \\
\hline 30 & $00-00-78$ & $\mathrm{H}$ & 7 & -- & -- & -- & WATER TESTED BY WSPCC \\
\hline-- & -- & H & 50 & -- & -- & -- & OWNER REPORTS BEDROCK AT 60 FEET \\
\hline-- & -- & $\mathrm{H}$ & -- & -- & -- & -- & \\
\hline-- & -- & $\mathrm{H}$ & -- & -- & -- & -- & \\
\hline-- & -- & $\mathrm{H}$ & -- & -- & -- & -- & REPORTED BY RENTER \\
\hline 4 & $05-03-84$ & $\mathrm{H}$ & -- & -- & -- & -- & $\begin{array}{l}\text { WATER TESTED BY WSPCC, IRON STAIN, WENT DRY IN THE } \\
\text { SUMMER OF } 1983\end{array}$ \\
\hline-- & -- & $\mathrm{H}$ & -- & -- & -- & -- & WHEN RAINS WATER SMELLS AND TASTES BAD, IRON STAIN \\
\hline 3.9 & $05-31-84$ & $\mathrm{H}$ & 25 & -- & -- & -- & WATER TESTED BY WSPCC \\
\hline-- & -- & $\mathrm{H}$ & -- & -- & -- & -- & \\
\hline-- & -- & $\mathrm{H}$ & -- & -- & -- & -- & FLOWING WELL, WATER TESTED BY WATER TEST INC \\
\hline 6.6 & $06-04-84$ & $\mathrm{H}$ & -- & -- & -- & -- & \\
\hline-- & -- & $\mathrm{U}$ & -- & -- & -- & -- & OWNER REPORTS 10-12 FEET TO BEDROCK \\
\hline-- & -- & $\mathrm{H}$ & -- & -- & -- & -- & WATER TESTED BY WSPCC, TRACE IRON, SOFTENER USED \\
\hline-- & -- & $\mathrm{H}$ & -- & -- & -- & -- & \\
\hline-- & -- & H & -- & -- & -- & -- & WATER TESTED WSPCC \\
\hline-- & -- & $\mathrm{H}, \mathrm{S}$ & -- & -- & -- & -- & WATER TESTED BY WSPCC \\
\hline-- & -- & $\mathrm{H}$ & -- & -- & -- & -- & \\
\hline 6 & $00-00-73$ & $\mathrm{H}, \mathrm{I}$ & -- & -- & -- & -- & \\
\hline-- & -- & $\mathrm{H}$ & -- & -- & -- & -- & \\
\hline $\begin{array}{l}3 \\
5\end{array}$ & $\begin{array}{l}00-00-37 \\
06-05-84\end{array}$ & $\begin{array}{l}\mathrm{H} \\
\mathrm{H}\end{array}$ & -- & -- & -- & -- & \\
\hline
\end{tabular}


Table 2.--Description of wells, borings,

\begin{tabular}{llllll}
\hline & & & & Depth to \\
bottom \\
Local
\end{tabular}

Deerfield--Continued

\begin{tabular}{|c|c|c|c|c|c|c|c|c|c|c|c|c|}
\hline DDW & 37 & 430525 & 711439 & HIGGINS & 1981 & 280 & 36 & 12.0 & C & 0 & -- & Dug \\
\hline DDW & 41 & 430503 & 711357 & AMOR E & 1977 & 225 & -- & -- & -- & - & -- & Dug \\
\hline DDW & 42 & 430505 & 711352 & RIEL G & 1967 & 230 & 48 & 20.0 & $\mathrm{~T}$ & 0 & -- & Dug \\
\hline DDW & 43 & 430929 & 711411 & STEVENS HAL & 1981 & 430 & 6 & 22.0 & s & 0 & -- & $\mathrm{BrW}$ \\
\hline DDW & 44 & 430558 & 711452 & -- & 1984 & 276 & 2 & 65.6 & $\mathrm{P}$ & $\mathrm{P}$ & 67.6 & Bor \\
\hline DDW & 45 & 430632 & 711617 & -- & 1984 & 268 & 2 & 12.0 & $\mathrm{P}$ & $\mathrm{P}$ & 14.0 & Bor \\
\hline DDW & 48 & 430500 & 711335 & YOUNG & 1984 & 280 & -- & -- & -- & $\mathrm{x}$ & -- & $\mathrm{BrW}$ \\
\hline DDW & 49 & 430506 & 711338 & BROWN & 1984 & 300 & -- & -- & - & $x$ & -- & $\mathrm{BrW}$ \\
\hline DDW & 50 & 430524 & 711439 & SOLAK & 1985 & 280 & -- & -- & -- & $\mathrm{x}$ & - & $\mathrm{BrW}$ \\
\hline DDW & 51 & 430518 & 711454 & CLARK & 1985 & 390 & -- & - & -- & $\mathrm{x}$ & - & $\mathrm{BrW}$ \\
\hline DDW & 52 & 430604 & 711353 & CHALBECK & 1985 & 350 & -- & -- & -- & $x$ & -- & $\mathrm{BrW}$ \\
\hline DDW & 58 & 430831 & 711354 & DEVLIN & 1985 & 410 & - & -- & - & $\mathrm{x}$ & -- & $\mathrm{Brw}$ \\
\hline DDW & 59 & 430747 & 711430 & DUHAIME & 1985 & 380 & - & -- & -- & $\mathrm{x}$ & - & $\mathrm{BrW}$ \\
\hline DDW & 60 & 430745 & 711439 & FRAZIER & 1985 & 360 & -- & -- & -- & $\mathrm{x}$ & -- & $\mathrm{BrW}$ \\
\hline DDW & 61 & 430733 & 711455 & LINDEN & 1984 & 360 & -- & -- & -- & $\mathrm{x}$ & -- & Brw \\
\hline DDW & 62 & 430859 & 711347 & ALIE & 1984 & 460 & -- & -- & - & $\mathrm{x}$ & -- & Brw \\
\hline DDW & 63 & 430849 & 711329 & BURKLUND & 1986 & 470 & -- & 51.0 & -- & $\mathrm{x}$ & - & $\mathrm{BrW}$ \\
\hline DDW & 64 & 430941 & 711441 & GRANT & 1985 & 530 & -- & 19.0 & -- & $\mathrm{x}$ & -- & $\mathrm{BrW}$ \\
\hline DDW & 65 & 430950 & 711440 & SAWYER & 1984 & 560 & -- & -- & -- & $\mathrm{x}$ & -- & $\mathrm{BrW}$ \\
\hline DDW & 66 & 430529 & 711531 & DODGE & 1985 & 480 & -- & -- & - & $x$ & - & $\mathrm{BrW}$ \\
\hline
\end{tabular}

East Kingston

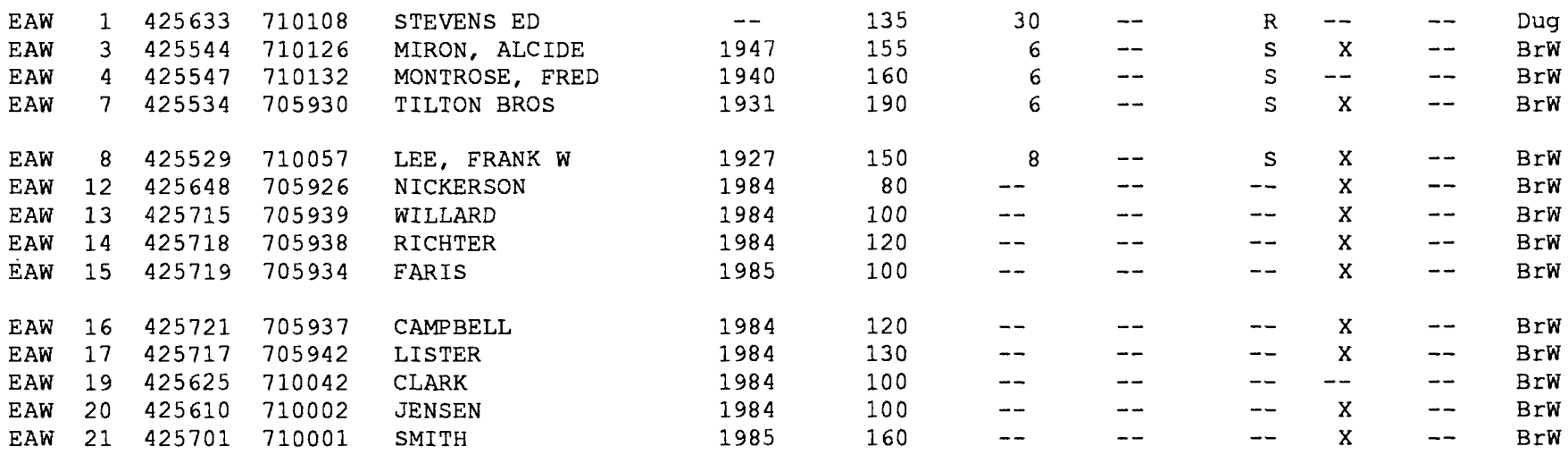




\section{and springs--Continued}

\begin{tabular}{|c|c|c|c|c|c|c|c|}
\hline Wate & I level & & $\begin{array}{l}\text { Maxi- } \\
\text { mum } \\
\text { well } \\
\text { yield }\end{array}$ & $\begin{array}{r}5 \\
\text { Draw- }\end{array}$ & $\begin{array}{l}\text { Specific } \\
\text { capa- } \\
\text { city } \\
\text { (lgal/ }\end{array}$ & $\begin{array}{l}\text { c } \\
\text { Pumping }\end{array}$ & \\
\hline
\end{tabular}

\section{Deerfield--Continued}

$\begin{array}{llllllll}-- & -- & H & -- & -- & -- & -- & \text { REPORTED BY RENTER } \\ -- & -- & H & -- & -- & -- & -- & \\ -- & -- & \text { H } & -- & -- & -- & -- & \\ -- & -- & \text { H } & -- & -- & -- & -- & \\ -- & -- & \text { H } & -- & -- & -- & -- & \text { WATER TESTED BY WSPCC, TRACE IRON }\end{array}$

$\begin{array}{rlllllll}220 & 00-00-81 & \text { H } & 1.5 & -- & -- & -- & \\ 23.5 & 11-06-84 & 0 & -- & -- & -- & -- & \text { USGS, CA } \\ 4.6 & 11-06-84 & 0 & -- & -- & -- & -- & \text { USGS, CA }\end{array}$

$\begin{array}{rrllllll}4.6 & 11-06-84 & 0 & -- & -- & -- & -- & \text { USGS, CA } \\ 38.9 & 11-06-84 & 0 & -- & -- & -- & -- & \text { USGS, CA }\end{array}$

29 11-06-84 0 -- $\quad--\quad--\quad--\quad$ USGS

$30 \quad 10-23-84$

$55 \quad 07-26-84$

$20 \quad 08-\overline{0-8}-8$

(1)

$7 \quad 02-27-8$

$-$

$--$

$--\quad--$

$7 \quad 10-18-85$

$10-18-85$
$07-25-85$

$\begin{array}{ll}12 & 07-29-85 \\ 30 & 05-17-84\end{array}$

$\begin{array}{rr}12 & 07-29-85 \\ 30 & 05-17-84 \\ 8 & 11-26-84\end{array}$

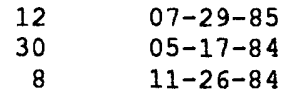

$\begin{array}{ll}-- & -- \\ -- & -- \\ -- & --\end{array}$

$\begin{array}{llll}1 & - & - & .2\end{array}$

$\begin{array}{llll}1.5 & - & - & 1.5 \\ 0.3 & -- & - & 1.5\end{array}$

$\begin{array}{lllll}\mathrm{H} & 0.3 \quad- & - & 1.5\end{array}$

$\begin{array}{lllll}\mathrm{H} & 2 & -- & -- & -- \\ \mathrm{H} & 1 & -- & -- & .\end{array}$

$\begin{array}{rrrrr}\mathrm{H} & 4 & -- & -- & -- \\ \mathrm{H} & 30 & -- & -- & \end{array}$

H 15 --

$\begin{array}{ccccc}\text { H } & 14 & -- & -- & 1 \\ \text { H } & 4 & -- & -- & .2 \\ \text { H } & 1.8 & -- & -- & .2 \\ \text { H } & 30 & -- & -- & -. \\ \text { H } & 4 & -- & -- & .2 \\ & & & & \\ \text { H } & 50 & -- & -- & .2 \\ \text { H } & 20 & -- & -- & .5 \\ \text { H } & 2.5 & -- & -- & .1 \\ \text { H } & 100 & -- & -- & 2\end{array}$

East Kingston

$\begin{array}{lclclll}11.9 & 10-25-55 & \text { U } & -- & -- & -- & -- \\ 20 & 01-01-47 & \text { H } & -- & -- & -- & -- \\ -- & -- & \text { H } & -- & -- & -- & -- \\ -- & -- & \text { H } & 15 & -- & -- & -- \\ -- & -- & \text { H } & 20 & -- & -- & -- \\ 15 & 07-23-84 & \text { H } & 25 & -- & -- & -- \\ -- & -- & \text { H } & 2 & -- & -- & -- \\ 7 & 09-01-84 & \text { H } & 5 & -- & -- & 1 \\ -- & -- & \text { H } & 20 & -- & -- & -- \\ -- & -- & \text { H } & 20 & -- & -- & -- \\ -- & -- & \text { H } & 0.8 & -- & -- & -- \\ 6 & 02-22-85 & \text { H } & -- & -- & -- & -- \\ 8 & 06-21-84 & \text { H } & 15 & -- & -- & -2 \\ -- & -- & \text { H } & 20 & -- & -- & --\end{array}$


Table 2.--Description of wells, borings,

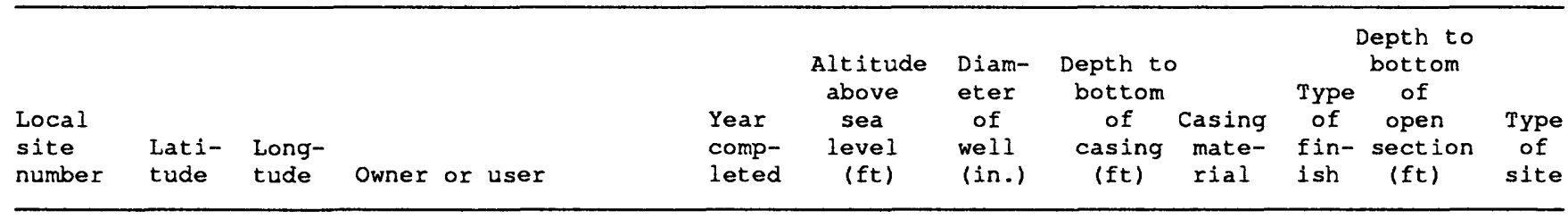

\section{Epping}

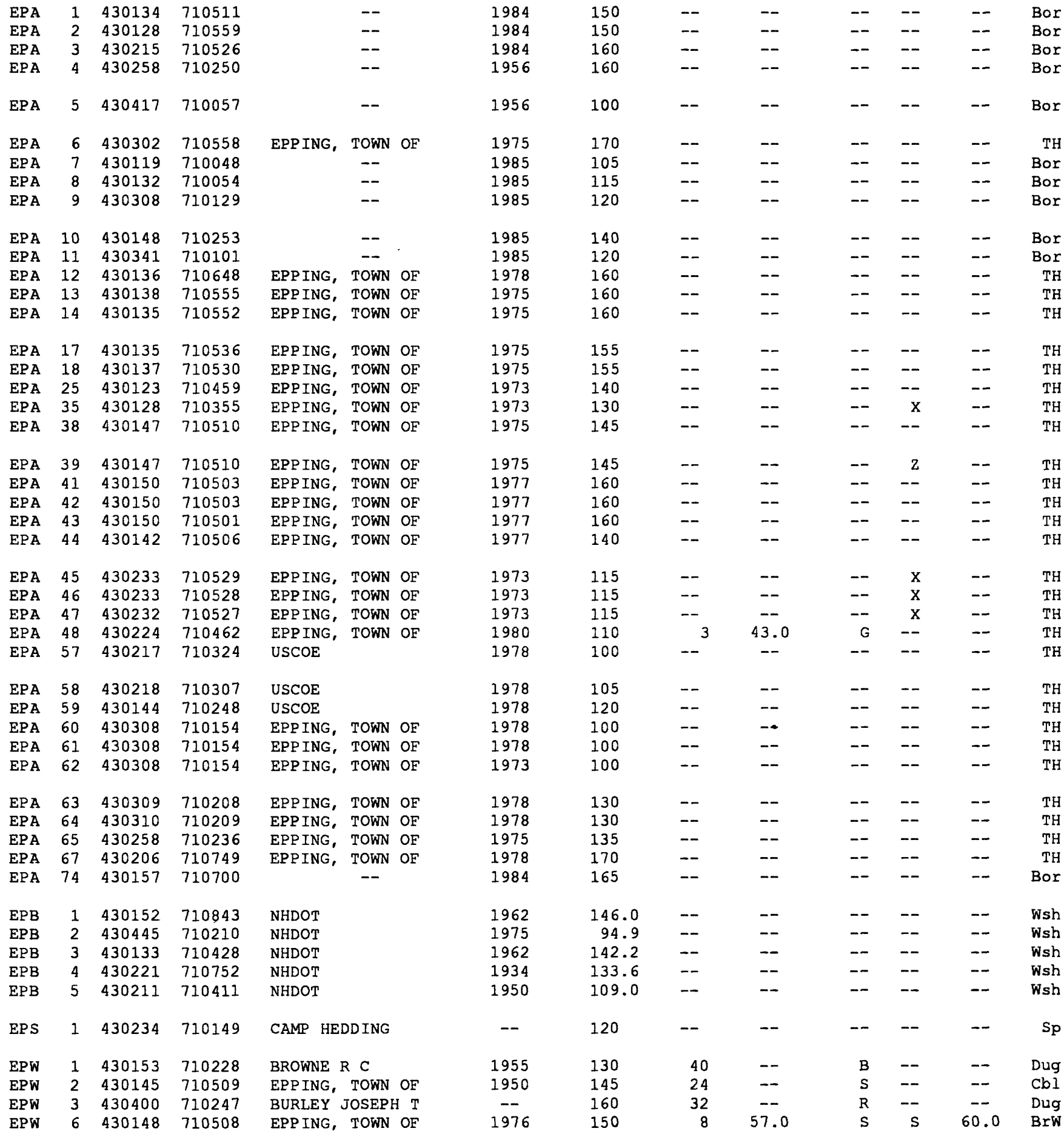




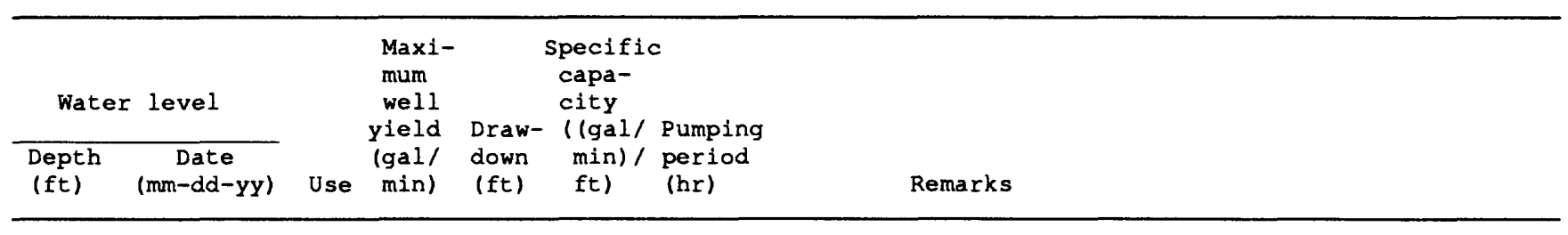

\section{Epping}

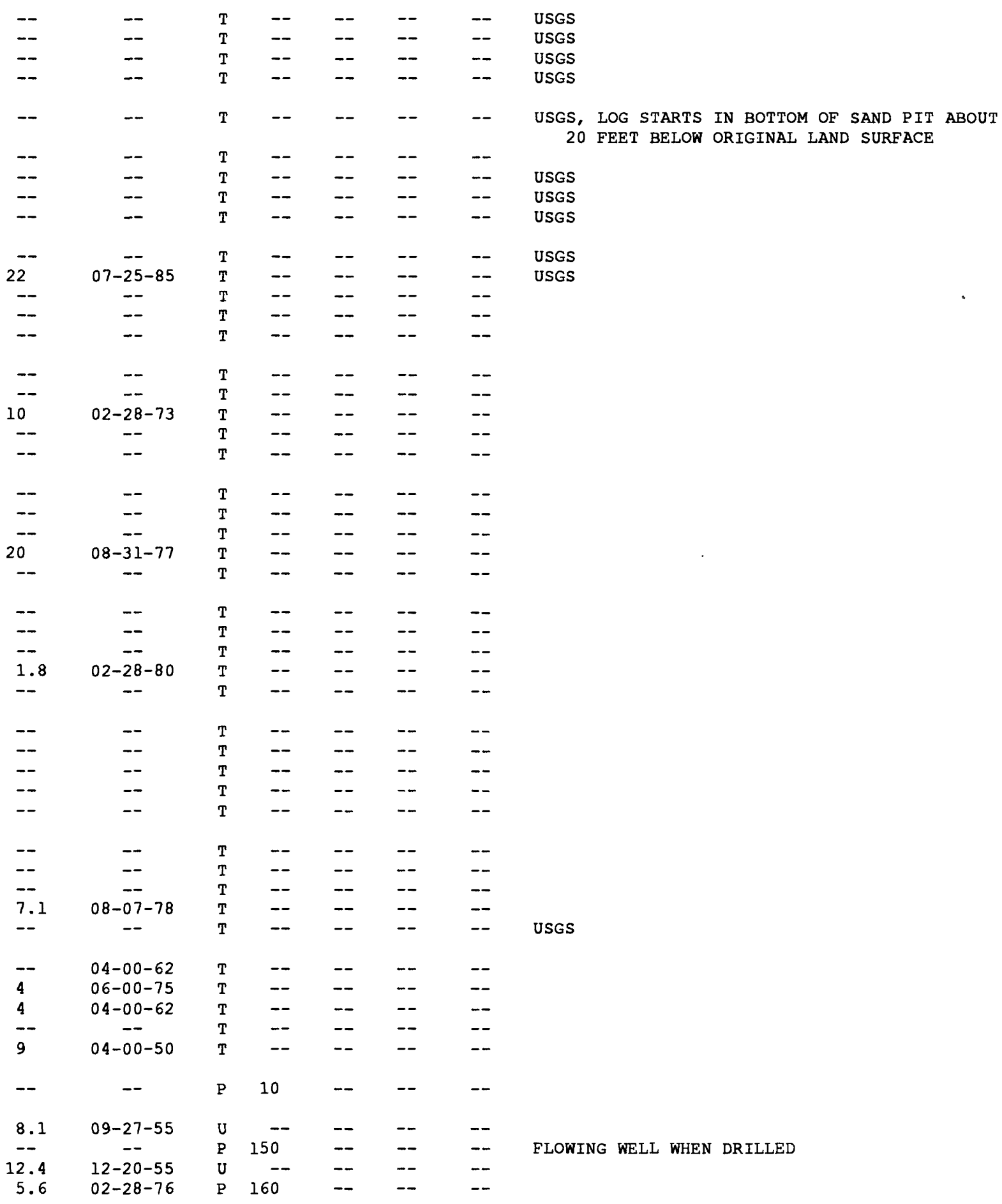




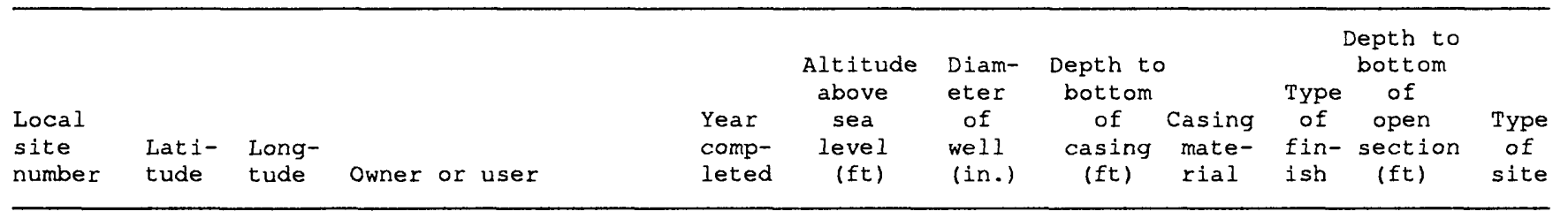

Epping--Cont inued

\begin{tabular}{|c|c|c|c|c|c|c|c|c|c|c|c|c|}
\hline EPW & 7 & 430126 & 710503 & EPPING, TOWN OF & 1973 & 145 & 3 & 73.0 & $\mathrm{~s}$ & $\mathrm{~s}$ & 78.0 & Bor \\
\hline EPW & 10 & 430231 & 710140 & CAMP HEDDING & 1977 & 120 & 36 & 15.0 & $\mathrm{C}$ & 0 & 15.0 & Dug \\
\hline EPW & 11 & 430235 & 710349 & PINE \& POND MOBILE HM & 1968 & 155 & 6 & 18.0 & $\mathrm{~s}$ & $\mathrm{x}$ & 250.0 & Brw \\
\hline EPW & 13 & 430407 & 710055 & KOLES & 1970 & 110 & 2 & 18.0 & $\mathrm{~s}$ & $\mathrm{~T}$ & 20.0 & Dvn \\
\hline EPW & 14 & 430133 & 710702 & -- & 1978 & 170 & -- & -- & -- & 0 & 12.0 & Dug \\
\hline EPW & 15 & 430341 & 710737 & RASP ELLANOR & 1974 & 245 & -- & 20.0 & -- & $\mathrm{x}$ & 190.0 & BrW \\
\hline EPW & 18 & 430301 & 710555 & EPPING, TOWN OF & 1975 & 185 & 3 & 21.0 & $\mathrm{~s}$ & -- & 26.0 & Wsh \\
\hline EPW & 19 & 430159 & 710748 & EPPING, TOWN OF & 1978 & 155 & 3 & 32.0 & $\mathbf{s}$ & $\mathbf{s}$ & 37.0 & Wsh \\
\hline EPW & 20 & 430159 & 710750 & EPPING, TOWN OF & 1978 & 155 & 3 & 31.0 & $s$ & $\mathrm{~s}$ & 36.0 & Wsh \\
\hline EPW & 21 & 430159 & 710745 & EPPING， TOWN OF & 1978 & 155 & 3 & 31.0 & $s$ & $s$ & 36.0 & Wsh \\
\hline EPW & 22 & 430210 & 710750 & EPPING, TOWN OF & 1978 & 160 & 3 & 36.0 & $s$ & $s$ & 41.0 & Wsh \\
\hline EPW & 28 & 430147 & 710514 & EPPING, TOWN OF & 1975 & 145 & 3 & 56.0 & -- & -- & 56.0 & Wsh \\
\hline EPW & 29 & 430222 & 710547 & BYRNES LESLEY & 1965 & 190 & -- & 107.0 & -- & $\mathrm{x}$ & 208.0 & Brw \\
\hline EPW & 30 & 430218 & 710541 & LAVOIE THEODORE & 1965 & 170 & -- & 21.0 & -- & $\mathrm{x}$ & 104.0 & BrW \\
\hline EPW & 31 & 430225 & 710456 & EPPING, TOWN OF & 1980 & 110 & 3 & 29.0 & $\mathbf{s}$ & s & 34.0 & Wsh \\
\hline EPW & 32 & 430319 & 710451 & ELLOIT & 1967 & 215 & -- & 16.0 & -- & $\mathrm{x}$ & 80.0 & $\mathrm{BrW}$ \\
\hline EPW & 33 & 430212 & 710437 & LAVOIE JACK & 1971 & 140 & -- & -- & -- & $\mathrm{x}$ & -- & Brw \\
\hline EPW & 34 & 430206 & 710337 & MORRISON & 1968 & 145 & -- & -- & -- & $\mathrm{x}$ & -- & $\mathrm{BrW}$ \\
\hline EPW & 35 & 430155 & 710304 & CROCTEAU CHESTETER & 1971 & 165 & -- & -- & -- & $\mathrm{x}$ & -- & $\mathrm{BrW}$ \\
\hline EPW & 36 & 430131 & 710229 & KEACK DON & 1968 & 165 & -- & -- & -- & $\mathrm{x}$ & -- & Brw \\
\hline EPW & 37 & 430234 & 710112 & PHILLIPS NATHAN & 1965 & 130 & -- & 150.0 & -- & $\mathrm{x}$ & 230.0 & $\mathrm{BrW}$ \\
\hline EPW & 47 & 430127 & 710352 & EPPING, TOWN OF & 1973 & 125 & -- & -- & -- & -- & -- & Wsh \\
\hline EPW & 48 & 430149 & 710508 & EPPING, TOWN OF & 1975 & 110 & 3 & 51.0 & $\mathrm{~S}$ & - & 56.0 & Wsh \\
\hline EPW & 49 & 430226 & 710461 & EPPING, TOWN OF & 1980 & 110 & 3 & 38.0 & $\mathrm{~s}$ & $\mathrm{~s}$ & 43.0 & \\
\hline EPW & 50 & 430223 & 710456 & EPPING, TOWN OF & 1980 & 110 & 3 & 26.0 & $\mathbf{s}$ & $\mathrm{s}$ & 31.0 & \\
\hline EPW & 51 & 430134 & 710510 & EPPING， TOWN OF & 1979 & 150 & -- & -- & -- & -- & -- & Wsh \\
\hline EPW & 52 & 430130 & 710509 & EPPING, TOWN OF & 1979 & 150 & -- & -- & -- & -- & -- & Wsh \\
\hline EPW & 53 & 430129 & 710509 & EPPING, TOWN OF & 1979 & 150 & -- & -- & -- & -- & -- & Wsh \\
\hline EPW & 54 & 430127 & 710508 & EPP ING, TOWN OF & 1980 & 150 & -- & -- & -- & -- & -- & \\
\hline EPW & 55 & 430234 & 710115 & -- & 1985 & 128 & 2 & 17.8 & $\mathrm{P}$ & $\mathrm{P}$ & 20.3 & $\mathrm{~B}$ \\
\hline EPW & 58 & 430241 & 710735 & BENNETT & 1974 & 160 & -- & -- & -- & -- & -- & \\
\hline EPW & 59 & 430220 & 710756 & GUYETTE & 1959 & 150 & -- & -- & -- & $\mathrm{x}$ & -- & \\
\hline EPW & 60 & 430223 & 710757 & DORSCH & 1966 & 150 & 48 & 22.0 & $\mathrm{R}$ & 0 & -- & \\
\hline
\end{tabular}




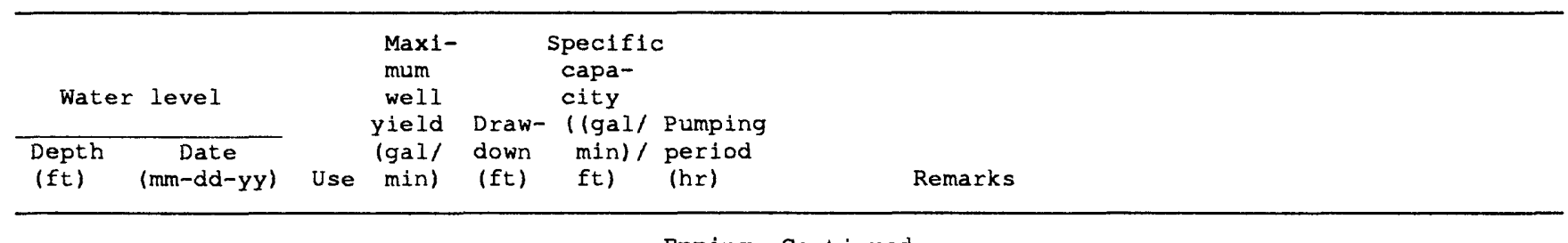

Epping--Cont inued

\begin{tabular}{|c|c|c|c|c|c|c|c|}
\hline-- & $=-$ & 0 & 30 & 7.1 & 4.3 & 4.5 & \\
\hline 2.4 & $03-20-78$ & 0 & 60 & - & - & & \\
\hline -- & -- & $\mathrm{H}$ & 3 & -- & -- & -- & \\
\hline 3 & $07-00-77$ & $\mathrm{H}$ & - & -- & -- & -- & RECENTLY PUMPED WELL \\
\hline- & -- & $\mathrm{P}$ & 100 & -- & -- & -- & \\
\hline-- & -- & $\mathrm{H}$ & -- & -- & -- & -- & \\
\hline 2.5 & $05-00-78$ & $\mathrm{H}$ & -- & - & -- & -- & \\
\hline- & - & $\mathrm{H}$ & 4 & - & -- & -- & \\
\hline 25 & $11-04-65$ & $\mathrm{H}$ & 4 & - & -- & -- & \\
\hline-- & -- & $\mathrm{H}$ & 8 & -- & -- & -- & \\
\hline-- & $05-28-75$ & 0 & 10 & - & -- & -- & \\
\hline 5.5 & $08-10-78$ & 0 & 50 & -- & -- & -- & \\
\hline 6.1 & $08-02-78$ & 0 & 50 & -- & - & -- & \\
\hline 6 & $08-02-78$ & 0 & 75 & -- & -- & -- & \\
\hline-- & -- & 0 & 25 & -- & -- & - & \\
\hline-- & -- & $\mathrm{H}$ & 15 & -- & -- & -- & \\
\hline-- & -- & $\mathrm{H}$ & 10 & -- & -- & -- & \\
\hline-- & -- & $\mathrm{H}$ & 30 & -- & - & -- & \\
\hline 25 & $09-27-71$ & $\mathrm{H}$ & 15 & -- & -- & - & \\
\hline 10 & $10-05-67$ & H & 4 & -- & -- & -- & \\
\hline 10.5 & $06-14-75$ & 0 & 60 & -- & -- & 2 & \\
\hline 30 & $05-05-65$ & $\mathrm{H}$ & 15 & -- & -- & -- & \\
\hline 24 & $06-18-65$ & $\mathrm{H}$ & 20 & - & - & - & \\
\hline 1.2 & $02-27-80$ & 0 & 50 & - & - & -- & \\
\hline-- & -- & $\mathrm{H}$ & 4 & -- & -- & -- & \\
\hline-- & -- & $\mathrm{H}$ & 100 & -- & -- & -- & \\
\hline-- & -- & $\mathrm{H}$ & 20 & -- & -- & -- & \\
\hline- & -- & $\mathrm{H}$ & 4 & -- & -- & -- & \\
\hline-- & -- & $\mathrm{H}$ & 6 & -- & -- & -- & \\
\hline 20 & $09-27-65$ & $\mathrm{H}$ & 12 & -- & -- & -- & \\
\hline 35 & $09-29-65$ & $\mathrm{H}$ & 100 & -- & -- & -- & \\
\hline-- & - & $\mathrm{H}$ & 1.5 & -- & -- & - & \\
\hline 26 & $09-03-63$ & $\mathrm{H}$ & 20 & -- & -- & -- & \\
\hline-- & -- & $\mathrm{H}$ & 2.5 & -- & -- & - & \\
\hline-- & - & H & 25 & -- & -- & - & \\
\hline-- & -- & $\mathrm{H}$ & 50 & -- & -- & -- & \\
\hline-- & -- & $\mathrm{H}$ & 10 & -- & -- & -- & \\
\hline-- & - & $\mathrm{H}$ & 3 & -- & - & -- & \\
\hline 7 & $02-23-73$ & 0 & 2.3 & -- & -- & -- & \\
\hline 10.6 & $06-13-75$ & 0 & 60 & 15.7 & -- & 3 & \\
\hline 1 & $02-26-80$ & 0 & 5 & -- & -- & -- & RECENTLY PUMPED WELL \\
\hline 1.6 & $03-03-80$ & 0 & 50 & -- & - & -- & \\
\hline 6.3 & $12-18-79$ & 0 & 60 & -- & -- & -- & INTERACTION WITH EPW 2 \\
\hline 6.3 & $12-19-79$ & 0 & 60 & - & -- & -- & INTERACTION WITH EPW 2 \\
\hline 6.2 & $12-20-79$ & 0 & 60 & -- & -- & -- & INTERACTION WITH EPW 2 \\
\hline 5.8 & $01-03-80$ & 0 & 40 & -- & -- & -- & INTERACTION WITH EPW 2 \\
\hline 5.6 & $08-29-85$ & 0 & - & - & -- & -- & USGS, CA \\
\hline-- & -- & $\mathrm{H}$ & -- & - & -- & -- & \\
\hline 13 & $00-00-59$ & $\mathrm{H}$ & 6 & - & - & -- & \\
\hline-1 & -- & $\mathrm{H}$ & - & - & - & -- & \\
\hline
\end{tabular}




\begin{tabular}{|c|c|c|c|c|c|c|c|c|c|c|c|}
\hline & & & & & Altitude & Diam- & Depth to & & & $\begin{array}{l}\text { Depth to } \\
\text { bottom }\end{array}$ & \\
\hline $\begin{array}{l}\text { Local } \\
\text { site } \\
\text { numver }\end{array}$ & $\begin{array}{l}\text { Lati- } \\
\text { tude }\end{array}$ & $\begin{array}{l}\text { Long- } \\
\text { tude }\end{array}$ & Owner or user & $\begin{array}{l}\text { Year } \\
\text { comp- } \\
\text { leted }\end{array}$ & $\begin{array}{c}\text { above } \\
\text { sea } \\
\text { level } \\
\text { (ft) }\end{array}$ & $\begin{array}{l}\text { eter } \\
\text { of } \\
\text { well } \\
\text { (in.) }\end{array}$ & $\begin{array}{l}\text { bottom } \\
\text { of } \\
\text { casing } \\
\text { (ft) }\end{array}$ & $\begin{array}{c}\text { Casing } \\
\text { mate- } \\
\text { rial }\end{array}$ & $\begin{array}{l}\text { Type } \\
\text { of } \\
\text { fin- } \\
\text { ish }\end{array}$ & $\begin{array}{c}\text { of } \\
\text { open } \\
\text { section } \\
\text { (ft) }\end{array}$ & $\begin{array}{l}\text { Type } \\
\text { of } \\
\text { site }\end{array}$ \\
\hline
\end{tabular}

Epping--Continued

\begin{tabular}{|c|c|c|c|c|c|c|c|c|c|c|c|c|}
\hline EPW & 61 & 430221 & 710804 & SMART THERESA & 1949 & 155 & -- & -- & -- & -- & -- & Dug \\
\hline EPW & 62 & 430224 & 710805 & FENSTERMAKER G & 1971 & 165 & 6 & 20.0 & $S$ & -- & -- & $\mathrm{BrW}$ \\
\hline EPW & 63 & 430224 & 710811 & FENSTERMAKER G & 1979 & 170 & -- & -- & -- & -- & -- & BrW \\
\hline EPW & 64 & 430222 & 710808 & OBRIEN COLIEEN & 1981 & 170 & -- & -- & -- & -- & -- & Brw \\
\hline EPW & 65 & 430217 & 710819 & HICKS MARY & 1981 & 165 & -- & -- & -- & 0 & -- & BrW \\
\hline EPW & 66 & 430331 & 710243 & USCOE & 1978 & 120 & 3 & 41.0 & $\mathrm{P}$ & -- & 51.0 & -- \\
\hline EPW & 67 & 430214 & 710807 & CARRIGAN R & 1983 & 160 & -- & -- & -- & 0 & -- & BrW \\
\hline EPW & 68 & 430116 & 710901 & LADNY GAIL & 1984 & 170 & -- & -- & -- & 0 & -- & BrW \\
\hline EPW & 69 & 430117 & 710852 & PERRY JANE & 1982 & 165 & -- & -- & -- & -- & -- & $\mathrm{BrW}$ \\
\hline EPW & 70 & 430144 & 710513 & JEAN J & 1884 & 145 & 28 & 12.0 & $\mathrm{R}$ & 0 & -- & Dug \\
\hline EPW & 71 & 430124 & 710512 & -- & -- & 150 & -- & -- & -- & -- & -- & -- \\
\hline EPW & 72 & 430128 & 710515 & KING $\mathrm{F}$ & 1979 & 150 & -- & -- & -- & $x$ & -- & $\mathrm{BrW}$ \\
\hline EPW & 73 & 430151 & 710655 & ATKIN & 1945 & 170 & 24 & 12.0 & $T$ & -- & -- & Dug \\
\hline EPW & 74 & 430154 & 710702 & RATCLIEF & 1981 & 160 & -- & -- & -- & $\mathrm{T}$ & -- & Dvn \\
\hline EPW & 76 & 430152 & 710704 & -- & 1977 & 160 & 19 & 19.0 & C & 0 & -- & Dug \\
\hline EPW & 77 & 430203 & 710728 & BROWN LAURIE & 1930 & 165 & -- & -- & -- & -- & -- & - \\
\hline EPW & 78 & 430201 & 710726 & -- & 1975 & 170 & 6 & 24.0 & $S$ & $\mathrm{P}$ & -- & Dvn \\
\hline EPW & 79 & 430156 & 710723 & STACKPOLE A & 1983 & 170 & 2 & 26.0 & $S$ & $S$ & -- & Dvn \\
\hline EPW & 80 & 430153 & 710715 & HOGAN & 1981 & 165 & 6 & 80.0 & $\mathrm{~S}$ & $x$ & -- & BrW \\
\hline EPW & 81 & 430154 & 710712 & HERSON G & 1979 & 165 & -- & -- & -- & -- & -- & Dug \\
\hline EPW & 82 & 430154 & 710712 & Foss L & -- & 165 & 2 & 28.0 & G & $\mathrm{P}$ & -- & Dvn \\
\hline EPW & 85 & 430146 & 710706 & -- & 1984 & 160 & 2 & 19.4 & $\mathrm{P}$ & $\mathrm{P}$ & 21.4 & Bor \\
\hline EPW & 86 & 430201 & 710728 & -- & 1984 & 172 & 2 & 42.5 & $\mathrm{P}$ & $\mathrm{P}$ & 44.5 & Bor \\
\hline EPW & 87 & 430211 & 710738 & -- & 1984 & 172 & 2 & 36.4 & $\mathrm{P}$ & $P$ & 38.4 & Bor \\
\hline EPW & 89 & 430248 & 710136 & -- & 1985 & 132 & 2 & 28.7 & $\mathrm{P}$ & $\mathrm{P}$ & 31.2 & Bor \\
\hline EPW & 90 & 430409 & 710101 & -- & 1985 & 120 & 2 & 34.5 & $\mathrm{P}$ & $\mathrm{P}$ & 37.0 & Bor \\
\hline EPW & 91 & 430241 & 710128 & -- & 1985 & 128 & 2 & 29.5 & $\mathrm{P}$ & $\mathrm{P}$ & 32.0 & Bor \\
\hline EPW & 92 & 430212 & 710812 & SALANDAR BRIAN & 1983 & 170 & -- & -- & -- & 0 & -- & BrW \\
\hline EPW & 93 & 430140 & 710431 & MCDONALD CORP & 1984 & 150 & -- & -- & -- & $\mathrm{x}$ & -- & BrW \\
\hline EPW & 94 & 430130 & 710554 & STILKEY & 1984 & 150 & -- & -- & -- & $\mathrm{X}$ & -- & $\mathrm{BrW}$ \\
\hline EPW & 95 & 430143 & 710606 & SWAIN & 1984 & 180 & -- & 19.0 & -- & $\mathrm{x}$ & -- & $\mathrm{BrW}$ \\
\hline EPW & 96 & 430146 & 710603 & REMICK & 1984 & 180 & -- & $-\infty$ & -- & $\mathrm{x}$ & -- & $\mathrm{BrW}$ \\
\hline EPW & 98 & 430157 & 710720 & CANTWELL & 1984 & 170 & -- & -- & -- & -- & -- & -- \\
\hline EPW & 99 & 430158 & 710721 & WENZEL & 1984 & 170 & -- & -- & -- & -- & -- & -- \\
\hline EPW & 101 & 430230 & 710651 & TOWNSEND & 1984 & 180 & -- & -- & -- & $x$ & -- & BrW \\
\hline EPW & 102 & 430242 & 710521 & BROOKS & 1984 & 160 & -- & -- & -- & $x$ & -- & BrW \\
\hline EPW & 103 & 430223 & 710358 & TELLY'S PIZZA & 1984 & 140 & -- & -- & -- & $\mathrm{x}$ & -- & $\mathrm{BrW}$ \\
\hline EPW & 104 & 430237 & 710356 & CLOUTIER, B & 1985 & 150 & -- & -- & -- & $\mathrm{X}$ & -- & Brw \\
\hline EPW & 105 & 430252 & 710340 & CLOUTIER & 1985 & 160 & -- & -- & -- & $\mathrm{x}$ & -- & BrW \\
\hline EPW & 106 & 430245 & 710412 & LANGDON HOMES & 1984 & 180 & -- & $-\infty$ & -- & $\mathrm{X}$ & -- & $\mathrm{BrW}$ \\
\hline EPW & 107 & 430245 & 710415 & BAKER & 1984 & 180 & -- & -- & -- & $\mathrm{x}$ & -- & Brw \\
\hline EPW & 108 & 430313 & 710343 & LETOURNEAU & 1984 & 180 & -- & -- & -- & $\mathrm{X}$ & -- & Brw \\
\hline EPW & 109 & 430328 & 710303 & BERNIER & 1984 & 150 & -- & -- & -- & $S$ & -- & -- \\
\hline EPW & 110 & 430330 & 710303 & BERNIER & 1984 & 150 & -- & -- & -- & $S$ & -- & -- \\
\hline EPW & 111 & 430339 & 710216 & SEIGEI & 1984 & 140 & -- & -- & -- & $\mathrm{x}$ & -- & $B r W$ \\
\hline EPW & 112 & 430340 & 710210 & LANGDON HOMES & 1984 & 150 & -- & -- & -- & $\mathrm{X}$ & -- & BrW \\
\hline EPW & 113 & 430340 & 710205 & LOVELIEN & 1985 & 140 & -- & -- & -- & $\mathrm{x}$ & -- & BrW \\
\hline EPW & 114 & 430344 & 710052 & HATCH & 1984 & 120 & -- & -- & -- & $\mathrm{X}$ & -- & BrW \\
\hline EPW & 115 & 430357 & 710103 & MISHIO & 1984 & 120 & -- & -- & -- & $x$ & -- & $\mathrm{BrW}$ \\
\hline EPW & 116 & 430407 & 710054 & KOLES & 1985 & 100 & -- & -- & -- & $\mathrm{x}$ & -- & Brw \\
\hline
\end{tabular}




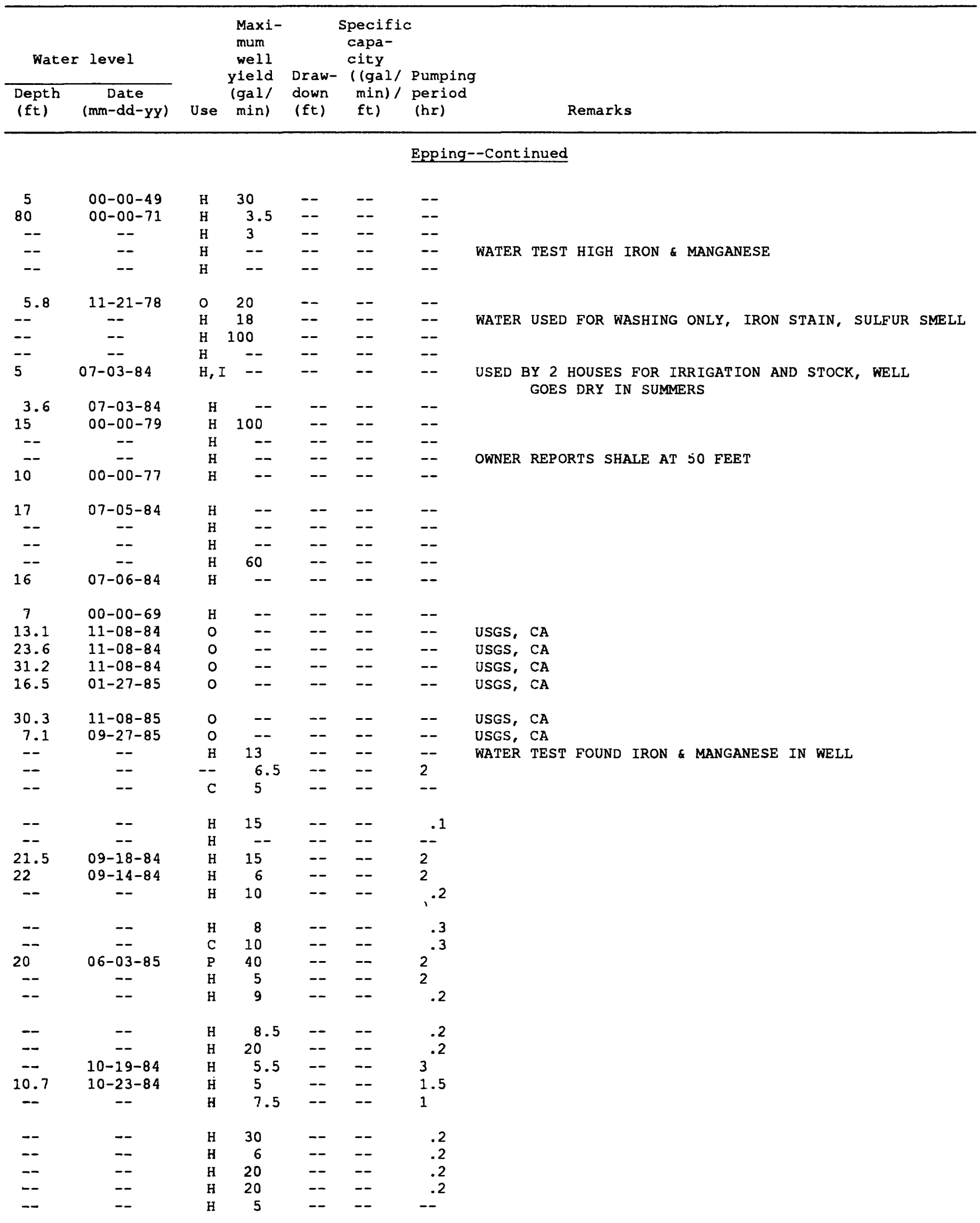




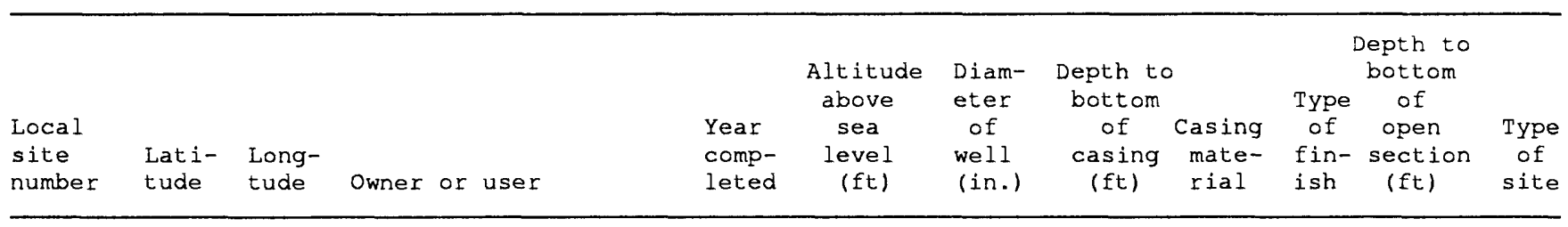

Epping--Cont inued

$\begin{array}{lllll}\text { EPW } & 117 & 430351 & 710340 & \text { LEE } \\ \text { EPW } & 118 & 430354 & 710343 & \text { INGRAHAM } \\ \text { EPW } & 119 & 430331 & 710717 & \text { PARKE } \\ \text { EPW } & 120 & 430201 & 710753 & \text { DAVIS } \\ \text { EPW } & 121 & 430200 & 710749 & \text { MURRAY } \\ \text { EPW } & 122 & 430158 & 710744 & \text { LOVELIEN }\end{array}$

EPW $122 \quad 430158 \quad 710744$

$\begin{array}{llllllll}1985 & 170 & -- & -- & -- & X & -- & \text { BrW } \\ 1984 & 170 & -- & -- & -- & X & -- & \text { BrW } \\ 1984 & 270 & -- & -- & -- & X & -- & \text { BrW } \\ 1984 & 160 & -- & -- & -- & X & -- & \text { BrW } \\ 1984 & 160 & -- & -- & -- & X & -- & \text { BrW } \\ 1984 & 160 & -- & -- & -- & X & -- & \text { BrW }\end{array}$

Exeter

\begin{tabular}{lrll} 
EXA & 1 & 430025 & 710028 \\
EXA & 2 & 430022 & 710029 \\
EXA & 3 & 430021 & 710028 \\
EXA & 4 & 430023 & 710028 \\
EXA & 5 & 430028 & 710029 \\
EXB & 2 & 425821 & 705705 \\
EXB & 3 & 425741 & 705723 \\
EXB & 4 & 425840 & 705427 \\
EXB & 6 & 430005 & 705651 \\
EXB & 7 & 430003 & 705639 \\
& & & \\
EXS & 1 & 425916 & 705725 \\
& & & \\
EXW & 2 & 425823 & 705640 \\
EXW & 3 & 430116 & 705824 \\
EXW & 4 & 430116 & 705929 \\
EXW & 5 & 430111 & 705921 \\
& & & \\
EXW & 6 & 430010 & 705848 \\
EXW & 7 & 430059 & 705628 \\
EXW & 8 & 425848 & 705738 \\
EXW & 9 & 425907 & 705757 \\
EXW & 10 & 425749 & 705925 \\
EXW & 11 & 425742 & 705959 \\
EXW & 12 & 425918 & 705950 \\
EXW & 13 & 425828 & 705620 \\
EXW & 14 & 425837 & 705510 \\
EXW & 15 & 425828 & 705628 \\
& & & \\
EXW & 17 & 425840 & 705630 \\
EXW & 18 & 425824 & 705620 \\
EXW & 19 & 425841 & 705433 \\
EXW & 20 & 425821 & 705424 \\
EXW & 21 & 425822 & 705503 \\
& & & \\
EXW & 22 & 425828 & 705425 \\
EXW & 23 & 425857 & 705604 \\
EXW & 24 & 425819 & 705558 \\
EXW & 25 & 425845 & 705618 \\
EXW & 26 & 425753 & 705712 \\
EXW & 27 & 425748 & 705711 \\
EXW & 28 & 425753 & 705647 \\
EXW & 41 & 425739 & 705609 \\
EXW & 42 & 425739 & 705606 \\
& & 425740 & 705557 \\
\hline
\end{tabular}

EXETER, TOWN OF EXETER, TOWN OF EXETER, TOWN OF EXETER, TOWN OF EXETER, TOWN OF

NHDOT

NHDOT

NHDOT

NHDOT

NHDOT

EXETER, TOWN OF

EXETER, TOWN OF

GOLD HARRY

WENTWORTH JOHN

WENTWORTH F L

CRONIN FRANK

HERRICK G C

EXETER BRASS WORKS

ELLISON ROBERT

GROVER J A

SIMPSON CHESTER

DOLLFF CARROLL

EXETER, TOWN OF

ROONEY FRED

EXETER, TOWN OF

EXETER, TOWN OF

EXETER, TOWN OF

EXETER, TOWN OF

EXETER, TOWN OF

EXETER, TOWN OF

EXETER, TOWN OF

EXETER, TOWN OF

EXETER, TOWN OF

EXETER, TOWN OF

EXETER, TOWN OF

EXETER, TOWN OF

EXETER, TOWN OF

EXETER, TOWN OF

EXETER, TOWN OF

EXETER, TOWN OF

$\begin{array}{rr}1982 & 100 \\ 1982 & 95 \\ 1982 & 100 \\ 1982 & 100 \\ 1982 & 100 \\ & \\ 1964 & 19 \\ 1934 & 30 \\ 1961 & 114 \\ 1958 & 81 \\ 1958 & 36 \\ & \\ -- & 30 \\ & \\ 1951 & 30 \\ -- & 150 \\ 1945 & 170 \\ 1928 & 170 \\ & \\ -- & 110 \\ 1955 & 30 \\ 1890 & 70 \\ 1939 & 70 \\ 1931 & 100 \\ 1945 & 150 \\ 1955 & 100 \\ 1955 & 30 \\ 1931 & 100 \\ 1956 & 30 \\ & \\ 1955 & 30 \\ 1955 & 30 \\ 1955 & 110 \\ 1955 & 80 \\ 1955 & 70 \\ & \\ 1955 & 100 \\ 1955 & 50 \\ 1955 & 30 \\ 1955 & 50 \\ 1955 & 40 \\ & \\ 1955 & 40 \\ 1955 & 40 \\ 1984 & 40 \\ 1984 & 40 \\ 1984 & 40\end{array}$

\begin{tabular}{|c|c|}
\hline-- & -- \\
\hline-- & -- \\
\hline-- & -- \\
\hline-- & -- \\
\hline-- & -- \\
\hline-- & -- \\
\hline-- & -- \\
\hline-- & -- \\
\hline-- & -- \\
\hline-- & -- \\
\hline-- & -- \\
\hline 24 & -- \\
\hline 36 & -- \\
\hline 36 & -- \\
\hline 6 & -- \\
\hline 36 & -- \\
\hline 48 & -- \\
\hline 8 & -- \\
\hline 6 & -- \\
\hline 6 & -- \\
\hline 6 & -- \\
\hline 24 & -- \\
\hline 2 & -- \\
\hline 24 & -- \\
\hline 8 & -- \\
\hline 2 & -- \\
\hline 2 & -- \\
\hline 2 & -- \\
\hline 2 & -- \\
\hline 2 & -- \\
\hline 2 & -- \\
\hline 2 & -- \\
\hline 2 & -- \\
\hline 2 & -- \\
\hline 2 & -- \\
\hline 2 & -- \\
\hline 2 & -- \\
\hline 3 & 100.0 \\
\hline 3 & 114.0 \\
\hline 2 & 11.5 \\
\hline
\end{tabular}

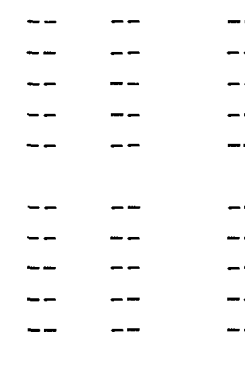

$\begin{array}{ll}-- & \text { Wsh } \\ -- & \text { Wsh } \\ -- & \text { Wsh } \\ -- & \text { Wsh } \\ -- & \text { Wsh } \\ & \\ -- & \text { Wsh } \\ -- & \text { Wsh } \\ -- & \text { Wsh } \\ -- & \text { Wsh } \\ -- & \text { Wsh }\end{array}$

- - - $\quad--\quad S p$

$\begin{array}{llll}\text { S } & -- & -- & \text { CbI } \\ \text { R } & -- & -- & \text { Dug } \\ \text { R } & -- & -- & \text { Dug } \\ \text { S } & -- & -- & \text { BrW } \\ & & & \\ \text { R } & -- & -- & \text { Dug } \\ \text { C } & -- & -- & \text { Dug } \\ \text { S } & -- & -- & \text { BrW } \\ \text { S } & -- & -- & \text { BrW } \\ \text { S } & -- & -- & \text { BrW }\end{array}$

$\begin{array}{llll}\text { S } & -- & -- & \text { BrW } \\ \text { T } & -- & -- & \text { Dug } \\ \text { S } & -- & -- & \text { Dvn } \\ \text { T } & -- & -- & \text { Dug } \\ \text { S } & -- & -- & \text { Cbl }\end{array}$

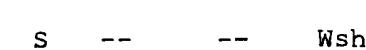

$\begin{array}{llll}\text { S } & -- & -- & \text { Wsh } \\ \text { S } & -- & -- & \text { Wsh } \\ \text { S } & -- & -- & \text { Wsh } \\ \text { S } & -- & -- & \text { Wsh }\end{array}$

S -- $\quad--\quad$ Wsh

s - = -- Wsh

$\begin{array}{llll}S & - & -- & \text { Wsh } \\ S & -- & -- & \text { Wsh } \\ \text { S } & -- & -- & \text { Wsh } \\ S & -- & -- & \text { Wsh }\end{array}$

$\begin{array}{llll}S & - & -- & \text { Wsh } \\ \mathrm{S} & -- & -- & \text { Wsh }\end{array}$

$s+\frac{10}{2-0}$

$\begin{array}{rrrr}\text { S } & -- & -- & \text { Wsh } \\ \text { S } & -- & -- & \text { Wsh } \\ \text { S } & \text { P } & 106.0 & \text { Wsh } \\ \text { S } & \text { P } & 120.0 & \text { Wsh } \\ \text { S } & \text { P } & 16.5 & \text { Wsh }\end{array}$




\begin{tabular}{|c|c|c|c|c|c|c|c|}
\hline Water & c level & & $\begin{array}{l}\text { Maxi- } \\
\text { mum } \\
\text { well } \\
\text { yield }\end{array}$ & Draw- & $\begin{array}{l}\text { Specific } \\
\text { capa- } \\
\text { city } \\
\text { (lgal) }\end{array}$ & Pumping & \\
\hline
\end{tabular}

$\begin{array}{rcccccc}-- & -- & \text { H } & 12 & -- & -- & 1 \\ -- & -- & \text { H } & 10 & -- & -- & .1 \\ 40 & 02-29-84 & \text { H } & 15 & -- & -- & 2 \\ 12 & 03-15-84 & \text { H } & 10 & -- & -- & - \\ 6 & 11-09-84 & \text { H } & 50 & -- & -- & .2\end{array}$

Exeter

\begin{tabular}{|c|c|c|c|c|c|c|}
\hline-- & -- & $\mathrm{T}$ & -- & -- & -- & - \\
\hline -- & -- & $T$ & -- & -- & -- & -- \\
\hline- & - & $T$ & $\cdots$ & -- & - & -- \\
\hline-- & -- & $T$ & -- & - & - & -- \\
\hline-- & - & $T$ & - & -- & -- & -- \\
\hline-- & $07-00-64$ & $T$ & -- & -- & - & - \\
\hline-- & -- & $T$ & -- & -- & - & -- \\
\hline- & - & $T$ & $\cdots$ & -- & - & -- \\
\hline-- & -- & $T$ & - & -- & -- & -- \\
\hline-- & -- & $T$ & -- & -- & -- & -- \\
\hline-- & -- & $\mathbf{P}$ & 6 & -- & -- & - \\
\hline 8.5 & $01-01-51$ & P & 500 & -- & - & - \\
\hline 14.5 & $08-05-55$ & 0 & -- & -- & -- & - \\
\hline 21.7 & $08-03-55$ & $\mathrm{H}$ & -- & -- & -- & -- \\
\hline-- & -- & $\mathrm{H}$ & 5.5 & -- & -- & -- \\
\hline 5.8 & $09-21-55$ & $\mathrm{U}$ & -- & -- & - & - \\
\hline-- & $05-08-56$ & $\mathrm{H}$ & -- & -- & -- & - \\
\hline$\ldots$ & -- & U & 10 & -- & -- & - \\
\hline 15 & $01-01-39$ & $\mathrm{H}$ & 13 & -- & -- & - \\
\hline 8.8 & $05-08-56$ & $\mathrm{H}$ & 5.5 & -- & -- & -- \\
\hline 28 & $01-01-45$ & $\mathrm{H}$ & 3.5 & -- & -- & $\cdots$ \\
\hline 2.4 & $05-17-56$ & $\mathrm{H}$ & - & -- & -- & - \\
\hline 11.2 & $05-18-56$ & 0 & $\cdots$ & -- & - & - \\
\hline 2.3 & $05-18-56$ & $\mathrm{U}$ & -- & -- & -- & - \\
\hline 11.5 & $11-13-56$ & 0 & 575 & -- & -- & - \\
\hline-- & -- & 0 & -- & -- & -- & $\ldots$ \\
\hline 9 & $01-01-55$ & 0 & 40 & -- & -- & -1 \\
\hline-- & -- & 0 & -- & -- & -- & - \\
\hline 9.4 & $01-01-55$ & 0 & 15 & -- & -- & - \\
\hline 4 & $01-01-55$ & 0 & 13 & -- & -- & -- \\
\hline 3.8 & $01-01-55$ & 0 & -- & -- & -- & - \\
\hline 7 & $01-01-55$ & 0 & -- & -- & $\cdots$ & $\cdots$ \\
\hline 6.8 & $01-01-55$ & 0 & -- & -- & -- & $\ldots$ \\
\hline-- & -- & 0 & -- & -- & -- & - \\
\hline-- & -- & 0 & -- & -- & -- & $\cdots$ \\
\hline-- & -- & 0 & -- & -- & -- & - \\
\hline-- & -- & 0 & -- & -- & -- & - \\
\hline 8.5 & $01-13-84$ & 0 & -- & -- & - & - \\
\hline 9 & $02-02-84$ & 0 & 60 & 2.5 & -- & .5 \\
\hline 1.6 & $03-27-84$ & 0 & -- & -- & -- & 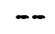 \\
\hline
\end{tabular}

WELL DRILLED IN FORMER DUG WELL

\section{Epping--Cont inued}

2

$+$

$-$

$-$

$-$

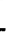

$-$

$-$

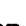

$-$

$-$

$-$

$-$

$-$

$-$

$-$

.50 


\begin{tabular}{|c|c|c|c|c|c|c|c|c|c|c|c|}
\hline $\begin{array}{l}\text { Local } \\
\text { site } \\
\text { number }\end{array}$ & $\begin{array}{l}\text { Lat 1- } \\
\text { tude }\end{array}$ & $\begin{array}{l}\text { Long- } \\
\text { tude }\end{array}$ & Owner or user & $\begin{array}{l}\text { Year } \\
\text { comp- } \\
\text { leted }\end{array}$ & $\begin{array}{l}\text { Altitude } \\
\text { above } \\
\text { sea } \\
\text { level } \\
\text { (ft) }\end{array}$ & $\begin{array}{l}\text { Diam- } \\
\text { eter } \\
\text { of } \\
\text { well } \\
\text { (in.) }\end{array}$ & $\begin{array}{l}\text { Depth to } \\
\text { bottom } \\
\text { of } \\
\text { casing } \\
\text { (ft) }\end{array}$ & $\begin{array}{l}\text { Casing } \\
\text { mate- } \\
\text { rlal }\end{array}$ & $\begin{array}{l}\text { Type } \\
\text { of } \\
\text { fin- } \\
\text { Ish }\end{array}$ & $\begin{array}{l}\text { Depth to } \\
\text { bottom } \\
\text { of } \\
\text { open } \\
\text { section } \\
\text { (ft) }\end{array}$ & $\begin{array}{l}\text { Type } \\
\text { of } \\
\text { site }\end{array}$ \\
\hline
\end{tabular}

Exeter--Cont inued

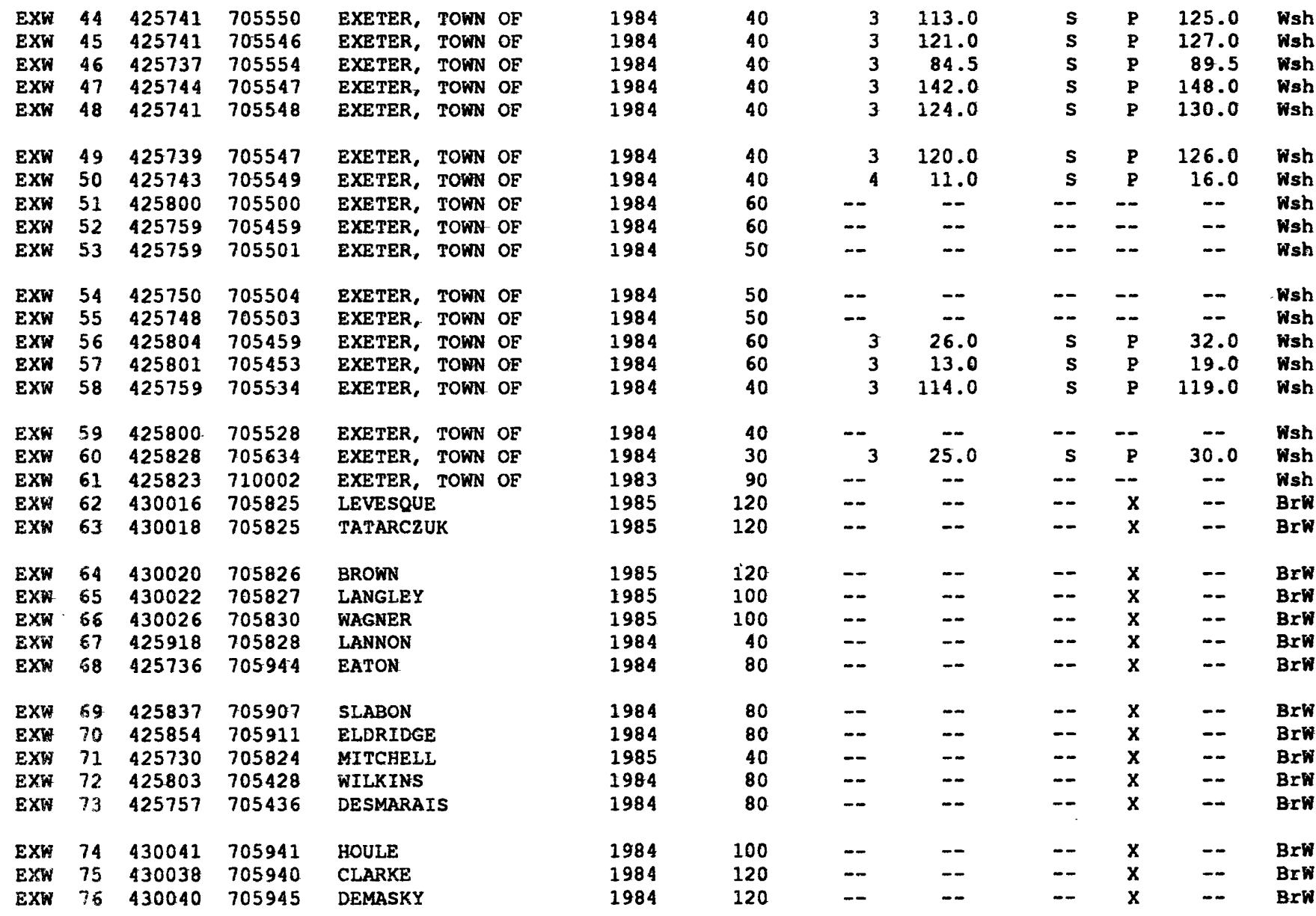

Fremont

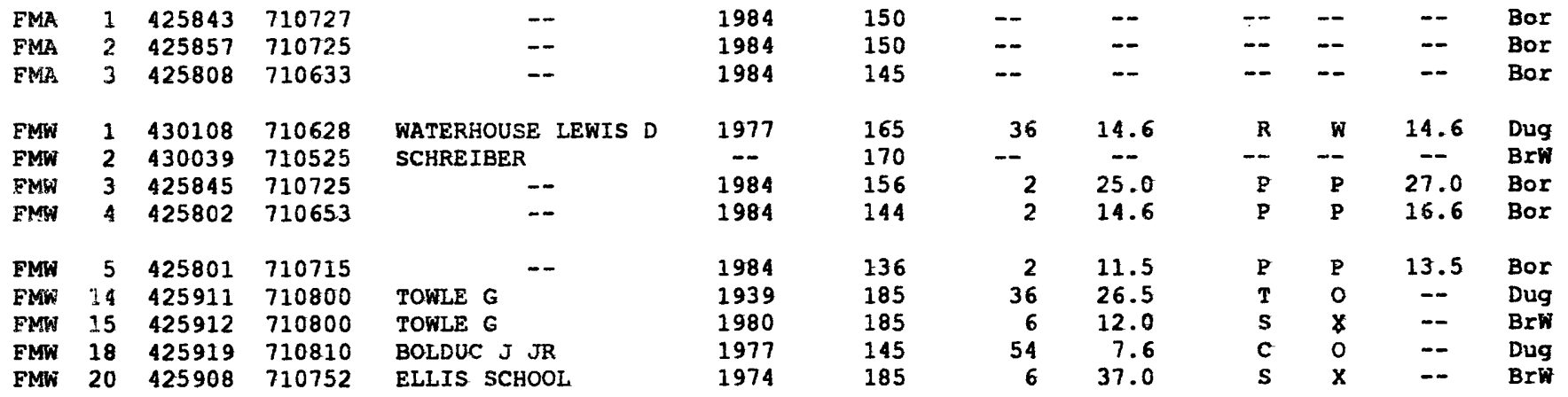




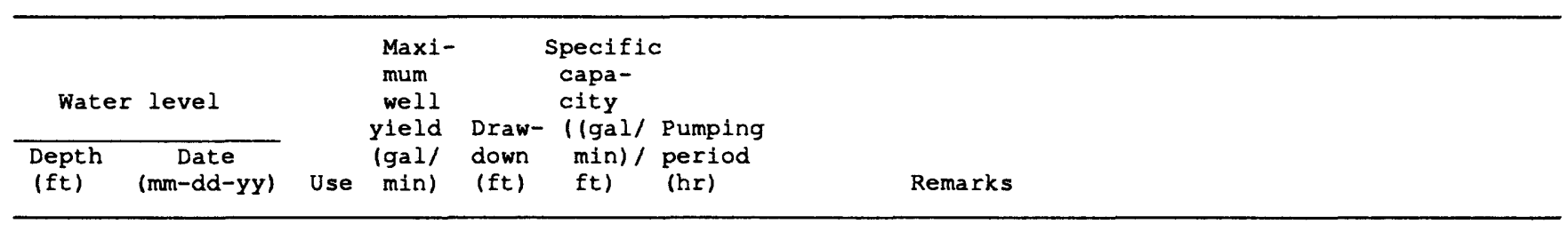

Exeter--Continued

\begin{tabular}{|c|c|c|c|c|c|c|}
\hline-- & -- & 0 & -- & -- & -- & -- \\
\hline 2.4 & $02-22-84$ & 0 & 55 & 3.1 & -- & 4.5 \\
\hline 6.5 & $03-08-84$ & 0 & -- & -- & -- & -- \\
\hline 2.3 & $03-19-84$ & 0 & -- & -- & -- & -- \\
\hline 3.3 & $03-23-84$ & 0 & 55 & 2.1 & -- & 2 \\
\hline 1.8 & $03-21-84$ & 0 & -- & -- & -- & -- \\
\hline 2.2 & $03-27-84$ & 0 & -- & -- & -- & -- \\
\hline- & -- & 0 & -- & -- & -- & -- \\
\hline-- & -- & 0 & -- & -- & -- & -- \\
\hline- & -- & 0 & -- & -- & -- & -- \\
\hline-- & -- & 0 & -- & -- & -- & -- \\
\hline-- & -- & 0 & -- & -- & -- & -- \\
\hline 5.6 & $01-20-84$ & 0 & -- & -- & -- & -- \\
\hline-- & -- & 0 & -- & -- & -- & -- \\
\hline 4.9 & $02-27-84$ & 0 & - & -- & -- & -- \\
\hline 2.5 & $03-01-84$ & 0 & -- & -- & -- & -- \\
\hline 2.3 & $03-23-84$ & 0 & 70 & -- & -- & 1.5 \\
\hline 24.5 & $03-04-83$ & 0 & -- & -- & -- & -- \\
\hline-- & -- & $\mathrm{H}$ & 20 & -- & -- & .5 \\
\hline-- & -- & $\mathrm{H}$ & 50 & -- & -- & .5 \\
\hline-- & -- & $\mathrm{H}$ & 2 & -- & -- & .5 \\
\hline-- & -- & $\mathrm{H}$ & 7 & -- & -- & .7 \\
\hline-- & -- & $\mathrm{H}$ & 50 & -- & -- & -- \\
\hline-- & -- & $\mathrm{H}$ & 5 & -- & -- & .2 \\
\hline- & -- & $\mathrm{H}$ & 30 & -- & -- & 1 \\
\hline-- & -- & $\mathrm{H}$ & 12 & -- & -- & .2 \\
\hline 2.5 & $04-04-84$ & $\mathrm{H}$ & 5.5 & -- & -- & 1 \\
\hline- & -- & $\mathrm{H}$ & 4.5 & -- & -- & -- \\
\hline-- & -- & $\mathrm{H}$ & 50 & -- & -- & -- \\
\hline-- & -- & $\mathrm{H}$ & 6 & -- & -- & -- \\
\hline-- & -- & $\mathrm{H}$ & 4.5 & -- & -- & .5 \\
\hline-- & - & $\mathrm{H}$ & 5 & - & -- & .5 \\
\hline -- & -- & $\mathrm{H}$ & 0.3 & -- & -- & -- \\
\hline
\end{tabular}

\section{Fremont}

$\begin{array}{llllllll}-- & -- & T & -- & -- & -- & -- & \text { USGS } \\ -- & -- & T & -- & -- & -- & -- & \text { USGS } \\ -- & -- & \text { T } & -- & -- & -- & -- & \text { USGS }\end{array}$

$\begin{array}{cccccccl}10.6 & 01-24-78 & 0 & -- & -- & -- & -- & \\ -- & -- & \text { H } & -- & -- & -- & -- & \text { USGS, CA } \\ 15.1 & 11-09-84 & 0 & -- & -- & -- & -- & \text { USGS, CA } \\ 8.9 & 11-09-84 & 0 & -- & -- & -- & -- & \\ 9.9 & 11-09-84 & 0 & -- & -- & -- & -- & \text { USGS, CA } \\ -- & -- & \text { H } & -- & -- & -- & -- & \text { WATER USED FOR WASHING ONLY } \\ 22 & 06-12-84 & \text { H } & 3 & -- & -- & -- & \text { WATER TESTED BY WSPCC, SOFT } \\ 3 & 06-17-84 & \text { H } & -- & -- & -- & -- & \\ -- & -- & \text { T } & -- & -- & -- & -- & \text { WATER TESTED BY WSPCC, GOOD, SLIGHT IRON STAIN }\end{array}$




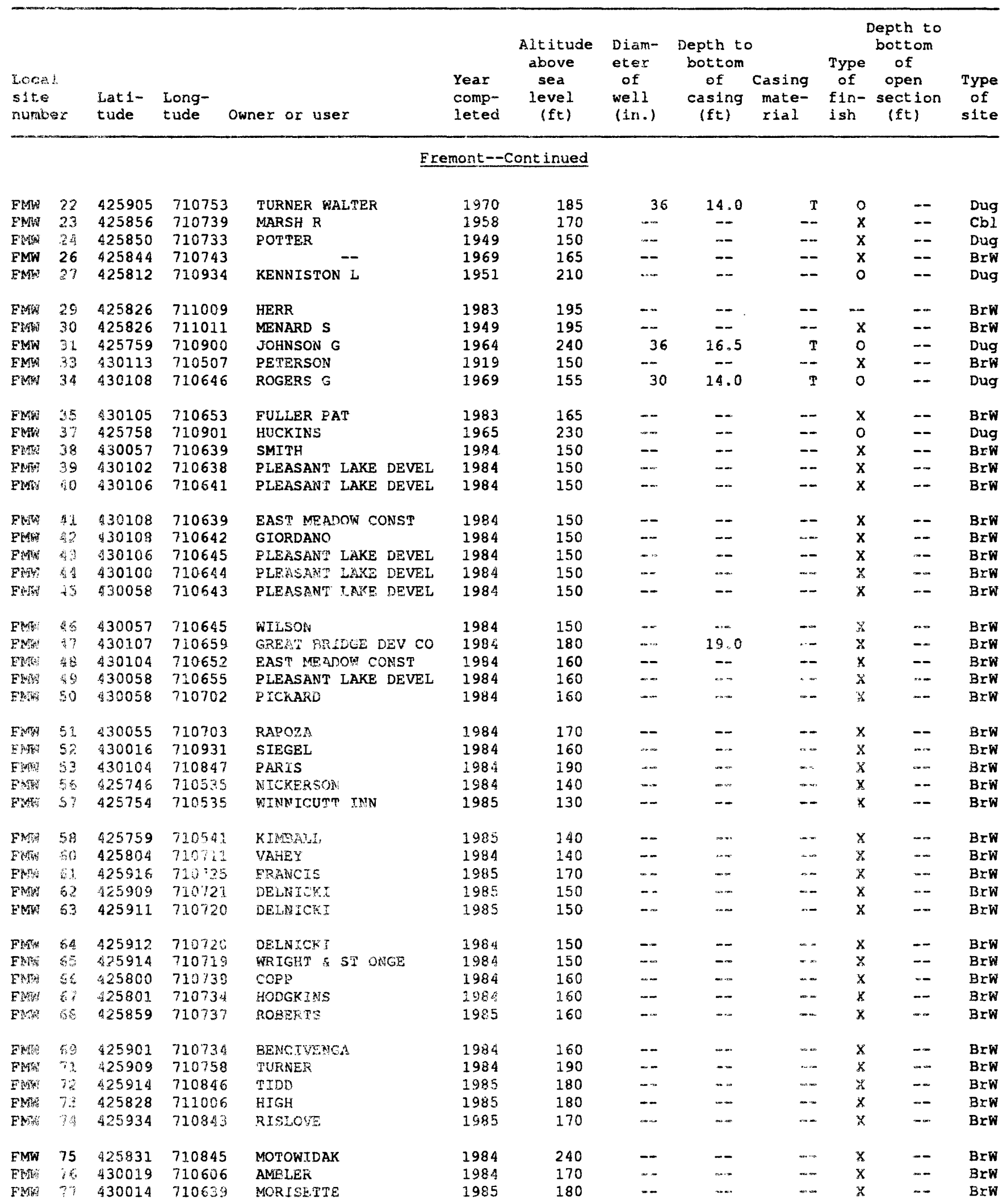




\section{and springs--Continued}

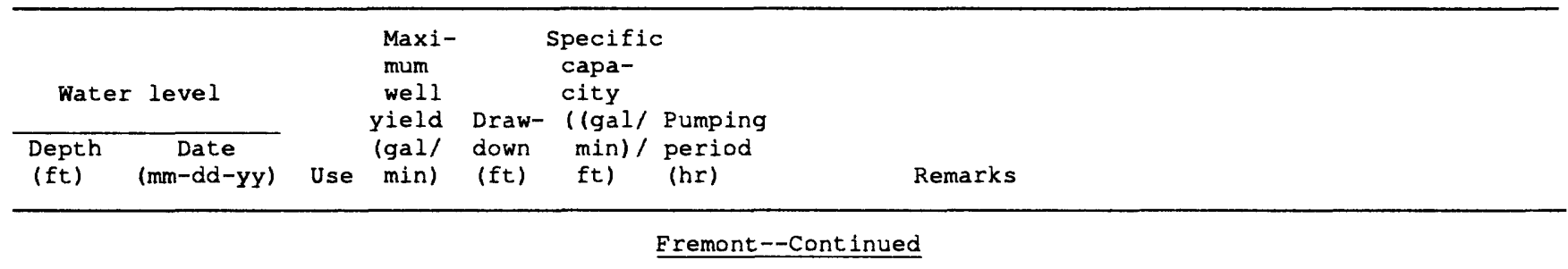

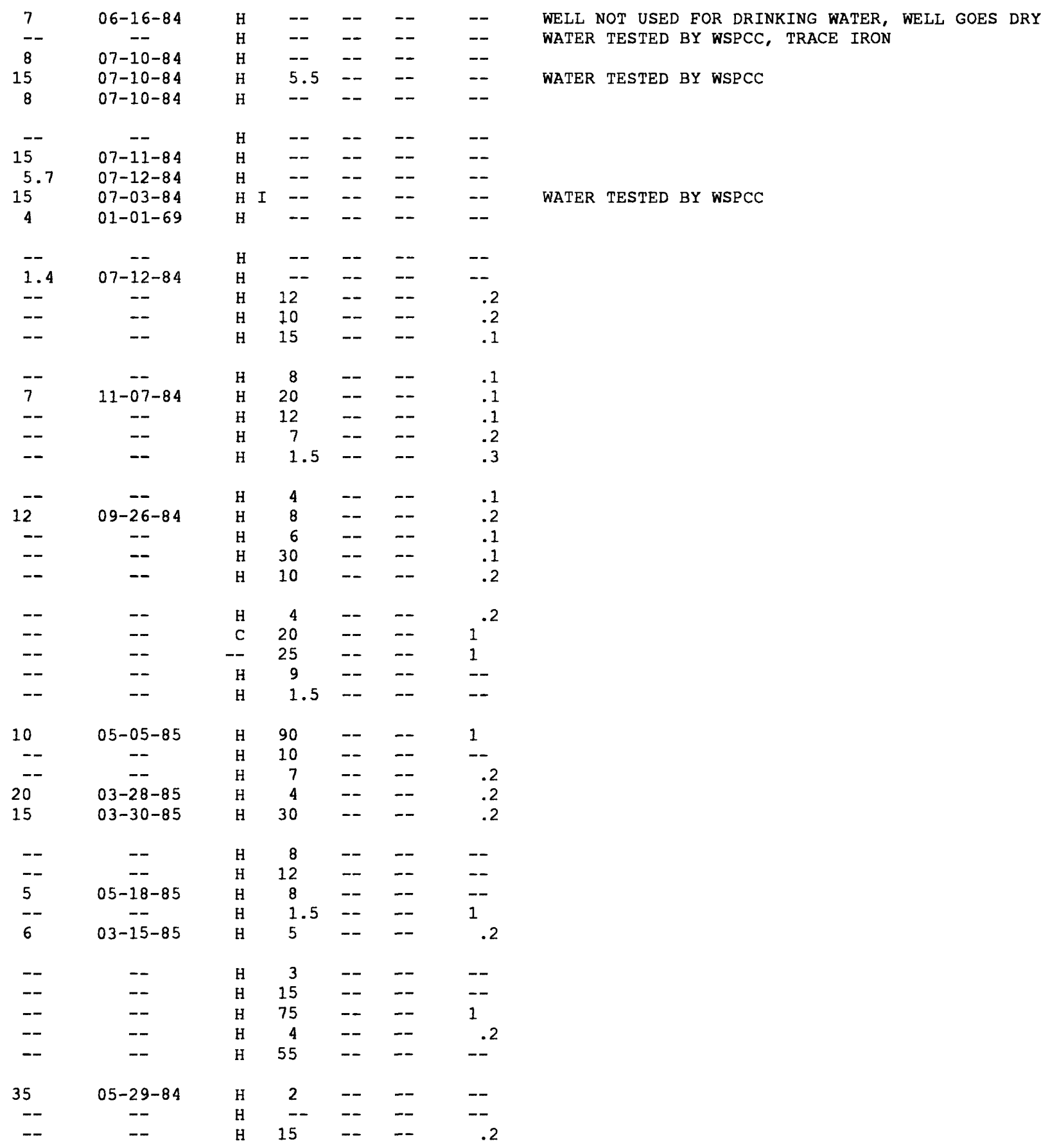




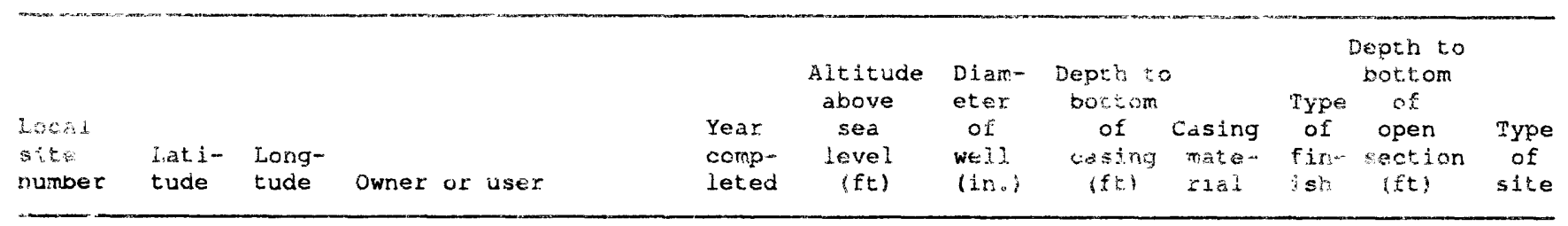

Hampstead

\begin{tabular}{|c|c|c|c|c|c|c|c|c|c|c|c|c|}
\hline HLW & 2 & 425439 & 711259 & -- & -- & 410 & 24 & $m$ & $\cdots$ & $-\infty$ & $\cdots$ & Dug \\
\hline HCt & 29 & 425322 & 711006 & ORDWAY, MYSON & $\cdots$ & 255 & $\cdots$ & $\ldots$ & $-\infty$ & $\infty$ & $\ldots$ & BrW \\
\hline HDt & 30 & 425319 & 711013 & REGAN, MIRRAN & -- & 235 & - & 98.0 & -- & -- & - & $B r W$ \\
\hline How & 72 & 425345 & 711251 & BURGER & 1984 & 300 & -- & -- & -- & $x$ & - & BrW \\
\hline HDN & 32 & 425326 & 711215 & $\operatorname{CCLSIA}$ & 1985 & 260 & $=\infty$ & $\ldots$ & $-\infty$ & $x$ & $=-$ & $8 . W$ \\
\hline HUN & 33 & 425323 & 711216 & CZOUTIER LONST & 1985 & 260 & - & -- & -- & $x$ & - & $\mathrm{BrW}$ \\
\hline ERST & 34 & 425322 & 711212 & SLOUTERR CONST & 1985 & 250 & $-\infty$ & -- & $-\cdots$ & $x$ & $-\infty$ & BrW \\
\hline HDW & 25 & 425326 & 711210 & FARRET.T. & 1986 & 250 & $\rightarrow \infty$ & $-\infty$ & $=-$ & * & $-\infty$ & $B \times W$ \\
\hline How & 36 & 425328 & 711208 & FARKELL & 1986 & 250 & $-\infty$ & $\cdots$ & $=-t a$ & $x$ & 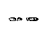 & $B r W$ \\
\hline HDT & 3 & $\$ 25330$ & 711209 & REDDY HOME BLDRS & 1986 & 250 & $x=2$ & $\ldots$ & $=-$ & g & $\infty$ & $B E N$ \\
\hline HDW & 38 & 425425 & 711130 & $B \& B$ BI.DRS & 1985 & 250 & $\cdots$ & -- & $-\infty$ & $\mathrm{x}$ & $-\infty$ & Brw \\
\hline How & 39 & 425342 & 711115 & HOUDE & 1985 & 320 & $\infty$ & $x$ & ${ }_{x=}=$ & $x$ & $=-\infty$ & BrW \\
\hline $\mathrm{HDS}$ & 40 & 425332 & 711116 & ASHFORD & 1584 & 300 & $=\infty$ & $\rightarrow$ & 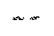 & k & $=-$ & Brw \\
\hline HEN & 41 & 425330 & 711114 & ASUPCPD & 1984 & 300 & -- & $-\cdots$ & $-\infty$ & $x$ & $-\infty$ & BrW \\
\hline HDW & 42 & 425332 & 711109 & RENAUD & 1984 & 300 & $\ldots$ & $\therefore$ & $-\infty$ & $x$ & $-\infty$ & Bring \\
\hline $10 w$ & $\because$ & 425351 & 711049 & HOWARD & 1985 & 330 & $\infty+\infty$ & -- & $\infty$ & $x$ & $-\infty$ & Bry \\
\hline $\mathrm{xn}$ & 135 & 425352 & 711259 & LETSON & 1985 & 270 & -- & $\Leftrightarrow$ & $-\infty$ & $X$ & - & Brw \\
\hline How & 116 & 425348 & 711255 & WILSON & 1984 & 280 & $a$ & $=\ldots$ & $\infty-$ & 3 & $x-$ & SrW \\
\hline
\end{tabular}

Hampton Falls

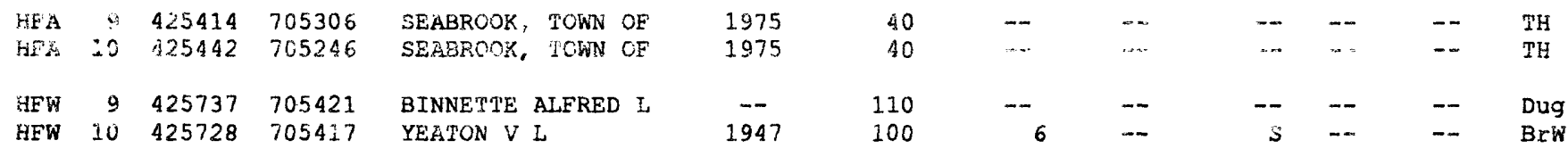

Kensington

$\begin{array}{llll}\text { KFS } & 1 & 425705 & 705819 \\ & & & \\ \text { KFW } & 1 & 425623 & 705744 \\ \text { KFW } & 2 & 425502 & 705836 \\ \text { KFW } & 3 & 425644 & 705559 \\ \text { KFW } & 4 & 425614 & 705655 \\ & & & \\ \text { KFW } & 5 & 425622 & 705757 \\ \text { KFW } & 6 & 425623 & 705817 \\ \text { KFW } & 7 & 425544 & 705636 \\ \text { KFW } & 8 & 425527 & 705618 \\ \text { KFW } & 9 & 425534 & 705735\end{array}$

KIMBALL MRS FRANK

SWIET GORDON MONAHAN BETSY $J$ YORK $I$ W

TOOTHACRE F E

MATHEWS CHARLES

HUTCHINSON C R KENSINGTON SCHOOL BRAGG ALICE E

GROVE AMOS S

$\begin{array}{cr}-- & 40 \\ 1953 & 210 \\ -- & 270 \\ 1953 & 80 \\ 1926 & 110 \\ 1954 & 220 \\ 1952 & 250 \\ 1952 & 130 \\ 1931 & 130 \\ -- & 230 \\ & \end{array}$

\section{Kingston}

KINGSTON, TOWN OF D'URSO, BOB

1956

1986

1956 KINGSTON, TOWN OF KINGSTON, TOWN OF

1985

120
110
120
140
135
140
240
135

BAKIE, WARREN G JAMESON CHARLES F SIMPSON CLAYTON W

$\begin{array}{cl}-- & 135 \\ -- & 140 \\ 1935 & 240 \\ -- & 135\end{array}$

$\begin{array}{rr}-- & - \\ -- & -- \\ -- & -- \\ -- & -- \\ 36 & -- \\ 42 & -- \\ 6 & - \\ 120 & --\end{array}$

$\mathrm{Sp}$

Dug

Dug

Brn BrW

BrW

BrW

CbI

BrW

Dug 


\begin{tabular}{|c|c|c|c|c|c|c|c|}
\hline Wate & level & & $\begin{array}{l}\text { Maxi- } \\
\text { mum } \\
\text { well }\end{array}$ & $\mathbf{S}$ & $\begin{array}{l}\text { Specific } \\
\text { capa- } \\
\text { city }\end{array}$ & & \\
\hline
\end{tabular}

\section{Hampstead}

$\begin{array}{rrrrrrr}21.6 & 08-21-62 & \text { H } & -- & -- & -- & -- \\ 23 & --00 & \text { H } & 4.5 & -- & -- & -- \\ 25 & --00 & \text { H } & 9 & -- & -- & -- \\ 25 & 09-28-84 & \text { H } & 5 & -- & -- & 4 \\ & & & & & & \\ 20 & 08-15-85 & \text { H } & 4 & -- & -- & .5 \\ 6 & 12-05-85 & \text { H } & 15 & -- & -- & .5 \\ 10 & 12-06-85 & \text { H } & 60 & -- & -- & .5 \\ 20 & 02-14-86 & \text { H } & 3 & -- & -- & .5 \\ 20 & 02-17-86 & \text { H } & 8 & -- & -- & .5 \\ & & & & & & \\ 20 & 02-14-86 & \text { H } & 8 & -- & -- & .5 \\ -- & -- & \text { H } & 1.5 & -- & -- & 1 \\ 15 & 06-01-85 & \text { H } & 2.5 & -- & -- & 5 \\ 25 & 05-17-84 & \text { H } & 5 & -- & -- & 4 \\ 25 & 05-18-84 & \text { H } & 5 & -- & -- & 4 \\ & & & & & & \\ 28 & 08-15-84 & \text { H } & 5 & -- & -- & 4 \\ 20 & 06-22-85 & \text { H } & 4 & -- & -- & 4 \\ 20 & 04-02-85 & \text { H } & 4 & -- & -- & .5 \\ 30 & 10-07-84 & \text { H } & 5 & -- & -- & 4\end{array}$

\section{Hampton Falls}

$\begin{array}{rllllll}2.5 & 04-23-75 & \text { T } & -- & -- & -- & -- \\ 1.7 & 04-10-75 & \text { T } & -- & -- & -- & -- \\ 5.6 & 04-19-56 & \text { H } & -- & -- & -- & -- \\ 19 & 01-01-47 & \text { H } & -- & -- & -- & --\end{array}$

Kensington

$\begin{array}{ccccccc}-- & -- & \text { H } & 11 & -- & -- & -- \\ 9.6 & 07-13-54 & \text { U } & -- & -- & -- & -- \\ 10.5 & 07-13-54 & \text { U } & -- & -- & -- & -- \\ -- & -- & \text { H } & 6 & -- & -- & -- \\ 22 & 01-01-26 & \text { H } & 2.5 & -- & -- & -- \\ 28 & 01-01-54 & \text { H } & 5 & -- & -- & -- \\ 30 & 01-01-52 & \text { H } & 20 & -- & -- & -- \\ 17 & 01-01-52 & \text { T } & 30 & -- & -- & -- \\ -- & -- & \text { H } & 20 & -- & -- & -- \\ 7.5 & 05-21-56 & \text { H } & -- & -- & -- & -\end{array}$

\section{Kingston}

$\begin{array}{lclllll}-- & -- & \text { T } & -- & -- & -- & -- \\ 6 & 08-05-86 & \text { T } & -- & -- & -- & -- \\ -- & -- & \text { T } & -- & -- & -- & -- \\ -- & -- & \text { T } & -- & -- & -- & -- \\ 7.5 & 12-22-55 & \text { H } & -- & -- & -- & -- \\ 5.4 & 12-22-55 & \text { H } & -- & -- & -- & -- \\ -- & -- & \text { H } & 7 & -- & -- & -- \\ 7.1 & 06-18-56 & \text { P } & -- & -- & -- & --\end{array}$


Table 2.--Description of wells, borings,

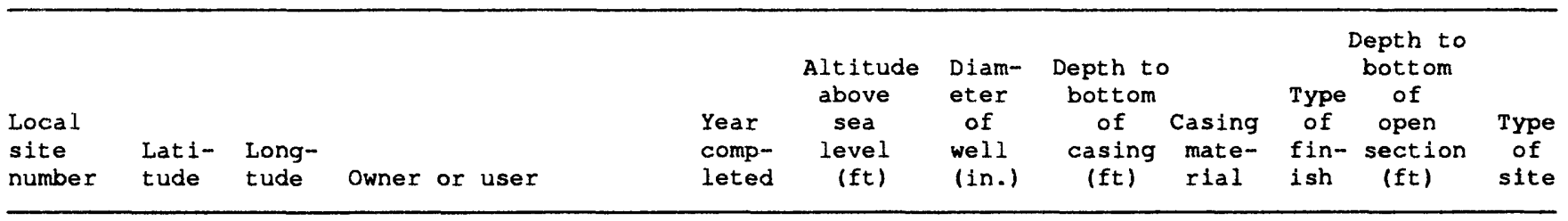

Kingston--Continued

$\begin{array}{llllll}\text { KTW } & 13 & 425628 & 710436 & \text { RICHARDSON F } & \text { A } \\ \text { KTW } & 19 & 425636 & 710338 & \\ \text { KTW } & 30 & 425607 & 710428 & \text { MEYER } & \\ \text { KTW } & 31 & 425607 & 710456 & \text { DONAHEAU } \\ \text { KTW } & 32 & 425712 & 710156 & \text { DECARLO } \\ & & & & \\ \text { KTW } & 33 & 425714 & 710154 & \text { PLANTE } \\ \text { KTW } & 34 & 425727 & 710155 & \text { ST PIERRE } \\ \text { KTW } & 35 & 425708 & 710041 & \text { EGAN } \\ \text { KTW } & 36 & 425717 & 710035 & \text { GOODWIN } \\ \text { KTW } & 37 & 425729 & 710031 & \text { CYNEWSKI } \\ & & & & \\ \text { KTW } & 38 & 425729 & 710039 & \text { COTE } \\ \text { KTW } & 43 & 425717 & 710420 & \text { VERDA }\end{array}$

$\begin{array}{cc}1985 & 150 \\ 1984 & 140 \\ 1984 & 170 \\ -- & 200 \\ 1985 & 105 \\ 1985 & 100 \\ 1985 & 140 \\ 1985 & 150 \\ 1985 & 160 \\ 1985 & 250 \\ 1984 & 230 \\ & 140\end{array}$

\begin{tabular}{|c|c|c|c|c|c|}
\hline 36 & -- & $\mathbf{R}$ & -- & -- & Dug \\
\hline 2 & 52.5 & P & P & 55.0 & Bor \\
\hline-- & -- & - & $x$ & -- & BrW \\
\hline-- & -- & - & $x$ & -- & $\mathrm{BrW}$ \\
\hline-- & -- & - & $x$ & $-\infty$ & BrW \\
\hline- & $-\cdots$ & $-\cdots$ & $x$ & -- & Brw \\
\hline-- & -- & - & $x$ & $-\infty$ & Brw \\
\hline-- & -- & - & $\mathbf{x}$ & -- & BrW \\
\hline-- & -- & - & $x$ & $-\infty$ & BrW \\
\hline - & - & חנ - & $x$ & -- & Brw \\
\hline-- & - & $-\ldots$ & $x$ & - & Brw \\
\hline-- & - & - & $x$ & -- & $\mathrm{Br}$ \\
\hline
\end{tabular}

Newfields

$\begin{array}{lrll}\text { NGA } & 1 & 430205 & 705951 \\ \text { NGS } & 1 & 430247 & 706000 \\ & & & \\ \text { NGW } & 1 & 430221 & 705615 \\ \text { NGW } & 2 & 430227 & 710033 \\ \text { NGW } & 3 & 430245 & 705803 \\ \text { NGW } & 4 & 430215 & 705625 \\ & & & \\ \text { NGW } & 5 & 430214 & 705628 \\ \text { NGW } & 6 & 430220 & 705608 \\ \text { NGW } & 7 & 430223 & 705615 \\ \text { NGW } & 8 & 430223 & 705615 \\ \text { NGW } & 12 & 430304 & 710012 \\ & & & \\ \text { NGW } & 13 & 430239 & 710009 \\ \text { NGW } & 14 & 430155 & 705753 \\ \text { NGW } & 15 & 430119 & 705636 \\ \text { NGW } & 16 & 430225 & 705607 \\ \text { NGW } & 17 & 430244 & 705533\end{array}$

$\begin{array}{lrll}\text { NMA } & 1 & 430447 & 705829 \\ \text { NMA } & 2 & 430446 & 705856 \\ \text { NMA } & 3 & 430457 & 705753 \\ \text { NMA } & 4 & 430451 & 705857 \\ \text { NMA } & 5 & 430501 & 705642 \\ \text { NMA } & 6 & 430325 & 705639 \\ \text { NMA } & 7 & 430321 & 705633 \\ \text { NMA } & 8 & 430328 & 705613 \\ \text { NMA } & 10 & 430340 & 705759 \\ \text { NMA } & 11 & 430446 & 705447 \\ & & & \\ \text { NMA } & 12 & 430332 & 705759 \\ \text { NMA } & 13 & 430307 & 705658 \\ \text { NMA } & 14 & 430321 & 705959 \\ \text { NMA } & 15 & 430325 & 705958 \\ \text { NMA } & 16 & 430341 & 710000\end{array}$

NEWMARKET, TOWN OF NEWMARKET, TOWN OF NEWMARKET, TOWN OF NEWMARKET, TOWN OF NEWMARKET, TOWN OF NEWMARKET, TOWN OF NEWMARKET, TOWN OF NEWMARKET, TOWN OF NEWMARKET, TOWN OF NEWMARKET, TOWN OF

NEWMARKET, TOWN OF NEWMARKET, TOWN OF NEWMARKET, TOWN OF NEWMARKET, TOWN OF NEWMARKET, TOWN OF

1985
--
1954
1950
--
--
1945
1945
1966
1977
1985
1985
1984
1985
1985
1984

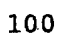

100

50

120

140

100

110

40
40

40

132

100

120

60
.20

10
Newmarket

$\begin{array}{rr}1964 & 130 \\ 1964 & 140 \\ 1964 & 100 \\ 1964 & 110 \\ 1965 & 50 \\ 1980 & 80 \\ 1980 & 80 \\ 1980 & 120 \\ 1980 & 80 \\ 1980 & 30 \\ & \\ 1980 & 90 \\ 1980 & 90 \\ 1981 & 100 \\ 1981 & 100 \\ 1981 & 115\end{array}$




\section{and springs--Continued}

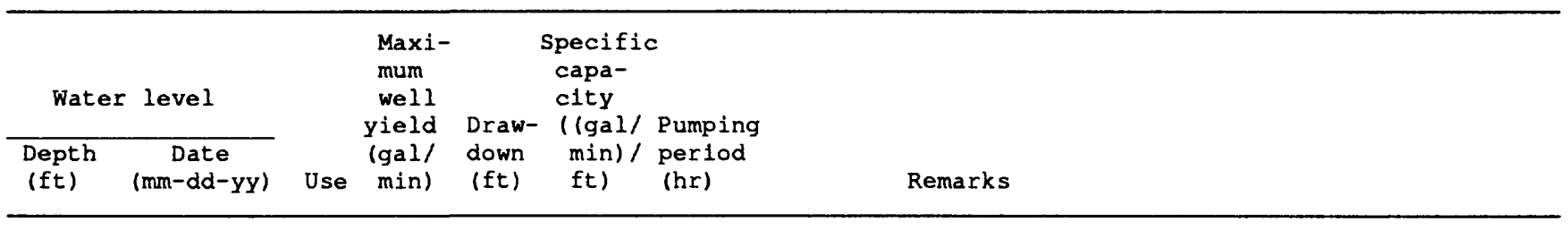

Kingston--Cont Inued

$\begin{array}{cccccccc}9.7 & 08-09-56 & \text { H } & -- & -- & -- & -- & \\ 16.9 & 09-30-85, & \text { O } & -- & -- & -- & -- & \text { USGS, CA } \\ -- & -- & \text { H } & 10 & -- & -- & .1 & \\ 5 & 09-05-84 & \text { H } & 6 & -- & -- & 1 & \\ -- & -- & -- & 7 & -- & -- & 1 \\ -- & -- & \text { H } & 10 & -- & -- & -- \\ 15 & 04-07-85 & \text { H } & 2.5 & -- & -- & 1 \\ -- & -- & \text { H } & 3 & -- & -- & 1.7 \\ -- & -- & \text { H } & 5 & -- & -- & .7 \\ -- & -- & \text { H } & 15 & -- & -- & --\end{array}$

$\begin{array}{lllllll}30 & 04-05-85 & \mathrm{H} & 5 & -- & -- & 4 \\ 10 & 06-20-84 & \mathrm{H} & 5 & -- & -- & \end{array}$

\section{Newfields}

$\begin{array}{ccccccccc}-- & -- & \text { T } & -- & -- & -- & -- & \text { USGS } \\ -- & -- & \text { H } & 12 & -- & -- & -- & \\ 17 & 01-01-54 & \text { P } & 45 & -- & -- & -- & \\ 4.6 & 07-21-55 & \text { H } & -- & -- & -- & -- & \\ 12.8 & 07-20-55 & \text { U } & -- & -- & -- & -- & \\ 39.9 & 09-13-55 & \text { U } & -- & -- & -- & -- & \\ 34 & 01-01-45 & \text { H } & -- & -- & -- & -- & \\ 14 & 01-01-45 & \text { H } & 10 & -- & -- & -- & & \\ -- & -- & \text { P } & -- & -- & -- & -- & & \\ -- & -- & \text { P } & -- & -- & -- & -- & \\ 31.7 & 09-27-85 & \text { O } & -- & -- & -- & -- & \text { USGS, CA } \\ -- & 09-20-85 & \text { O } & -- & -- & -- & -- & \text { USGS, FLOWING WELL, CA } \\ 10 & 12-15-84 & \text { H } & 2.5 & -- & -- & 1 & & \\ 15 & 05-23-85 & \text { H } & 60 & -- & -- & -5 & \\ -- & -- & \text { H } & 20 & -- & -- & -- & \\ -- & -- & \text { H } & 4 & -- & -- & 1 & \end{array}$

Newmarket

\begin{tabular}{|c|c|c|c|c|c|}
\hline-- & -- & $T$ & -- & -- & -- \\
\hline-- & -- & $\mathbf{T}$ & -- & -- & -- \\
\hline-- & -- & $\mathrm{T}$ & -- & -- & -- \\
\hline-- & -- & $\mathrm{T}$ & -- & -- & -- \\
\hline-- & -- & $\mathrm{T}$ & -- & -- & -- \\
\hline-- & -- & $T$ & -- & -- & -- \\
\hline-- & -- & $T$ & -- & -- & -- \\
\hline-- & -- & $T$ & -- & -- & -- \\
\hline-- & -- & $T$ & -- & -- & -- \\
\hline-- & -- & $T$ & -- & -- & -- \\
\hline-- & -- & $T$ & -- & -- & -- \\
\hline-- & -- & $T$ & -- & -- & -- \\
\hline-- & -- & $T$ & -- & -- & -- \\
\hline-- & -- & $T$ & -- & -- & -- \\
\hline-- & -- & $T$ & -- & -- & -- \\
\hline
\end{tabular}




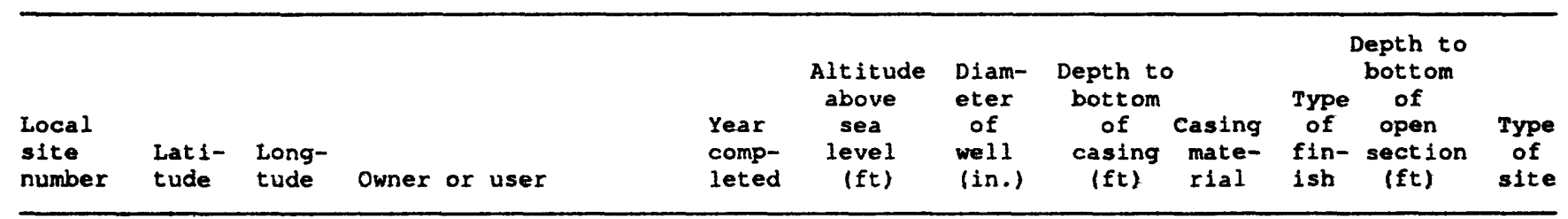

Newmarket --Cont inued

\begin{tabular}{|c|c|c|c|c|c|c|c|c|c|c|c|c|c|c|}
\hline NMA & 17 & 430346 & 710000 & NEWMARKET, & TOWN & OF & 1981 & 115 & -- & -- & -- & -- & -- & TH \\
\hline NMA & 18 & 430343 & 705957 & NEWMARKET, & TOWN & OF & 1981 & 130 & -- & -- & -- & -- & -- & TH \\
\hline NMA & 19 & 430338 & 705927 & NEWMARKET, & TOWN & OF & 1981 & 160 & -- & -- & -- & -- & -- & TH \\
\hline NMA & 20 & 430338 & 705936 & NEWMARKET, & TOWN & OF & 1981 & 150 & -- & -- & -- & - & -- & TH \\
\hline NMA & 22 & 430507 & 705715 & NEMMARKET, & TOWN & OF & 1981 & 60 & -- & -- & -- & -- & -- & TH \\
\hline NMB & 1 & 430336 & 705800 & NHDOT & & & 1959 & 70 & -- & -- & -- & -- & -- & พิsh \\
\hline NMS & 1 & 430335 & 705943 & \multirow{2}{*}{\multicolumn{3}{|c|}{ HILTON LYDIA }} & -- & 120 & -- & -- & -- & -- & -- & Sp \\
\hline NMS & 2 & 430341 & 705604 & & & & -- & 60 & -- & -- & -- & -- & -- & Sp \\
\hline NMS & 3 & 430444 & 705801 & \multicolumn{3}{|c|}{ SEWALL FRANK } & -- & 60 & -- & -- & -- & -- & -- & Sp \\
\hline NMT & 1 & 430433 & 705717 & LOISELLE $\mathbf{R}$ & $\mathbf{M}$ & & -- & 60 & 40 & -- & $\mathbf{R}$ & -- & -- & Dug \\
\hline NMW & 2 & 430456 & 705823 & BERGERON HE & ARRY & & 1940 & 130 & 2 & -- & $\mathbf{s}$ & -- & -- & Tsh \\
\hline NMT & 3 & 430455 & 705818 & LANG CHARLE & ES $W$ & & -- & 110 & 36 & -- & $\mathbf{R}$ & -- & -- & Dug \\
\hline NMW & 4 & 430458 & 705709 & NEWMARKET, & TOWN & OF & 1914 & 50 & 6 & -- & $\mathbf{s}$ & -- & -- & BrW \\
\hline NMW & 6 & 430459 & 705835 & NEFMARKET, & TOWN & OF & 1974 & 100 & 24 & 38.0 & $\mathbf{s}$ & G & 48.0 & -- \\
\hline NMH & 9 & 430429 & 705905 & - & & & 1985 & 108 & 2 & 22.1 & $\mathbf{P}$ & $\mathbf{S}$ & 24.6 & Bor \\
\hline NMN & 10 & 430427 & 705642 & - & & & 1985 & 50 & 2 & 51.5 & $\mathbf{P}$ & $\mathbf{P}$ & 54.0 & Bor \\
\hline NMH & 11 & 430449 & 705807 & - & & & 1985 & 88 & 2 & 51.5 & $\mathbf{P}$ & $\mathbf{P}$ & 54.0 & Bor \\
\hline NMT & 12 & 430450 & 705810 & NEWMARKET, & TOFN & OF & 1983 & 90 & 3 & 69.5 & $\mathbf{S}$ & $\mathbf{P}$ & 75.5 & Wsh \\
\hline NMA & 13 & 430405 & 705840 & MALASKY & & & 1984 & 80 & -- & -- & -- & $\mathbf{x}$ & -- & BrW \\
\hline NMH & 14 & 430407 & 705843 & DARLING & & & 1984 & 80 & -- & -- & - & $\mathbf{x}$ & -- & Brit \\
\hline NMH & 15 & 430408 & 705844 & SCHULTZE & & & 1985 & 80 & - & -- & - & $\mathbf{x}$ & -- & Brf \\
\hline NMT & 16 & 430410 & 705848 & BRISTOL & & & 1985 & 90 & -- & -- & - & $\mathbf{x}$ & -- & Brî \\
\hline NMN & 17 & 430414 & 705852 & TARNOWSKI & & & 1984 & 100 & -- & -- & $-\infty$ & $\mathbf{x}$ & -- & Bry \\
\hline NMA & 18 & 430416 & 705853 & COFFEY & & & 1985 & 100 & -- & -- & -- & $\mathbf{x}$ & -- & BrN \\
\hline NMN & 19 & 430420 & 705855 & MALASKY & & & 1984 & 100 & - & -- & - & $\mathrm{x}$ & -- & Brw \\
\hline NMW & 20 & 430422 & 705857 & ANDERSON & & & 1984 & 90 & -- & -- & -- & $\mathbf{x}$ & -- & BrW \\
\hline NMH & 21 & 430448 & 705919 & MESERVEY & & & 1984 & 90 & -- & -- & -- & $\mathbf{x}$ & -- & Brw \\
\hline NMW & 22 & 430500 & 705346 & LEGACY & & & 1984 & 40 & - & -- & -- & $\mathbf{x}$ & -- & Brw \\
\hline NMN & 23 & 430459 & 705338 & MUSKOFF & & & 1985 & 40 & - & -- & -- & $\mathbf{x}$ & -- & Brw \\
\hline NMN & 24 & 430318 & 710012 & BOLAND & & & 1984 & 130 & -- & -- & -- & $\mathbf{x}$ & -- & Brif \\
\hline NMW & 25 & 430428 & 705816 & NEWMARKET, & TOWN & OF & 1964 & 80 & -- & -- & -- & -- & -- & -- \\
\hline NMW & 26 & 430446 & 705802 & NEWMARKET, & TOWN & OF & 1983 & 70 & -- & -- & -- & -- & -- & Irsh \\
\hline NMW & 27 & 430454 & 705800 & NEWMARKET, & TOWN & OF & 1964 & 120 & -- & -- & -- & -- & -- & -- \\
\hline NMA & 28 & 430423 & 705916 & NEWMARKET, & TOWN & $\mathrm{OF}$ & 1985 & 107.9 & - & - & -- & -- & -- & -- \\
\hline NMN & 29 & 430451 & 705857 & NEMMARKET, & TOWN & OF & 1964 & 110 & -- & -- & -- & -- & -- & -- \\
\hline NMW & 30 & 430420 & 705920 & NEWMARKET, & TOWN & OF & 1985 & 88.4 & -- & -- & -- & -- & -- & -- \\
\hline NMN & 31 & 430425 & 705919 & NEWMARKET, & TOWN & OF & 1985 & 100 & -- & -- & -- & $-\infty$ & $-\infty$ & Brw \\
\hline NMW & 32 & 430447 & 705846 & NEMMARKET, & TOWN & OF & 1964 & 150 & -- & -- & -- & -- & -- & -- \\
\hline NMN & 33 & 430451 & 705812 & NEWMARKET, & TOWN & OF & 1983 & 100 & 3 & 49.0 & $\mathbf{s}$ & $\mathbf{P}$ & 55.0 & Nish \\
\hline NMN & 34 & 430450 & 705810 & NEMMARKET, & TOWN & OF & 1983 & 100 & 3 & 65.0 & $\mathbf{s}$ & $\mathbf{P}$ & 71.0 & Wsh \\
\hline NMN & 35 & 430427 & 705918 & NEMMARKET, & TOWN & OF & 1985 & 91.1 & -- & -- & -- & -- & -- & Bri \\
\hline NMN & 36 & 430449 & 705658 & NEWMARKET, & TOWN & OF & 1965 & 40 & -- & -- & -- & -- & -- & -- \\
\hline NMW & 37 & 430444 & 705656 & NEMMARKET, & TOWN & OF & 1965 & 70 & -- & -- & -- & -- & -- & 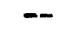 \\
\hline NMN & 38 & 430441 & 705654 & NEWMARKET, & TOWN & OF & 1965 & 70 & -- & -- & -- & -- & -- & -- \\
\hline NMN & 39 & 430424 & 70.5924 & NEKMARKET, & TOWN & OF & 1985 & 90 & -- & -- & -- & -- & -- & -- \\
\hline NMW & 40 & 430417 & 705859 & NEWMARKET, & TOWN & OF & 1965 & 100 & -- & -- & -- & -- & -- & -- \\
\hline NMW & 41 & 430427 & 705905 & NEMMARKET, & TOWN & OF & 1965 & 100 & -- & -- & -- & -- & -- & -- \\
\hline NMW & 42 & 430433 & 705722 & NEWMARKET, & TOWN & OF & 1965 & 60 & -- & -- & -- & -- & - & 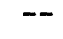 \\
\hline
\end{tabular}




\section{and springs--Continued}

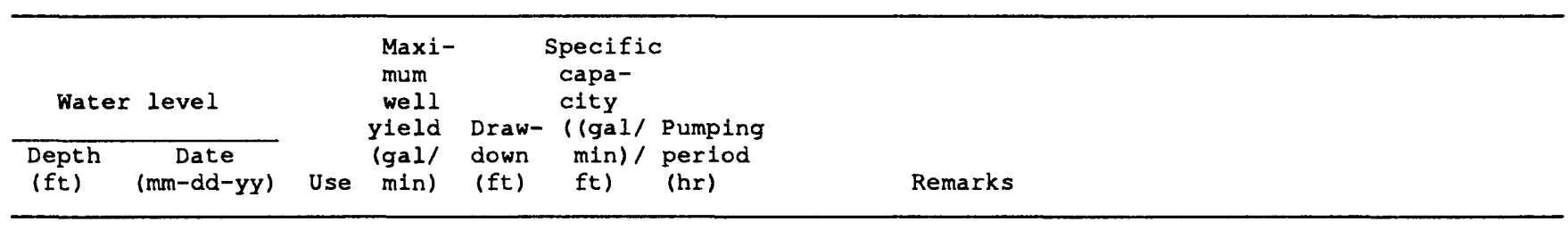

Newmarket--Continued

\begin{tabular}{|c|c|c|c|c|c|c|}
\hline -- & -- & $T$ & -- & - & -- & $\cdots$ \\
\hline -- & -- & $T$ & -- & - & -- & - \\
\hline & -- & $T$ & -- & $\cdots$ & -- & - \\
\hline 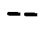 & -- & $T$ & -- & $\cdots$ & -- & - \\
\hline 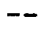 & -- & $T$ & -- & - & -- & - \\
\hline-- & -- & $\mathrm{T}$ & -- & - & -- & - \\
\hline & -- & H & 11 & - & -- & \\
\hline & -- & $\mathrm{H}, \mathrm{S}$ & 12 & - & -- & \\
\hline & -- & s & 55 & - & -- & 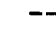 \\
\hline
\end{tabular}

$\begin{array}{lllllll}6.5 & 06-17-54 & \mathrm{H} & -- & - & - & -\end{array}$

$\begin{array}{lllllll}44 & 06-17-54 & \mathrm{H} & -- & - & -- & - \\ 16.3 & 01-01-40 & \text { H } & 20 & -- & -- & -- \\ -3 & 09-13-55 & \text { H } & -- & -- & -- & --\end{array}$

$\begin{array}{lllllll}16.3 & 09-13-55 & \text { H } & -- & -- & -- & -- \\ -3 & 09-13-55 & \text { P } & 84 & -- & -- & --\end{array}$

$\begin{array}{lllllll}35 & 06-07-77 & \text { P } & 200 & 24.2 & 8.3 & --\end{array}$

$\begin{array}{lllllll}0.8 & 01-27-86 & 0 & -- & -- & -- & --\end{array}$

$\begin{array}{lllllll}8.7 & 12-04-85 & 0 & -- & -- & -- & -\end{array}$

$\begin{array}{lllllll}9.3 & 01-29-86 & 0 & -- & -- & -- & -- \\ 8.8 & 11-10-83 & 0 & -- & -- & -- & -\end{array}$

USGS, CA

USGS, CA

$\begin{array}{lcrrrrc}4 & 04-09-84 & \text { H } & 60 & -- & -- & .1 \\ -- & -- & \text { H } & 8 & -- & -- & -- \\ -- & -- & \text { H } & 10 & -- & -- & -- \\ -- & -- & \text { H } & 10 & -- & -- & 1.5 \\ -- & -- & \text { H } & 8 & -- & -- & .1\end{array}$

$\begin{array}{ccccccc}-- & -- & \text { H } & 2.8 & -- & -- & -- \\ 12 & 04-09-84 & \text { H } & 8 & -- & -- & .2 \\ -- & -- & \text { H } & 7 & -- & -- & -- \\ -- & -- & \text { H } & 4 & -- & -- & -- \\ 15 & 02-02-84 & \text { H } & 0.8 & -- & -- & 8\end{array}$

$\begin{array}{ccccccc}-- & -- & \text { H } & 6 & -- & -- & -- \\ -- & -- & \text { H } & 8 & -- & -- & -- \\ 21.7 & 09-18-64 & 0 & -- & -- & -- & -- \\ 3.7 & 11-11-83 & 0 & -- & -- & -- & -- \\ -- & 09-25-64 & 0 & 5 & -- & -- & .1\end{array}$

WELL DESTROYED, CASING PULLED

WELL DESTROYED, CASING PULLED

WELL DESTROYED

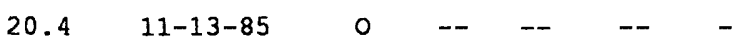

$09-29-64$

$2.2 \quad 11-13-85$

33 - $10-\overline{0}-64$

$\begin{array}{llll}15 & 1.5 & - & .1\end{array}$

WELL DESTROYED

$\begin{array}{cccc}15 & -- & -- & -- \\ -- & -- & -1 & -1\end{array}$

$19.802011-02-83$

$\begin{array}{llll}-- & -- & -- & - \\ -- & -- & -- & -\end{array}$

WELL DESTROYED

$\begin{array}{rr}9.8 & 11-02-83 \\ 12.2 & 11-10-83\end{array}$

$\begin{array}{rr}12.2 & 11-10-83 \\ 4.3 & 11-13-85\end{array}$

$10.2 \quad 03-16-65$

$13.2 \quad 03-17-65$

$11.7 \quad 03-17-65$

$11-13-85$

$03-18-65$

$03-19-65$

$8.7 \quad 03-23-65$

$\begin{array}{cccc}33 & -- & -- & 6 \\ 55 & -- & -- & 3 \\ -- & -- & -- & -- \\ 15 & -- & -- & -- \\ 7 & -- & -- & -- \\ -- & -- & -- & -- \\ -- & -- & -- & -- \\ 30 & -- & -- & -- \\ 2.5 & -- & -- & -- \\ -- & -- & -- & --\end{array}$

CASING PULLED

WELL DESTROYED, CASING PULLED

WELL DESTROYED, CASING PULLED

WELL DESTROYED

WELL DESTROYED, CASING PULLED

WELL DESTROYED, CASING PULLED 
Table 2.--Description of wells, borings,

\begin{tabular}{|c|c|c|c|c|c|c|c|c|c|c|c|}
\hline $\begin{array}{l}\text { Local } \\
\text { site } \\
\text { number }\end{array}$ & $\begin{array}{l}\text { Lati- } \\
\text { tude }\end{array}$ & $\begin{array}{l}\text { Long- } \\
\text { tude }\end{array}$ & Owner or user & $\begin{array}{l}\text { Year } \\
\text { comp- } \\
\text { leted }\end{array}$ & $\begin{array}{l}\text { Altitude } \\
\text { above } \\
\text { sea } \\
\text { level } \\
\text { (ft) }\end{array}$ & $\begin{array}{l}\text { Diam- } \\
\text { eter } \\
\text { of } \\
\text { well } \\
\text { (in.) }\end{array}$ & $\begin{array}{c}\text { Depth to } \\
\text { bottom } \\
\text { of } \\
\text { casing } \\
\text { (ft) }\end{array}$ & $\begin{array}{l}\text { Casing } \\
\text { mate- } \\
\text { rial }\end{array}$ & $\begin{array}{l}\text { Type } \\
\text { of } \\
\text { fin- } \\
\text { ish }\end{array}$ & $\begin{array}{l}\text { Jepth to } \\
\text { bottom } \\
\text { of } \\
\text { open } \\
\text { section } \\
\text { (ft) }\end{array}$ & $\begin{array}{l}\text { Type } \\
\text { of } \\
\text { site }\end{array}$ \\
\hline
\end{tabular}

Newmarket--Cont inued

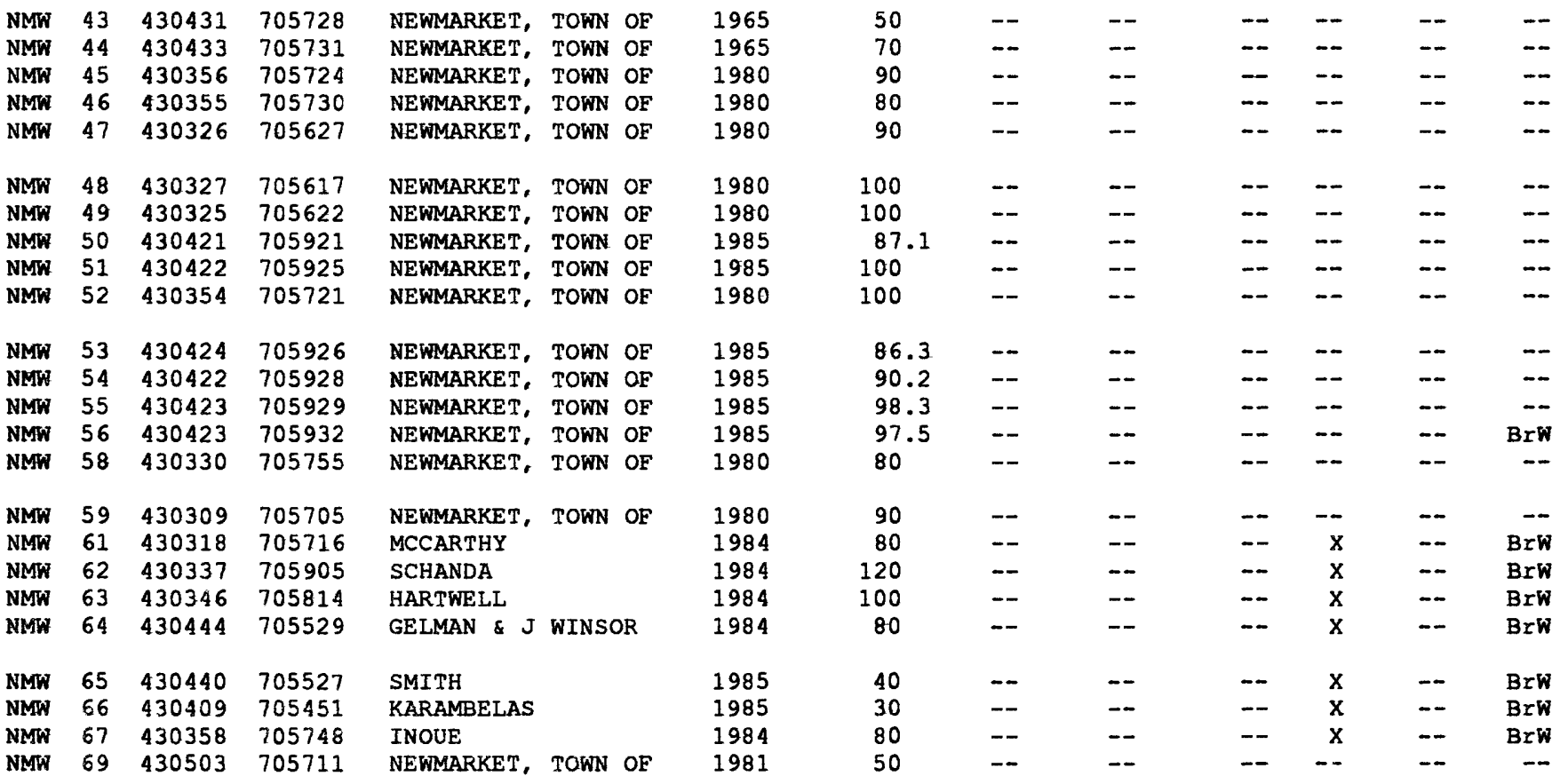

NWW $\quad 2 \quad 431156 \quad 710750$

NWW $4 \quad 431136 \quad 710854$

NWW $\quad 5 \quad 431136 \quad 710854$
BATEMAN C E

HARDY H A

HARDY H A

Northwood

$\begin{array}{cccccccc}1960 & 470 & -- & -- & -- & 0 & -- & \text { Dug } \\ 1965 & 565 & 6 & 25.0 & \text { s } & \text { X } & 110.0 & \text { BrW } \\ -- & 565 & -- & -- & -- & -- & -- & --\end{array}$

Nottingham

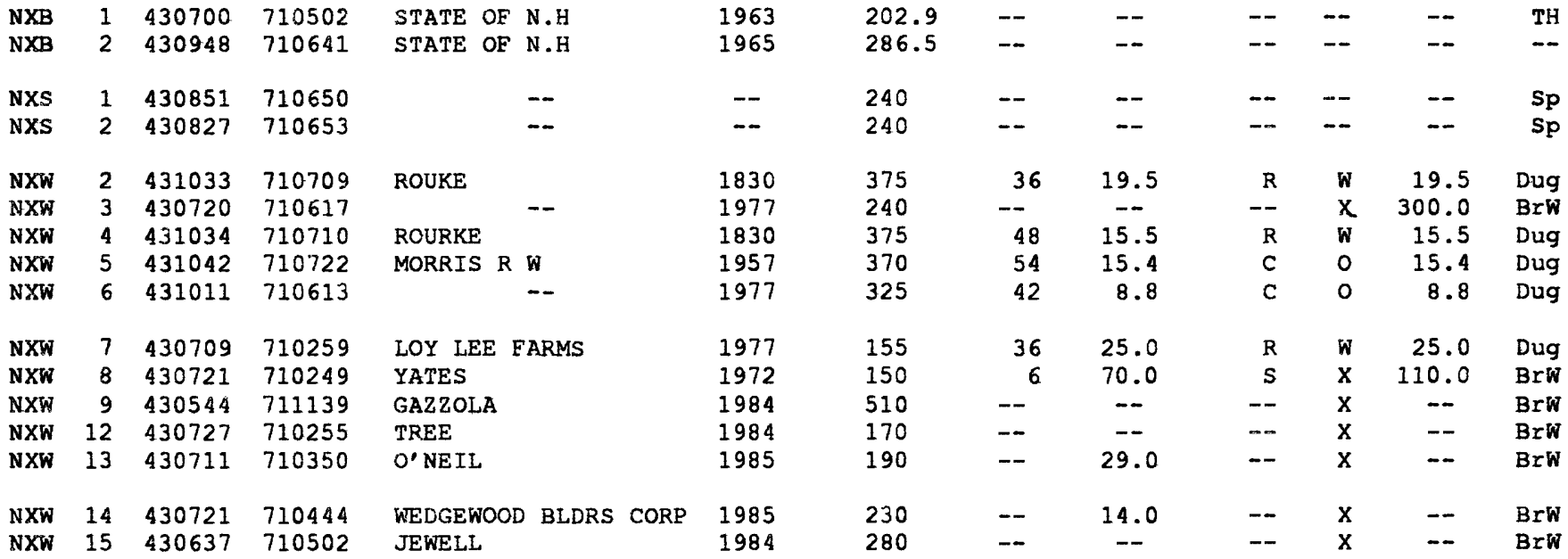




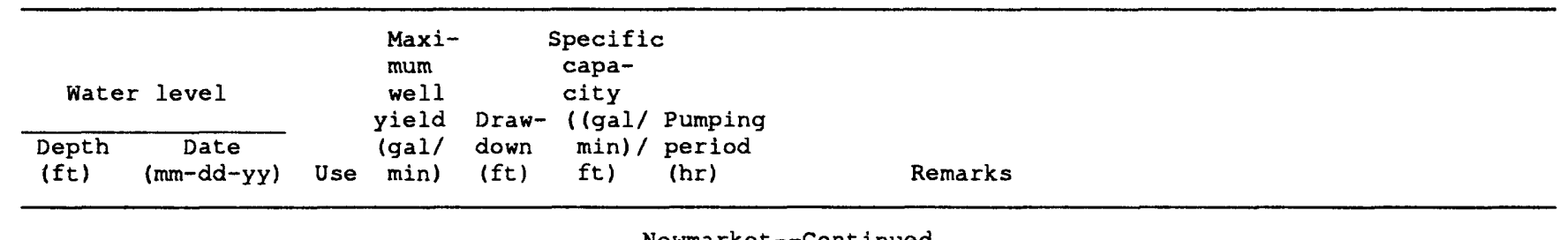

Newmarket--Continued

$\begin{array}{cccccccc}8.3 & 03-23-65 & 0 & 25 & -- & -- & -- & \text { WELL DESTROYED, CASING PULLED } \\ 3.8 & 03-24-65 & 0 & 5 & -- & -- & -- & \text { WELL DESTROYED, CASING PULLED } \\ 9 & 09-27-80 & 0 & 45 & -- & -- & -- & \text { WELL DESTROYED, CASING PULLED } \\ 1.5 & 09-30-80 & 0 & -- & -- & -- & -- & \text { WELL DESTROYED, CASING PULLED } \\ 24 & 10-01-80 & 0 & -- & -- & -- & -- & \text { WELL DESTROYED, CASING PULLED } \\ 26.5 & 10-02-80 & 0 & - & -- & -- & -- & \text { WELL DESTROYED, CASING PULLED } \\ 23.2 & 10-02-80 & 0 & -- & -- & -- & -- & \text { WELL DESTROYED, CASING PULLED } \\ 1.9 & 11-13-85 & 0 & -- & -- & -- & -- & \\ -- & 10-07-80 & 0 & -- & -- & -- & -- & \text { WELL DESTROYED, CASING PULLED } \\ 23.3 & 10-07-80 & -- & -- & - & - & \\ 2.9 & 11-13-85 & 0 & -- & -- & -- & -- & \\ 3.7 & 11-13-85 & 0 & -- & -- & -- & -- & \\ 12.4 & 11-13-85 & 0 & -- & -- & -- & -- & \\ 9.8 & 11-13-85 & 0 & -- & -- & -- & -- & 23 \text { INCHES OF VACUUM } \\ 1.5 & 12-24-80 & 0 & -- & -- & -- & -- & \\ 1.6 & 12-29-80 & 0 & -- & -- & -- & -- & \text { WELL DESTROYED, CASING PULLED } \\ -- & -- & \mathrm{H} & 30 & -- & -- & -- & \end{array}$

Northwood

$\begin{array}{cccccccc}9 & 10-16-69 & \mathrm{H} & -- & -- & -- & -- & \\ 15 & 00-00-66 & \mathrm{H} & 12 & 15 & -- & -- & \text { FILTERED FOR IRON } \\ 8.3 & 10-22-69 & 0 & -- & -- & -- & -- & \end{array}$

Nottingham

$\begin{array}{lccccccc}-- & - & \text { T } & -- & -- & -- & -- \\ -- & -- & \text { T } & -- & -- & -- & -- \\ -- & -- & \text { H } & -- & -- & -- & -- \\ -- & - & \text { H } & -- & -- & -- & -- & \\ 5.9 & 12-30-77 & \text { O } & -- & -- & -- & -- & \\ -- & -- & \text { H } & -- & -- & -- & -- & \text { RECENTLY PUMPED WELL } \\ 9.7 & 12-30-77 & \text { H } & -- & -- & -- & -- & \\ -- & -- & \text { H } & -- & -- & -- & -- & \\ 7 & 12-00-77 & \text { H } & -- & -- & -- & -- & \\ 15 & 08-00-77 & \text { H } & -- & -- & -- & -- \\ -- & -- & \text { H } & 52 & -- & -- & -- \\ -- & -- & \text { H } & 1.5 & -- & -- & .2 \\ 20 & 03-08-84 & \text { H } & 10 & -- & -- & .2 \\ 1.5 & 05-30-85 & \text { H } & 2 & -- & -- & 3 \\ -- & -- & \text { H } & 6 & -- & -- & 1.5 \\ -- & -- & \text { H } & 4 & -- & -- & .2\end{array}$




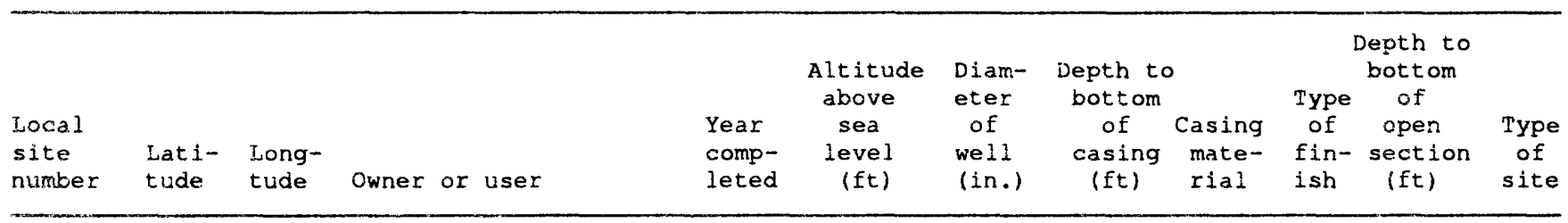

Nottingham--Continued

\begin{tabular}{|c|c|c|c|c|c|c|c|c|c|c|c|c|}
\hline NXW & 16 & 430541 & 710606 & COTE & 1984 & 370 & -- & -- & -- & $x$ & -- & Brw \\
\hline NXW & 17 & 430538 & 710604 & EOURNIER & 1984 & 370 & -- & -- & -- & $\mathrm{x}$ & -- & $\mathrm{BrW}$ \\
\hline NXW & 18 & 430551 & 710613 & PEAHLERT & 1985 & 470 & -- & 15.0 & $\cdots-$ & $x$ & -- & BrW \\
\hline NXW & 19 & 430610 & 710644 & EERNALD & 1985 & 460 & $-\infty$ & 100.0 & -- & $x$ & -- & BrW \\
\hline $\mathrm{HXW}$ & 20 & 430616 & 710635 & WELCH & 1985 & 470 & -- & 59.0 & - & $x$ & -- & BrW \\
\hline NXW & 21 & 430549 & 710604 & COMM UNIVERALIST $\mathrm{CH}$ & 1984 & 270 & $-\infty$ & -- & -- & $x$ & -- & BrW \\
\hline NXW & 22 & 430622 & 710654 & FERNALD & 1985 & 450 & -- & 30.0 & -- & $x$ & -- & BrW \\
\hline NXW & 23 & 430650 & 710640 & GAUTHIER & 1985 & 320 & -- & 30.0 & $-\infty$ & $\mathrm{x}$ & -- & BrW \\
\hline NXW & 24 & 430652 & 710639 & SCHOF IELD & 1984 & 310 & $-\infty$ & -- & -- & $x$ & -- & BrW \\
\hline NXW & 25 & 430658 & 710638 & KELLER & 1985 & 270 & -- & 33.0 & -- & $\mathrm{x}$ & -- & BrW \\
\hline NXW & 26 & 430653 & 710645 & KLEMARCZYK & 1985 & 310 & -- & 23.0 & - & $x$ & -- & BrW \\
\hline NXW & 27 & 430651 & 710644 & VIEIRA & 1985 & 320 & -- & 20.0 & -- & $\mathrm{x}$ & -- & BrW \\
\hline NXW & 28 & 430844 & 710249 & BALBEN & 1985 & 330 & -- & 29.0 & -- & $x$ & -- & $\mathrm{BrW}$ \\
\hline $\mathrm{NXW}$ & 29 & 430848 & 710254 & LOPEZ & 1985 & 330 & -- & 19.0 & - & $\mathrm{x}$ & -- & BrW \\
\hline NXW & 30 & 430859 & 710310 & OSGOOD & 1984 & 280 & -- & -- & -- & $x$ & -- & BrW \\
\hline NXW & 31 & 430820 & 710348 & WEINER & 1985 & 190 & -- & 31.0 & -- & $x$ & -- & Brw \\
\hline NXN & 32 & 430823 & 710347 & JEWELL & 1984 & 190 & -- & -- & -- & $x$ & -- & BrW \\
\hline NXW & 33 & 430839 & 710344 & ST AMOUR & 1985 & 190 & -- & 19.0 & -- & $\mathrm{x}$ & -- & Brw \\
\hline NXW & 34 & 430739 & 710523 & NELSON & 1985 & 350 & -- & 39.0 & -- & $\mathrm{x}$ & -- & Brw \\
\hline NXW & 35 & 430859 & 710403 & CALIIORAS & 1984 & 180 & -- & -- & -- & $\mathrm{x}$ & -- & Brw \\
\hline$N X W$ & 36 & 430920 & 710354 & GROUT & 1985 & 230 & -- & 39.0 & -- & $x$ & -- & BrW \\
\hline NXW & 37 & 430916 & 710352 & NEWTON & 1985 & 200 & -- & 30.0 & -- & $\mathrm{x}$ & -- & BrW \\
\hline $\mathrm{NXW}$ & 38 & 430918 & 710347 & HANSON & 1984 & 230 & $\cdots$ & -- & -- & $x$ & -- & BrW \\
\hline NXW & 40 & 430909 & 710656 & GULEZIAN & 1984 & 240 & -- & -- & -- & $x$ & -- & BrW \\
\hline NXW & * 1 & 430959 & 710623 & LIBERTY BAPTIST CHRCH & 1985 & 360 & -- & -- & -- & $x$ & -- & $B r W$ \\
\hline NXW & 42 & 430935 & 710737 & EVARTS & 1985 & 270 & -- & 20.0 & -- & $x$ & -- & BrW \\
\hline NXW & 43 & 430807 & 710830 & BROOKS & 1985 & 370 & -- & -- & $-\infty$ & $x$ & -- & $\mathrm{Brw}$ \\
\hline$N X \bar{W}$ & 44 & 430808 & 710827 & GEHRKE & 1985 & 380 & -- & 19.0 & -- & $\mathrm{x}$ & -- & $B r W$ \\
\hline $\mathrm{NXW}$ & 45 & 430806 & 710829 & WOLFE & 1985 & 380 & -- & 30.0 & -- & $x$ & -- & BIW \\
\hline NXW & 46 & 430806 & 710826 & NORTON & 1984 & 380 & -- & -- & -- & $x$ & -- & $\mathrm{BrW}$ \\
\hline NXW & 47 & 430757 & 710821 & PEARSON & 1985 & 330 & -- & 41.0 & -- & $\mathrm{x}$ & -- & $\mathrm{BrW}$ \\
\hline NXW & $\$ 8$ & 431000 & 710505 & DEANE & 1985 & 410 & -- & 9.0 & -- & $\mathrm{x}$ & -- & $B r W$ \\
\hline NXW & 50 & 430906 & 710609 & COLE & 1984 & 250 & -- & -- & -- & $\mathrm{x}$ & -- & BrW \\
\hline NXW & 51 & 430910 & 710601 & COLE & 1984 & 290 & -- & -- & -- & $x$ & -- & BrW \\
\hline NXW & 52 & 430702 & 710530 & CLEMENTS & 1985 & 210 & -- & 19.0 & -- & $x$ & -- & $\mathrm{BrW}$ \\
\hline NXW & 53 & 430411 & 710834 & BUTLER & 1984 & 190 & -- & -- & -- & $x$ & $-\infty$ & $\mathrm{BrW}$ \\
\hline NXW & 54 & 431156 & 710742 & LEMAY & 1984 & 460 & -- & -- & -- & $\mathrm{x}$ & -- & BrW \\
\hline NXW & 55 & 430510 & 710947 & WATTS & 1984 & 255 & -- & -- & -- & $\mathrm{x}$ & -- & BrW \\
\hline NXW & 56 & 430409 & 710914 & HORIZON HOMES & 1984 & 280 & -- & -- & -- & $x$ & -- & $\mathrm{BrW}$ \\
\hline NXW & 57 & 430359 & 710838 & DONIGAN CONST & 1984 & 200 & -- & -- & -- & $\mathrm{x}$ & -- & $\mathrm{BrW}$ \\
\hline NXW & 58 & 430554 & 710743 & WHARTON & 1984 & 290 & -- & -- & -- & $\mathrm{x}$ & -- & $\mathrm{BrW}$ \\
\hline NXW & 59 & 430948 & 710758 & WEST & 1985 & 330 & -- & 48.0 & -- & $\mathrm{x}$ & -- & $\mathrm{BrW}$ \\
\hline NXW & 60 & 430505 & 710745 & WHITE & 1985 & 330 & -- & 4.0 & -- & $x$ & -- & BrW \\
\hline NXW & 61 & 430950 & 710801 & NAWROCKI & 1985 & 330 & -- & 59.0 & -- & $x$ & -- & $\mathrm{BrW}$ \\
\hline NXW & 62 & 430355 & 710842 & HATT & 1985 & 200 & -- & 19.0 & -- & $\mathrm{x}$ & -- & BrW \\
\hline NXW & 63 & 430438 & 710759 & REED & 1985 & 230 & -- & 80.0 & -- & $x$ & -- & BrW \\
\hline NXW & 64 & 431105 & 710826 & ELLIS & 1985 & 420 & -- & 65.0 & -- & $\mathbf{x}$ & -- & BrW \\
\hline NXW & 65 & 430504 & 710956 & BECK & 1985 & 310 & -- & 19.0 & -- & $\mathrm{x}$ & -- & $\mathrm{Brw}$ \\
\hline NXW & 66 & 430522 & 710803 & BOUDROT & 1985 & 260 & -- & 19.0 & -- & $x$ & -- & BrW \\
\hline NXW & 67 & 430523 & 710807 & MOREL & 1985 & 250 & -- & 29.0 & $-\infty$ & $x$ & -- & $B r W$ \\
\hline
\end{tabular}


and springs--Continued

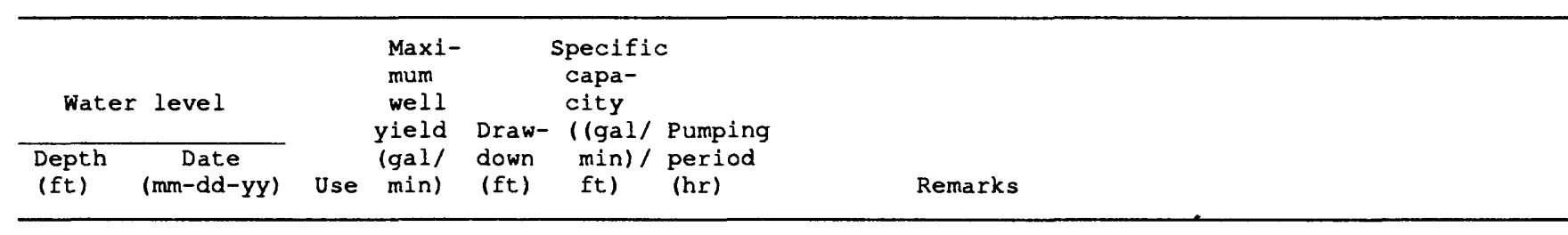

\section{Nott ingham--Cont inued}

\begin{tabular}{|c|c|c|c|c|c|c|c|}
\hline-- & -- & $\mathrm{H}$ & 3.5 & -- & -- & .2 & \\
\hline-- & -- & $\mathrm{H}$ & 5 & -- & -- & -- & \\
\hline$-\infty$ & -- & $\mathrm{H}$ & 1 & -- & -- & -- & \\
\hline-- & -- & $z$ & 10 & -- & -- & 3 & \\
\hline 12 & $08-21-85$ & $\mathrm{H}$ & 4 & -- & -- & .2 & \\
\hline 15 & $06-11-84$ & $T$ & 5 & -- & -- & 1 & \\
\hline-- & -- & $\mathrm{H}$ & 3 & -- & -- & 2 & \\
\hline-- & -- & $\mathrm{H}$ & 4.5 & -- & -- & 1 & \\
\hline-- & -- & $\mathrm{H}$ & 3 & -- & -- & 2 & \\
\hline 14 & $06-05-85$ & $\mathrm{H}$ & 3 & -- & -- & 2 & \\
\hline 25 & $06-07-85$ & $\mathrm{H}$ & 20 & -- & -- & 1 & \\
\hline-- & -- & $\mathrm{H}$ & 0.3 & -- & -- & 1.5 & \\
\hline-- & -- & $\mathrm{H}$ & 15 & -- & -- & 1 & \\
\hline 12 & $05-09-85$ & $\mathrm{H}$ & 2 & -- & -- & .2 & \\
\hline-- & -- & $\mathrm{H}$ & 4 & -- & -- & -- & \\
\hline-- & -- & $\mathrm{H}$ & 12 & -- & -- & 2 & \\
\hline-- & -- & $\mathrm{H}$ & 10 & -- & -- & 1 & \\
\hline-- & -- & $\mathrm{H}$ & 45 & -- & -- & 1 & \\
\hline 20 & $06-07-85$ & $\mathrm{H}$ & 4 & -- & -- & 2 & \\
\hline-- & -- & $\mathrm{H}$ & -- & -- & -- & -- & \\
\hline 25 & $05-22-85$ & $\mathrm{H}$ & 5 & -- & -- & 1 & \\
\hline 20 & $05-22-85$ & $\mathrm{H}$ & 30 & -- & -- & 1 & \\
\hline-- & -- & $\mathrm{H}$ & 6 & -- & -- & 1 & \\
\hline 13 & $04-13-84$ & $\mathrm{H}$ & 8 & -- & -- & -- & \\
\hline-- & -- & $\mathrm{T}$ & 4 & -- & -- & .2 & \\
\hline-- & -- & $\mathrm{H}$ & 1 & -- & -- & 1.5 & \\
\hline-- & -- & $\mathrm{H}$ & 30 & -- & -- & .5 & \\
\hline-- & -- & $\mathrm{H}$ & 1 & -- & -- & .7 & \\
\hline-- & -- & $\mathrm{H}$ & 60 & -- & -- & 1 & \\
\hline 12 & $12-17-84$ & $\mathrm{H}$ & 1.5 & -- & -- & .2 & \\
\hline 25 & $09-10-85$ & $\mathrm{H}$ & 25 & -- & -- & .5 & \\
\hline-- & -- & $\mathrm{H}$ & 6 & -- & -- & 1.7 & \\
\hline-- & -- & $\mathrm{H}$ & 30 & -- & -- & -- & \\
\hline 8 & $06-11-84$ & $\mathbf{H}$ & 4 & -- & -- & -- & \\
\hline 20 & $08-22-85$ & H & 8 & -- & -- & .2 & \\
\hline 6 & $02-23-84$ & $\mathrm{H}$ & 3 & -- & -- & -- & \\
\hline 10 & $04-12-84$ & H & 20 & -- & -- & .2 & \\
\hline 6 & $07-31-84$ & H & 10 & -- & -- & -- & \\
\hline-- & -- & $\mathrm{H}$ & 2.5 & -- & -- & .5 & \\
\hline-- & -- & $\mathrm{H}$ & 4 & - & -- & .2 & \\
\hline-- & -- & H & 0.3 & -- & -- & -- & \\
\hline-- & -- & $\mathrm{H}$ & 12 & - & -- & 1 & \\
\hline-- & -- & $\mathrm{H}$ & 60 & -- & -- & 1 & FLOWING WELI \\
\hline-- & -- & $\mathrm{H}$ & 20 & -- & -- & -- & \\
\hline-- & -- & H & 5 & - & -- & 1 & \\
\hline 48 & $06-01-85$ & $H$ & 25 & -- & -- & 1 & \\
\hline 18 & $09-14-85$ & $\mathbf{H}$ & 5.5 & -- & -- & 1 & \\
\hline-- & -- & $\mathrm{H}$ & 30 & -- & -- & .2 & \\
\hline-- & -- & $\mathrm{H}$ & 4 & -- & -- & 1 & \\
\hline-- & -- & $\mathrm{H}$ & 7.5 & -- & -- & 1 & \\
\hline
\end{tabular}




\begin{tabular}{|c|c|c|c|c|c|c|c|c|c|c|c|c|}
\hline \multicolumn{2}{|c|}{$\begin{array}{l}\text { Local } \\
\text { site } \\
\text { number }\end{array}$} & \multirow[t]{2}{*}{$\begin{array}{l}\text { Lati- } \\
\text { tude }\end{array}$} & \multirow[t]{2}{*}{$\begin{array}{l}\text { Long- } \\
\text { tude }\end{array}$} & ner or user & \multirow{2}{*}{$\begin{array}{l}\begin{array}{l}\text { Year } \\
\text { comp- } \\
\text { leted }\end{array} \\
\text { Raym }\end{array}$} & \multirow[t]{2}{*}{$\begin{array}{l}\text { Altitude } \\
\text { above } \\
\text { sea } \\
\text { level } \\
\text { (ft) }\end{array}$} & \multirow[t]{2}{*}{$\begin{array}{l}\text { Diam- } \\
\text { eter } \\
\text { of } \\
\text { well } \\
\text { (in.) }\end{array}$} & \multirow[t]{2}{*}{$\begin{array}{l}\text { Depth to } \\
\text { bott om } \\
\text { of } \\
\text { casing } \\
\text { (ft) }\end{array}$} & \multirow[t]{2}{*}{$\begin{array}{l}\text { Casing } \\
\text { mate- } \\
\text { rial }\end{array}$} & \multirow{2}{*}{\multicolumn{2}{|c|}{$\begin{array}{l}\text { Depth to } \\
\text { bottom } \\
\text { of } \\
\text { open } \\
\text { - section } \\
\text { (ft) }\end{array}$}} & \multirow[t]{2}{*}{$\begin{array}{l}\text { Type } \\
\text { of } \\
\text { site }\end{array}$} \\
\hline & & & & & & & & & & & & \\
\hline RBB & 1 & 430346 & 711342 & NHDOT & 1973 & 206.2 & -- & -- & -- & -- & -- & Wsh \\
\hline RBB & 2 & 430141 & 711007 & NHDOT & 1971 & 180.0 & -- & -- & -- & -- & -- & Wsh \\
\hline RBB & 3 & 430144 & 710958 & NHDOT & 1971 & 178.2 & -- & -- & -- & -- & -- & Wsh \\
\hline RBB & 4 & 430223 & 711030 & NHDOT & 1947 & 170 & -- & -- & -- & -- & -- & Wsh \\
\hline RBW & 4 & 430132 & 710918 & PINE ACRES CAMPGROUND & 1960 & 180 & 42 & 8.1 & C & 0 & -- & Dug \\
\hline RBW & 5 & 430134 & 710912 & PINE ACRES CAMPGROUND & 1960 & 170 & 42 & 8.2 & c & 0 & -- & Dug \\
\hline RBW & 6 & 430327 & 711314 & LEISURE VILIAGE & 1977 & 230 & 60 & 15.0 & c & 0 & -- & Dug \\
\hline RBW & 7 & 430436 & 711326 & GARLAND & 1940 & 220 & 24 & 11.9 & $\mathbf{R}$ & $\mathrm{x}$ & 11.9 & Dug \\
\hline RBW & 8 & 430024 & 711012 & -- & 1984 & 176 & 2 & 35.2 & $\mathrm{P}$ & $\mathbf{P}$ & 37.2 & Bor \\
\hline RBW & 9 & 430025 & 711004 & -- & 1984 & 172 & 2 & 9.0 & $\mathrm{P}$ & $\mathrm{P}$ & 11.0 & Bor \\
\hline RBW & 10 & 430028 & 710949 & -- & 1984 & 175 & 2 & 12.0 & P & P & 14.0 & Bor \\
\hline RBW & 11 & 430027 & 710944 & -- & 1984 & 175 & 2 & 19.0 & $P$ & $P$ & 21.0 & Bor \\
\hline RBW & 13 & 430027 & 710949 & KEITH & 1972 & 175 & 48 & 11.2 & C & -- & -- & Dug \\
\hline RBW & 14 & 430027 & 710944 & ROSS JIM & 1984 & 175 & -- & -- & -- & $\mathrm{x}$ & -- & Dug \\
\hline RBW & 16 & 430028 & 710939 & AMES RALPH & 1969 & 175 & -- & -- & -- & 0 & -- & Dug \\
\hline RBW & 26 & 430137 & 711006 & -- & 1984 & 200 & 6 & 20.0 & $\mathbf{s}$ & $\mathrm{x}$ & -- & BrW \\
\hline RBW & 27 & 430136 & 711009 & ROWLINSON & 1964 & 220 & -- & -- & -- & $x$ & -- & -- \\
\hline RBW & 28 & 430134 & 711007 & MAYNARD & 1973 & 195 & 6 & 20.0 & $\mathbf{s}$ & $\mathrm{x}$ & -- & $\mathrm{BrW}$ \\
\hline RBW & 30 & 430126 & 711007 & LOUIS M & 1980 & 180 & -- & -- & -- & $\mathrm{x}$ & -- & $\mathrm{BrW}$ \\
\hline RBW & 31 & 430247 & 711230 & BECK & 1975 & 195 & 48 & 12.0 & c & 0 & -- & Dug \\
\hline RBW & 33 & 430241 & 711220 & LACZINSKI ALBERT & 1954 & 205 & -- & -- & -- & 0 & -- & Dug \\
\hline RBW & 34 & 430414 & 711333 & WOODHEAD $\mathbf{R}$ & 1964 & 210 & 6 & 8.0 & $\mathbf{s}$ & $T$ & -- & Dvn \\
\hline RBW & 35 & 430308 & 711251 & WELDY & 1978 & 220 & -- & -- & -- & 0 & -- & Dug \\
\hline RBW & 36 & 430308 & 711251 & WELDY & 1978 & 220 & -- & -- & -- & 0 & -- & $\mathrm{BrW}$ \\
\hline RBW & 37 & 430306 & 711249 & SAMARA $B$ & 1972 & 220 & 36 & 20.0 & $\mathbf{T}$ & 0 & -- & Dug \\
\hline RBW & 39 & 430306 & 711254 & CANTIN CARL & 1984 & 200 & -- & -- & -- & $\mathbf{T}$ & -- & Dvn \\
\hline RBW & 41 & 430351 & 711342 & TREANTAFEL G & 1946 & 225 & 48 & 12.0 & $T$ & 0 & $-\infty$ & Dug \\
\hline RBW & 42 & 430344 & 711356 & GODDARD A W & 1967 & 210 & -- & -- & -- & 0 & -- & Dug \\
\hline RBW & 46 & 425933 & 711138 & HOMER & 1958 & 185 & - & -- & -- & $\mathbf{x}$ & -- & $\mathrm{Cbl}$ \\
\hline RBW & 47 & 425930 & 711141 & MALONEY P & 1967 & 190 & -- & -- & -- & $x$ & -- & BrW \\
\hline RBW & 48 & 430201 & 711200 & -- & 1984 & 192 & 2 & 42.0 & $\mathrm{P}$ & $\mathbf{P}$ & 31.9 & Bor \\
\hline RBW & 49 & 430202 & 710927 & -- & 1984 & 188 & 2 & 14.0 & $\mathbf{P}$ & P & 16.0 & Bor \\
\hline RBW & 50 & 430200 & 711010 & -- & 1984 & 192 & 2 & 15.1 & $\mathrm{P}$ & $\mathbf{P}$ & 17.2 & Bor \\
\hline RBW & 51 & 430101 & 710919 & -- & 1984 & 180 & 2 & 18.7 & $\mathbf{P}$ & P & 20.7 & Bor \\
\hline RBW & 52 & 430110 & 710930 & -- & 1984 & 175 & 2 & 29.6 & $\mathbf{P}$ & $\mathbf{P}$ & 31.6 & Bor \\
\hline RBW & 53 & 430234 & 711241 & พOOD & 1985 & 240 & -- & -- & -- & $\mathbf{x}$ & -- & BrW \\
\hline RBW & 54 & 430233 & 711239 & NYE & 1985 & 230 & -- & -- & -- & $\mathrm{x}$ & -- & BrW \\
\hline
\end{tabular}




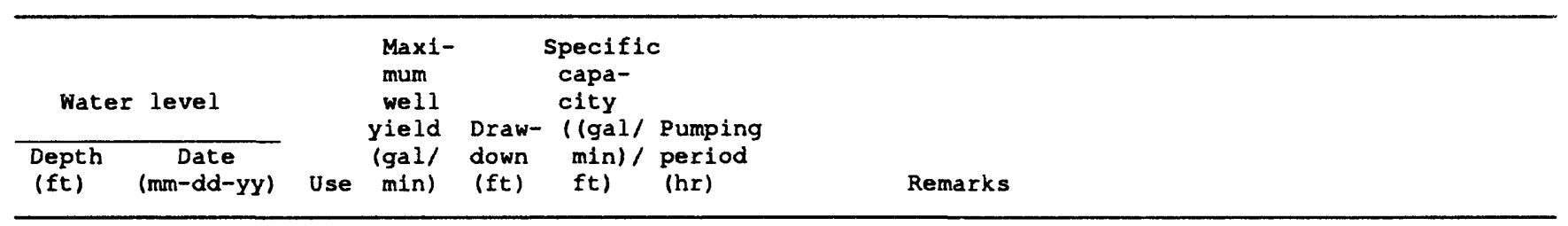

Rajmond

\begin{tabular}{|c|c|c|c|c|c|c|c|}
\hline-- & -- & $\mathbf{T}$ & -- & -- & -- & -- & USGS \\
\hline- & -- & $\mathrm{T}$ & -- & -- & -- & -- & \\
\hline-- & -- & $\mathrm{T}$ & -- & -- & -- & -- & \\
\hline- & -- & $\mathbf{T}$ & -- & -- & -- & -- & \\
\hline-- & -- & $\mathbf{T}$ & -- & -- & -- & -- & \\
\hline-- & -- & $\mathbf{T}$ & -- & -- & -- & -- & \\
\hline 20 & $00-00-35$ & $\mathbf{P}$ & -- & -- & -- & -- & PUMP ING WELL \\
\hline 13 & $01-01-80$ & $\mathbf{P}$ & 820 & 32.6 & 25.1 & 48 & \\
\hline 3 & $06-30-77$ & $\mathbf{P}$ & - & - & $\ldots$ & -- & PUMPING WELL \\
\hline 0.4 & $06-30-77$ & $\mathbf{P}$ & -- & -- & -- & -- & RECENTLY PUMPED WELL \\
\hline 1.8 & $06-30-77$ & $\mathrm{P}$ & -- & - & -- & -- & RECENTLY PUMPED WELL \\
\hline 10 & $07-00-77$ & $\mathbf{P}$ & -- & -- & -. & -- & \\
\hline 10.3 & $01-00-78$ & $\mathrm{H}$ & -- & $\ldots$ & -- & -- & \\
\hline 7.7 & $05-17-84$ & 0 & -- & - & -- & -- & USGS, CA \\
\hline 5.7 & $05-17-84$ & 0 & -- & -- & -- & -- & USGS, CA \\
\hline 3.5 & $05-17-84$ & 0 & -- & -- & -- & -- & USGS, CA \\
\hline 7.8 & $11-09-84$ & 0 & -- & -- & - & $-\infty$ & USGS, CA \\
\hline 3.6 & $05-17-84$ & $\mathrm{H}$ & -- & -- & -- & -- & \\
\hline 4.4 & $05-17-84$ & $\mathrm{H}$ & -- & $\ldots$ & -- & $-\infty$ & WATER FILTERED FOR IRON \\
\hline 2.9 & $05-17-84$ & $\mathrm{H}$ & -- & -- & -- & -- & \\
\hline-- & -- & $\mathrm{H}$ & 30 & -- & -- & -- & \\
\hline 5.7 & $05-17-84$ & $\mathrm{U}$ & -- & -- & -- & -- & \\
\hline 9.9 & $05-17-84$ & $\mathrm{H}$ & -- & -. & -- & -- & \\
\hline 1 & $05-18-84$ & $\mathrm{H}$ & -- & $-\infty$ & -- & -- & \\
\hline 19 & $05-18-84$ & $\mathrm{H}$ & -- & -- & -- & -- & \\
\hline 35 & $01-01-81$ & $\mathrm{H}$ & 40 & -- & -- & -- & \\
\hline 11.7 & $05-18-84$ & $\mathrm{H}$ & 35 & -- & -- & -- & \\
\hline 40 & $01-01-84$ & $\mathrm{H}$ & 15 & -- & -- & -- & \\
\hline -- & -- & $\mathrm{H}$ & - & - & - & -- & \\
\hline 14 & $01-01-73$ & $\mathrm{H}$ & 1.5 & -- & -- & -- & \\
\hline-- & -- & $\mathrm{H}$ & -- & -- & -- & -- & WATER TESTED WSPCC, FILTERED FOR IRON AND \\
\hline-- & -- & $\mathrm{H}$ & 35 & -- & -- & -- & WATER TESTED WSPCC, IRON AND MANGANESE \\
\hline-- & -- & $\mathrm{H}$ & -- & -- & -- & -- & \\
\hline 55 & $01-01-64$ & $\mathrm{H}$ & 15 & -- & -- & -- & \\
\hline 6 & $01-01-78$ & $\mathrm{U}$ & -- & -- & -- & --1 & \\
\hline-- & -- & $\mathrm{H}$ & -- & -- & -- & -- & \\
\hline 11.7 & $06-06-84$ & $\mathrm{H}$ & -- & -- & -- & -- & OWNER REPORTS IRON STAIN AND POOR TASTE \\
\hline-- & -- & $\mathrm{H}$ & -- & -- & -- & -- & WATER TESTED BY WSPCC \\
\hline 7 & $01-01-46$ & c & -- & -- & -- & -- & WATER TESTED WSPCC, HIGH IRON; HIGH YIELD \\
\hline-- & -- & $\mathrm{H}$ & -- & -- & -- & -- & WATER TESTED BY WSPCC \\
\hline-- & -- & $\mathrm{H}$ & -- & -- & -- & -- & WATER TESTED WSPCC \\
\hline-- & -- & $\mathrm{H}$ & -- & -- & -- & -- & WATER TESTED BY UNH \\
\hline 9.3 & $11-07-84$ & 0 & -- & -- & -- & -- & USGS, CA, ORGANIC LAYER ABOUT 29 FEET \\
\hline 6.1 & $11-08-84$ & 0 & -- & -- & -- & -- & USGS, CA \\
\hline 10.1 & $11-08-84$ & 0 & -- & -- & -- & -- & USGS, CA \\
\hline 12.6 & $11-07-84$ & 0 & -- & - & -- & -- & USGS, CA \\
\hline 20 & $11-07-84$ & 0 & -- & -- & -- & -- & USGS \\
\hline -- & -- & $\mathrm{H}$ & 15 & -- & -- & 1 & \\
\hline-- & -- & $\mathrm{H}$ & 8 & -- & -- & 1 & \\
\hline
\end{tabular}




\begin{tabular}{|c|c|c|c|c|c|c|c|c|c|c|c|}
\hline $\begin{array}{l}\text { Local } \\
\text { site } \\
\text { number }\end{array}$ & $\begin{array}{l}\text { Lati- } \\
\text { tude }\end{array}$ & $\begin{array}{l}\text { Long- } \\
\text { tude }\end{array}$ & Owner or user & $\begin{array}{l}\text { Year } \\
\text { comp- } \\
\text { leted }\end{array}$ & $\begin{array}{l}\text { Altitude } \\
\text { above } \\
\text { sea } \\
\text { level } \\
\text { (ft) }\end{array}$ & $\begin{array}{l}\text { Diam- } \\
\text { eter } \\
\text { of } \\
\text { well } \\
\text { (in.) }\end{array}$ & $\begin{array}{l}\text { Depth to } \\
\text { bottom } \\
\text { of } \\
\text { casing } \\
\text { (ft) }\end{array}$ & $\begin{array}{l}\text { Casing } \\
\text { mate- } \\
\text { rial }\end{array}$ & $\begin{array}{l}\text { Dype } \\
\text { of } \\
\text { fin- } \\
\text { ish }\end{array}$ & $\begin{array}{l}\text { Depth to } \\
\text { bottom } \\
\text { of } \\
\text { open } \\
\text { section } \\
\text { (ft) }\end{array}$ & $\begin{array}{l}\text { Type } \\
\text { of } \\
\text { site }\end{array}$ \\
\hline
\end{tabular}

Raymond--Continued

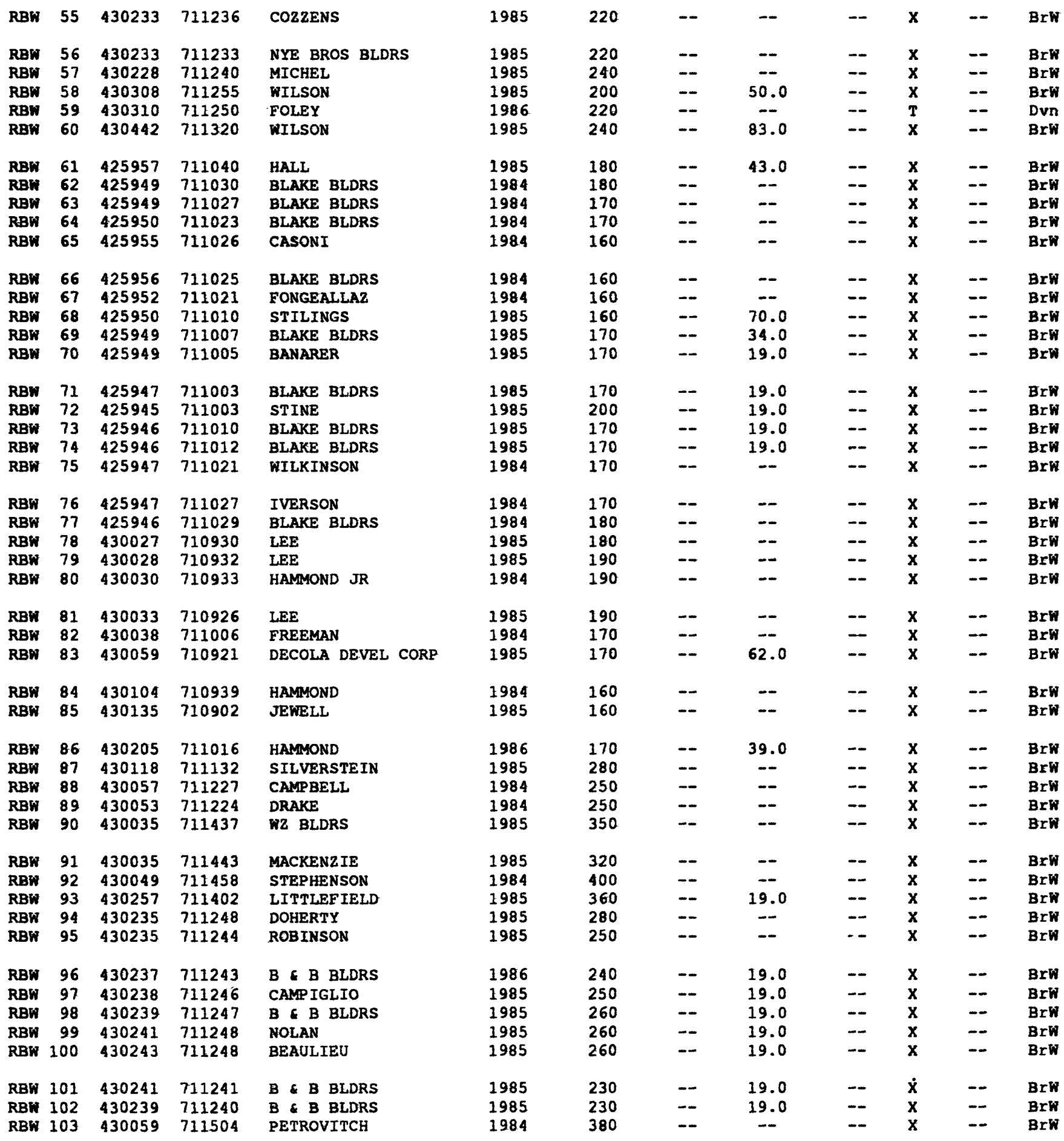


and springs--Continued

\begin{tabular}{|c|c|c|c|c|c|c|c|}
\hline Water & level & & $\begin{array}{l}\text { Maxi- } \\
\text { mum }\end{array}$ & $s$ & $\begin{array}{l}\text { Specific } \\
\text { capa- } \\
\text { city }\end{array}$ & 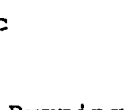 & \\
\hline
\end{tabular}

Raymond--Cont inued

\begin{tabular}{|c|c|c|c|c|c|c|c|}
\hline-- & -- & $\mathrm{H}$ & 10 & -- & -- & 1 & \\
\hline-- & -- & $\mathrm{H}$ & 15 & -- & -- & 1.5 & FLOWING WELL \\
\hline-- & -- & $\mathrm{H}$ & 12 & -- & -- & .5 & \\
\hline-- & -- & $\mathrm{H}$ & 6 & -- & -- & 1 & \\
\hline-- & -- & $\mathrm{H}$ & 15 & -- & -- & 2 & \\
\hline-- & -- & $\mathrm{H}$ & 2 & -- & -- & 1 & \\
\hline-- & -- & $\mathrm{H}$ & 15 & -- & -- & -- & \\
\hline-- & -- & $\mathrm{H}$ & 30 & -- & -- & & \\
\hline-- & -- & $\mathrm{H}$ & 10 & -- & -- & & \\
\hline-- & -- & $\mathrm{H}$ & 15 & -- & -- & .1 & \\
\hline-- & -- & $\mathrm{H}$ & 50 & -- & -- & & \\
\hline-- & -- & $\mathrm{H}$ & 50 & -- & -- & & \\
\hline-- & -- & $\mathrm{H}$ & 4 & -- & -- & .1 & \\
\hline- & -- & $\mathrm{H}$ & 4 & -- & -- & .2 & \\
\hline-- & -- & $\mathrm{H}$ & 5 & -- & -- & .1 & \\
\hline-- & -- & $\mathrm{H}$ & 10 & -- & -- & .2 & \\
\hline-- & -- & $\mathrm{H}$ & 10 & -- & -- & .1 & \\
\hline-- & -- & $\mathrm{H}$ & 10 & -- & -- & .1 & \\
\hline- & -- & $\mathrm{H}$ & 5 & -- & -- & .2 & \\
\hline-- & -- & $\mathrm{H}$ & 10 & -- & -- & .1 & \\
\hline-- & -- & $\mathrm{H}$ & 8 & -- & -- & .1 & \\
\hline-- & -- & $\mathrm{H}$ & 10 & -- & -- & .1 & \\
\hline-- & -- & $\mathrm{H}$ & 4 & -- & -- & .2 & \\
\hline-- & -- & $\mathrm{H}$ & 30 & -- & -- & 1 & \\
\hline-- & -- & $\mathrm{H}$ & 10 & -- & - & 2 & \\
\hline 23 & $04-26-84$ & $\mathrm{H}$ & 10 & -- & -- & -- & \\
\hline 18 & $05-27-85$ & $\mathrm{H}$ & 3 & -- & -- & 2 & \\
\hline-- & -- & $\mathrm{H}$ & 10 & -- & -- & .2 & \\
\hline-- & -- & $\mathrm{H}$ & 50 & -- & -- & .5 & \\
\hline 5 & $04-12-84$ & $\mathrm{H}$ & 20 & -- & -- & -- & \\
\hline-- & -- & $\mathrm{H}$ & 8 & -- & -- & -- & \\
\hline 10 & $05-13-86$ & $\mathrm{H}$ & 50 & -- & -- & .2 & \\
\hline-- & -- & $\mathrm{H}$ & 10 & -- & -- & 1 & \\
\hline-- & -- & $\mathrm{H}$ & 60 & $\cdots$ & -- & .1 & \\
\hline-- & -- & $\mathrm{H}$ & 25 & - & -- & -- & \\
\hline 40 & $07-31-85$ & $\mathrm{H}$ & 5 & -- & -- & 2 & \\
\hline-- & -- & $\mathrm{H}$ & 10 & -- & -- & .2 & \\
\hline-- & -- & $\mathrm{H}$ & 10 & -- & -- & -- & \\
\hline-- & -- & $\mathrm{H}$ & 12 & -- & -- & 1 & \\
\hline-- & -- & $\mathrm{H}$ & 10 & -- & -- & 1 & \\
\hline-- & -- & $\mathrm{H}$ & 15 & -- & -- & 2 & \\
\hline-- & -- & $\mathrm{H}$ & 20 & -- & -- & 1 & \\
\hline-- & -- & $\mathrm{H}$ & 6.5 & -- & -- & 1 & \\
\hline-- & -- & $\mathrm{H}$ & 20 & -- & -- & 1 & \\
\hline-- & -- & $\mathrm{H}$ & 25 & -- & -- & 1 & \\
\hline-- & -- & $\mathrm{H}$ & 7.5 & -- & -- & 1 & \\
\hline-- & -- & $\mathrm{H}$ & 25 & -- & -- & 1 & \\
\hline-- & -- & $\mathrm{H}$ & 3 & -- & -- & 1 & \\
\hline 20 & $10-04-84$ & $\mathrm{H}$ & 6 & -- & -- & 1 & \\
\hline
\end{tabular}




\begin{tabular}{|c|c|c|c|c|c|c|c|c|c|c|c|}
\hline $\begin{array}{l}\text { Local } \\
\text { site } \\
\text { number }\end{array}$ & $\begin{array}{l}\text { Lat1- } \\
\text { tude }\end{array}$ & $\begin{array}{l}\text { Long- } \\
\text { tude }\end{array}$ & Owner or user & $\begin{array}{l}\text { Year } \\
\text { comp- } \\
\text { leted }\end{array}$ & $\begin{array}{c}\text { Altitude } \\
\text { above } \\
\text { sea } \\
\text { level } \\
\text { (ft) }\end{array}$ & $\begin{array}{l}\text { Diam- } \\
\text { eter } \\
\text { of } \\
\text { well } \\
\text { (in.) }\end{array}$ & $\begin{array}{l}\text { Depth to } \\
\text { bottom } \\
\text { of } \\
\text { casing } \\
\text { (ft) }\end{array}$ & $\begin{array}{l}\text { Casing } \\
\text { mate- } \\
\text { rial }\end{array}$ & $\begin{array}{l}\text { Type } \\
\text { of } \\
\text { fin- } \\
\text { 1sh }\end{array}$ & $\begin{array}{l}\text { Depth to } \\
\text { bottom } \\
\text { of } \\
\text { open } \\
\text { section } \\
\text { (ft) }\end{array}$ & $\begin{array}{l}\text { Type } \\
\text { of } \\
\text { site }\end{array}$ \\
\hline
\end{tabular}

Raymond--Cont inued

\begin{abstract}
RBW $104 \quad 430057 \quad 711505$ HARVEY RBW 105430055711506 KINGMAN
\end{abstract}

1984

1985

370
370

$\overline{--}$

$--$
BrW BrW

\section{Sandown}

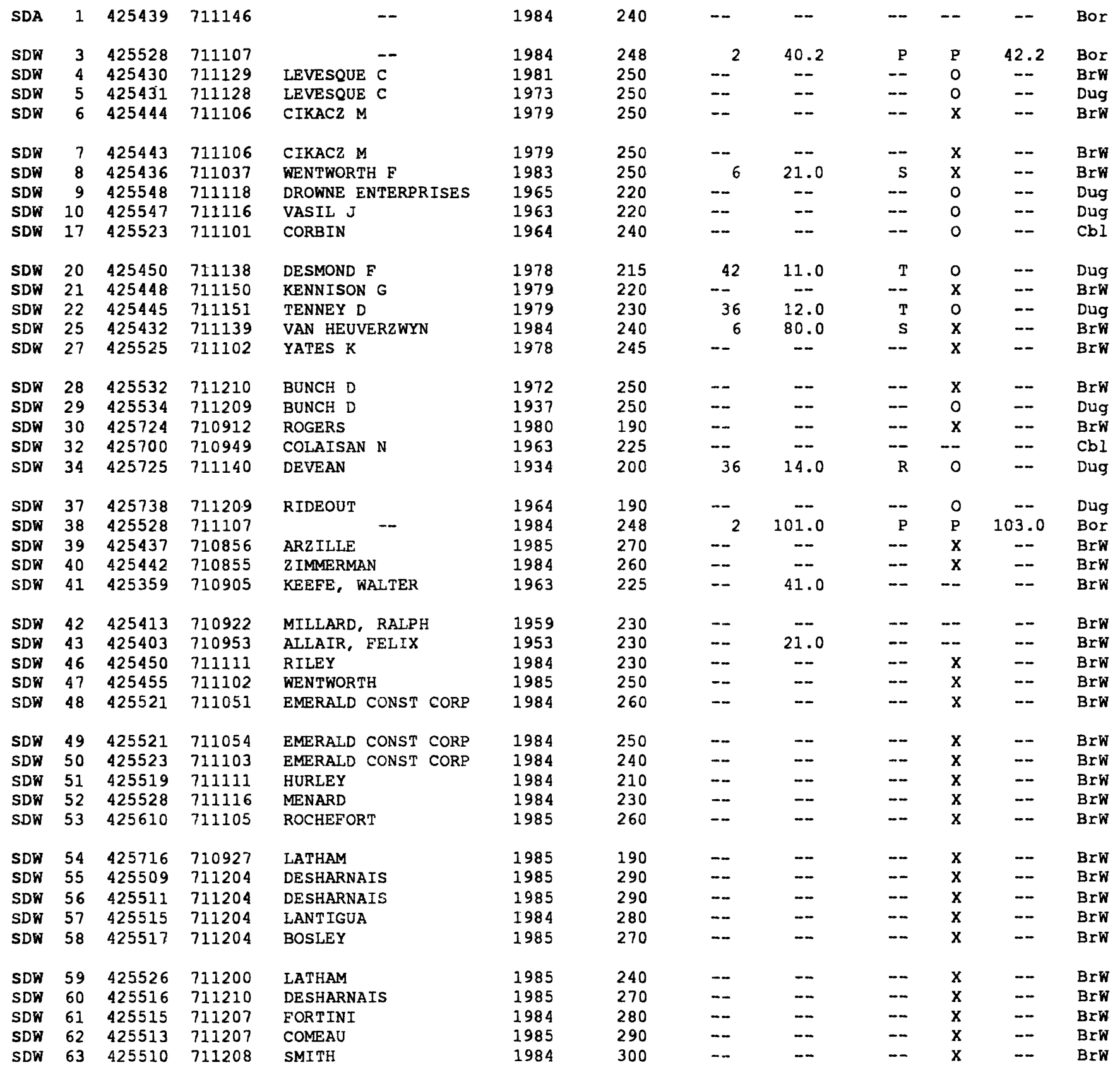




\begin{tabular}{|c|c|c|c|c|c|c|}
\hline Water & = level & & $\begin{array}{l}\text { Maxi- } \\
\text { mum } \\
\text { well }\end{array}$ & saw- & $\begin{array}{l}\text { Specific } \\
\text { capa- } \\
\text { city }\end{array}$ & \\
\hline $\begin{array}{l}\text { Depth } \\
\text { (ft) }\end{array}$ & $\begin{array}{c}\text { Date } \\
\text { (mm-dd-yy) }\end{array}$ & Use & $\begin{array}{c}\text { (gall } \\
\text { min) }\end{array}$ & $\begin{array}{l}\text { down } \\
(\mathrm{ft})\end{array}$ & $\begin{array}{l}\text { min)/ period } \\
\mathrm{ft}) \text { (hr) }\end{array}$ & Remarks \\
\hline
\end{tabular}

$\underline{\text { Raymond--Cont inued }}$

$\begin{array}{ccccccc}20 & 08-20-84 & \mathrm{H} & 8 & -- & -- & -- \\ -- & -- & \mathrm{H} & 20 & -- & -- & .2\end{array}$

Sandown

\begin{tabular}{|c|c|c|c|c|c|c|c|}
\hline-- & -- & $\mathrm{T}$ & -- & -- & -- & -- & USGS \\
\hline 31.2 & $12-17-84$ & 0 & -- & -- & -- & -- & USGS, CA \\
\hline 75 & $01-01-81$ & $\mathrm{H}$ & -- & -- & -- & -- & WATER TESTED BY WSPCC, SEDIMENT \\
\hline 8 & $01-01-73$ & $\mathrm{U}$ & -- & -- & -- & -- & WATER TESTED BY WSPCC, SEDIMENT \\
\hline-- & -- & $\mathrm{U}$ & -- & -- & -- & -- & DRY WELL \\
\hline-- & -- & $\mathrm{H}$ & -- & -- & -- & -- & \\
\hline-- & -- & $\mathrm{H}$ & -- & -- & -- & -- & \\
\hline 8.1 & $07-20-84$ & $\mathrm{~T}$ & -- & -- & -- & -- & REPORTED BY RENTER; POOR TASTE, IRON \\
\hline 1.5 & $01-01-63$ & $\mathrm{H}$ & -- & -- & -- & -- & \\
\hline-- & -- & $\mathrm{H}$ & -- & -- & -- & -- & FLOWING WELL \\
\hline 5.5 & $01-01-78$ & $\mathrm{H}$ & -- & -- & -- & -- & \\
\hline-- & -- & $\mathrm{H}$ & -- & -- & -- & -- & \\
\hline 7.5 & $01-01-79$ & $\mathrm{H}$ & -- & -- & -- & -- & \\
\hline-- & -- & $\mathrm{H}$ & -- & -- & -- & -- & \\
\hline-- & -- & $\mathrm{H}$ & -- & -- & -- & -- & \\
\hline-- & -- & $\mathrm{H}$ & -- & -- & -- & -- & WATER TESTED BY WSPCC, TRACE IRON \\
\hline-- & -- & $\mathrm{H}$ & -- & -- & -- & -- & WATER TESTED WSPCC, TRACE IRON \\
\hline-- & -- & $\mathrm{H}$ & -- & -- & -- & -- & \\
\hline-- & -- & $\mathrm{H}$ & -- & -- & -- & -- & WATER HAS HIGH IRON ACCORDING TO OWNER \\
\hline-- & -- & $\mathrm{U}$ & -- & -- & -- & -- & WATER TESTED BY WSPCC, TRACE IRON \\
\hline 11 & $01-01-64$ & $\mathrm{H}, \mathrm{T}$ & -- & -- & -- & -- & WATER TESTED BY WSPCC, TRACE IRON \\
\hline 32.2 & $12-17-84$ & 0 & -- & -- & -- & -- & USGS, CA \\
\hline 30 & $04-07-85$ & $\mathrm{H}$ & 5 & -- & -- & 4 & \\
\hline 15 & $06-01-84$ & $\mathrm{H}$ & 5 & -- & -- & 4 & \\
\hline 15 & $06--63$ & $\mathrm{H}$ & 6 & -- & -- & -- & \\
\hline 8 & $11--59$ & $\mathrm{H}$ & 6 & -- & -- & -- & \\
\hline 10 & $05-20-63$ & $\mathrm{H}$ & -- & -- & -- & -- & \\
\hline 5 & $10-30-84$ & $\mathrm{H}$ & 10 & -- & -- & 1 & \\
\hline-- & -- & $\mathrm{H}$ & 20 & -- & -- & 1 & \\
\hline 25 & $08-11-84$ & $\mathrm{H}$ & 5 & -- & -- & 4 & \\
\hline 16 & $07-13-84$ & $\mathrm{H}$ & 5 & -- & -- & 4 & \\
\hline-- & -- & $\mathrm{H}$ & 5 & -- & -- & 4 & \\
\hline 3 & $09-05-84$ & H & 5 & -- & -- & 4 & \\
\hline 30 & $01-17-84$ & $\mathrm{H}$ & 10 & -- & -- & .7 & \\
\hline 30 & $02-01-85$ & $\mathrm{H}$ & 5 & -- & -- & 4 & \\
\hline 20 & $08-22-85$ & $\mathrm{H}$ & 8 & -- & -- & .5 & \\
\hline 15 & $02-21-85$ & $\mathrm{H}$ & 8 & -- & -- & 1 & \\
\hline 25 & $05-08-85$ & $\mathrm{H}$ & 10 & -- & -- & 2 & \\
\hline-- & -- & $\mathrm{H}$ & 10 & -- & -- & 1 & \\
\hline 25 & $02-20-85$ & $\mathrm{H}$ & 25 & -- & -- & 1 & \\
\hline 20 & $05-06-85$ & $\mathrm{H}$ & 5 & -- & -- & 4 & \\
\hline 25 & $05-09-85$ & $\mathrm{H}$ & 4 & -- & -- & 1 & \\
\hline 18 & $10-12-84$ & $\mathrm{H}$ & 7 & -- & -- & 1 & \\
\hline 20 & $02-26-85$ & $\mathrm{H}$ & 10 & -- & -- & 1 & \\
\hline- & - & $\mathrm{H}$ & 100 & -- & -- & .5 & \\
\hline
\end{tabular}




\begin{tabular}{|c|c|c|c|c|c|c|c|c|c|c|c|}
\hline $\begin{array}{l}\text { Local } \\
\text { site } \\
\text { number }\end{array}$ & $\begin{array}{l}\text { Lati- } \\
\text { tude }\end{array}$ & $\begin{array}{l}\text { Long- } \\
\text { tude }\end{array}$ & Owner or user & $\begin{array}{l}\text { Year } \\
\text { comp- } \\
\text { leted }\end{array}$ & $\begin{array}{l}\text { Altitude } \\
\text { above } \\
\text { sea } \\
\text { level } \\
\text { (ft) }\end{array}$ & $\begin{array}{l}\text { Diam- } \\
\text { eter } \\
\text { of } \\
\text { well } \\
\text { (in.) }\end{array}$ & $\begin{array}{l}\text { Depth to } \\
\text { bottom } \\
\text { of } \\
\text { casing } \\
\text { (ft) }\end{array}$ & $\begin{array}{l}\text { Casing } \\
\text { mate- } \\
\text { rtal }\end{array}$ & $\begin{array}{l}\text { Type } \\
\text { of } \\
\text { fin- } \\
\text { ish }\end{array}$ & $\begin{array}{l}\text { epth to } \\
\text { bottom } \\
\text { of } \\
\text { open } \\
\text { section } \\
\text { (ft) }\end{array}$ & $\begin{array}{l}\text { Type } \\
\text { of } \\
\text { site }\end{array}$ \\
\hline
\end{tabular}

Sandown--Cont inued

\begin{tabular}{|c|c|c|c|c|c|c|c|c|c|c|c|c|}
\hline SDW & 64 & 425511 & 711217 & TIMELY BLDRS & 1984 & 300 & -- & -- & -- & $\mathrm{x}$ & - & Brh \\
\hline SDW & 65 & 425604 & 711251 & SULLIVAN & 1984 & 270 & -- & -- & -- & $\mathrm{x}$ & -- & Brh \\
\hline SDW & 66 & 425735 & 711223 & HIGGINS & 1984 & 220 & -- & -- & -- & $\mathrm{x}$ & -- & $\mathrm{Brh}$ \\
\hline SDW & 67 & 425735 & 711225 & HIGGINS & 1984 & 220 & -- & -- & $\rightarrow$ & $\mathrm{x}$ & - & Brh \\
\hline SDW & 68 & 425519 & 711131 & BARTLETT & 1985 & 220 & -- & -- & -- & $x$ & -- & Brh \\
\hline SDW & 69 & 425454 & 711251 & GIAQUINTA & 1984 & 420 & -- & -- & -- & $\mathrm{x}$ & -- & $\mathrm{Brh}$ \\
\hline SDW & 70 & 425723 & 711142 & EMERALD CONST CORP & 1984 & 210 & -- & -- & -- & $\mathrm{x}$ & - & $\mathrm{Brh}$ \\
\hline SDW & 71 & 425722 & 711137 & GIARD & 1985 & 200 & -- & -- & -- & $\mathrm{x}$ & -- & Brh \\
\hline SDW & 72 & 425723 & 711145 & RIOUX & 1984 & 210 & -- & -- & -- & $\mathrm{x}$ & -- & Brh \\
\hline
\end{tabular}

\begin{tabular}{|c|c|c|c|c|c|c|c|c|c|c|c|c|}
\hline SSB & 1 & 425932 & 705537 & NHDOT & 1957 & 42 & -- & -- & -- & -- & -- & Wsh \\
\hline SSB & 2 & 430000 & 705623 & NHDOT & 1958 & 2.0 & -- & -- & -- & -- & -- & Wsh \\
\hline SSW & 1 & 430121 & 705307 & CHICK $\mathrm{F} \mathrm{C}$ & 1952 & 140 & 36 & -- & $T$ & -- & -- & Dug \\
\hline SSW & 2 & $4302: 5$ & 705410 & WHITTAKER ARNOLO & 1941 & 130 & 6 & -- & $s$ & -- & -- & BrW \\
\hline SSW & 3 & 430227 & 705400 & EATON L M & -- & 130 & 36 & -- & R & -- & - & Dug \\
\hline SSW & 4 & 430013 & 705502 & SEAMMON LESLIE & -- & 90 & 40 & -- & $\mathbf{R}$ & -- & -- & Dug \\
\hline SSW & 5 & 430113 & 705348 & WIGGIN ERNEST JR & 1955 & 130 & 48 & -- & C & -- & -- & Dug \\
\hline SsW & 6 & 430044 & 705504 & CUSHMAN A B & - & 130 & 36 & -- & $\mathbf{R}$ & -- & - & \\
\hline SSW & 7 & 430226 & 705333 & STRATHAM, TOWN OF & 1935 & 170 & 36 & -- & $\mathbf{R}$ & -- & -- & \\
\hline SSW & 8 & 425921 & 705454 & AMSDEN RAIPH & 1934 & 160 & 60 & -- & $\mathbf{R}$ & -- & -- & Dug \\
\hline SSW & 9 & 425958 & 705330 & CHASE M S & -- & 130 & 40 & $\rightarrow$ & $\mathbf{R}$ & -- & -- & Dug \\
\hline SSW & 10 & 425914 & 705512 & EXETER, TOWN OF & 1929 & 60 & 300 & -- & $\mathrm{T}$ & -- & -- & Di \\
\hline SSW & 12 & 425915 & 705433 & STURGIS ESTATE & 1914 & 170 & 6 & -- & $s$ & -- & -- & \\
\hline SSW & 13 & 430012 & 705356 & LALOUD L F & 1939 & 140 & 6 & -- & $\mathbf{s}$ & -- & -- & BrW \\
\hline SSW & 14 & 430142 & 705440 & GREENWOOD CEM & 1937 & 70 & 6 & -- & $\mathbf{s}$ & -- & -- & BrW \\
\hline SSW & 15 & 430200 & 705544 & STRATHAM CHRISTIE & 1926 & 40 & 6 & -- & $\mathrm{s}$ & -- & -- & \\
\hline SSW & 16 & 430059 & 705313 & JEWELL SADIE \& HAR & $\Gamma 1933$ & 130 & 8 & -- & $\mathbf{s}$ & -- & -- & BrW \\
\hline SSW & 17 & 430049 & 705257 & LANGELIER VALMORE & 1955 & 110 & 6 & -- & $\mathbf{S}$ & -- & - & BrW \\
\hline SSW & 18 & 425914 & 705512 & EXETER, TOWN OE & 1955 & 60 & 2 & -- & $S$ & -- & $\rightarrow$ & Wsh \\
\hline SSW & 19 & 425938 & 705317 & USCOE & 1951 & 140 & 2 & -- & $\mathbf{s}$ & -- & -- & Ws \\
\hline SSW & 22 & 430041 & 705438 & USCOE & 1977 & 100 & -- & -- & -- & $\mathrm{P}$ & -- & \\
\hline SSW & 23 & 430045 & 705433 & USCOE & 1977 & 80 & -- & -- & $\rightarrow$ & $\mathrm{p}$ & -- & \\
\hline SSW & 24 & 430041 & 705435 & USCOE & 1977 & 100 & -- & -- & $-\infty$ & $\mathrm{p}$ & $-\infty$ & Ws \\
\hline SSW & 25 & 430044 & 705428 & USCOE & 1977 & 70 & -- & -- & -- & -- & -- & \\
\hline SSW & 26 & 430045 & 705406 & USCOE & 1977 & 60 & -- & -- & -- & $\mathrm{P}$ & -- & Wsh \\
\hline SSW & 27 & 430024 & 705357 & USCOE & 1977 & 120 & -- & -- & -- & $\mathrm{P}$ & -- & Wsh \\
\hline SSW & 28 & 430036 & 705342 & USCOE & 1977 & 80 & -- & -- & -- & $\mathbf{p}$ & - & \\
\hline SSW & 29 & 430021 & 705413 & USCOE & 1977 & 120 & -- & -- & -- & -- & -- & W: \\
\hline SSW & 30 & 430015 & 705432 & PRATT & 1984 & 100 & -- & -- & -- & $\mathrm{x}$ & - & \\
\hline SSW & 31 & 430017 & 705431 & ABRAMI & 1984 & 100 & -- & -- & -- & $\mathrm{X}$ & -- & BrW \\
\hline SSW & 32 & 430021 & 705351 & CARBONNEAU & 1984 & 110 & -- & -- & -- & $\mathrm{x}$ & -- & BrW \\
\hline SSW & 33 & 430020 & 705428 & SIMONSON & 1984 & 100 & -- & -- & -- & $\mathrm{x}$ & -- & Brw \\
\hline SSW & 34 & 430022 & 705427 & MALASKY BLDRS & 1984 & 120 & -- & -- & -- & $\mathrm{x}$ & -- & \\
\hline SSW & 35 & 430018 & 705438 & LABRUN & 1985 & 100 & -- & -- & $-\infty$ & $\mathrm{x}$ & -- & BrW \\
\hline SSW & 36 & 430018 & 705435 & HURLBERT & 1984 & 100 & -- & -- & -- & $\mathrm{x}$ & -- & $\mathrm{Br}$ \\
\hline SSW & 38 & 430020 & 705433 & BLAKE & 1984 & 100 & -- & -- & -- & $\mathrm{x}$ & -- & \\
\hline SSW & 39 & 430004 & 705519 & SULLIVAN TIRE & 1984 & 80 & -- & -- & $\rightarrow$ & $\mathrm{X}$ & -- & D \\
\hline SSW & 40 & 430023 & 705514 & STRATHAM AGWAY & 1985 & 80 & -- & -- & -- & $\mathrm{x}$ & - & \\
\hline
\end{tabular}




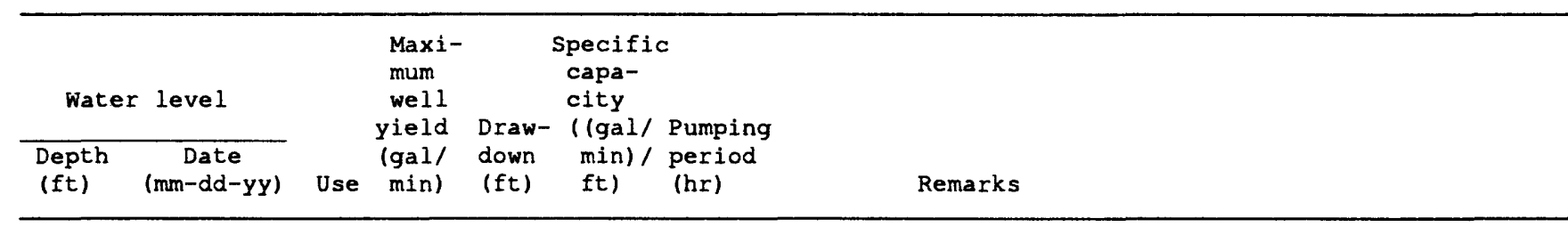

Sandown--Cont inued

$\begin{array}{lcrrrrr}-- & -- & \text { H } & 12 & -- & -- & 2.5 \\ 40 & 10-04-84 & \text { H } & 5 & -- & -- & 4 \\ 15 & 06-17-84 & \text { H } & 100 & -- & -- & .5 \\ 15 & 06-19-84 & \text { H } & 200 & -- & -- & .5 \\ 10 & 05-16-85 & \text { H } & 8 & -- & -- & 1 \\ 15 & & & & & & \\ 20 & 11-23-84 & \text { H } & 8 & -- & -- & 1 \\ -- & 09-23-84 & \text { H } & 5 & -- & -- & 4 \\ 12 & -- & \text { H } & 4 & -- & -- & .7\end{array}$

\section{Stratham}

\begin{tabular}{|c|c|c|c|c|c|c|}
\hline-- & -- & $T$ & -- & -- & -- & -- \\
\hline-- & -- & $T$ & -- & -- & -- & -- \\
\hline 15.3 & $06-22-54$ & $\mathrm{H}$ & -- & -- & -- & -- \\
\hline-- & -- & $\mathrm{H}$ & 10 & -- & -- & -- \\
\hline 10.4 & $09-06-55$ & $\mathrm{H}$ & -- & -- & -- & -- \\
\hline 12.3 & $09-09-55$ & $\mathrm{H}$ & -- & -- & -- & -- \\
\hline 9.4 & $09-09-55$ & $\mathrm{H}$ & -- & -- & -- & -- \\
\hline 16.8 & $09-15-55$ & $\mathrm{H}$ & -- & -- & - & -- \\
\hline 15.9 & $09-13-55$ & P & -- & -- & -- & -- \\
\hline 8 & $09-14-55$ & $\mathrm{H}$ & 8 & -- & -- & -- \\
\hline 12 & $09-14-55$ & $\mathrm{H}$ & -- & -- & -- & -- \\
\hline-- & -- & $P$ & -- & -- & -- & -- \\
\hline-- & -- & $\mathrm{H}$ & 15 & -- & - & -- \\
\hline-- & -- & H & 7 & -- & -- & -- \\
\hline-- & -- & I & 100 & -- & -- & -- \\
\hline-- & -- & $\mathrm{H}$ & -- & -- & -- & -- \\
\hline-- & -- & $\mathrm{H}$ & 6 & -- & -- & -- \\
\hline 16.8 & $09-09-55$ & $\mathrm{H}$ & -- & -- & -- & -- \\
\hline- & -- & 0 & -- & -- & -- & -- \\
\hline-- & -- & 0 & -- & -- & -- & -- \\
\hline 13.6 & $10-18-77$ & 0 & 60 & -- & -- & 2 \\
\hline 11.3 & $10-19-77$ & 0 & 5 & -- & -- & 1 \\
\hline 10.5 & $06-01-77$ & 0 & 40 & -- & -- & 1 \\
\hline-- & - & 0 & -- & -- & -- & -- \\
\hline-- & -- & 0 & -- & -- & -- & -- \\
\hline- & -- & 0 & -- & -- & -- & -- \\
\hline-- & -- & 0 & -- & -- & -- & -- \\
\hline-- & -- & 0 & -- & -- & -- & -- \\
\hline-- & -- & $\mathrm{H}$ & 50 & -- & -- & -- \\
\hline-- & -- & $\mathrm{H}$ & 20 & -- & -- & -- \\
\hline-- & -- & $\mathrm{H}$ & 9 & -- & -- & -- \\
\hline 10 & $04-30-84$ & $\mathrm{H}$ & 10 & -- & -- & .2 \\
\hline-- & -- & $\mathrm{H}$ & 6 & -- & -- & .1 \\
\hline-- & -- & $\mathrm{H}$ & 10 & -- & -- & -- \\
\hline 12 & $04-10-84$ & $\mathrm{H}$ & 20 & -- & -- & .1 \\
\hline-- & -- & $\mathrm{H}$ & 30 & -- & -- & -- \\
\hline-- & -- & $\dot{c}$ & 8.5 & -- & -- & 1 \\
\hline - & -- & $\mathrm{H}$ & 20 & -- & -- & - \\
\hline
\end{tabular}

BATTERY OF 9 WELLS: 6 30-IN. DIA.; 2 52-INCH DIA.; 1 300-IN. DIA.; LOG FOR SSW-18 AT SAME LOCATION

NO LOG FROM 28-29 FEET

NO LOG FROM 21-24 FEET

AT SAME LOCATION AS SSW 10 
Table 2.--Description of wells, borings,

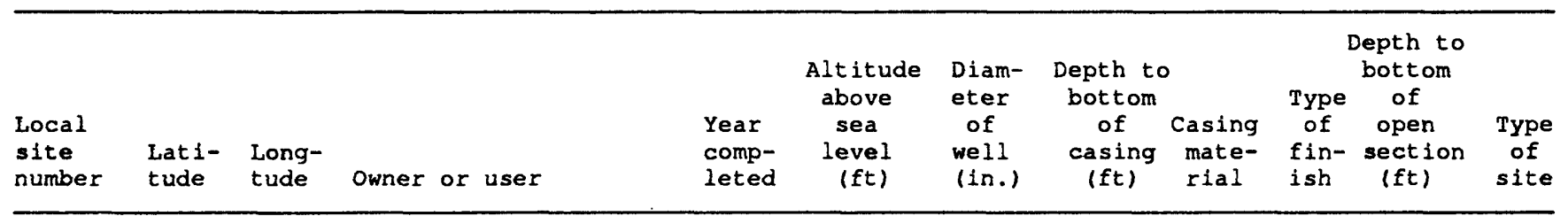

Stratham--Cont inued

\begin{tabular}{|c|c|c|c|c|c|c|c|c|c|c|c|c|}
\hline ssw & 41 & 430104 & 705527 & DAVIS & 1984 & 40 & -- & -- & -- & $x$ & -- & BrW \\
\hline SSW & 42 & 430108 & 705527 & DELTA TRIANGLE & 1985 & 40 & -- & - & -- & $x$ & -- & BrW \\
\hline ssw & 43 & 430112 & 705522 & SWALE CORP & 1984 & 60 & -- & -- & -- & $\mathbf{x}$ & -- & BrW \\
\hline SSW & 44 & 430120 & 705519 & EMANUEL CO INC & 1984 & 60 & -- & -- & -- & $\mathrm{x}$ & -- & BrW \\
\hline SSW & 45 & 430120 & 705525 & EMANUEL & 1984 & 60 & -- & -- & -- & $x$ & -- & BrW \\
\hline SSW & 46 & 430111 & 705528 & SWALE CORP & 1984 & 40 & -- & -- & -- & $\mathbf{x}$ & -- & $\mathrm{BrW}$ \\
\hline SSW & 47 & 430112 & 705536 & WINDING BROOK CORP & 1985 & 30 & -- & -- & -- & $\mathrm{x}$ & -- & $\mathrm{BrW}$ \\
\hline SSW & 48 & 430113 & 705537 & WINDING BROOK CORP & 1985 & 30 & -- & -- & -- & $x$ & -- & $\mathrm{BrW}$ \\
\hline SSW & 49 & 430233 & 705432 & $M \& J$ BLDRS & 1984 & 50 & -- & -- & -- & $x$ & -- & $\mathrm{BrW}$ \\
\hline SSW & 50 & 430232 & 705430 & $M \& J$ BLDRS & 1984 & 50 & -- & -- & -- & -- & -- & BrW \\
\hline ssw & 52 & 430229 & 705428 & $M \notin J$ BLDRS & 1984 & 60 & -- & -- & -- & $\mathrm{x}$ & -- & BrW \\
\hline ssw & 53 & 430232 & 705426 & $M \& J$ BLDRS & 1984 & 60 & -- & -- & -- & $x$ & -- & BrW \\
\hline ssw & 55 & 430237 & 705354 & HAMEL & 1985 & 120 & -- & -- & -- & $\mathrm{x}$ & -- & BrW \\
\hline SSW & 56 & 430238 & 705348 & MCFARLAND & 1985 & 120 & -- & -- & -- & $\mathbf{x}$ & -- & BrW \\
\hline ssw & 60 & 430247 & 705338 & GLENGARRY CONDO & 1985 & 100 & -- & -- & -- & $x$ & -- & BrW \\
\hline ssw & 61 & 430247 & 705339 & GLENGARRY CONDO & 1985 & 100 & -- & -- & -- & $x$ & -- & BrW \\
\hline SSW & 62 & 430230 & 705424 & $M \& J$ BLDRS & 1984 & 70 & -- & -- & -- & $\mathbf{x}$ & -- & $\mathrm{BrW}$ \\
\hline SSW & 63 & 430239 & 705351 & SWALE CORP & 1985 & 120 & -- & -- & -- & $\mathrm{x}$ & -- & Brw \\
\hline SsW & 64 & 430246 & 705348 & SCHWARTZ & 1984 & 100 & -- & -- & -- & $\mathbf{x}$ & -- & BrW \\
\hline SSW & 65 & 430248 & 705336 & GLENGARRY CONDO & 1985 & 100 & -- & -- & -- & $\mathrm{x}$ & -- & $\mathrm{BrW}$ \\
\hline ssw & 66 & 430247 & 705404 & DABNEY & 1984 & 80 & -- & -- & -- & $x$ & -- & Brw \\
\hline Ssw & 67 & 430304 & 705349 & EROIS CONST & 1984 & 60 & -- & -- & -- & $x$ & -- & $\mathrm{BrW}$ \\
\hline SSW & 68 & 430308 & 705402 & WOOD & 1984 & 50 & -- & -- & -- & $\mathrm{X}$ & -- & BrW \\
\hline SSW & 69 & 430308 & 705407 & THIBODEAU & 1984 & 40 & -- & -- & -- & $x$ & -- & BrW \\
\hline ssw & 70 & 430308 & 705413 & SADUR & 1984 & 20 & -- & -- & -- & $x$ & -- & $\mathrm{BrW}$ \\
\hline SSW & 71 & 430311 & 705413 & PHOENIX & 1984 & 20 & -- & -- & -- & $\mathrm{x}$ & -- & BrW \\
\hline ssw & 72 & 430311 & 705409 & FARINA & 1984 & 30 & -- & -- & -- & $\mathbf{x}$ & -- & BrW \\
\hline SSW & 73 & 430311 & 705405 & DRAKE & 1984 & 30 & -- & -- & -- & $x$ & -- & $\mathrm{BrW}$ \\
\hline SSW & 74 & 425911 & 705329 & COLE & 1984 & 120 & -- & - & -- & $x$ & -- & BrW \\
\hline SSW & 75 & 425919 & 705337 & $M \& J$ BLDRS & 1984 & 120 & -- & -- & -- & $\mathrm{x}$ & -- & BrW \\
\hline SSW & 76 & 425922 & 705337 & TIZZARD & 1984 & 100 & -- & -- & -- & $\mathrm{x}$ & -- & BrW \\
\hline ssw & 78 & 425921 & 705320 & KIRTLAND & 1985 & 120 & -- & -- & -- & $x$ & -- & $\mathrm{BrW}$ \\
\hline SSW & 79 & 425929 & 705254 & KROOSS & 1984 & 80 & -- & -- & -- & $\mathrm{x}$ & -- & BrW \\
\hline SSW & 80 & 425938 & 705302 & ERICK & 1984 & 100 & -- & -- & -- & $\mathrm{x}$ & -- & BrW \\
\hline SSW & 81 & 425928 & 705312 & CHAPPMAN & 1984 & 120 & -- & -- & -- & $x$ & -- & BrW \\
\hline SSW & 82 & 425926 & 705315 & MACKENZIE & 1985 & 120 & -- & -- & -- & $\mathrm{x}$ & - & BrW \\
\hline SSW & 83 & 425925 & 705317 & MERCHANT & 1985 & 120 & -- & -- & -- & $\mathrm{x}$ & -- & BrW \\
\hline SSW & 84 & 425924 & 705320 & SCAMMON & 1985 & 120 & -- & -- & -- & $\mathrm{x}$ & -- & BrW \\
\hline SSW & 85 & 425927 & 705325 & HENRY M CONST & 1985 & 100 & -- & -- & -- & $\mathbf{x}$ & -- & BrW \\
\hline SSW & 86 & 425930 & 705324 & $M \& J$ BLDRS & 1984 & 120 & -- & -- & -- & $x$ & -- & $\mathrm{BrW}$ \\
\hline SSW & 87 & 425930 & 705320 & SCAMMON & 1985 & 120 & -- & -- & -- & $x$ & -- & BrW \\
\hline SSW & 88 & 425932 & 705308 & CHAPMAN & 1985 & 120 & -- & -- & -- & $x$ & -- & BrW \\
\hline SSW & 89 & 425937 & 705314 & $M \& J$ BLDRS & 1984 & 140 & -- & -- & -- & $\mathrm{x}$ & -- & BrW \\
\hline SSW & 90 & 425939 & 705316 & -- & -- & 140 & -- & -- & -- & $\mathbf{x}$ & $-\infty$ & Brw \\
\hline SSW & 91 & 425936 & 705324 & $M \subset J$ BLDRS & 1984 & 120 & -- & -- & -- & $\mathrm{x}$ & -- & Brw \\
\hline SSW & 92 & 425938 & 705324 & BARTLEY & 1985 & 120 & -- & -- & -- & $\mathrm{x}$ & -- & BrW \\
\hline SSW & 93 & 425943 & 705320 & HENRY M CONST & 1985 & 140 & -- & -- & -- & $\mathbf{x}$ & -- & BrW \\
\hline ssw & 94 & 425946 & 705319 & DEVELCO INC & 1984 & 120 & -- & -- & -- & $x$ & -- & $\mathrm{BrW}$ \\
\hline SSW & 95 & 425947 & 705320 & DEVELCO INC & 1984 & 120 & -- & -- & -- & $x$ & $-\infty$ & BrW \\
\hline SSW & 96 & 425949 & 705322 & DEVELCO INC & 1985 & 120 & -- & -- & -- & $\mathbf{x}$ & -- & BrW \\
\hline
\end{tabular}


and springs--Continued

\begin{tabular}{|c|c|c|c|c|c|c|c|}
\hline Water & C level & & $\begin{array}{l}\text { Maxi- } \\
\text { mum } \\
\text { well }\end{array}$ & 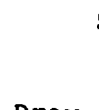 & $\begin{array}{l}\text { Specific } \\
\text { capa- } \\
\text { city }\end{array}$ & c & \\
\hline
\end{tabular}

Stratham--Continued

\begin{tabular}{|c|c|c|c|c|c|c|c|}
\hline-- & -- & $\mathrm{H}$ & 20 & -- & -- & -- & \\
\hline-- & -- & $\mathrm{H}$ & -- & -- & -- & -- & FLOWING WELL \\
\hline- & -- & $\mathrm{H}$ & 5 & -- & -- & -- & \\
\hline-- & -- & $\mathrm{H}$ & 3 & -- & -- & -- & \\
\hline-- & -- & $\mathrm{H}$ & 3 & -- & -- & -- & \\
\hline-- & -- & $\mathrm{H}$ & 8 & -- & -- & -- & \\
\hline-- & -- & $\mathrm{H}$ & 50 & -- & -- & -- & \\
\hline-- & -- & H & 50 & -- & -- & -- & \\
\hline-- & -- & $\mathrm{H}$ & 8 & -- & -- & -- & \\
\hline-- & -- & $\mathrm{H}$ & 15 & -- & -- & -- & \\
\hline-- & -- & H & 20 & -- & -- & -- & \\
\hline-- & -- & H & 25 & -- & -- & -- & \\
\hline-- & -- & $\mathrm{H}$ & 6 & -- & -- & 1.5 & \\
\hline-- & -- & H & 20 & -- & -- & -- & \\
\hline-- & -- & $\mathrm{H}$ & 30 & -- & -- & -- & \\
\hline-- & -- & $\mathrm{H}$ & 25 & -- & -- & -- & \\
\hline-- & -- & $\mathrm{H}$ & 75 & -- & -- & -- & \\
\hline-- & -- & $\mathrm{H}$ & 25 & -- & -- & -- & \\
\hline- & -- & $\mathrm{H}$ & 4 & -- & -- & -- & \\
\hline-- & -- & $\mathrm{H}$ & 30 & -- & -- & -- & \\
\hline-- & -- & $\mathrm{H}$ & 50 & -- & -- & -- & \\
\hline-- & -- & -- & 10 & -- & -- & -- & \\
\hline-- & -- & $\mathrm{H}$ & 20 & -- & -- & -- & \\
\hline-- & -- & H & 15 & -- & -- & -- & \\
\hline-- & -- & $\mathrm{H}$ & 10 & -- & -- & -- & \\
\hline-- & -- & $\mathrm{H}$ & 75 & -- & -- & -- & \\
\hline-- & -- & $\mathrm{H}$ & 10 & -- & -- & -- & \\
\hline-- & -- & H & 20 & -- & -- & -- & \\
\hline-- & -- & $\mathrm{H}$ & 10 & -- & -- & -- & \\
\hline-- & -- & -- & 10 & -- & -- & -- & \\
\hline-- & -- & $\mathrm{H}$ & 5 & -- & -- & -- & \\
\hline-- & -- & H & 8 & -- & -- & -- & \\
\hline-- & -- & $\mathrm{H}$ & 15 & -- & -- & -- & \\
\hline-- & -- & $\mathrm{H}$ & 10 & -- & -- & -- & \\
\hline 25 & $12-08-84$ & $\mathrm{H}$ & 75 & -- & -- & 1 & \\
\hline-- & -- & $\mathrm{H}$ & 5 & -- & -- & -- & \\
\hline-- & -- & $\mathrm{H}$ & 50 & -- & -- & -- & \\
\hline-- & -- & $\mathrm{H}$ & 12 & -- & -- & -- & \\
\hline -- & -- & $\mathrm{H}$ & 4 & -- & -- & -- & \\
\hline-- & -- & $\mathrm{H}$ & 50 & -- & -- & -- & \\
\hline-- & -- & $\mathrm{H}$ & 8 & -- & -- & -- & \\
\hline-- & -- & H & 8 & -- & -- & 1 & \\
\hline-- & -- & $\mathrm{H}$ & 6 & -- & -- & -- & \\
\hline-- & -- & H & -- & -- & -- & -- & \\
\hline-- & -- & $\mathrm{H}$ & 7 & -- & -- & -- & \\
\hline-- & -- & H & 1.5 & -- & -- & -- & \\
\hline-- & -- & $\mathrm{H}$ & 3 & -- & -- & -- & \\
\hline-- & -- & $\mathrm{H}$ & 20 & -- & -- & -- & \\
\hline-- & -- & H & -- & -- & -- & -- & \\
\hline-- & -- & H & 15 & -- & -- & -- & \\
\hline
\end{tabular}


Table 2.-Description of wells, borings,

\begin{tabular}{|c|c|c|c|c|c|c|c|c|c|c|c|}
\hline $\begin{array}{l}\text { Local } \\
\text { site } \\
\text { number }\end{array}$ & $\begin{array}{l}\text { Lat1- } \\
\text { tude }\end{array}$ & $\begin{array}{l}\text { Long- } \\
\text { tude }\end{array}$ & Owner or user & $\begin{array}{l}\text { Year } \\
\text { comp- } \\
\text { leted }\end{array}$ & $\begin{array}{l}\text { Altitude } \\
\text { above } \\
\text { sea } \\
\text { level } \\
\text { (ft) }\end{array}$ & $\begin{array}{l}\text { Diam- } \\
\text { eter } \\
\text { of } \\
\text { well } \\
\text { (in.) }\end{array}$ & $\begin{array}{l}\text { Depth to } \\
\text { bottom } \\
\text { of } \\
\text { casing } \\
\text { (ft) }\end{array}$ & $\begin{array}{l}\text { Casing } \\
\text { mate- } \\
\text { rlal }\end{array}$ & $\begin{array}{l}\text { Type } \\
\text { of } \\
\text { fin- } \\
\text { ish }\end{array}$ & $\begin{array}{l}\text { Depth to } \\
\text { bottom } \\
\text { of } \\
\text { open } \\
\text { section } \\
\text { (ft) }\end{array}$ & $\begin{array}{l}\text { Type } \\
\text { of } \\
\text { site }\end{array}$ \\
\hline \multicolumn{12}{|c|}{ Stratham--Continued } \\
\hline $\begin{array}{ll}\text { SSW } & 100 \\
\text { SSW } 101 \\
\text { SSW } 102 \\
\text { SSW } 103 \\
\text { SSW } 104\end{array}$ & $\begin{array}{l}425946 \\
425944 \\
425927 \\
425923 \\
425959\end{array}$ & $\begin{array}{l}705315 \\
705316 \\
705450 \\
705455 \\
705524\end{array}$ & $\begin{array}{l}\text { DEVELCO OF STRATHAM } \\
\text { DEVELCO INC } \\
\text { NEMO } \\
\text { DERWIECKI } \\
\text { DEMOULAS SUPERMARKET }\end{array}$ & $\begin{array}{l}1984 \\
1985 \\
1984 \\
1984 \\
1985\end{array}$ & $\begin{array}{r}120 \\
120 \\
160 \\
160 \\
60\end{array}$ & $\begin{array}{l}-- \\
-- \\
-- \\
--\end{array}$ & $\begin{array}{l}-- \\
-- \\
-- \\
--\end{array}$ & $\begin{array}{l}-- \\
-- \\
-- \\
-\end{array}$ & $\begin{array}{l}\mathbf{x} \\
\mathbf{x} \\
\mathbf{x} \\
\mathbf{x} \\
\mathbf{x}\end{array}$ & $\begin{array}{l}-- \\
-- \\
-- \\
--\end{array}$ & $\begin{array}{l}\text { BrW } \\
\text { BrW } \\
\text { BrW } \\
\text { BrW } \\
\text { BrW }\end{array}$ \\
\hline $\begin{array}{ll}\text { ssw } & 110 \\
\text { ssw } & 112 \\
\text { ssw } & 113 \\
\text { ssw } & 114 \\
\text { ssw } & 115\end{array}$ & $\begin{array}{l}425910 \\
430001 \\
430228 \\
430041 \\
425954\end{array}$ & $\begin{array}{l}705324 \\
705356 \\
705423 \\
705504 \\
705401\end{array}$ & $\begin{array}{l}\text { GOODRIDGE JR } \\
\text { KIRRIEMUIR CONDOS } \\
\text { M J BLDRS } \\
\text { EXETER AREA GROUP HME } \\
\text { ORCHARD HOMES }\end{array}$ & $\begin{array}{l}1985 \\
1984 \\
1984 \\
1985 \\
1984\end{array}$ & $\begin{array}{r}100 \\
80 \\
70 \\
120 \\
80\end{array}$ & $\begin{array}{l}-- \\
-- \\
-- \\
--\end{array}$ & $\begin{array}{l}-- \\
-- \\
-- \\
--\end{array}$ & $\begin{array}{l}-- \\
-- \\
--\end{array}$ & $\begin{array}{l}\mathbf{x} \\
\mathbf{x} \\
\mathbf{x} \\
\mathbf{x} \\
\mathbf{x}\end{array}$ & $\begin{array}{l}-- \\
-- \\
-- \\
--\end{array}$ & $\begin{array}{l}\text { BrW } \\
\text { BrW } \\
\text { BrW } \\
\text { BrW } \\
\text { BrW }\end{array}$ \\
\hline $\begin{array}{l}\text { ssw } 116 \\
\text { ssw } 117\end{array}$ & $\begin{array}{l}425829 \\
430018\end{array}$ & $\begin{array}{l}705328 \\
705430\end{array}$ & $\begin{array}{l}\text { FIBERGLASS PLUS } \\
\text { MAYIAN }\end{array}$ & $\begin{array}{l}1984 \\
1984\end{array}$ & $\begin{array}{r}80 \\
100\end{array}$ & -- & -- & -- & $\begin{array}{l}\mathbf{x} \\
\mathbf{x}\end{array}$ & -- & $\begin{array}{l}\text { Brw } \\
\text { BrW }\end{array}$ \\
\hline
\end{tabular}

STRAFFORD COUNTY

Barrington

\begin{tabular}{|c|c|c|c|c|c|c|c|c|c|c|c|c|}
\hline $\begin{array}{l}\text { BBW } \\
\text { BBW }\end{array}$ & $\begin{array}{l}48 \\
49\end{array}$ & $\begin{array}{l}431044 \\
431045\end{array}$ & $\begin{array}{l}710201 \\
710202\end{array}$ & $\begin{array}{l}\text { O H MATERIALS } \\
\text { NHWSPCC }\end{array}$ & $\begin{array}{l}1984 \\
1984\end{array}$ & $\begin{array}{l}335 \\
335\end{array}$ & $\begin{array}{l}2 \\
2\end{array}$ & $\begin{array}{l}6.8 \\
2.3\end{array}$ & $\begin{array}{l}\mathbf{P} \\
\mathbf{P}\end{array}$ & $\begin{array}{l}\mathbf{P} \\
\mathbf{P}\end{array}$ & $\begin{array}{l}16.8 \\
52.3\end{array}$ & $\begin{array}{l}\text { Bor } \\
\text { Bor }\end{array}$ \\
\hline \multicolumn{13}{|c|}{ Dover } \\
\hline $\begin{array}{l}\text { DJS } \\
\text { DJS }\end{array}$ & 1 & 430941 & 705307 & COLPRIT I $W$ & -- & 80 & -- & -- & -- & -- & -- & sp \\
\hline DJS & 2 & 430921 & 705247 & BURNELL MRS CHARLES $W$ & -- & 90 & -- & - & -- & -- & -- & Sp \\
\hline DJW & 8 & 431025 & 705406 & USCOE & 1952 & 97.4 & 3 & - & $\mathbf{s}$ & -- & -- & Wsh \\
\hline DJW & 11 & 431017 & 705420 & USCOE & 1952 & 98.6 & 3 & -- & $\mathbf{s}$ & -- & -- & Wsh \\
\hline DJW & 12 & 431021 & 705432 & USCOE & 1952 & 106.0 & 3 & -- & $\mathbf{s}$ & -- & -- & Wsh \\
\hline DJW & 13 & 431023 & 705435 & USCOE & 1952 & 100 & 3 & - & $\mathbf{s}$ & -- & -- & Wrsh \\
\hline DJW & 14 & 431025 & 705402 & USCOE & 1952 & 105.3 & 3 & - & $\mathbf{s}$ & -- & -- & Wsh \\
\hline DJW & 15 & 431027 & 705359 & USCOE & 1952 & 100 & 3 & -- & $\mathbf{s}$ & -- & -- & Wsh \\
\hline DJW & 46 & 431030 & 705429 & USCOE & 1952 & 90 & 3 & -- & $\mathbf{s}$ & $-\infty$ & -- & Wsh \\
\hline DJW & 47 & 431030 & 705429 & USCOE & 1952 & 90 & 3 & -- & $\mathbf{s}$ & -- & -- & Wsh \\
\hline DJW & 48 & 431031 & 705422 & USCOE & 1952 & 90 & 3 & -- & $\mathbf{s}$ & -- & -- & Wsh \\
\hline DJW & 49 & 431032 & 705416 & USCOE & 1952 & 90 & 3 & -- & $\mathbf{s}$ & -- & -- & Wsh \\
\hline DJW & 50 & 431019 & 705429 & USCOE & 1952 & 100 & 3 & -- & $\mathbf{s}$ & -- & -- & Vsh \\
\hline
\end{tabular}


and springs--Continued

\begin{tabular}{|c|c|c|c|c|c|c|}
\hline Water & C level & & $\begin{array}{l}\text { Maxi- } \\
\text { mum } \\
\text { well } \\
\text { yield }\end{array}$ & Draw- & $\begin{array}{l}\text { Specific } \\
\text { capa- } \\
\text { city } \\
\text { l(gal/ Pumping }\end{array}$ & \\
\hline $\begin{array}{l}\text { Depth } \\
\text { (ft) }\end{array}$ & $\begin{array}{c}\text { Date } \\
(\mathrm{mm}-\mathrm{dd}-\mathrm{yy})\end{array}$ & Use & $\begin{array}{l}\text { (gal/ } \\
\text { min) }\end{array}$ & $\begin{array}{l}\text { down } \\
(f t)\end{array}$ & $\begin{array}{ll}\min ) / & \text { period } \\
\mathrm{ft}) & (\mathrm{hr})\end{array}$ & Remarks \\
\hline
\end{tabular}

Stratham--Cont inued

\begin{tabular}{|c|c|c|c|c|c|}
\hline-- & -- & $\mathrm{H}$ & 20 & -- & -- \\
\hline-- & - & H & 10 & -- & -- \\
\hline-- & -- & $\mathrm{H}$ & -- & -- & -- \\
\hline- & -- & $\mathrm{H}$ & 10 & -- & -- \\
\hline - & -- & H & 30 & -- & -- \\
\hline- & -- & H & 20 & - & - \\
\hline- & - & $\mathrm{H}$ & 30 & -- & -- \\
\hline- & -- & $\mathrm{H}$ & 25 & -- & -- \\
\hline- & -- & $\mathrm{H}$ & 20 & -- & -- \\
\hline- & -- & $\mathrm{H}$ & 5 & -- & -- \\
\hline- & -- & $\mathrm{H}$ & 8 & -- & -- \\
\hline- & - & $\mathrm{H}$ & 5 & -- & -- \\
\hline- & -- & $\mathrm{H}$ & 20 & -- & -- \\
\hline- & -- & $\mathrm{H}$ & 15 & -- & -- \\
\hline- & -- & -- & 8 & -- & -- \\
\hline- & -- & H & 10 & -- & -- \\
\hline . & - & $\mathrm{T}$ & 10.5 & -- & -- \\
\hline- & -- & $\mathrm{H}$ & 30 & -- & -- \\
\hline & -- & c & 5 & -- & -- \\
\hline 10 & $05-15-84$ & $\mathrm{H}$ & 20 & -- & -- \\
\hline
\end{tabular}

STRAFFORD COUNTY

\section{Barrington}

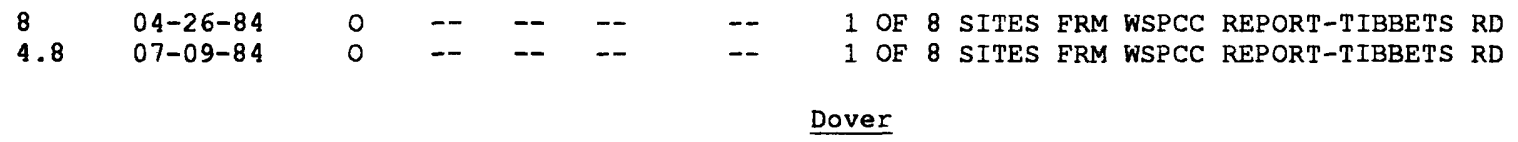

\begin{tabular}{|c|c|c|c|c|c|c|}
\hline-- & -- & $\mathrm{H}, \mathrm{S}$ & 3 & -- & - & -- \\
\hline-- & -- & $\mathrm{H}$ & -- & -- & & -- \\
\hline 9.7 & $11-24-53$ & 0 & -- & -- & & -- \\
\hline 9.5 & $01-18-54$ & 0 & -- & -- & - & - \\
\hline 9.8 & $01-21-54$ & 0 & - & -- & - & - \\
\hline 1.3 & $01-21-54$ & 0 & -- & $\ldots$ & & - \\
\hline 9.1 & $01-21-54$ & 0 & -- & -- & & $-\infty$ \\
\hline-1.4 & $01-21-54$ & 0 & -- & - & & - \\
\hline 17.6 & $01-22-54$ & 0 & -- & -- & & -- \\
\hline 12.7 & $06-01-54$ & 0 & -- & $\ldots$ & & - \\
\hline 21.8 & $04-09-54$ & $\mathrm{H}$ & -- & - & & -- \\
\hline 7 & $04-09-54$ & $\mathrm{U}$ & -- & $\cdots$ & & -0 \\
\hline 10.9 & $04-12-54$ & 0 & -- & -- & & - \\
\hline 23.3 & $04-15-54$ & U & -- & -- & & - \\
\hline-- & -- & 0 & -- & - & & -6 \\
\hline- & - & 0 & - & - & & \\
\hline- & -- & 0 & -- & $\cdots$ & & \\
\hline-- & -- & 0 & -- & - & & \\
\hline & -- & 0 & - & 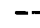 & & \\
\hline
\end{tabular}

CASING PLUGGED AT 17.5 FEET

USGS 


\begin{tabular}{|c|c|c|c|c|c|c|c|c|c|c|c|}
\hline & & & & & Altitude & Diam- & Depth to & & & $\begin{array}{l}\text { Depth to } \\
\text { bottom }\end{array}$ & \\
\hline $\begin{array}{l}\text { Local } \\
\text { site } \\
\text { number }\end{array}$ & $\begin{array}{l}\text { Lati- } \\
\text { tude }\end{array}$ & $\begin{array}{l}\text { Long- } \\
\text { tude }\end{array}$ & Owner or user & $\begin{array}{l}\text { Year } \\
\text { comp- } \\
\text { leted }\end{array}$ & $\begin{array}{c}\text { above } \\
\text { sea } \\
\text { level } \\
\text { (ft) }\end{array}$ & $\begin{array}{c}\text { eter } \\
\text { of } \\
\text { well } \\
\text { (in.) }\end{array}$ & $\begin{array}{l}\text { bottom } \\
\text { of } \\
\text { casing } \\
\text { (ft) }\end{array}$ & $\begin{array}{c}\text { Casing } \\
\text { mate- } \\
\text { rial }\end{array}$ & $\begin{array}{l}\text { Type } \\
\text { of } \\
\text { fin- } \\
\text { ish }\end{array}$ & $\begin{array}{l}\text { of } \\
\text { open } \\
\text { section } \\
\text { (ft) }\end{array}$ & $\begin{array}{l}\text { Type } \\
\text { of } \\
\text { site }\end{array}$ \\
\hline
\end{tabular}

Dover--Cont inued

$\begin{array}{llll}\text { DJW } & 51 & 431017 & 705420 \\ \text { DJW } & 52 & 431020 & 705416 \\ \text { DJW } & 53 & 431024 & 705411 \\ \text { DJW } & 54 & 431024 & 705411 \\ \text { DJW } & 55 & 431028 & 705353 \\ & & & \\ \text { DJW } & 56 & 431030 & 705349 \\ \text { DJW } & 57 & 431033 & 705340 \\ \text { DJW } & 58 & 431030 & 705330 \\ \text { DJW } & 59 & 431023 & 705401 \\ \text { DJW } & 60 & 431024 & 705300 \\ & & & \\ \text { DJW } & 61 & 430949 & 705346 \\ \text { DJW } & 62 & 430943 & 705317 \\ \text { DJW } & 63 & 430936 & 705332 \\ \text { DJW } & 69 & 430943 & 705233 \\ \text { DJW } & 73 & 430918 & 705241 \\ & & & \\ \text { DJW } & 74 & 430955 & 705336 \\ \text { DJW } & 77 & 430957 & 705225 \\ \text { DJW } & 84 & 431002 & 705408 \\ \text { DJW } & 88 & 431025 & 705404 \\ \text { DJW } & 89 & 431008 & 705421 \\ & & & \end{array}$

$\begin{array}{lcc}\text { USCOE } & 1952 & 98.6 \\ \text { USCOE } & 1952 & 103.1 \\ \text { USCOE } & 1952 & 100 \\ \text { USCOE } & 1952 & 97.1 \\ \text { USCOE } & 1952 & 90 \\ & & \\ \text { USCOE } & 1952 & 90 \\ \text { USCOE } & 1952 & 90 \\ \text { USCOE } & 1952 & 110 \\ \text { USCOE } & 1952 & 130 \\ \text { USCOE } & 1952 & 100 \\ & & \\ \text { USCOE } & 1952 & 80 \\ \text { USCOE } & 1952 & 60 \\ \text { USCOE } & 1952 & 70 \\ \text { NEWSKY JOSEPH } & 1936 & 130 \\ \text { JENSVILL FRED } & 1934 & 110 \\ & & \\ \text { DOVER, CITY OF } & 1950 & 100 \\ \text { DOVER, CITY OF } & 1950 & 150 \\ \text { DOVER SAND \& GRAVEL } & 1957 & 160 \\ \text { DOVER, CITY OF } & -- & 80 \\ \text { DOVER, CITY OF } & -- & 130 \\ \end{array}$

$\begin{array}{rr}8 & -- \\ 8 & -- \\ 8 & -- \\ 8 & -- \\ 8 & -- \\ 3 & -- \\ 3 & -- \\ 3 & -- \\ 8 & -- \\ 3 & -- \\ 3 & -- \\ 3 & -- \\ 3 & -- \\ -- & -- \\ -- & -- \\ 2 & -- \\ 8 & -- \\ 8 & -- \\ -- & -- \\ -- & --\end{array}$

$\begin{array}{cccc}\text { s } & -- & -- & \text { Cbl } \\ \text { S } & -- & -- & \text { Cbl } \\ \text { s } & -- & -- & \text { BrW } \\ \text { s } & -- & -- & \text { Cbl } \\ \text { s } & -- & -- & \text { BrW } \\ \text { s } & -- & -- & \text { Wsh } \\ \text { S } & -- & -- & \text { Wsh } \\ \text { S } & -- & -- & \text { Wsh } \\ \text { s } & -- & -- & \text { Cbl } \\ \text { S } & -- & -- & \text { Wsh } \\ & & & \\ \text { S } & -- & -- & \text { Wsh } \\ \text { s } & -- & -- & \text { Wsh } \\ \text { S } & -- & -- & \text { Wsh } \\ -- & -- & -- & \text { BrW } \\ -- & -- & -- & \text { BrW } \\ \text { s } & -- & -- & \text { Wsh } \\ \text { s } & -- & -- & \text { BrW } \\ \text { s } & -- & -- & \text { Cbl } \\ -- & -- & -- & -- \\ -- & -- & -- & --\end{array}$

Durham

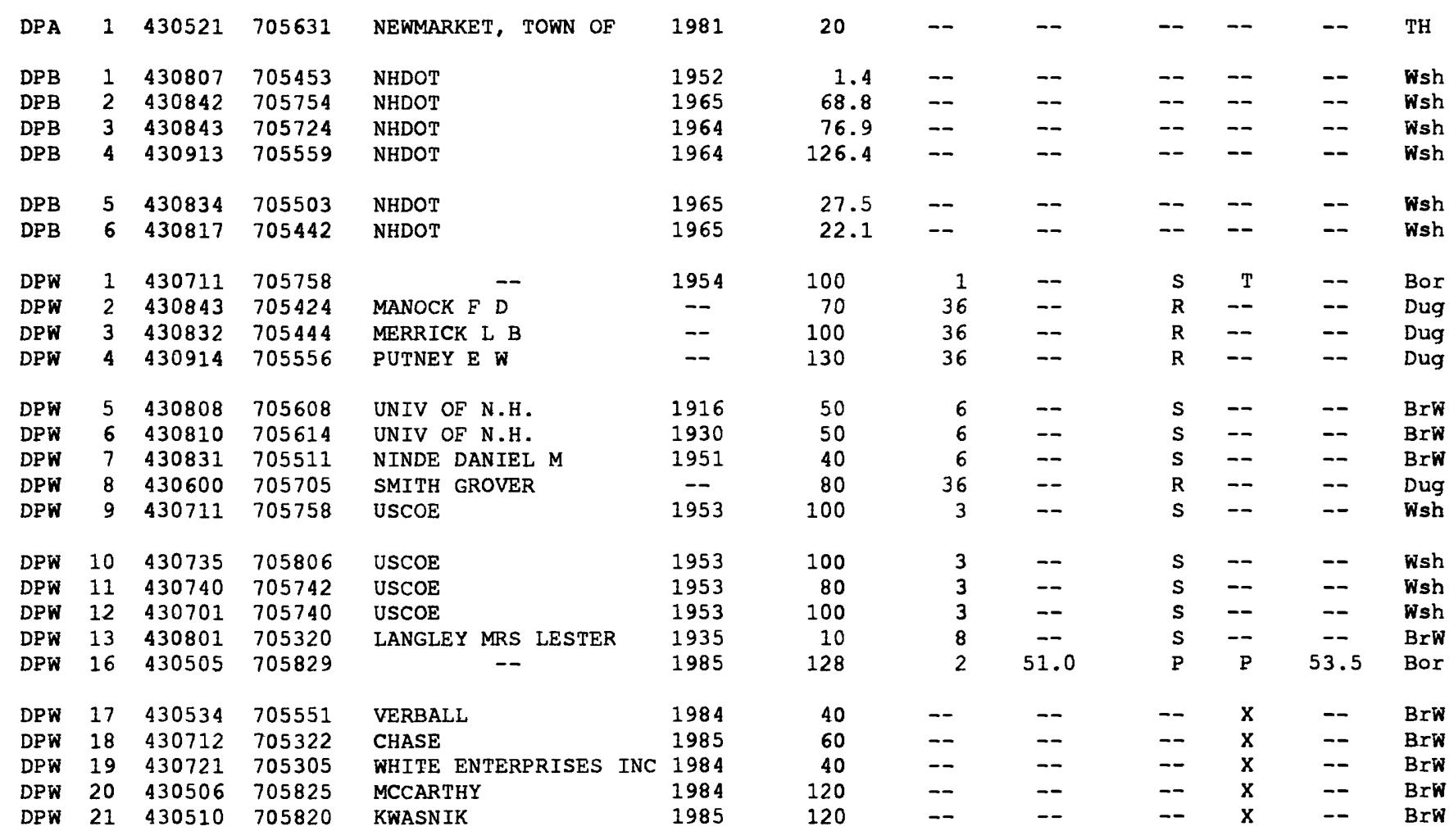


and springs--Continued

\begin{tabular}{|c|c|c|c|c|c|c|}
\hline Water & level & & $\begin{array}{l}\text { Maxi- } \\
\text { mum } \\
\text { well } \\
\text { yield }\end{array}$ & Draw- & $\begin{array}{l}\text { Specific } \\
\text { capa- } \\
\text { city } \\
\text { (lgal/ Pumping }\end{array}$ & \\
\hline
\end{tabular}

Dover--Continued

\begin{tabular}{|c|c|c|c|c|c|}
\hline- & -- & 0 & -- & -- & -- \\
\hline - & - & 0 & -- & - & -- \\
\hline -- & -- & 0 & -- & -- & -- \\
\hline-- & -- & 0 & -- & -- & -- \\
\hline-- & -- & 0 & -- & - & - \\
\hline- & -- & 0 & - & -- & -- \\
\hline-- & -- & 0 & -- & -- & -- \\
\hline-- & -- & 0 & -- & -- & -- \\
\hline 42 & $01-01-52$ & 0 & -- & - & -- \\
\hline-- & -- & 0 & - & -- & -- \\
\hline -- & $\ldots$ & 0 & -- & -. & -- \\
\hline-- & -- & 0 & -- & - & -- \\
\hline-- & -- & 0 & - & - & - \\
\hline- & -- & $\mathrm{H}$ & 3.5 & - & -- \\
\hline$m$ & -- & $\mathrm{H}$ & 3 & -- & -- \\
\hline-- & -- & 0 & -- & -- & - \\
\hline-- & -- & 0 & - & - & -- \\
\hline 56.8 & $04-30-57$ & 0 & -- & - & - \\
\hline-- & -- & $\mathrm{P}$ & -- & -- & - \\
\hline - & $\ldots$ & $P$ & -- & -- & -- \\
\hline
\end{tabular}

Durham

\begin{tabular}{|c|c|c|c|c|c|}
\hline-- & - & $\mathrm{T}$ & -- & - & -- \\
\hline- & -- & $\mathrm{T}$ & - & - & -- \\
\hline- & -- & $\mathrm{T}$ & -- & - & -- \\
\hline- & -- & $\mathrm{T}$ & -- & $\cdots$ & -- \\
\hline- & -- & $\mathrm{T}$ & - & -- & - \\
\hline - & -- & $\mathrm{T}$ & -. & $\ldots$ & -- \\
\hline & $\ldots$ & $\mathrm{T}$ & -. & - & $\ldots$ \\
\hline
\end{tabular}

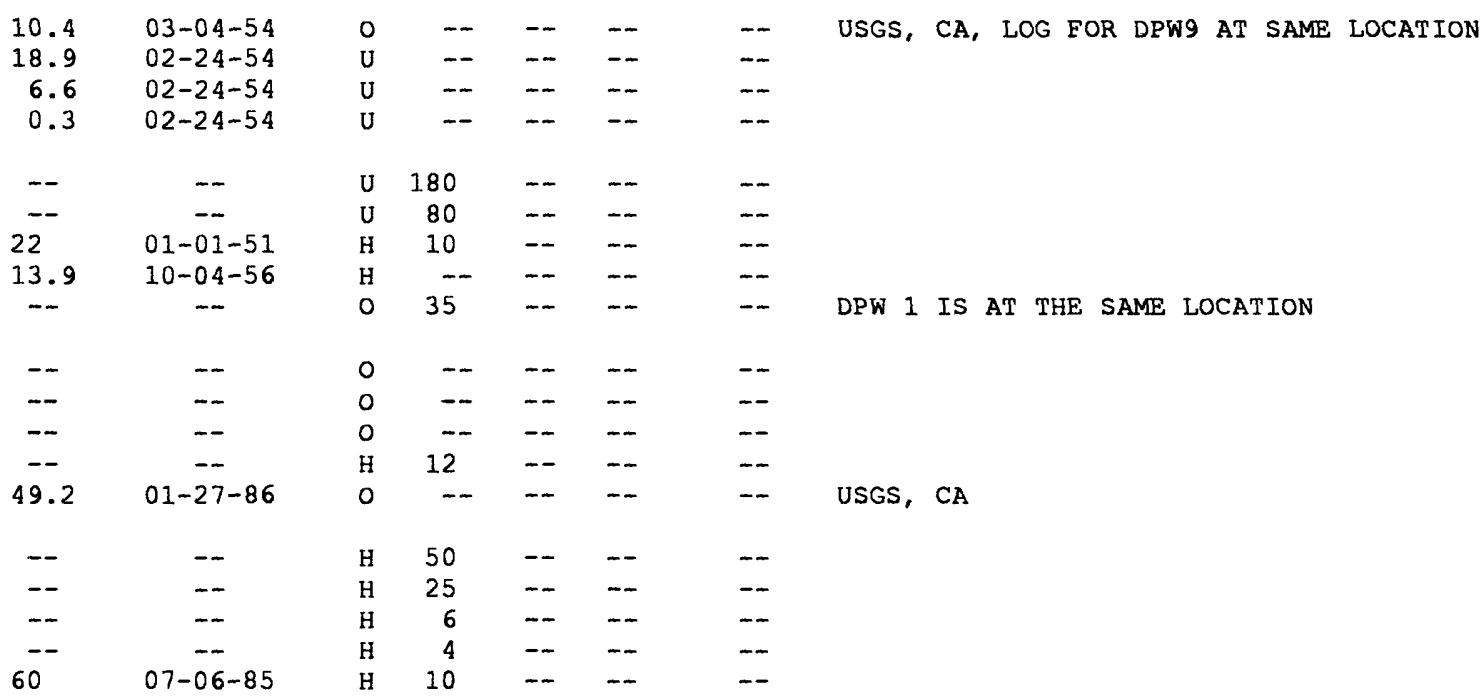


Table 2.--Description of wells, borings,

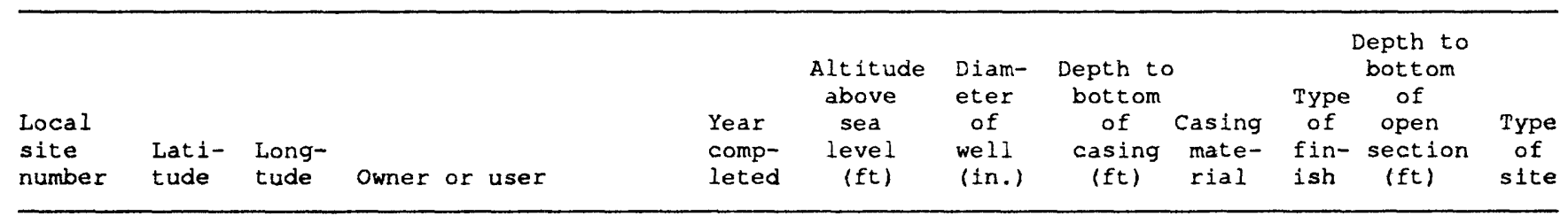

Durham--Cont inued

\begin{tabular}{|c|c|c|c|c|c|c|c|c|c|c|c|c|}
\hline DPW & 22 & 430515 & 705824 & BARVENIK & 1985 & 120 & -- & -- & -- & $\mathrm{x}$ & -- & $\mathrm{B} \perp \mathrm{W}$ \\
\hline DPW & 23 & 430527 & 705818 & GUARD & 1985 & 80 & -- & -- & -- & $\mathrm{x}$ & -- & $B r W$ \\
\hline DPW & 24 & 430554 & 705241 & CONGDON & 1984 & 60 & -- & -- & -- & $\mathrm{X}$ & -- & BrW \\
\hline DPW & 25 & 430805 & 705333 & SLUTZKY & 1984 & 20 & -- & -- & -- & $\mathrm{x}$ & -- & $\mathrm{BrW}$ \\
\hline DPW & 26 & 430734 & 705518 & CHAMBERLAIN & 1984 & 60 & -- & -- & -- & $\mathrm{x}$ & -- & $\mathrm{B} \times \mathrm{W}$ \\
\hline DPW & 27 & 430734 & 705329 & JENKINS & 1984 & 20 & -- & -- & -- & $\mathrm{x}$ & -- & $\mathrm{B} r \mathrm{~W}$ \\
\hline DPW & 28 & 430614 & 705800 & CANFIEID & 1984 & 80 & -- & -- & - & $\mathrm{x}$ & -- & $B r W$ \\
\hline DPW & 29 & 430649 & 705209 & NELSON & 1985 & 20 & -- & -- & -- & $x$ & -- & BrW \\
\hline
\end{tabular}

Lee

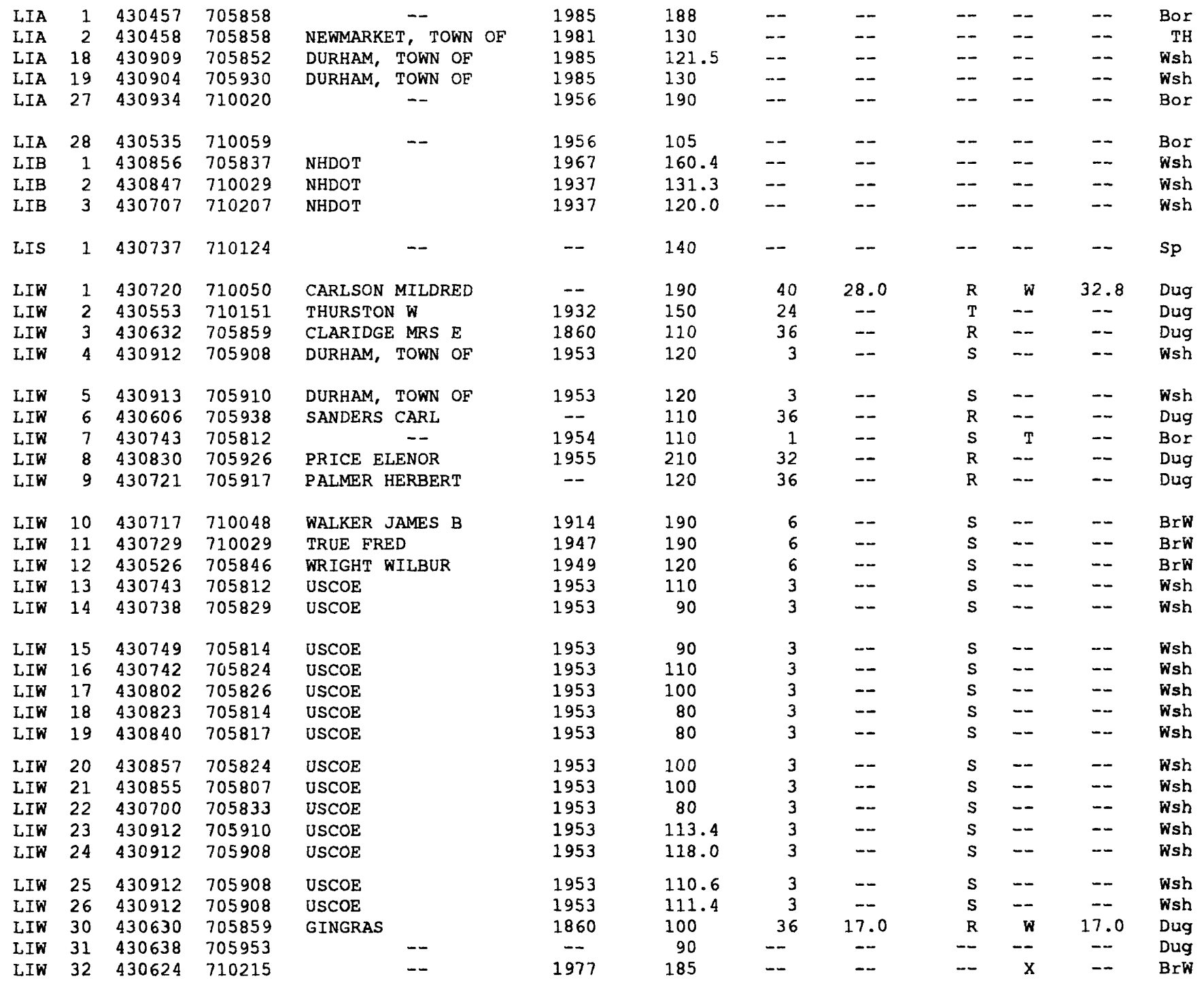


and springs--Continued

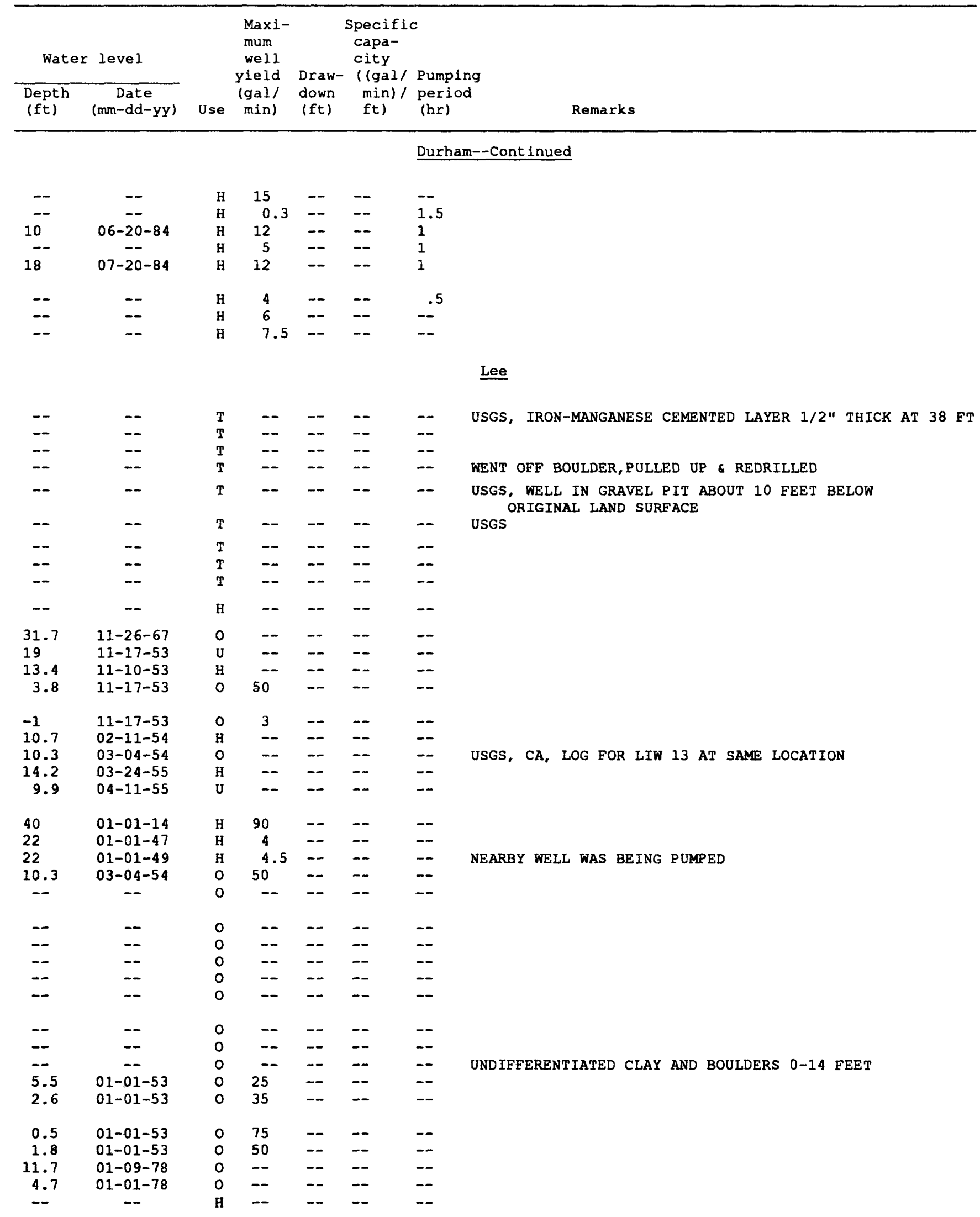




\begin{tabular}{|c|c|c|c|c|c|c|c|c|c|c|c|}
\hline $\begin{array}{l}\text { Local } \\
\text { site } \\
\text { number }\end{array}$ & $\begin{array}{l}\text { Lat1- } \\
\text { tude }\end{array}$ & $\begin{array}{l}\text { Long- } \\
\text { tude }\end{array}$ & Owner or user & $\begin{array}{l}\text { Year } \\
\text { comp- } \\
\text { leted }\end{array}$ & $\begin{array}{l}\text { Altitude } \\
\text { above } \\
\text { sea } \\
\text { level } \\
\text { (ft) }\end{array}$ & $\begin{array}{l}\text { Diam- } \\
\text { eter } \\
\text { of } \\
\text { well } \\
\text { (in.) }\end{array}$ & $\begin{array}{l}\text { Depth to } \\
\text { bottom } \\
\text { of } \\
\text { casing } \\
\text { (ft) }\end{array}$ & $\begin{array}{l}\text { Casing } \\
\text { mate- } \\
\text { rial }\end{array}$ & $\begin{array}{l}\text { Type } \\
\text { of } \\
\text { fin- } \\
\text { ish }\end{array}$ & $\begin{array}{l}\text { Depth to } \\
\text { bottom } \\
\text { of } \\
\text { open } \\
\text { section } \\
\text { (ft) }\end{array}$ & $\begin{array}{l}\text { Type } \\
\text { of } \\
\text { site }\end{array}$ \\
\hline
\end{tabular}

Lee--Cont inued

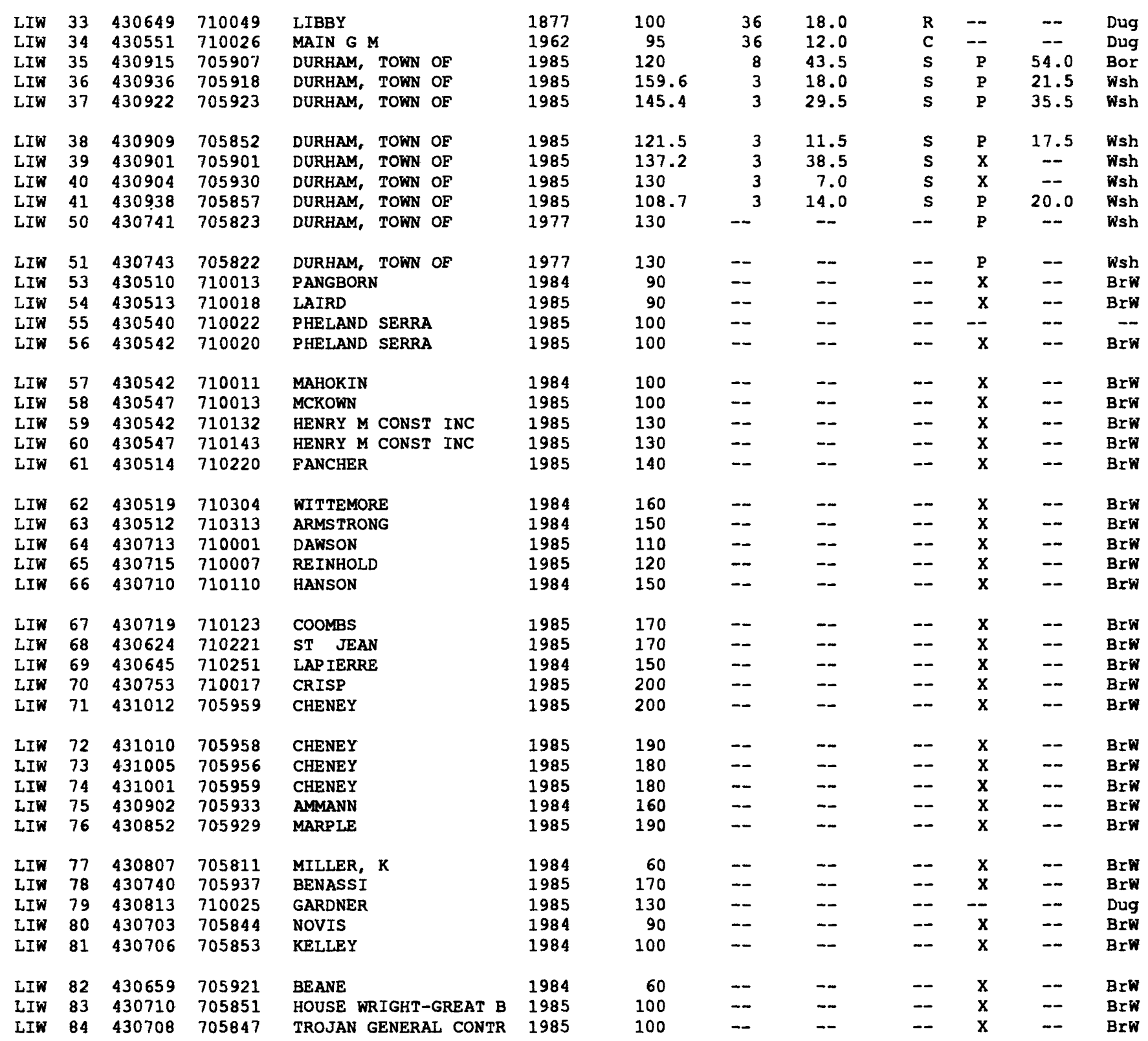

Madbury

$\begin{array}{llll}\text { MAA } & 1 & 431004 & 705508 \\ \text { MAA } & 2 & 431006 & 705507 \\ \text { MAA } & 3 & 431007 & 705510 \\ \text { MAA } & 4 & 431005 & 705505 \\ \text { MAA } & 5 & 431006 & 705507\end{array}$

MADBURY, TOWN OF MADBURY, TOWN OF MADBURY, TOWN OF MADBURY, TOWN OF MADBURY, TOWN OF

$\begin{array}{ll}1980 & 130 \\ 1980 & 150 \\ 1980 & 160 \\ 1980 & 150 \\ 1982 & 150\end{array}$

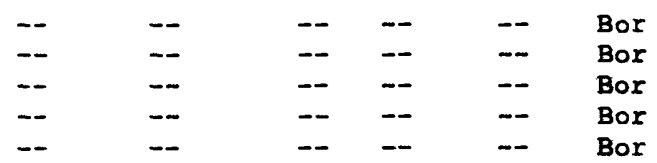




\begin{tabular}{|c|c|c|c|c|}
\hline Water level & \multirow{2}{*}{$\begin{array}{c}\text { Maxi- } \\
\text { mum } \\
\text { well } \\
\text { yield } \\
\text { (gal/ } \\
\text { Use min) }\end{array}$} & \multirow{2}{*}{\multicolumn{2}{|c|}{ 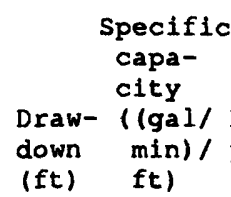 }} & \multirow[b]{2}{*}{ Remarks } \\
\hline $\begin{array}{c}\text { Date } \\
\text { (mm-dd-yy) }\end{array}$ & & & & \\
\hline
\end{tabular}

Lee--Continued

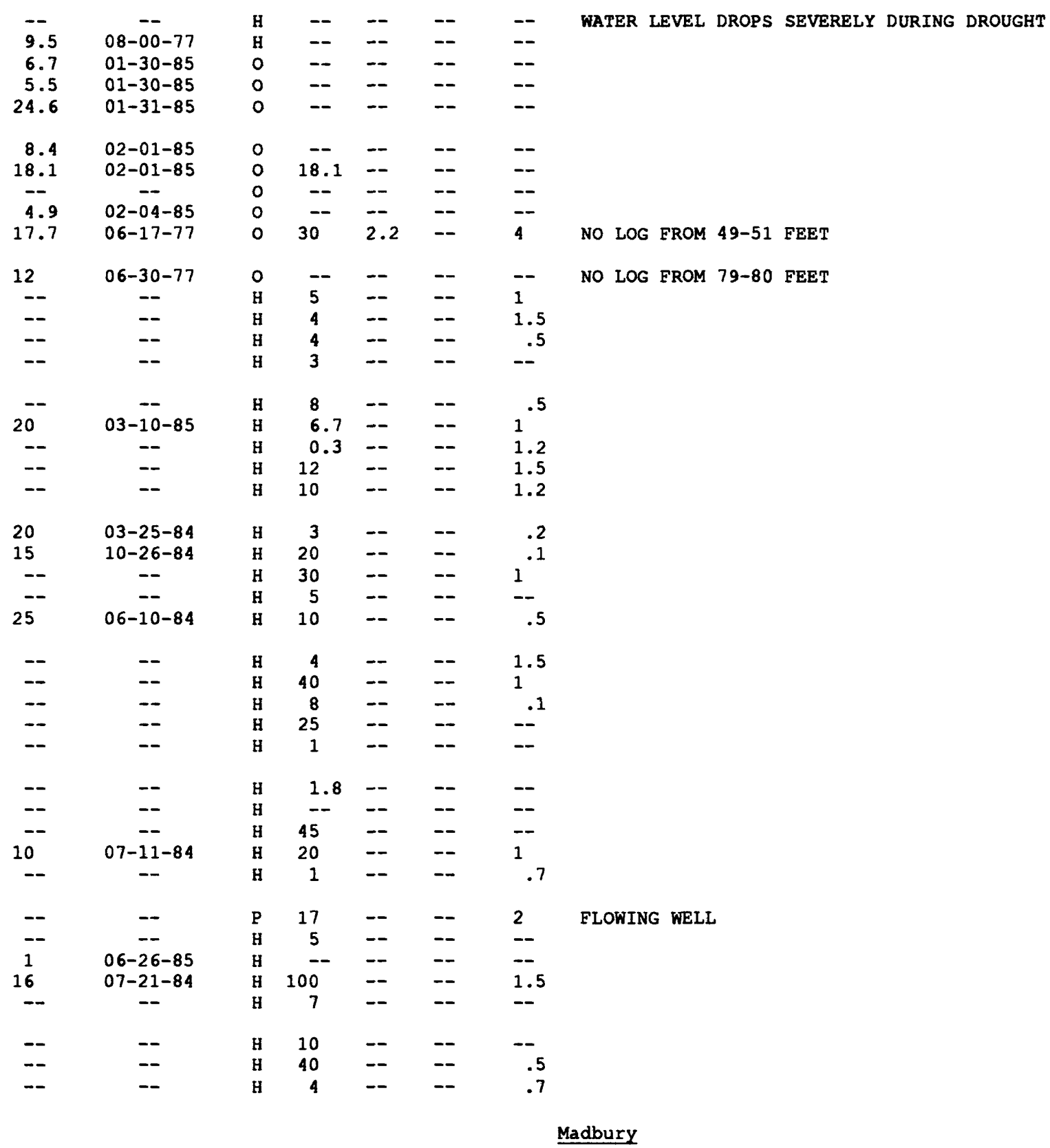

$\begin{array}{ccccccc}11 & 02-00-80 & T & -- & -- & -- & -- \\ 26 & 02-00-80 & T & -- & -- & -- & -- \\ -- & -- & T & -- & -- & -- & -- \\ -- & -- & T & -- & -- & -- & -- \\ -- & -- & T & -- & -- & -- & --\end{array}$


Table 2.--Description of wells, borings,

\begin{tabular}{|c|c|c|c|c|c|c|c|c|c|c|c|}
\hline $\begin{array}{l}\text { Loca } 1 \\
\text { site } \\
\text { number }\end{array}$ & $\begin{array}{l}\text { Lat } 1- \\
\text { tude }\end{array}$ & $\begin{array}{l}\text { Long- } \\
\text { tude }\end{array}$ & Owner or user & $\begin{array}{l}\text { Year } \\
\text { comp- } \\
\text { leted }\end{array}$ & $\begin{array}{l}\text { Altitude } \\
\text { above } \\
\text { sea } \\
\text { level } \\
\text { (ft) }\end{array}$ & $\begin{array}{l}\text { Diam- } \\
\text { eter } \\
\text { of } \\
\text { well } \\
\text { (in.) }\end{array}$ & $\begin{array}{c}\text { Depth to } \\
\text { bottom } \\
\text { of } \\
\text { casing } \\
\text { (ft) }\end{array}$ & $\begin{array}{l}\text { Casing } \\
\text { mate- } \\
\text { rial }\end{array}$ & $\begin{array}{l}\text { Type } \\
\text { of } \\
\text { fin- } \\
\text { ish }\end{array}$ & $\begin{array}{l}\text { Depth to } \\
\text { bottom } \\
\text { of } \\
\text { open } \\
\text { section } \\
\text { (ft) }\end{array}$ & $\begin{array}{c}\text { Type } \\
\text { of } \\
\text { site }\end{array}$ \\
\hline
\end{tabular}

Madbury--Cont inued

\begin{tabular}{|c|c|c|c|c|c|c|c|c|c|c|c|c|}
\hline MAW & 1 & 430858 & 705325 & USCOE & 1952 & 30.7 & 3 & -- & $\mathbf{S}$ & -- & -- & Wsh \\
\hline MAW & 2 & 430901 & 705319 & USCOE & 1952 & 33.0 & 3 & -- & $\mathbf{s}$ & -- & -- & Wsh \\
\hline MAT & 3 & 430903 & 705321 & USCOE & 1952 & 31.3 & 3 & -- & $\mathbf{s}$ & - & -- & Wsh \\
\hline MAW & 4 & 430906 & 705319 & USCOE & 1952 & 32.7 & 3 & -- & $\mathbf{S}$ & -- & -- & Wsh \\
\hline MAW & 5 & 430913 & 705319 & USCOE & 1952 & 47.3 & 3 & -- & $\mathbf{s}$ & - & $-\infty$ & Wsh \\
\hline MAN & 6 & 430920 & 205323 & USCOE & 1952 & 39.1 & 3 & -- & $\mathbf{S}$ & -- & -- & Msh \\
\hline MAW & 8 & 430933 & 705346 & CAMPBELL E P & 1932 & 110 & 6 & -- & $\mathbf{s}$ & -- & -- & Brw \\
\hline MAN & 11 & 430853 & 705325 & PORTSMOUTH, CITY OF & 1954 & 31.1 & 24 & -- & $\mathbf{S}$ & - & -- & $\mathrm{Cbl}$ \\
\hline MAW & 12 & 430901 & 705319 & PORTSMOUTH, CITY OF & 1954 & 32.7 & 24 & -- & $\mathbf{s}$ & -- & -- & $\mathrm{Cbl}$ \\
\hline MAT & 13 & 430906 & 705319 & PORTSMOUTH, CITY OF & 1954 & 32.7 & 24 & -- & $\mathbf{s}$ & -- & -- & $\mathrm{Cbl}$ \\
\hline MAW & 14 & 430920 & 705323 & PORTSMOUTH, CITY OF & 1954 & 39.0 & 24 & -- & $\mathbf{s}$ & -- & -- & $\mathrm{Cbl}$ \\
\hline MAW & 21 & 431023 & 705449 & USCOE & 1952 & 90 & 3 & -- & $\mathbf{S}$ & -- & -- & Wsh \\
\hline MAW & 22 & 431019 & 705448 & USCOE & 1952 & 100 & 3 & -- & $\mathbf{S}$ & -- & -- & Wsh \\
\hline MAW & 23 & 431017 & 705439 & USCOE & 1952 & 100 & 3 & -- & $s$ & -- & -- & Wsh \\
\hline MAT & 24 & 431013 & 705453 & USCOE & 1952 & 100 & 3 & -- & $\mathbf{S}$ & -- & -- & Wsh \\
\hline MAN & 25 & 431014 & 705448 & USCOE & 1952 & 100 & 3 & -- & $\mathbf{S}$ & -- & - & Hsh \\
\hline MAE & 26 & 431016 & 705433 & USCOE & 1952 & 100 & 3 & -- & $\mathbf{S}$ & -- & -- & Wsh \\
\hline MAW & 27 & 431008 & 705422 & USCOE & 1952 & 150 & 3 & -- & $\mathbf{S}$ & -- & -- & Wsh \\
\hline MAW & 28 & 431004 & 705427 & USCOE & 1952 & 150 & 3 & -- & $\mathbf{S}$ & -- & -- & Hsh \\
\hline MAT & 29 & 430952 & 705405 & USCOE & 1952 & 120 & 3 & $-\infty$ & $\mathbf{S}$ & -- & -- & Wsh \\
\hline MAN & 30 & 430951 & 705359 & USCOE & 1952 & 110 & 3 & -- & $\mathbf{S}$ & -- & -- & Hsh \\
\hline MAH & 31 & 430948 & 705402 & USCOE & 1952 & 90 & 3 & -- & $\mathbf{S}$ & -- & -- & Wsh \\
\hline MAH & 32 & 430944 & 705406 & USCOE & 1952 & 80 & 3 & -- & $\mathbf{S}$ & -- & -- & Wsh \\
\hline MAH & 33 & 430940 & 705411 & USCOE & 1952 & 80 & 3 & -- & $\mathbf{S}$ & -- & -- & Msh \\
\hline MAW & 36 & 430944 & 705417 & USCOE & 1952 & 90 & 3 & -- & $\mathbf{S}$ & -- & -- & Hsh \\
\hline MAK & 37 & 430941 & 705421 & USCOE & 1952 & 80 & 3 & -- & $\mathbf{S}$ & -- & -- & Tsh \\
\hline MAH & 41 & 430.938 & 705427 & USCOE & 1952 & 70 & 3 & -- & $\mathbf{S}$ & -- & -- & Msh \\
\hline MAN & 42 & 430941 & 705433 & USCOE & 1952 & 60 & 3 & -- & $\mathbf{S}$ & -- & -- & Wsh \\
\hline MAW & 43 & 430936 & 705427 & USCOE & 1952 & 50 & 3 & -- & $\mathbf{S}$ & -- & -- & Hsh \\
\hline MAW & 44 & 430930 & 705420 & USCOE & 1952 & 40 & 3 & -- & $\mathbf{S}$ & -- & -- & Wsh \\
\hline MAN & 45 & 430954 & 705447 & USCOE & 1952 & 110 & 3 & -- & $\mathbf{S}$ & -- & -- & Wsh \\
\hline MAW & 46 & 430946 & 705450 & USCOE & 1952 & 120 & 3 & -- & $\mathbf{s}$ & -- & -- & Wsh \\
\hline MAN & 47 & 430909 & 705318 & USCOE & 1952 & 30 & 3 & -- & $\mathbf{S}$ & -- & -- & Wsh \\
\hline MAN & 48 & 430904 & 705319 & USCOE & 1952 & 30 & 3 & $-\infty$ & $\mathbf{S}$ & -- & $-\infty$ & Wsh \\
\hline MAN & 49 & 430903 & 705319 & USCOE & 1952 & 30 & 3 & -- & $\mathbf{s}$ & -- & -- & Wsh \\
\hline MAN & 50 & 430900 & 705331 & USCOE & 1952 & 30 & 3 & -- & $\mathbf{s}$ & -- & -- & Wsh \\
\hline MAT & 51 & 430861 & 705338 & USCOE & 1952 & 40 & 3 & -- & $s$ & -- & - & Wsh \\
\hline MAW & 52 & 430902 & 705342 & USCOE & 1952 & 30 & 3 & -- & $\mathbf{s}$ & -- & -- & Wsh \\
\hline MAW & 56 & 430954 & 705405 & -- & 1985 & 128 & 2 & 67.0 & $\mathbf{P}$ & $\mathbf{P}$ & 69.5 & Bor \\
\hline MAN & 57 & 431003 & 705455 & -- & 1985 & 156 & 2 & 69.5 & $\mathbf{P}$ & $\mathbf{P}$ & 72.1 & Bor \\
\hline MAN & 58 & 430935 & 705352 & MADBURY METALS & 1985 & 102.5 & -- & -- & -- & -- & -- & - \\
\hline MAT & 59 & 431011 & 705448 & MADBURY METALS & 1985 & 113.7 & -- & -- & -- & -- & -- & - \\
\hline MAN & 60 & 431008 & 705440 & MADBURY METALS & 1985 & 125.4 & -- & -- & -- & -- & -- & - \\
\hline MAK & 61 & 431004 & 705440 & MADBURY METALS & 1985 & 109.1 & -- & -- & -- & -- & -- & BrW \\
\hline MAN & 62 & 431009 & 705456 & MADBURY METALS & 1985 & 99.0 & -- & $-\infty$ & -- & -- & -- & \\
\hline MAN & 63 & 431008 & 705502 & MADBURY METALS & 1985 & 97.7 & $-\infty$ & -- & -- & -- & -- & -- \\
\hline MAN & 65 & 431002 & 705508 & MADBURY, TOWN OF & 1982 & 120 & $-\infty$ & -- & -- & -- & -- & Bor \\
\hline MAN & 66 & 431003 & 705510 & MADBURY, TOWN OE & 1982 & 120 & -- & -- & -- & -- & -- & Bor \\
\hline
\end{tabular}




\begin{tabular}{|c|c|c|c|c|c|c|c|}
\hline \multicolumn{2}{|c|}{ Water level } & Use & $\begin{array}{l}\text { Maxi- } \\
\text { mum } \\
\text { well } \\
\text { yield } \\
\text { (gal/ } \\
\text { min) }\end{array}$ & $\begin{array}{l} \\
\text { Draw- } \\
\text { down } \\
\text { (ft) }\end{array}$ & $\begin{array}{l}\text { Specific } \\
\text { capa- } \\
\text { city } \\
\text { ((gal/ } \\
\text { min)/ } \\
\text { ft) }\end{array}$ & $\begin{array}{l}\text { c } \\
\text { Pumping } \\
\text { period } \\
\text { (hr) }\end{array}$ & Remarks \\
\hline \multicolumn{8}{|c|}{ Madbury--Continued } \\
\hline $\begin{array}{l}3.3 \\
6.3 \\
3.6 \\
0.9\end{array}$ & $\begin{array}{l}12-17-53 \\
01-13-54 \\
01-21-54 \\
01-21-54\end{array}$ & $\begin{array}{l}0 \\
0 \\
0 \\
0\end{array}$ & $\begin{array}{l}-- \\
=- \\
--\end{array}$ & $\begin{array}{l}-- \\
-- \\
--\end{array}$ & $\begin{array}{l}-- \\
-- \\
--\end{array}$ & $\begin{array}{l}-- \\
-- \\
--\end{array}$ & $\begin{array}{l}\text { WELL DESTROYED } \\
\text { WELL DESTROYED }\end{array}$ \\
\hline $\begin{array}{c}13.6 \\
4.4 \\
32 \\
4 \\
4.9\end{array}$ & $\begin{array}{l}01-21-54 \\
01-26-54 \\
01-01-32 \\
01-01-54 \\
01-01-54\end{array}$ & $\begin{array}{l}O \\
O \\
H \\
P \\
P\end{array}$ & $\begin{array}{l}-- \\
-- \\
1.5 \\
-- \\
--\end{array}$ & $\begin{array}{l}= \\
-- \\
-- \\
--\end{array}$ & $\begin{array}{l}-- \\
=- \\
-- \\
--\end{array}$ & $\begin{array}{l}-- \\
=- \\
-- \\
--\end{array}$ & $\begin{array}{l}\text { WELL DESTROYED } \\
\text { WELL DESTROYED }\end{array}$ \\
\hline $\begin{array}{l}0.8 \\
3.4 \\
-- \\
--\end{array}$ & $\begin{array}{c}01-01-54 \\
03-21-55 \\
-- \\
-- \\
--\end{array}$ & $\begin{array}{l}P \\
P \\
0 \\
0 \\
0\end{array}$ & $\begin{array}{c}900 \\
-- \\
-- \\
--\end{array}$ & $\begin{array}{l}-- \\
-- \\
--\end{array}$ & $\begin{array}{l}-- \\
-- \\
-- \\
--\end{array}$ & $\begin{array}{l}-- \\
-- \\
-- \\
--\end{array}$ & \\
\hline $\begin{array}{l}-- \\
\overline{8.5} \\
27\end{array}$ & $\begin{array}{c}\overline{--} \\
-\overline{01-01-52} \\
01-01-52\end{array}$ & $\begin{array}{l}0 \\
0 \\
0 \\
0\end{array}$ & $\begin{array}{l}-- \\
-- \\
--\end{array}$ & $\begin{array}{l}-- \\
-- \\
-\end{array}$ & $\begin{array}{l}-- \\
-- \\
--\end{array}$ & $\begin{array}{l}-- \\
-- \\
--\end{array}$ & \\
\hline-- & -- & 0 & -- & -- & -- & -- & \\
\hline$=$ & $=$ & $\begin{array}{l}0 \\
0\end{array}$ & $\overline{-}$ & $=$ & $=$ & $=$ & \\
\hline-- & -- & 0 & -- & - & -- & -- & \\
\hline -- & -- & $\begin{array}{l}0 \\
0\end{array}$ & $=$ & 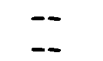 & $=$ & $=$ & \\
\hline-- & -- & 0 & -- & -- & -- & -- & \\
\hline-- & -- & 0 & -- & -- & -- & -- & \\
\hline$\overline{--}$ & $=$ & $\begin{array}{l}0 \\
0\end{array}$ & $=$ & $=$ & $=$ & -- & \\
\hline-- & -- & 0 & -- & -- & -- & -- & \\
\hline-- & -- & 0 & -- & - & -- & -- & \\
\hline-- & -- & 0 & -- & -- & -- & -- & \\
\hline $\begin{array}{l}-- \\
-\end{array}$ & $=$ & $\begin{array}{l}0 \\
0\end{array}$ & $=$ & $\overline{-}$ & $\begin{array}{l}-- \\
--\end{array}$ & $\overline{-}$ & \\
\hline-- & -- & 0 & -- & -- & -- & -- & \\
\hline -- & -- & 0 & -- & -- & -- & -- & \\
\hline-- & -- & 0 & -- & - & -- & -- & \\
\hline-- & -- & 0 & -- & -- & -- & -- & \\
\hline 39 & $11-26-85$ & 0 & - & - & - & - & USGS \\
\hline 67.8 & $11-26-85$ & 0 & - & -- & - & -- & USGS, CA \\
\hline 13 & $07-25-85$ & 0 & -- & -- & -- & -- & \\
\hline 24.9 & $07-25-85$ & 0 & -- & -- & -- & -- & \\
\hline 37.9 & $07-25-85$ & 0 & -- & -- & -- & -- & \\
\hline 21.9 & $07-25-85$ & 0 & -- & -- & -- & -- & \\
\hline 9.7 & $07-25-85$ & 0 & -- & -- & -- & -- & \\
\hline 7.3 & $07-25-85$ & 0 & -- & -- & -- & -- & \\
\hline 25 & $03-00-82$ & 0 & -- & -- & -- & -- & \\
\hline 4 & $03-00-82$ & 0 & -- & -- & -- & -- & \\
\hline
\end{tabular}





\section{Table 3.--Lithologic logs of wells and borings}

Local site number: First two characters indicate U.S. Geological Survey town code. Third character indicates-A, borings done for hydrologic purposes; B, borings done primarily for constructional purposes.

Depth drilled: Depth drilled in feet below land-surface datum.

Depth of well: Depth of well in feet below land-surface datum.

Depth to refusal: Depth to bedrock or refusal in feet below land-surface datum.

Depth to top: Depth to top of each lithologic unit in feet below land-surface datum.

\section{Aquifer code:}

Codes for the following aquifer materials and geologic ages are given as follows:

110MRER Marine and estuarine deposits, Quaternary

10SDMN Sediments, undifferentiated, Quaternary

110SOIL Soil, Quaternary

111FILL Fill, Holocene

111SDMN Sediments, undifferentiated, Holocene

111SWMP Swamp deposits, Holocene

111SOIL Soil, Holocene

112DELT Delta deposits, Pleistocene
$112 \mathrm{GLCL}$ Glacial deposit, undifferentiated, Pleistocene

$112 \mid$ CCC lco-contact deposits, Pleistocene

112MRIN Marine deposits (may include estuarine deposits)

12NRSR Nearshore deposits (marine and non-marine), Pleistocene

1120TSH Outwash, Pleistocene

112SRFD Stratified deposits, undifferentiated, Pleistocene

112TILL Till, Pleistocene bedrock, bedrock undifferentiated

\section{Lithology:}

The following lithologic codes are used to describe aquifer units:

\begin{tabular}{|c|c|c|c|c|c|}
\hline BLDR & Boulders & GRSC & Gravel, silt and clay & SDGL & Sand and gravel \\
\hline BRCC & Breccia & HRDP & Hard pan & SBST & Sand and silt \\
\hline CLAY & Clay & LOAM & Loam & SGVC & Sand, gravel, and clay \\
\hline CLSD & Clay, some sand & MMPC & Metamorphic (undifferentiated) & SNCL & Sand, some clay \\
\hline COBB & Cobbles & MUCK & Muck & SCST & Schist \\
\hline COSD & Cobbles and sand & OTHR & Other & SDMN & Sedimentary (undifferentiated) \\
\hline DORT & Diorite & OBDN & Overburden & SILT & Silt \\
\hline GLCL & Glacial (undifferentiated) & PEAT & Peat & STCL & Silt and clay \\
\hline GRVL & Gravel & ROCK & Rock & SOIL & Soil \\
\hline GRCL & Gravel and clay & SAND & Sand & TILL & Till \\
\hline GRDS & Gravel, sand, and silt & SDCL & Sand and clay & & \\
\hline
\end{tabular}

The following abreviations are used to modify the lithologic descriptions:

$\begin{array}{ll}\text { VF } & \text { Very fine } \\ \text { F } & \text { Fine } \\ \text { M } & \text { Medium }\end{array}$


Table 3.--Lithologic logs of wells and borings

[ft, feet; --, no data available]

\begin{tabular}{|c|c|c|c|c|c|c|c|}
\hline \multicolumn{2}{|c|}{$\begin{array}{l}\text { Local } \\
\text { site } \\
\text { number }\end{array}$} & \multirow[t]{2}{*}{$\begin{array}{l}\text { Depth } \\
\text { drillied } \\
\text { (ft) }\end{array}$} & \multirow[t]{2}{*}{$\begin{array}{l}\text { Depth } \\
\text { of } \\
\text { well } \\
\text { (ft) }\end{array}$} & \multirow[t]{2}{*}{$\begin{array}{l}\text { Depth } \\
\text { to } \\
\text { refusal } \\
\text { (ft) }\end{array}$} & \multirow[t]{2}{*}{$\begin{array}{l}\text { Depth } \\
\text { to } \\
\text { top } \\
(\mathrm{ft})\end{array}$} & $\begin{array}{l}\text { Aquifer } \\
\text { code }\end{array}$ & \multirow[t]{2}{*}{ Iithology } \\
\hline \multicolumn{3}{|c|}{ ROCKINGHAM COUNTY } & & & & & \\
\hline BWA & 1 & 60 & -- & -- & $\begin{array}{r}0 \\
16 \\
42 \\
55 \\
59\end{array}$ & $\begin{array}{l}\text { 112NRSR } \\
\text { 112SRFD } \\
\text { 112MRIN } \\
\text { 112TILL } \\
\text { BEDROCK }\end{array}$ & $\begin{array}{l}\text { SAND BROWN, M-F } \\
\text { SDST WITH CLAY LAMINAE } \\
\text { STCL SOME SAND, LAMINATED } \\
\text { TILL SANDY, PEBBLEY } \\
\text { SCST F BIOTITE }\end{array}$ \\
\hline BWA & 2 & 43 & -- & -- & $\begin{array}{l}0 \\
12 \\
39.5\end{array}$ & $\begin{array}{l}\text { 112SRFD } \\
\text { 112SRFD } \\
\text { BEDROCK }\end{array}$ & $\begin{array}{l}\text { SAND F BROWN } \\
\text { STCL GRAY } \\
\text { SCST }\end{array}$ \\
\hline BWA & 4 & 50 & -- & 50 & $\begin{array}{r}0 \\
22 \\
47\end{array}$ & $\begin{array}{l}112 \text { SRFD } \\
112 \mathrm{GLCL} \\
112 \mathrm{TILL}\end{array}$ & $\begin{array}{l}\text { SDGL M-VC SAND, PEBBLES, BROWN } \\
\text { TILL SAND AND CLAY MATRIX }\end{array}$ \\
\hline BWA & 16 & 24 & -- & -- & $\begin{array}{r}1 \\
15\end{array}$ & $\begin{array}{l}\text { 112MRIN } \\
112 \text { MRIN }\end{array}$ & $\begin{array}{l}\text { SILT SOME CLAY } \\
\text { STCL -- }\end{array}$ \\
\hline BWA & 17 & 22 & 22 & 22 & $\begin{array}{r}0 \\
12 \\
17 \\
21\end{array}$ & $\begin{array}{l}112 \text { SRFD } \\
112 \text { MRIN } \\
112 \text { SRFD } \\
112 \text { TILI }\end{array}$ & $\begin{array}{l}\text { SAND E-M } \\
\text { STCL -- } \\
\text { SDGL SAND SOME GRAVEL } \\
\text { TILL }\end{array}$ \\
\hline BWS & 1 & -- & -- & -- & 0 & 112SRFD & SAND UNDER CLAY \\
\hline BWW & 1 & 40.5 & 40.5 & -- & $\begin{array}{l}1.5 \\
6.6 \\
20\end{array}$ & $\begin{array}{l}112 \mathrm{MRIN} \\
112 \text { SRFD } \\
112 \text { SRFD }\end{array}$ & $\begin{array}{l}\text { CLAY } \\
\text { SAND F SAND, SOME CLAY } \\
\text { SAND }\end{array}$ \\
\hline BWW & 2 & -- & 23 & -- & 0 & $112 \mathrm{MRIN}$ & SDCL AND SILT \\
\hline BWW & 3 & -- & 11.7 & -- & 0 & $112 \mathrm{MRIN}$ & SDCL AND SILT \\
\hline BWW & 4 & -- & 13.5 & -- & 0 & 112SRFD & SAND \\
\hline BWW & 5 & -- & 16.7 & -- & 0 & $112 \mathrm{TILL}$ & TILL \\
\hline BWW & 6 & -- & 168 & -- & -- & BEDROCK & \\
\hline BWW & 7 & -- & 250 & -- & 70 & BEDROCK & \\
\hline BWW & 8 & -- & 15.5 & -- & 0 & 112SRFD & SAND \\
\hline BWW & 9 & -- & 45 & -- & 0 & 112SRFD & SDGL \\
\hline
\end{tabular}


Table 3.--Lithologic logs of wells and borings--Continued

\begin{tabular}{|c|c|c|c|c|c|c|c|}
\hline \multicolumn{2}{|c|}{$\begin{array}{l}\text { Local } \\
\text { site } \\
\text { number }\end{array}$} & $\begin{array}{l}\text { Depth } \\
\text { drillled } \\
\text { (ft) }\end{array}$ & $\begin{array}{l}\text { Depth } \\
\text { of } \\
\text { well } \\
(f t)\end{array}$ & $\begin{array}{l}\text { Depth } \\
\text { to } \\
\text { refusal } \\
\text { (ft) }\end{array}$ & $\begin{array}{l}\text { Depth } \\
\text { to } \\
\text { top } \\
\text { (ft) }\end{array}$ & $\begin{array}{l}\text { Aquifer } \\
\text { code }\end{array}$ & Lithology \\
\hline & & & \multicolumn{5}{|c|}{ Brentwood--Cont inued } \\
\hline$B W W$ & 10 & 84 & 84 & -- & $\begin{array}{r}0 \\
24 \\
66\end{array}$ & $\begin{array}{l}\text { 112SRFD } \\
\text { 112SRFD } \\
\text { BEDROCK }\end{array}$ & $\begin{array}{l}\text { SDGL } \\
\text { SAND }\end{array}$ \\
\hline BWW & 11 & -- & 11.7 & -- & 0 & 112SRFD & SAND \\
\hline BWW & 12 & -- & 148 & -- & $\begin{array}{r}0 \\
96\end{array}$ & $\begin{array}{l}\text { 112TILL } \\
\text { BEDROCK }\end{array}$ & TILL HARDPAN \\
\hline BWW & 13 & -- & 31 & -- & 0 & 112SRFD & SDGL \\
\hline BWW & 14 & 110 & 110 & -- & 90 & BEDROCK & \\
\hline BWW & 15 & -- & 10.5 & -- & 0 & 112SRFD & SAND \\
\hline BWT & 19 & -- & 45 & -- & 0 & 112SRFD & \\
\hline BWW & 20 & 15 & 15 & -- & 0 & 1120TSH & \\
\hline BWW & 21 & 84 & 22 & -- & $\begin{array}{r}0 \\
12 \\
16 \\
32 \\
83\end{array}$ & $\begin{array}{l}112 \text { SRFD } \\
112 \text { SRFD } \\
112 \text { SRFD } \\
112 \text { SRFD } \\
112 \text { TILL }\end{array}$ & $\begin{array}{l}\text { SAND F } \\
\text { STCL WITH M SAND, LAMINATED } \\
\text { SNCL E-M SAND, WITH CLAY LAYERS } \\
\text { CLSD } \\
\text { TILL }\end{array}$ \\
\hline BWT & 22 & 51 & 50 & 51 & $\begin{array}{l}0 \\
26 \\
38 \\
47 \\
48 \\
48.3 \\
49\end{array}$ & $\begin{array}{l}112 \mathrm{NRSR} \\
112 \mathrm{NRSR} \\
112 \mathrm{SRFD} \\
112 \mathrm{NRSR} \\
112 \mathrm{MRIN} \\
112 \mathrm{TILL} \\
\text { BEDROCK }\end{array}$ & $\begin{array}{l}\text { SAND BROWN, M-F } \\
\text { SAND M-C, GRANULES, SOME F } \\
\text { SAND F-VE, LAMINATED } \\
\text { SDGL C, SAND, PEBBLES } \\
\text { SAND F, WITH CLAY LAMINAE } \\
\text { TILL } \\
\text { SCST F BIOTITE }\end{array}$ \\
\hline BWW & 26 & 68 & 55 & 68 & $\begin{array}{l}0 \\
10 \\
15 \\
30 \\
47 \\
52 \\
57\end{array}$ & $\begin{array}{l}112 \mathrm{NRSR} \\
112 \mathrm{NRSR} \\
112 \mathrm{NRSR} \\
112 \mathrm{MRIN} \\
112 \mathrm{SRFD} \\
112 \mathrm{SRFD} \\
112 \mathrm{SRFD}\end{array}$ & $\begin{array}{l}\text { SAND BROWN, M-C } \\
\text { SAND BROWN, F-VE } \\
\text { SDST V-VF, WITH CLAY } \\
\text { SILT GRAY, WITH CLAY } \\
\text { SAND F, SOME C } \\
\text { SDGL M-C, GRANULES, PEBBLES } \\
\text { SAND VF-C, LAYERS }\end{array}$ \\
\hline BWW & 27 & 45 & 12 & 45 & $\begin{array}{r}0 \\
12 \\
27\end{array}$ & $\begin{array}{l}112 \text { SRFD } \\
112 \text { SRFD } \\
112 \text { TILL }\end{array}$ & $\begin{array}{l}\text { SDGL } \\
\text { STCL WITH SAND, LAMINATED } \\
\text { TILL }\end{array}$ \\
\hline BWW & 28 & 76 & 32 & 76 & $\begin{array}{l}0 \\
32 \\
57 \\
69 \\
74 \\
76\end{array}$ & $\begin{array}{l}112 \text { SRFD } \\
112 \text { SRFD } \\
112 \text { SRFD } \\
112 \text { SRFD } \\
112 \text { TILL } \\
\text { BEDROCK }\end{array}$ & $\begin{array}{l}\text { SDGL WITH } 1 \text { FT SILT LAYER } \\
\text { GRDS } \\
\text { SAND VF-M, SOME C } \\
\text { SNCL } \\
\text { TILL }\end{array}$ \\
\hline BWW & 29 & 64 & 30 & 61 & $\begin{array}{l}0 \\
33 \\
40 \\
45 \\
61\end{array}$ & $\begin{array}{l}112 \text { SRFD } \\
112 \text { SRFD } \\
112 \text { SRFD } \\
112 \text { SRFD } \\
112 \text { TILL }\end{array}$ & $\begin{array}{l}\text { SDGL C-M SAND, WITH PEBBLES } \\
\text { SAND F-M, SOME SILT } \\
\text { SDGL F-VC SAND, SOME PEBBLES } \\
\text { STCL VF-F SAND } \\
\text { TILL }\end{array}$ \\
\hline BWW & 30 & 42 & 40 & -- & 0 & 112SRFD & SAND VF-C, SOME GRAVEL \\
\hline BWW & 31 & 55 & 55 & - & $\begin{array}{l}0 \\
-- \\
--\end{array}$ & $\begin{array}{l}110 \text { SDMN } \\
110 \text { SDMN } \\
110 \text { SDMN }\end{array}$ & $\begin{array}{l}\text { CLAY } \\
\text { SDGL } \\
\text { SAND }\end{array}$ \\
\hline
\end{tabular}


Table 3.--Lithologic logs of wells and borings--Continued

\begin{tabular}{|c|c|c|c|c|c|c|c|c|}
\hline \multicolumn{2}{|c|}{$\begin{array}{l}\text { Local } \\
\text { site } \\
\text { number }\end{array}$} & $\begin{array}{l}\text { Depth } \\
\text { drillied } \\
(f t)\end{array}$ & $\begin{array}{l}\text { Depth } \\
\text { of } \\
\text { well } \\
(\mathrm{ft})\end{array}$ & $\begin{array}{l}\text { Depth } \\
\text { to } \\
\text { refusal } \\
\text { (ft) }\end{array}$ & $\begin{array}{l}\text { Depth } \\
\text { to } \\
\text { top } \\
(f t)\end{array}$ & $\begin{array}{l}\text { Aquifer } \\
\text { code }\end{array}$ & Lithology & \\
\hline \multicolumn{9}{|c|}{ Brentwood--Continued } \\
\hline BWW & 31 & (Continued) & & & 55 & $\begin{array}{l}110 \text { SDMN } \\
\text { BEDROCK }\end{array}$ & SGVC & \\
\hline BWW & 32 & 30 & 30 & -- & $\frac{0}{30}$ & $\begin{array}{l}110 \text { SDMN } \\
110 \text { SDMN } \\
110 \text { SDMN } \\
\text { BEDROCK }\end{array}$ & $\begin{array}{l}\text { CLAY } \\
\text { SDGL } \\
\text { CLAY }\end{array}$ & \\
\hline BWW & 33 & 73 & 65 & -- & $\begin{array}{r}0 \\
33 \\
60 \\
65\end{array}$ & $\begin{array}{l}112 \mathrm{DELT} \\
112 \mathrm{DELT} \\
112 \mathrm{DELT} \\
112 \mathrm{DELT}\end{array}$ & $\begin{array}{l}\text { SDGL BROWN } \\
\text { SAND F-M } \\
\text { SDGL M AND C GRAVEL } \\
\text { SAND F }\end{array}$ & \\
\hline BWW & 35 & 90 & 90 & -- & $\begin{array}{l}0 \\
-- \\
-- \\
--\end{array}$ & $\begin{array}{l}110 \text { SDMN } \\
110 \text { SDMN } \\
110 \text { SDMN } \\
110 \text { SDMN }\end{array}$ & $\begin{array}{l}\text { SDGL } \\
\text { SDGL } \\
\text { SDGL } \\
\text { SDGL }\end{array}$ & \\
\hline BWW & 36 & 98 & 98 & -- & $\frac{0}{--}$ & $\begin{array}{l}110 \text { SDMN } \\
110 \text { SDMN } \\
110 S D M N \\
\text { BEDROCK }\end{array}$ & $\begin{array}{l}\text { SDGL } \\
\text { SAND } \\
\text { SDGL }\end{array}$ & \\
\hline BWW & 37 & 72 & 72 & -- & $\begin{array}{c}0 \\
-- \\
-- \\
--\end{array}$ & $\begin{array}{l}110 \text { SDMN } \\
110 \text { SDMN } \\
110 \text { SDMN } \\
110 \text { SDMN } \\
\text { BEDROCK }\end{array}$ & $\begin{array}{l}\text { SAND } \\
\text { CLAY } \\
\text { SAND } \\
\text { SDGL }\end{array}$ & \\
\hline BWW & 41 & 81 & 81 & -- & $\begin{array}{l}0 \\
-- \\
-- \\
-- \\
-- \\
--\end{array}$ & $\begin{array}{l}110 \text { SDMN } \\
110 S D M N \\
110 S D M N \\
110 S D M N \\
110 S D M N \\
110 S D M N \\
\text { BEDROCK }\end{array}$ & $\begin{array}{l}\text { SDGL } \\
\text { SDGL } \\
\text { SAND } \\
\text { SDGL } \\
\text { OTHR } \\
\text { SDGL }\end{array}$ & \\
\hline BWW & 42 & 79 & 79 & -- & $\begin{array}{l}0 \\
-- \\
-- \\
-- \\
-- \\
81\end{array}$ & $\begin{array}{l}110 \text { SDMN } \\
110 \text { SDMN } \\
110 \text { SDMN } \\
110 \text { SDMN } \\
110 \text { SDMN } \\
\text { BEDROCK }\end{array}$ & $\begin{array}{l}\text { SDGL } \\
\text { SDGL } \\
\text { SAND } \\
\text { SDGL } \\
\text { OTHR }\end{array}$ & \\
\hline BWW & 43 & 96 & 96 & -- & $\begin{array}{c}0 \\
-- \\
-- \\
-- \\
--\end{array}$ & $\begin{array}{l}110 \text { SDMN } \\
110 \text { SDMN } \\
110 \text { SDMN } \\
110 \text { SDMN } \\
110 S D M N \\
\text { BEDROCK }\end{array}$ & $\begin{array}{l}\text { SAND } \\
\text { SGVC } \\
\text { SDGL } \\
\text { SDGL } \\
\text { CLAY }\end{array}$ & \\
\hline
\end{tabular}


Table 3.--Lithologic logs of wells and borings--Continued

\begin{tabular}{|c|c|c|c|c|c|c|c|c|}
\hline \multicolumn{2}{|c|}{$\begin{array}{l}\text { Local } \\
\text { site } \\
\text { number }\end{array}$} & $\begin{array}{l}\text { Depth } \\
\text { drililed } \\
(f t)\end{array}$ & $\begin{array}{l}\text { Depth } \\
\text { of } \\
\text { wel1 } \\
\text { (ft) }\end{array}$ & $\begin{array}{l}\text { Depth } \\
\text { to } \\
\text { refusal } \\
\text { (ft) }\end{array}$ & $\begin{array}{l}\text { Depth } \\
\text { to } \\
\text { top } \\
(\mathrm{ft})\end{array}$ & $\begin{array}{l}\text { Aquifer } \\
\text { code }\end{array}$ & Lithology & \\
\hline & & & & \multicolumn{5}{|c|}{ Brentwood--Cont inued } \\
\hline BWW & 44 & 35 & 35 & -- & 0 & 112DELT & SDGL $M$ AND $C$, BROWN & \\
\hline BWW & 45 & 87 & 60 & -- & $\begin{array}{r}0 \\
27 \\
43 \\
60 \\
63 \\
66\end{array}$ & $\begin{array}{l}112 \text { DELT } \\
112 \text { DELT } \\
112 \text { DELT } \\
112 \text { DELT } \\
112 \text { DELT } \\
112 \text { DELT }\end{array}$ & $\begin{array}{l}\text { SDGL M AND C, BROWN } \\
\text { SDGL F-M, BROWN, SOME } \\
\text { SDGL M C, BROWN } \\
\text { SILT SOME SAND, GRAY } \\
\text { SAND F AND M, GRAY } \\
\text { SILT GRAY }\end{array}$ & SILT \\
\hline BWW & 46 & 47 & 47 & -- & $\begin{array}{r}0 \\
26 \\
45\end{array}$ & $\begin{array}{l}112 \text { DELT } \\
112 \text { DELT } \\
112 \text { DELT }\end{array}$ & $\begin{array}{l}\text { SDGL M AND C, BROWN } \\
\text { SILT BROWN } \\
\text { SDGL BROWN }\end{array}$ & \\
\hline BWW & 47 & 150 & 150 & -- & -- & BEDROCK & & \\
\hline BWW & 48 & 53 & 53 & -- & $\begin{array}{c}0 \\
-- \\
53\end{array}$ & $\begin{array}{l}110 \text { SDMN } \\
110 \text { SDMN } \\
110 \text { SDMN } \\
\text { BEDROCK }\end{array}$ & $\begin{array}{l}\text { SAND } \\
\text { CLAY } \\
\text { SGVC }\end{array}$ & \\
\hline BWW & 49 & 28 & 28 & -- & $\begin{array}{c}0 \\
29\end{array}$ & $\begin{array}{l}110 \text { SDMN } \\
110 \text { SDMN } \\
\text { BEDROCK }\end{array}$ & $\begin{array}{l}\text { SAND } \\
\text { SDGL }\end{array}$ & \\
\hline BWW & 50 & 39 & 39 & -- & $\begin{array}{l}0 \\
-- \\
--\end{array}$ & $\begin{array}{l}110 \text { SDMN } \\
110 \text { SDMN } \\
110 \text { SDMN }\end{array}$ & $\begin{array}{l}\text { SAND } \\
\text { CLAY } \\
\text { SGVC }\end{array}$ & \\
\hline BWW & 51 & 63 & 63 & -- & $\begin{array}{l}0 \\
-- \\
--\end{array}$ & $\begin{array}{l}110 \text { SDMN } \\
110 \text { SDMN } \\
110 \text { SDMN }\end{array}$ & $\begin{array}{l}\text { CLAY } \\
\text { SDGL } \\
\text { SGVC }\end{array}$ & \\
\hline BWW & 52 & 220 & 220 & -- & $\begin{array}{r}0 \\
14\end{array}$ & $\begin{array}{l}110 S D M N \\
\text { BEDROCK }\end{array}$ & CLAY & \\
\hline BWW & 53 & 550 & 550 & -- & $\begin{array}{r}0 \\
13\end{array}$ & $\begin{array}{l}110 \text { SDMN } \\
\text { BEDROCK }\end{array}$ & CLAY & \\
\hline BWW & 56 & 120 & 120 & -- & 20 & BEDROCK & & \\
\hline BWW & 57 & 300 & 300 & -- & $\begin{array}{c}0 \\
16.5\end{array}$ & $\begin{array}{l}110 \text { SDMN } \\
\text { BEDROCK }\end{array}$ & SDGL & \\
\hline BWW & 58 & 300 & 300 & -- & $\begin{array}{r}0 \\
22\end{array}$ & $\begin{array}{l}\text { 110SDMN } \\
\text { BEDROCK }\end{array}$ & SDGL & \\
\hline BWW & 59 & 275 & 275 & -- & $\begin{array}{l}0 \\
5\end{array}$ & $\begin{array}{l}110 \text { SDMN } \\
\text { BEDROCK }\end{array}$ & SAND & \\
\hline BWW & 60 & 260 & 260 & -- & $\begin{array}{c}0 \\
--\end{array}$ & $\begin{array}{l}110 S D M N \\
112 \text { TILL } \\
\text { BEDROCK }\end{array}$ & $\begin{array}{l}\text { SAND } \\
\text { TILL }\end{array}$ & \\
\hline BWW & 61 & 305 & 305 & -- & $\begin{array}{r}0 \\
25\end{array}$ & $\begin{array}{l}\text { 112TILL } \\
\text { BEDROCK }\end{array}$ & TILL & \\
\hline BWW & 62 & 175 & 175 & -- & $\begin{array}{r}0 \\
19\end{array}$ & $\begin{array}{l}110 \text { SDMN } \\
\text { BEDROCK }\end{array}$ & SAND & \\
\hline BWW & 63 & 750 & 750 & -- & $\begin{array}{r}0 \\
18\end{array}$ & $\begin{array}{l}110 \text { SDMN } \\
\text { BEDROCK }\end{array}$ & SDGL & \\
\hline BWW & 64 & 240 & 240 & -- & $\begin{array}{l}0 \\
5\end{array}$ & $\begin{array}{l}\text { 110SDMN } \\
\text { BEDROCK }\end{array}$ & SDGL & \\
\hline
\end{tabular}


Table 3.--Lithologic logs of wells and borings--Continued

\begin{tabular}{|c|c|c|c|c|c|c|c|}
\hline \multicolumn{2}{|c|}{$\begin{array}{l}\text { Local } \\
\text { site } \\
\text { number }\end{array}$} & $\begin{array}{l}\text { Depth } \\
\text { drilliled } \\
\quad(f t)\end{array}$ & $\begin{array}{l}\text { Depth } \\
\text { of } \\
\text { well } \\
\text { (ft) }\end{array}$ & $\begin{array}{l}\text { Depth } \\
\text { to } \\
\text { refusal } \\
\text { (ft) }\end{array}$ & $\begin{array}{l}\text { Depth } \\
\text { to } \\
\text { top } \\
\text { (ft) }\end{array}$ & $\begin{array}{l}\text { Aquifer } \\
\text { code }\end{array}$ & Lithology \\
\hline \multicolumn{8}{|c|}{ Brentwood--Cont inued } \\
\hline BWW & 65 & 650 & 650 & -- & $\begin{array}{c}0 \\
28\end{array}$ & $\begin{array}{l}110 \text { SDMN } \\
110 \text { SDMN } \\
\text { BEDROCK }\end{array}$ & $\begin{array}{l}\text { SAND } \\
\text { CLAY }\end{array}$ \\
\hline BWW & 66 & 100 & 100 & -- & $\begin{array}{c}0 \\
46 \\
80\end{array}$ & $\begin{array}{l}110 \text { SDMN } \\
110 \text { SDMN } \\
\text { BEDROCK } \\
110 \text { SDMN } \\
\text { BEDROCK }\end{array}$ & $\begin{array}{l}\text { SAND } \\
\text { CLAY } \\
\text { SAND }\end{array}$ \\
\hline BWW & 69 & 200 & 200 & -- & $\begin{array}{l}0 \\
6\end{array}$ & $\begin{array}{l}\text { 112TILL } \\
\text { BEDROCK }\end{array}$ & TILL \\
\hline BWW & 70 & 223 & 223 & -- & $\begin{array}{c}0 \\
85\end{array}$ & $\begin{array}{l}110 \text { SDMN } \\
110 \text { SDMN } \\
\text { BEDROCK }\end{array}$ & $\begin{array}{l}\text { SAND } \\
\text { CLAY }\end{array}$ \\
\hline BWW & 71 & 280 & 280 & -- & $\begin{array}{r}0 \\
10\end{array}$ & $\begin{array}{l}110 \text { SDMN } \\
\text { BEDROCK }\end{array}$ & SDGL \\
\hline BWW & 72 & 200 & 200 & - & $\frac{0}{40}$ & $\begin{array}{l}110 \text { SDMN } \\
110 \text { SDMN } \\
\text { BEDROCK }\end{array}$ & $\begin{array}{l}\text { SAND } \\
\text { CLAY }\end{array}$ \\
\hline BWW & 76 & 630 & 630 & -- & $\begin{array}{r}0 \\
151\end{array}$ & $\begin{array}{l}110 S D M N \\
\text { BEDROCK }\end{array}$ & SDGL \\
\hline BWW & 77 & 95 & 95 & -- & $\begin{array}{c}0 \\
-- \\
95\end{array}$ & $\begin{array}{l}110 \text { SDMN } \\
110 \text { SDMN } \\
\text { BEDROCK }\end{array}$ & $\begin{array}{l}\text { SGVC } \\
\text { CLAY }\end{array}$ \\
\hline BWW & 78 & 70 & 70 & -- & $\begin{array}{c}0 \\
-- \\
36\end{array}$ & $\begin{array}{l}110 S D M N \\
110 \text { SDMN } \\
\text { BEDROCK }\end{array}$ & $\begin{array}{l}\text { SAND } \\
\text { CLAY }\end{array}$ \\
\hline BWW & 79 & 160 & 160 & -- & $\begin{array}{l}0 \\
9\end{array}$ & $\begin{array}{l}\text { 112TILI } \\
\text { BEDROCK }\end{array}$ & TILL \\
\hline \multicolumn{8}{|c|}{ Candia } \\
\hline$C D B$ & 1 & 8 & 8 & -- & $\begin{array}{l}0 \\
8\end{array}$ & $\begin{array}{l}112 \text { SRFD } \\
\text { BEDROCK }\end{array}$ & GRVL \\
\hline $\mathrm{CDB}$ & 2 & 20 & 20 & - & $\begin{array}{r}0 \\
20\end{array}$ & $\begin{array}{l}\text { 112TILL } \\
\text { BEDROCK }\end{array}$ & TILL SANDY \\
\hline $\mathrm{CDB}$ & 5 & 25 & 25 & -- & $\begin{array}{r}0 \\
1 \\
25\end{array}$ & $\begin{array}{l}\text { 110SOIL } \\
112 \text { TILL } \\
\text { BEDROCK }\end{array}$ & \\
\hline
\end{tabular}


Table 3.--Lithologic logs of wells and borings--Continued

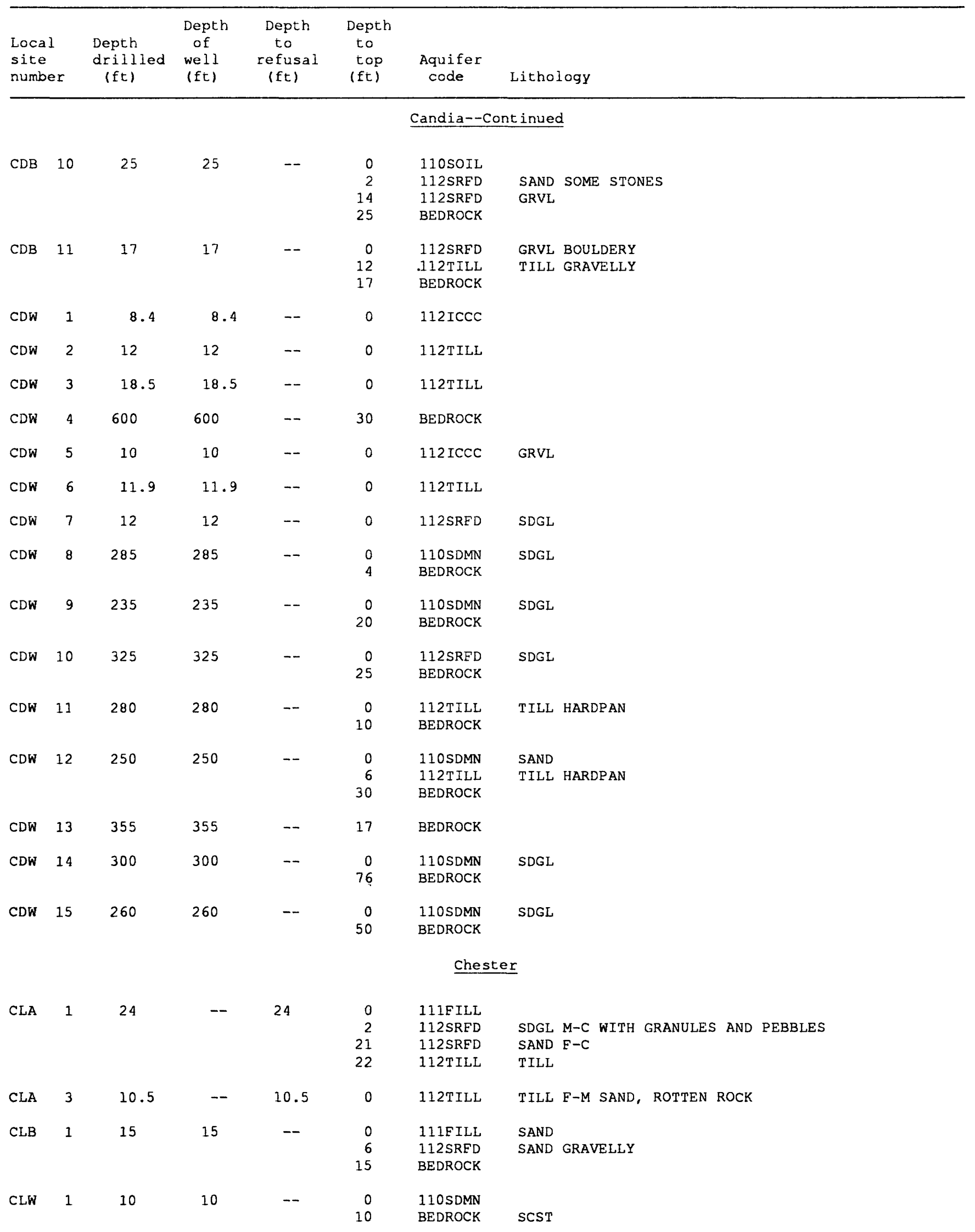


Table 3.--Lithologic logs of wells and borings--Continued

\begin{tabular}{|c|c|c|c|c|c|c|c|}
\hline \multicolumn{2}{|c|}{$\begin{array}{l}\text { Local } \\
\text { site } \\
\text { number }\end{array}$} & \multirow{3}{*}{$\begin{array}{l}\begin{array}{l}\text { Depth } \\
\text { drillied } \\
\text { (ft) }\end{array} \\
12\end{array}$} & \multirow{3}{*}{$\begin{array}{c}\begin{array}{c}\text { Depth } \\
\text { of } \\
\text { well } \\
(f t)\end{array} \\
\end{array}$} & \multirow{3}{*}{$\begin{array}{c}\begin{array}{c}\text { Depth } \\
\text { to } \\
\text { refusal } \\
(f t)\end{array} \\
\\
12\end{array}$} & \multirow{3}{*}{$\begin{array}{c}\begin{array}{c}\text { Depth } \\
\text { to } \\
\text { top } \\
(\mathrm{ft})\end{array} \\
\\
\\
\\
0 \\
8 \\
11\end{array}$} & $\begin{array}{l}\text { Aquifer } \\
\text { code }\end{array}$ & \multirow{2}{*}{ Lithology } \\
\hline & & & & & & Chester--c & \\
\hline CLW & 2 & & & & & $\begin{array}{l}112 \text { SRFD } \\
\text { 112SRFD } \\
\text { BEDROCK }\end{array}$ & $\begin{array}{l}\text { SDST F-M SAND, SOME SILT } \\
\text { SDST F-M SAND, SOME SILT } \\
\text { ROCK ROTTEN }\end{array}$ \\
\hline CLW & 3 & 250 & 250 & -- & $\begin{array}{l}0 \\
1\end{array}$ & $\begin{array}{l}110 \text { SDMN } \\
\text { BEDROCK }\end{array}$ & SCST \\
\hline CLW & 4 & 5.5 & -- & 5.5 & 0 & 112SRFD & SDST WITH ROTTEN ROCK \\
\hline CLW & 8 & 19.5 & 13.3 & 19.5 & $\begin{array}{r}0 \\
5 \\
14\end{array}$ & $\begin{array}{l}112 \text { SRFD } \\
112 \text { SRFD } \\
112 \mathrm{TILL}\end{array}$ & $\begin{array}{l}\text { SAND F OVER M } \\
\text { SDGL M-C SAND, F GRAVEL } \\
\text { TILL }\end{array}$ \\
\hline CLW & 9 & 34 & 28.5 & 34 & $\begin{array}{l}0 \\
16 \\
25 \\
29.5\end{array}$ & $\begin{array}{l}112 \text { SRFD } \\
112 \text { SRFD } \\
112 \text { SRFD } \\
112 \text { TILL }\end{array}$ & $\begin{array}{l}\text { SAND } M-C \text {, SOME GRANULES } \\
\text { SAND F, AND SILT } \\
\text { SAND F-M } \\
\text { TILL }\end{array}$ \\
\hline CLW & 10 & -- & 175 & -- & -- & BEDROCK & \\
\hline CLW & 11 & -- & 24 & -- & -- & -- & \\
\hline CLW & 13 & -- & 12 & -- & -- & -- & \\
\hline CLW & 14 & -- & 400 & -- & -- & BEDROCK & \\
\hline CLW & 15 & -- & 10.5 & -- & -- & -- & \\
\hline CLW & 17 & -- & 20 & -- & -- & -- & \\
\hline CLW & 18 & -- & 12 & -- & -- & -- & \\
\hline CLW & 19 & -- & 12 & -- & -- & -- & \\
\hline CLW & 20 & -- & 12 & -- & -- & -- & \\
\hline CLW & 21 & -- & 18 & -- & -- & -- & \\
\hline CLW & 22 & -- & 22 & -- & -- & -- & \\
\hline CLW & 23 & -- & 5 & -- & -- & -- & \\
\hline CLW & 24 & -- & 18 & -- & -- & -- & \\
\hline CLW & 25 & -- & 14.7 & -- & -- & -- & \\
\hline CLW & 26 & -- & 14 & -- & -- & -- & \\
\hline CLW & 28 & 10.9 & 10.9 & -- & -- & -- & \\
\hline CLW & 29 & -- & 250 & -- & -- & BEDROCK & \\
\hline CLW & 30 & -- & 70 & -- & -- & BEDROCK & \\
\hline CLW & 31 & -- & 12 & -- & -- & -- & \\
\hline CLW & 32 & -- & 15 & -- & -- & -- & \\
\hline CLW & 33 & -- & 250 & -- & -- & BEDROCK & \\
\hline CLW & 34 & -- & 16 & -- & -- & -- & \\
\hline CLW & 35 & -- & 28 & -- & -- & BEDROCK & \\
\hline
\end{tabular}


Table 3.--Lithologic logs of wells and borings--Continued

\begin{tabular}{lllllll}
\hline & \multicolumn{1}{c}{ Depth } & Depth & Depth & & \\
Local & Depth & of & to & to & & \\
site & drillled & well & refusal & top & Aquifer & \\
number & $(f t)$ & (ft) & (ft) & (ft) & code & Lithology
\end{tabular}

Chester--Continued

\begin{tabular}{|c|c|c|c|c|c|c|c|}
\hline CLW & 37 & -- & 25 & -- & -- & -- & \\
\hline CLW: & 38 & -- & 10 & -- & -- & -- & \\
\hline CLW & 39 & -- & 72 & -- & -- & BEDROCK & \\
\hline CLW & 40 & -- & 75 & -- & -- & BEDROCK & \\
\hline CLW & 41 & 19.5 & 19.5 & 19.5 & $\begin{array}{l}0 \\
5 \\
8.5 \\
13\end{array}$ & $\begin{array}{l}112 \text { SRFD } \\
112 \text { SRFD } \\
112 \text { SRFD } \\
112 \text { TILL }\end{array}$ & $\begin{array}{l}\text { SDST F-M SAND, SOME SOME SILT } \\
\text { SAND F-M } \\
\text { SDST F-M S } \\
\text { TILL F-M SAND, SILT, STONES }\end{array}$ \\
\hline CLW & 42 & 14.2 & 14.2 & 14.2 & $\begin{array}{r}0 \\
12\end{array}$ & $\begin{array}{l}111 F I L L \\
112 \text { TILL }\end{array}$ & $\begin{array}{l}\text { SAND F-M, TR C, TRASH } \\
\text { TILL F-M SAND, SOME SILT AND CLAY }\end{array}$ \\
\hline CLW & 43 & 13.5 & 13.5 & 13.5 & $\begin{array}{l}0 \\
8\end{array}$ & $\begin{array}{l}\text { 112SRFD } \\
112 \text { TILL }\end{array}$ & $\begin{array}{l}\text { SAND F-M, TR C } \\
\text { TILL F SAND, SILT, ROTTEN ROCK }\end{array}$ \\
\hline CLW & 44 & 9.5 & 9.5 & 9.5 & $\begin{array}{l}0 \\
7\end{array}$ & $\begin{array}{l}112 \mathrm{TILL} \\
112 \mathrm{TILL}\end{array}$ & $\begin{array}{l}\text { TILL SAND, SILT, ROTTEN ROCK } \\
\text { TILL SAND, SILT, CLAY }\end{array}$ \\
\hline CLW & 45 & 12 & 12 & 12 & 0 & 112SRFD & SDST F-M SAND, TRACE ORGANIC \\
\hline CLW & 46 & 15.5 & 15.5 & 15.5 & $\begin{array}{r}0 \\
7 \\
10\end{array}$ & $\begin{array}{l}112 \text { SRFD } \\
112 \text { SRFD } \\
112 \text { TILL }\end{array}$ & $\begin{array}{l}\text { SDST F-M SAND, BROWN } \\
\text { SDST F-C SAND, SOME SILT, GRAY } \\
\text { TILL SAND, SILT, CLAY }\end{array}$ \\
\hline CLW & 47 & 13.5 & 13.5 & 13.5 & $\begin{array}{l}0 \\
11.5\end{array}$ & $\begin{array}{l}\text { 112SRFD } \\
\text { 112TILL }\end{array}$ & $\begin{array}{l}\text { SDST F-M SAND, TRACE ORGANIC } \\
\text { TILI F SAND, SILT, GRAY }\end{array}$ \\
\hline CLW & 48 & 16 & 16 & 16 & 0 & 112SRFD & SDST F-M SAND, SOME SILT, BROWN \\
\hline CLW & 49 & 15 & 15 & 15 & 0 & 112SRFD & SAND $F-M$, BROWN \\
\hline CLW & 50 & \multicolumn{6}{|c|}{ Deerfield } \\
\hline DDA & 1 & 61 & -- & 61 & $\begin{array}{r}0 \\
26 \\
48\end{array}$ & $\begin{array}{l}112 \text { SRFD } \\
112 \text { SRFD } \\
112 \text { TILL }\end{array}$ & $\begin{array}{l}\text { SDGL WITH PEBBLES, COBBLES, GRANULES } \\
\text { SAND F-M, WITH GRAVEL AT } 47 \text { FT } \\
\text { TILL SANDY MATRIX }\end{array}$ \\
\hline DDB & 1 & 18 & 18 & -- & $\begin{array}{r}0 \\
18\end{array}$ & $\begin{array}{l}\text { 112SRED } \\
\text { BEDROCK }\end{array}$ & SAND GRAVELLY \\
\hline DDS & 1 & -- & -- & -- & 0 & $112 \mathrm{ICCC}$ & GRVL \\
\hline DDS & 2 & -- & -- & -- & 0 & $112 \mathrm{ICCC}$ & GRVI \\
\hline DDW & 1 & 62 & 59 & -- & $\begin{array}{r}0 \\
62\end{array}$ & $\begin{array}{l}112 \text { ICCC } \\
\text { BEDROCK }\end{array}$ & SDGL \\
\hline DDW & 2 & 11.3 & 11.3 & -- & 0 & 112TILL & \\
\hline DDW & 3 & 31.2 & 31.2 & -- & 0 & 112TILL & \\
\hline DDW & 4 & 21.7 & 21.7 & -- & 0 & $112 \mathrm{TILL}$ & GRVL \\
\hline DDW & 5 & 12 & 12 & -- & 0 & $112 \mathrm{ICCC}$ & SDGL \\
\hline DDW & 6 & 14.5 & 14.5 & -- & 0 & 112TILL & \\
\hline
\end{tabular}


Table 3.--Lithologic logs of wells and borings--Continued

\begin{tabular}{|c|c|c|c|c|c|c|c|}
\hline $\begin{array}{l}\text { Loca } \\
\text { site } \\
\text { numbe }\end{array}$ & & $\begin{array}{l}\text { Depth } \\
\text { drillled } \\
\quad(\mathrm{ft})\end{array}$ & $\begin{array}{l}\text { Depth } \\
\text { of } \\
\text { well } \\
\text { (ft) }\end{array}$ & $\begin{array}{l}\text { Depth } \\
\text { to } \\
\text { refusal } \\
\text { (ft) }\end{array}$ & $\begin{array}{l}\text { Depth } \\
\text { to } \\
\text { top } \\
(f t)\end{array}$ & $\begin{array}{l}\text { Aquifer } \\
\text { code }\end{array}$ & Lithology \\
\hline & & & & & & erfield- & ont inued \\
\hline DDW & 7 & -- & 208 & -- & 20 & BEDROCK & \\
\hline DDW & 10 & -- & 157 & -- & -- & BEDROCK & \\
\hline DDW & 11 & -- & 90 & -- & -- & BEDROCK & \\
\hline DDW & 12 & -- & 10 & -- & -- & -- & \\
\hline DDW & 16 & -- & 11 & -- & -- & -- & \\
\hline DDW & 18 & -- & 10 & -- & -- & -- & \\
\hline DDW & 20 & -- & 8.7 & -- & -- & -- & \\
\hline DDW & 21 & -- & 14 & -- & -- & -- & \\
\hline DDW & 22 & -- & 16 & -- & -- & -- & \\
\hline DDW & 24 & -- & 15 & -- & -- & -- & \\
\hline DDW & 26 & -- & 97 & -- & -- & -- & \\
\hline $\mathrm{DDW}$ & 32 & -- & 90 & -- & -- & BEDROCK & \\
\hline DDW & 33 & -- & 390 & -- & -- & BEDROCK & \\
\hline DDW & 34 & -- & 10.5 & -- & -- & -- & \\
\hline DDW & 36 & -- & 15 & -- & -- & -- & \\
\hline DDW & 37 & -- & 12 & -- & -- & -- & \\
\hline DDW & 38 & -- & 12 & -- & -- & -- & \\
\hline DDW & 40 & -- & 15 & -- & -- & -- & \\
\hline DDW & 41 & -- & 12 & -- & 12 & BEDROCK & \\
\hline DDW & 42 & -- & 20 & -- & -- & -- & \\
\hline DDW & 43 & -- & 260 & -- & -- & BEDROCK & \\
\hline DDW & 44 & 74 & 67.6 & -- & $\begin{array}{r}0 \\
5 \\
37 \\
62 \\
73\end{array}$ & $\begin{array}{l}\text { 112SRFD } \\
112 \text { SRFD } \\
112 \text { SRFD } \\
112 \text { SRFD } \\
\text { 112TILL }\end{array}$ & $\begin{array}{l}\text { SDGL SAND WITH PEBBLES } \\
\text { SAND } M-F \\
\text { SDST -- } \\
\text { SDGL C SAND WITH PEBBLES } \\
\text { TILL }\end{array}$ \\
\hline
\end{tabular}


Table 3.--Lithologic logs of wells and borings--Continued

\begin{tabular}{|c|c|c|c|c|c|c|c|}
\hline \multicolumn{2}{|c|}{$\begin{array}{l}\text { Local } \\
\text { site } \\
\text { number }\end{array}$} & $\begin{array}{l}\text { Depth } \\
\text { drililed } \\
\text { (ft) }\end{array}$ & $\begin{array}{l}\text { Depth } \\
\text { of } \\
\text { well } \\
(f t)\end{array}$ & $\begin{array}{l}\text { Depth } \\
\text { to } \\
\text { refusal } \\
\text { (ft) }\end{array}$ & $\begin{array}{l}\text { Depth } \\
\text { to } \\
\text { top } \\
\text { (ft) }\end{array}$ & $\begin{array}{l}\text { Aquifer } \\
\text { code }\end{array}$ & Lithology \\
\hline & & & & \multicolumn{4}{|c|}{ Deerfield--Continued } \\
\hline DDW & 45 & 18 & 14 & -- & $\begin{array}{l}0 \\
4 \\
17.5\end{array}$ & $\begin{array}{l}112 \text { SRFD } \\
112 \text { SRFD } \\
112 \text { TILL }\end{array}$ & $\begin{array}{l}\text { SDGL SAND, PEBBLES, COBBLES } \\
\text { SAND VC-M } \\
\text { TILL }\end{array}$ \\
\hline DDW & 46 & 70 & 47.5 & 70 & $\begin{array}{r}0 \\
29 \\
47\end{array}$ & $\begin{array}{l}112 \text { SRFD } \\
112 \text { SRFD } \\
112 \text { SRFD }\end{array}$ & $\begin{array}{l}\text { SDGL SAND WITH PEBBLES } \\
\text { SAND M-E } \\
\text { SDST -- }\end{array}$ \\
\hline DDW & 47 & 70 & 68.8 & 70 & $\begin{array}{r}0 \\
17 \\
27 \\
38 \\
68 \\
69\end{array}$ & $\begin{array}{l}11 \cdot 2 \text { SRFD } \\
112 \text { SRFD } \\
112 \text { SRFD } \\
112 \text { SRFD } \\
112 \text { SRFD } \\
112 \text { TILL }\end{array}$ & $\begin{array}{l}\text { SDGL C-F SAND, WITH PEBBLES } \\
\text { SAND } F \\
\text { SAND F, AND SILT } \\
\text { SAND VC-F } \\
\text { SDGL } \\
\text { TILL }\end{array}$ \\
\hline DDW & 48 & 377 & 377 & -- & $\begin{array}{l}0 \\
5\end{array}$ & $\begin{array}{l}112 \text { TILL } \\
\text { BEDROCK }\end{array}$ & TILL \\
\hline DDW & 49 & 316 & 316 & -- & $\begin{array}{r}0 \\
23\end{array}$ & $\begin{array}{l}112 \text { TILI } \\
\text { BEDROCK }\end{array}$ & TILL \\
\hline DDW & 50 & 300 & 300 & -- & $\begin{array}{l}0 \\
5\end{array}$ & $\begin{array}{l}110 \text { SDMN } \\
\text { BEDROCK }\end{array}$ & SDGL \\
\hline DDW & 51 & 300 & 300 & -- & $\begin{array}{l}0 \\
8\end{array}$ & $\begin{array}{l}\text { 110SDMN } \\
\text { BEDROCK }\end{array}$ & SDGL \\
\hline DDW & 52 & 295 & 295 & -- & $\begin{array}{l}0 \\
7\end{array}$ & $\begin{array}{l}\text { 112TILL } \\
\text { BEDROCK }\end{array}$ & TILL \\
\hline DDW & 53 & 340 & 340 & -- & $\begin{array}{r}0 \\
18\end{array}$ & $\begin{array}{l}110 S D M N \\
\text { BEDROCK }\end{array}$ & SDGL \\
\hline DDW & 54 & 295 & 295 & -- & $\begin{array}{l}0 \\
8\end{array}$ & $\begin{array}{l}\text { 112TILL } \\
\text { BEDROCK }\end{array}$ & TILL \\
\hline DDW & 55 & 360 & 360 & -- & $\begin{array}{r}0 \\
63\end{array}$ & $\begin{array}{l}110 \text { SDMN } \\
\text { BEDROCK }\end{array}$ & SDGL \\
\hline DDW & 56 & 190 & 190 & -- & $\begin{array}{r}0 \\
44\end{array}$ & $\begin{array}{l}110 S D M N \\
\text { BEDROCK }\end{array}$ & SAND \\
\hline DDW & 57 & 175 & 175 & -- & $\begin{array}{l}0 \\
5\end{array}$ & $\begin{array}{l}110 \text { SDMN } \\
\text { BEDROCK }\end{array}$ & SAND \\
\hline DDW & 58 & 155 & 155 & -- & $\begin{array}{l}0 \\
3.5\end{array}$ & $\begin{array}{l}112 \mathrm{TILL} \\
\text { BEDROCK }\end{array}$ & TILL \\
\hline DDW & 59 & 205 & 205 & -- & $\begin{array}{r}0 \\
20\end{array}$ & $\begin{array}{l}110 \text { SDMN } \\
\text { BEDROCK }\end{array}$ & SDGL \\
\hline DDW & 60 & 310 & 310 & -- & $\begin{array}{l}0 \\
8\end{array}$ & $\begin{array}{l}110 \text { SDMN } \\
\text { BEDROCK }\end{array}$ & SDGL \\
\hline DDW & 61 & 285 & 285 & -- & $\begin{array}{r}0 \\
12\end{array}$ & $\begin{array}{l}110 \text { SDMN } \\
\text { BEDROCK }\end{array}$ & SDGL \\
\hline
\end{tabular}


Table 3.--Lithologic logs of wells and borings--Continued

\begin{tabular}{|c|c|c|c|c|c|c|c|}
\hline \multicolumn{2}{|c|}{$\begin{array}{l}\text { Local } \\
\text { site } \\
\text { number }\end{array}$} & $\begin{array}{l}\text { Depth } \\
\text { drillied } \\
\text { (ft) }\end{array}$ & $\begin{array}{l}\text { Depth } \\
\text { of } \\
\text { well } \\
\text { (ft) }\end{array}$ & $\begin{array}{l}\text { Depth } \\
\text { to } \\
\text { refusal } \\
\text { (ft) }\end{array}$ & $\begin{array}{l}\text { Depth } \\
\text { to } \\
\text { top } \\
\text { (ft) }\end{array}$ & $\begin{array}{l}\text { Aquifer } \\
\text { code }\end{array}$ & Lithology \\
\hline \multicolumn{8}{|c|}{ Deerfield--Cont inued } \\
\hline DDW & 62 & 235 & 235 & -- & $\begin{array}{l}0 \\
7.5\end{array}$ & $\begin{array}{l}\text { 110SDMN } \\
\text { BEDROCK }\end{array}$ & SDGL \\
\hline DDW & 63 & 115 & 115 & -- & $\begin{array}{r}0 \\
23\end{array}$ & $\begin{array}{l}\text { 112TILL } \\
\text { BEDROCK }\end{array}$ & TILL \\
\hline DDW & 64 & 200 & 200 & -- & $\begin{array}{l}0 \\
6\end{array}$ & $\begin{array}{l}110 S D M N \\
\text { BEDROCK }\end{array}$ & SDGL \\
\hline \multicolumn{8}{|c|}{ East Kingston } \\
\hline EAW & 1 & -- & 14.3 & -- & 0 & 112 TILL & TILL \\
\hline EAW & 3 & 86 & 86 & -- & $\begin{array}{r}0 \\
50\end{array}$ & $\begin{array}{l}112 \text { TILL } \\
\text { BEDROCK }\end{array}$ & $\begin{array}{l}\text { TILL GRAVELLY, BOULDERY, WITH SILT } \\
\text { ROCK -- }\end{array}$ \\
\hline EAW & 4 & 62 & 62 & -- & $\begin{array}{r}0 \\
40\end{array}$ & $\begin{array}{l}\text { 112TILL } \\
\text { BEDROCK }\end{array}$ & $\begin{array}{l}\text { TILL SANDY TILI } \\
\text { ROCK -- }\end{array}$ \\
\hline EAW & 7 & 194 & 194 & -- & $\begin{array}{r}0 \\
92\end{array}$ & $\begin{array}{l}110 \text { SDMN } \\
\text { BEDROCK }\end{array}$ & $\begin{array}{l}\text { OTHR -- } \\
\text { ROCK -- }\end{array}$ \\
\hline EAW & 14 & 270 & 270 & -- & $\begin{array}{c}0 \\
--\end{array}$ & $\begin{array}{l}\text { 110SDMN } \\
\text { 112TILL } \\
\text { BEDROCK }\end{array}$ & $\begin{array}{l}\text { SDGL } \\
\text { TILI }\end{array}$ \\
\hline EAW & 15 & 260 & 260 & -- & $\begin{array}{r}0 \\
50\end{array}$ & $\begin{array}{l}11 \approx \text { TILL } \\
\text { BEDROCK }\end{array}$ & TILL \\
\hline EAW & 16 & 120 & 120 & -- & $\begin{array}{r}0 \\
40\end{array}$ & $\begin{array}{l}112 \text { TILL } \\
\text { BEDROCK }\end{array}$ & TILL \\
\hline EAW & 17 & 610 & 610 & -- & 65 & BEDROCK & \\
\hline EAW & 19 & 15 & 15 & -- & $\begin{array}{r}0 \\
15\end{array}$ & $\begin{array}{l}\text { 112TILL } \\
\text { BEDROCK }\end{array}$ & TILL \\
\hline EAW & 20 & 220 & 220 & -- & $\begin{array}{r}0 \\
45\end{array}$ & $\begin{array}{l}110 \text { SDMN } \\
\text { BEDROCK }\end{array}$ & CLAY \\
\hline EAW & 21 & 140 & 140 & -- & $\begin{array}{r}0 \\
96\end{array}$ & $\begin{array}{l}\text { 112TILL } \\
\text { BEDROCK }\end{array}$ & TILL \\
\hline
\end{tabular}


Table 3.--Lithologic logs of wells and borings--Continued

\begin{tabular}{|c|c|c|c|c|c|c|c|}
\hline \multicolumn{2}{|c|}{$\begin{array}{l}\text { Local } \\
\text { site } \\
\text { number }\end{array}$} & \multirow[t]{2}{*}{$\begin{array}{l}\text { Depth } \\
\text { drillled } \\
\text { (ft) }\end{array}$} & \multirow[t]{2}{*}{$\begin{array}{l}\text { Depth } \\
\text { of } \\
\text { well } \\
(f t)\end{array}$} & \multirow[t]{2}{*}{$\begin{array}{l}\text { Depth } \\
\text { to } \\
\text { refusal } \\
\text { (ft) }\end{array}$} & \multirow[t]{2}{*}{$\begin{array}{l}\text { Depth } \\
\text { to } \\
\text { top } \\
\text { (ft) }\end{array}$} & $\begin{array}{l}\text { Aquifer } \\
\text { code }\end{array}$ & \multirow[t]{2}{*}{ Lithology } \\
\hline \multicolumn{3}{|c|}{ Epping } & & & & & \\
\hline EPA & 1 & 43 & -- & 43 & $\begin{array}{r}0 \\
10 \\
33 \\
42\end{array}$ & $\begin{array}{l}112 \text { SRFD } \\
112 \text { SRFD } \\
112 \mathrm{MRIN} \\
112 \mathrm{TILL}\end{array}$ & $\begin{array}{l}\text { SDGL C-M SAND WITH GRANULES } \\
\text { SAND F-M WITH SILT LAYERS } \\
\text { CLAY } \\
\text { TILL F SAND MATRIX }\end{array}$ \\
\hline EPA & 2 & 20 & -- & 20 & $\begin{array}{r}0 \\
9 \\
15 \\
19\end{array}$ & $\begin{array}{l}112 \text { SRFD } \\
112 \mathrm{MRIN} \\
112 \mathrm{MRIN} \\
112 \mathrm{TILL}\end{array}$ & $\begin{array}{l}\text { SAND C, BROWN } \\
\text { CLAY GRAY } \\
\text { STCL } \\
\text { TILL }\end{array}$ \\
\hline EPA & 3 & 22.5 & -- & 22.5 & $\begin{array}{r}0 \\
2 \\
7 \\
17\end{array}$ & $\begin{array}{l}111 \mathrm{FILL} \\
112 \mathrm{SRFD} \\
112 \mathrm{MRIN} \\
112 \mathrm{MRIN}\end{array}$ & $\begin{array}{l}\text { SAND } \\
\text { SAND C-VC, BROWN } \\
\text { SILT WITH LAYER F SAND } \\
\text { STCL }\end{array}$ \\
\hline EPA & 4 & 44 & -- & 44 & $\begin{array}{r}0 \\
44\end{array}$ & $\begin{array}{l}112 \text { SRFD } \\
112 \text { SRFD }\end{array}$ & $\begin{array}{l}\text { SAND PEBBLES NEAR BOTTOM } \\
\text { GRVL PEBBLES }\end{array}$ \\
\hline EPA & 5 & 20 & 20 & -- & $\begin{array}{r}0 \\
10\end{array}$ & $\begin{array}{l}\text { 112SRFD } \\
112 \text { MRIN }\end{array}$ & $\begin{array}{l}\text { SAND M-C } \\
\text { STCL SOME SAND }\end{array}$ \\
\hline EPA & 6 & 26 & -- & 26 & $\begin{array}{r}0 \\
21\end{array}$ & $\begin{array}{l}112 \mathrm{MRIN} \\
112 \mathrm{TILL}\end{array}$ & $\begin{array}{l}\text { CLAY GRAY } \\
\text { TILL GRAVELLY, BROWN }\end{array}$ \\
\hline EPA & 7 & 21 & -- & 21 & $\begin{array}{l}0 \\
5 \\
12 \\
17.5\end{array}$ & $\begin{array}{l}112 \mathrm{NRSR} \\
112 \mathrm{MRIN} \\
112 \mathrm{MRIN} \\
\text { BEDROCK }\end{array}$ & $\begin{array}{l}\text { SAND VF, OVER SILT } \\
\text { SILT BROWN OVER GRAY } \\
\text { CLAY GRAY SILTY } \\
\text { ROCK BROKEN BEDROCK }\end{array}$ \\
\hline EPA & 10 & 19 & -- & 40 & $\begin{array}{r}0 \\
5 \\
10 \\
29\end{array}$ & $\begin{array}{l}112 \text { NRSR } \\
112 \text { MRIN } \\
112 \text { SRFD } \\
112 \text { TLL }\end{array}$ & $\begin{array}{l}\text { SAND M-F, SILTY } \\
\text { CLSD BLUE CLAY WITH F SAND } \\
\text { SNCL LAYERS } \\
\text { TILL }\end{array}$ \\
\hline EPA & 11 & 53 & -- & -- & $\begin{array}{l}0 \\
25.5 \\
49.5 \\
53\end{array}$ & $\begin{array}{l}\text { 112NRSR } \\
112 \text { MRIN } \\
112 \text { TILL } \\
\text { BEDROCK }\end{array}$ & $\begin{array}{l}\text { SAND F-M, BROWN } \\
\text { CLAY SILTY, TRACE SAND } \\
\text { TILL }\end{array}$ \\
\hline EPA & 12 & 41 & -- & -- & $\begin{array}{r}0 \\
2 \\
20\end{array}$ & $\begin{array}{l}111 \text { SOIL } \\
112 \text { NRSR } \\
112 \mathrm{MRIN}\end{array}$ & $\begin{array}{l}\text { SOIL TOPSOIL } \\
\text { GRVL, BROWN AND SILTY } \\
\text { CLAY SILTY }\end{array}$ \\
\hline EPA & 13 & 25 & -- & 25 & $\begin{array}{r}0 \\
20\end{array}$ & $\begin{array}{l}\text { 112NRSR } \\
112 \text { TILL }\end{array}$ & $\begin{array}{l}\text { SDGL } \\
\text { TILL GRAVELLY }\end{array}$ \\
\hline EPA & 14 & 17 & -- & 17 & $\begin{array}{r}0 \\
10\end{array}$ & $\begin{array}{l}\text { 112NRSR } \\
112 \mathrm{MRIN}\end{array}$ & $\begin{array}{l}\text { SDGL CLAYEY } \\
\text { CLAY GRAVELLY }\end{array}$ \\
\hline EPA & 17 & 31 & -- & 31 & $\begin{array}{r}0 \\
20 \\
25 \\
30\end{array}$ & $\begin{array}{l}112 \mathrm{MRIN} \\
112 \mathrm{GLCL} \\
112 \mathrm{SRFD} \\
112 \mathrm{GLCL}\end{array}$ & $\begin{array}{l}\text { CLAY WITH BROKEN ROCK } \\
\text { HARDPAN } \\
\text { GRVL CLAYEY } \\
\text { GLCL WITH BROKEN BEDROCK }\end{array}$ \\
\hline
\end{tabular}


Table 3.--Lithologic logs of wells and borings--Continued

\begin{tabular}{|c|c|c|c|c|c|c|c|c|}
\hline \multicolumn{2}{|c|}{$\begin{array}{l}\text { Local } \\
\text { site } \\
\text { number }\end{array}$} & \multirow{3}{*}{$\begin{array}{c}\begin{array}{l}\text { Depth } \\
\text { drillied } \\
\text { (ft) }\end{array} \\
\qquad 14\end{array}$} & \multirow{3}{*}{$\begin{array}{c}\begin{array}{c}\text { Depth } \\
\text { of } \\
\text { well } \\
(f t)\end{array} \\
--\end{array}$} & \multirow{3}{*}{$\begin{array}{l}\begin{array}{c}\text { Depth } \\
\text { to } \\
\text { refusa } 1 \\
\text { (ft) }\end{array} \\
\\
14\end{array}$} & \multirow{3}{*}{$\begin{array}{c}\text { Depth } \\
\text { to } \\
\text { top } \\
\text { (ft) }\end{array}$} & \multirow{2}{*}{$\begin{array}{l}\begin{array}{c}\text { Aquifer } \\
\text { code }\end{array} \\
\text { Epping--C }\end{array}$} & \multirow{2}{*}{\multicolumn{2}{|c|}{ Lithology }} \\
\hline & & & & & & & & \\
\hline EPA & 18 & & & & & 112MRIN & CLAY & WITH BROKEN ROCK \\
\hline EPA & 25 & 60 & - & -- & $\begin{array}{r}0 \\
15 \\
35\end{array}$ & $\begin{array}{l}112 \mathrm{NRSR} \\
112 \mathrm{MRIN} \\
112 \mathrm{MRIN}\end{array}$ & $\begin{array}{l}\text { SAND } \\
\text { CLAY } \\
\text { CLAY }\end{array}$ & $\begin{array}{l}\text { BROWN } \\
\text { GRAY } \\
\text { SANDY }\end{array}$ \\
\hline EPA & 35 & 78.7 & - & -- & $\begin{array}{r}0 \\
3 \\
10 \\
20 \\
32\end{array}$ & $\begin{array}{l}111 \text { SOIL } \\
112 \text { NRSR } \\
112 \text { NRSR } \\
112 \text { MRIN } \\
112 \text { MRIN }\end{array}$ & $\begin{array}{l}\text { SOIL } \\
\text { SAND } \\
\text { SAND } \\
\text { CLAY } \\
\text { CLAY }\end{array}$ & $\begin{array}{l}\text { TOPSOIL } \\
\text { BROWN } \\
\text { GRAY } \\
\text { SANDY, GRAY } \\
\text { GRAY }\end{array}$ \\
\hline EPA & 38 & 41 & -- & 41 & $\begin{array}{r}0 \\
20 \\
30\end{array}$ & $\begin{array}{l}112 \text { NRSR } \\
112 \mathrm{MRIN} \\
112 \mathrm{TILL}\end{array}$ & $\begin{array}{l}\text { SAND } \\
\text { CLAY } \\
\text { TILL }\end{array}$ & $\begin{array}{l}M-C \\
\text { GRAY }\end{array}$ \\
\hline EPA & 39 & 57 & -- & 57 & $\begin{array}{r}0 \\
10 \\
50 \\
55\end{array}$ & $\begin{array}{l}\text { 112NRSR } \\
112 \text { MRIN } \\
112 \text { MRIN } \\
112 \text { TILL }\end{array}$ & $\begin{array}{l}\text { SAND } \\
\text { CLAY } \\
\text { CLAY } \\
\text { TILL }\end{array}$ & $\begin{array}{l}\text { SILTY, BROWN } \\
\text { GRAY } \\
\text { SANDY, GRAY }\end{array}$ \\
\hline EPA & 41 & 74 & -- & 74 & $\begin{array}{r}0 \\
20 \\
55 \\
65\end{array}$ & $\begin{array}{l}112 \text { NRSR } \\
112 \text { MRIN } \\
112 \text { SRFD } \\
112 \text { SRFD }\end{array}$ & $\begin{array}{l}\text { SDGL } \\
\text { CLAY } \\
\text { SAND } \\
\text { SDGL }\end{array}$ & $\begin{array}{l}\text { C } \\
\text { GRAY } \\
\text { CLAYEY } \\
\text { M, GRAY }\end{array}$ \\
\hline EPA & 42 & 66 & -- & 66 & $\begin{array}{r}0 \\
20 \\
35 \\
65\end{array}$ & $\begin{array}{l}112 \text { NRSR } \\
112 \text { MRIN } \\
112 \text { SRFD } \\
112 \text { SRFD }\end{array}$ & $\begin{array}{l}\text { SDGL } \\
\text { CLAY } \\
\text { SAND } \\
\text { SAND }\end{array}$ & $\begin{array}{l}\text { C } \\
\text { GRAY } \\
\text { SILT AND CLAY } \\
\text { M, GRAY }\end{array}$ \\
\hline EPA & 43 & 74 & -- & 74 & $\begin{array}{r}0 \\
20 \\
50 \\
65\end{array}$ & $\begin{array}{l}\text { 112NRSR } \\
112 \mathrm{MRIN} \\
112 \mathrm{SRFD} \\
112 \mathrm{SRFD}\end{array}$ & $\begin{array}{l}\text { SDGL } \\
\text { CLAY } \\
\text { SAND } \\
\text { SDGL }\end{array}$ & $\begin{array}{l}\text { C } \\
\text { GRAY } \\
\text { SILTY AND CLAYEY } \\
\text { GRAY, M }\end{array}$ \\
\hline EPA & 44 & 56 & -- & 56 & $\begin{array}{r}0 \\
10\end{array}$ & $\begin{array}{l}\text { 112NRSR } \\
\text { 112MRIN }\end{array}$ & $\begin{array}{l}\text { SAND } \\
\text { CLAY }\end{array}$ & GRAY \\
\hline $\mathrm{EPA}$ & 45 & 10 & -- & -- & $\begin{array}{l}0 \\
1 \\
6\end{array}$ & $\begin{array}{l}111 \text { SWMP } \\
112 \mathrm{NRSR} \\
112 \mathrm{TILL}\end{array}$ & $\begin{array}{l}\text { PEAT } \\
\text { SDGL } \\
\text { TILL }\end{array}$ & CLAY, BROKEN STONES \\
\hline EPA & 46 & 13 & -- & -- & $\begin{array}{l}0 \\
1 \\
6\end{array}$ & $\begin{array}{l}111 \text { SWMP } \\
112 \text { NRSR } \\
112 \text { TILL }\end{array}$ & $\begin{array}{l}\text { PEAT } \\
\text { SDGL } \\
\text { TILL }\end{array}$ & CLAY, BROKEN STONES \\
\hline EPA & 47 & 14 & -- & -- & $\begin{array}{l}0 \\
1 \\
6\end{array}$ & $\begin{array}{l}111 \text { SWMP } \\
112 \text { NRSR } \\
112 \text { TILL }\end{array}$ & $\begin{array}{l}\text { PEAT } \\
\text { SDGL } \\
\text { TILL }\end{array}$ & CLAY AND BROKEN STONE \\
\hline EPA & 48 & 43 & -- & -- & $\begin{array}{r}0 \\
4 \\
40\end{array}$ & $\begin{array}{l}111 \text { SOIL } \\
112 \text { MRIN } \\
112 \text { SRFD }\end{array}$ & $\begin{array}{l}\text { SOIL } \\
\text { CLAY } \\
\text { GRVL }\end{array}$ & $\begin{array}{l}\text { TOPSOIL } \\
\text { RUNNY } \\
\text { SILTY }\end{array}$ \\
\hline$E P A$ & 57 & 26 & -- & -- & $\begin{array}{r}0 \\
20\end{array}$ & $\begin{array}{l}\text { 112NRSR } \\
\text { 112TILL }\end{array}$ & $\begin{array}{l}\text { SGVC } \\
\text { TILL }\end{array}$ & $\begin{array}{l}\text { BROWN } \\
\text { GRAY, CLAYEY }\end{array}$ \\
\hline EPA & 58 & 47 & -- & -- & $\begin{array}{r}0 \\
10 \\
20 \\
25 \\
35\end{array}$ & $\begin{array}{l}\text { 111SWMP } \\
112 \text { MRIN } \\
\text { 112MRIN } \\
112 \text { MRIN } \\
112 \text { TILL }\end{array}$ & $\begin{array}{l}\text { PEAT } \\
\text { CLAY } \\
\text { SILT } \\
\text { SILT } \\
\text { TILL }\end{array}$ & $\begin{array}{l}\text { BLUE } \\
\text { BROWN } \\
\text { GRAVELLY } \\
\text { GRAY, CLAYEY }\end{array}$ \\
\hline
\end{tabular}


Table 3.--Lithologic logs of wells and borings--Continued

\begin{tabular}{lllllll}
\hline & \multicolumn{1}{c}{ Depth } & Depth & Depth & \\
Local & Depth & of & to & to & & \\
site & drilled & well & refusal & top & Aquifer & \\
number & $(f t)$ & $(f t)$ & $(f t)$ & (ft) & code & Lithology \\
\hline
\end{tabular}

Epping--Cont inued

\begin{tabular}{|c|c|c|c|c|c|c|c|}
\hline EPA & 59 & 19 & -- & -- & $\begin{array}{r}0 \\
12\end{array}$ & $\begin{array}{l}\text { 112NRSR } \\
112 \text { TILL }\end{array}$ & $\begin{array}{l}\text { SDGL } \\
\text { TILI }\end{array}$ \\
\hline EPA & 60 & 13 & -- & -- & 0 & $112 \mathrm{GLCL}$ & OBDN BOULDERS \\
\hline EPA & 61 & 10 & -- & -- & 0 & $112 \mathrm{GLCL}$ & OBDN BOULDERS \\
\hline EPA & 62 & 10 & -- & -- & 0 & $112 \mathrm{GLCL}$ & OBDN BOULDERS \\
\hline EPA & 63 & 14 & -- & -- & 0 & $112 \mathrm{GLCL}$ & OBDN BOULDERS \\
\hline EPA & 64 & 16 & -- & -- & 0 & $112 \mathrm{GLCL}$ & OBDN BOULDERS \\
\hline EPA & 65 & 75 & -- & 75 & $\begin{array}{r}0 \\
10 \\
20 \\
35 \\
60\end{array}$ & $\begin{array}{l}112 \text { NRSR } \\
112 \text { NRSR } \\
112 \text { MRIN } \\
112 \text { TILI } \\
112 \text { SRFD }\end{array}$ & $\begin{array}{l}\text { SAND } \mathrm{F} \\
\text { SAND SILTY } \\
\text { CLAY SAND AND SILT } \\
\text { TILL CLAYEY } \\
\text { SAND CLAYEY }\end{array}$ \\
\hline EPA & 67 & 42 & -- & -- & $\begin{array}{l}0 \\
3\end{array}$ & $\begin{array}{l}111 \text { SOIL } \\
112 \text { NRSR }\end{array}$ & $\begin{array}{l}\text { SOIL TOPSOIL } \\
\text { GRVL BROWN AND SHARP }\end{array}$ \\
\hline EPA & 74 & 49 & -- & -- & $\begin{array}{l}0 \\
10 \\
21 \\
29.5\end{array}$ & $\begin{array}{l}112 \text { SRFD } \\
112 \text { SRFD } \\
112 \text { SRFD } \\
112 \mathrm{~T} \text { ILL }\end{array}$ & $\begin{array}{l}\text { SAND } M-C, \text { PEBBLE } \\
\text { SAND F-V F, BROWN } \\
\text { SDST VF-M. SAND, SILT AND CLAY } \\
\text { TILL SILTY - VERY SANDY MATRIX }\end{array}$ \\
\hline EPB & 1 & 12 & -- & 12 & 0 & 112NRSR & SDGL SILTY \\
\hline EPB & 2 & 22.5 & -- & 22.5 & 0 & 112NRSR & SAND STONEY \\
\hline $\mathrm{EPB}$ & 3 & 46 & -- & 46 & $\begin{array}{r}0 \\
4 \\
13 \\
36\end{array}$ & $\begin{array}{l}111 F I L L \\
112 \text { NRSR } \\
112 \mathrm{MRIN} \\
112 \text { SRFD }\end{array}$ & $\begin{array}{l}\text { SDGL GRAVEL } \\
\text { SAND F } \\
\text { CLAY } \\
\text { SAND GRAVELLY AND SANDY }\end{array}$ \\
\hline EPB & 4 & 6.6 & -- & 6.6 & $\begin{array}{l}0 \\
3.6 \\
5.9\end{array}$ & $\begin{array}{l}111 \mathrm{FILL} \\
112 \mathrm{NRSR} \\
112 \mathrm{TILL}\end{array}$ & $\begin{array}{l}\text { GRVL } \\
\text { SDGL C } \\
\text { TILL SAND AND GRAVEL }\end{array}$ \\
\hline EPB & 5 & 47.5 & -- & 47.5 & $\begin{array}{l}0 \\
13 \\
32 \\
39.3 \\
42\end{array}$ & $\begin{array}{l}112 \text { NRSR } \\
112 \mathrm{MRIN} \\
112 \mathrm{SRFD} \\
112 \mathrm{GLCL} \\
112 \mathrm{TILL}\end{array}$ & $\begin{array}{l}\text { SDGL SILTY } \\
\text { CLAY } \\
\text { SAND SILTY, STONEY } \\
\text { BLDR } \\
\text { TILL }\end{array}$ \\
\hline EPS & 1 & -- & -- & -- & 0 & 112SRFD & SAND OVER MARINE DEPOSITS \\
\hline EPW & 1 & -- & 9.7 & -- & 0 & 112SRFD & SAND \\
\hline EPW & 2 & 64 & 64 & -- & $\begin{array}{r}7 \\
35 \\
42 \\
59\end{array}$ & $\begin{array}{l}112 \mathrm{MRIN} \\
112 \mathrm{SRFD} \\
112 \mathrm{MRIN} \\
112 \mathrm{SRFD}\end{array}$ & $\begin{array}{l}\text { CLAY } \\
\text { SAND } \\
\text { CLAY } \\
\text { SDGL C SAND, F GRAVEL }\end{array}$ \\
\hline EPW & 3 & -- & 15.5 & -- & 0 & 112TILL & TILL \\
\hline EPW & 6 & 60 & 60 & -- & $\begin{array}{r}0 \\
3 \\
57 \\
60\end{array}$ & $\begin{array}{l}111 \mathrm{FILL} \\
112 \mathrm{MRIN} \\
112 \mathrm{SRFD} \\
\text { BEDROCK }\end{array}$ & $\begin{array}{l}\text { GRVL } \text { BROWN } \\
\text { CLAY GRAY } \\
\text { GRVL M BROWN } \\
\text { SDMN }\end{array}$ \\
\hline
\end{tabular}


Table 3.--Lithologic logs of wells and borings--Continued

\begin{tabular}{|c|c|c|c|c|c|c|c|c|}
\hline \multicolumn{2}{|c|}{$\begin{array}{l}\text { Local } \\
\text { site } \\
\text { number }\end{array}$} & $\begin{array}{l}\text { Depth } \\
\text { drillled } \\
\quad(f t)\end{array}$ & $\begin{array}{l}\text { Depth } \\
\text { of } \\
\text { well } \\
\text { (ft) }\end{array}$ & $\begin{array}{l}\text { Depth } \\
\text { to } \\
\text { refusal } \\
\text { (ft) }\end{array}$ & $\begin{array}{l}\text { Depth } \\
\text { to } \\
\text { top } \\
(f t)\end{array}$ & $\begin{array}{l}\text { Aquifer } \\
\text { code }\end{array}$ & Lithol & $\log y$ \\
\hline & & & & \multicolumn{5}{|c|}{ Epping--Continued } \\
\hline EPW & 7 & -- & 78 & -- & $\begin{array}{r}0 \\
18 \\
35 \\
60\end{array}$ & $\begin{array}{l}\text { 112OTSH } \\
112 \mathrm{MRIN} \\
112 \mathrm{MRIN} \\
112 \text { SRFD }\end{array}$ & $\begin{array}{l}\text { SAND } \\
\text { CLAY } \\
\text { CLSD } \\
\text { SAND }\end{array}$ & $\begin{array}{l}\text { GRAY, LIGHT } \\
\text { GRAY, LIGHT } \\
\text { F-M, BROWN, CLAY }\end{array}$ \\
\hline EPW & 8 & 78 & 78 & -- & 0 & 1120TSH & & \\
\hline EPW & 9 & 400 & 400 & -- & 50 & BEDROCK & & \\
\hline EPW & 10 & 15 & 15 & -- & 0 & 1120TSH & & \\
\hline EPW & 11 & 250 & 250 & -- & 18 & BEDROCK & & \\
\hline EPW & 13 & 20 & 20 & -- & $\begin{array}{r}0 \\
20\end{array}$ & $\begin{array}{l}1120 \mathrm{TSH} \\
112 \mathrm{MRIN}\end{array}$ & $\begin{array}{l}\text { SAND } \\
\text { STCL }\end{array}$ & \\
\hline EPW & 14 & 12 & 12 & -- & 0 & 112TILL & TILL & SANDY \\
\hline EPW & 15 & -- & 190 & -- & 15 & BEDROCK & MMPC & -- \\
\hline EPW & 16 & -- & 170 & -- & 58 & BEDROCK & MMPC & -- \\
\hline EPW & 17 & -- & 100 & -- & 16 & BEDROCK & MMPC & -- \\
\hline EPW & 18 & 26 & 26 & -- & $\begin{array}{r}0 \\
21 \\
22\end{array}$ & $\begin{array}{l}\text { 112MRIN } \\
112 \text { SRFD } \\
112 \text { SRFD }\end{array}$ & $\begin{array}{l}\text { CLAY } \\
\text { GRVL } \\
\text { GRVL }\end{array}$ & $\begin{array}{l}\text { GRAY } \\
\text { BROWN, BROKEN } \\
\text { C, BROWN, PACKED }\end{array}$ \\
\hline EPW & 19 & 41 & 37 & -- & -- & -- & & \\
\hline EPW & 20 & 41 & 36 & -- & $\begin{array}{r}0 \\
5 \\
20 \\
30 \\
35\end{array}$ & $\begin{array}{l}111 \text { SOIL } \\
112 \text { SRFD } \\
112 \text { SRFD } \\
112 \text { SRFD } \\
112 \text { SRFD }\end{array}$ & $\begin{array}{l}\text { GRVL } \\
\text { SILT } \\
\text { GRVL } \\
\text { SILT }\end{array}$ & $\begin{array}{l}\text { BROKEN, GRAY, SILT } \\
\text { BROWN } \\
\text { BROWN } \\
\text { RED, CLAY, BROWN }\end{array}$ \\
\hline EPW & 21 & 42 & 36 & -- & $\begin{array}{r}0 \\
5 \\
30 \\
35\end{array}$ & $\begin{array}{l}111 \text { SOIL } \\
112 \text { SRFD } \\
112 \text { SRFD } \\
112 \text { SRFD }\end{array}$ & $\begin{array}{l}\text { GRVL } \\
\text { GRVL } \\
\text { SILT }\end{array}$ & $\begin{array}{l}\text { BROKEN, GRAY } \\
\text { BROWN } \\
\text { RED， CLAY， GRAY }\end{array}$ \\
\hline EPW & 22 & 46 & 41 & -- & $\begin{array}{r}0 \\
20 \\
30 \\
35\end{array}$ & $\begin{array}{l}112 \mathrm{SRFD} \\
112 \mathrm{SRED} \\
112 \mathrm{SRFD} \\
112 \mathrm{SRFD}\end{array}$ & $\begin{array}{l}\text { GRVL } \\
\text { GRVL } \\
\text { GRVL } \\
\text { GRVL }\end{array}$ & $\begin{array}{l}\text { BROWN } \\
\text { RED } \\
\text { BROKEN, SHARP } \\
\text { BROWNISH }\end{array}$ \\
\hline EPW & 23 & -- & 100 & -- & 55 & BEDROCK & MMPC & -- \\
\hline EPW & 24 & -- & 145 & -- & 56 & BEDROCK & MMPC & -- \\
\hline EPW & 25 & -- & 205 & -- & 62 & BEDROCK & MMPC & -- \\
\hline EPW & 26 & -- & 270 & -- & 25 & BEDROCK & MMPC & -- \\
\hline EPW & 27 & -- & 140 & -- & 35 & BEDROCK & MMPC & -- \\
\hline EPW & 28 & 57 & 56 & 57 & $\begin{array}{r}0 \\
10 \\
50\end{array}$ & $\begin{array}{l}11 \angle \mathrm{NRSR} \\
112 \mathrm{MRIN} \\
112 \mathrm{TILL}\end{array}$ & $\begin{array}{l}\text { SAND } \\
\text { CLAY } \\
\text { TILL }\end{array}$ & $\begin{array}{l}\text { SILTY } \\
\text { GRAY } \\
\text { SANDY, GRAY }\end{array}$ \\
\hline EPW & 29 & -- & 208 & -- & 103 & BEDROCK & MMPC & -- \\
\hline EPW & 30 & -- & 104 & -- & 16 & BEDROCK & MMPC & -- \\
\hline
\end{tabular}


Table 3.--Lithologic logs of wells and borings--Continued

\begin{tabular}{|c|c|c|c|c|c|c|c|c|}
\hline \multicolumn{2}{|c|}{$\begin{array}{l}\text { Local } \\
\text { site } \\
\text { number }\end{array}$} & \multirow{3}{*}{$\begin{array}{c}\begin{array}{c}\text { Depth } \\
\text { drilliled } \\
(\mathrm{ft})\end{array} \\
\quad 34\end{array}$} & \multirow{3}{*}{$\begin{array}{c}\begin{array}{c}\text { Depth } \\
\text { of } \\
\text { well } \\
(\mathrm{ft})\end{array} \\
\\
\\
\end{array}$} & \multirow{3}{*}{$\begin{array}{c}\begin{array}{c}\text { Depth } \\
\text { to } \\
\text { refusal } \\
\text { (ft) }\end{array} \\
\\
--\end{array}$} & \multirow{2}{*}{$\begin{array}{l}\text { Depth } \\
\text { to } \\
\text { top } \\
\text { (ft) }\end{array}$} & $\begin{array}{l}\text { Aquifer } \\
\text { code }\end{array}$ & \multicolumn{2}{|c|}{ Lithology } \\
\hline & & & & & & Epping--c & tinued & \\
\hline EPW & 31 & & & & $\begin{array}{r}0 \\
5 \\
10 \\
26\end{array}$ & $\begin{array}{l}111 \text { SOIL } \\
112 \text { OTSH } \\
112 \mathrm{MRIN} \\
112 \text { SRFD }\end{array}$ & $\begin{array}{l}\text { GRVL } \\
\text { CLAY } \\
\text { GRVL }\end{array}$ & $\begin{array}{l}\text { GRAY } \\
\text { BROKEN, TRACE CLAY }\end{array}$ \\
\hline EPW & 32 & -- & 80 & -- & 11 & BEDROCK & MMPC & -- \\
\hline EPW & 33 & -- & 175 & -- & 36 & BEDROCK & MMPC & -- \\
\hline EPW & 34 & -- & 129 & -- & 15 & BEDROCK & MMPC & -- \\
\hline EPW & 35 & -- & 325 & -- & 62 & BEDROCK & MMPC & -- \\
\hline EPW & 36 & -- & 204 & -- & 56 & BEDROCK & MMPC & -- \\
\hline EPW & 37 & -- & 230 & -- & 145 & BEDROCK & MMPC & -- \\
\hline EPW & 38 & -- & 147 & -- & 131 & BEDROCK & MMPC & -- \\
\hline EPW & 39 & -- & 400 & -- & 41 & BEDROCK & MMPC & -- \\
\hline EPW & 40 & -- & 105 & -- & 60 & BEDROCK & MMPC & -- \\
\hline EPW & 41 & -- & 150 & -- & 15 & BEDROCK & MMPC & -- \\
\hline EPW & 42 & -- & 190 & -- & 15 & BEDROCK & MMPC & -- \\
\hline EPW & 43 & -- & 195 & -- & 14 & BEDROCK & MMPC & -- \\
\hline EPW & 45 & -- & 370 & -- & 24 & BEDROCK & MMPC & -- \\
\hline EPW & 46 & -- & 250 & -- & 66 & BEDROCK & MMPC & -- \\
\hline EPW & 47 & 55 & 55 & -- & $\begin{array}{r}0 \\
14 \\
52\end{array}$ & $\begin{array}{l}1120 T S H \\
112 \mathrm{MRIN} \\
112 \mathrm{SRFD}\end{array}$ & $\begin{array}{l}\text { SAND } \\
\text { CLAY } \\
\text { SAND }\end{array}$ & $\begin{array}{l}\text { BROWN } \\
\text { LIGHT GRAY } \\
\text { F, LIGHT GRAY }\end{array}$ \\
\hline EPW & 48 & 57 & 56 & 57 & $\begin{array}{r}0 \\
10 \\
50 \\
55\end{array}$ & $\begin{array}{l}112 \mathrm{NRSR} \\
112 \mathrm{MRIN} \\
112 \mathrm{MRIN} \\
112 \mathrm{TILL}\end{array}$ & $\begin{array}{l}\text { SAND } \\
\text { CLAY } \\
\text { CLSD } \\
\text { SAND }\end{array}$ & $\begin{array}{l}\text { SILTY } \\
\text { GRAY } \\
\text { M， GRAY， HARDPAN }\end{array}$ \\
\hline EPW & 49 & 43 & 43 & -- & $\begin{array}{r}0 \\
5 \\
10 \\
36\end{array}$ & $\begin{array}{l}112 \mathrm{MRIN} \\
112 \mathrm{OTSH} \\
112 \mathrm{MRIN} \\
112 \mathrm{SRFD}\end{array}$ & $\begin{array}{l}\text { CLAY } \\
\text { GRVL } \\
\text { CLAY } \\
\text { GRVL }\end{array}$ & $\begin{array}{l}\text { GRAY, TOPSOIL } \\
\text { BROWN } \\
\text { GRAY, RUNNEY } \\
\text { BROKEN, TRACE CLAY }\end{array}$ \\
\hline EPW & 50 & 31 & 31 & -- & $\begin{array}{r}0 \\
5 \\
10 \\
26\end{array}$ & $\begin{array}{l}\text { 111SOIL } \\
112 \text { SRFD } \\
112 \text { MRIN } \\
112 \text { SRFD }\end{array}$ & $\begin{array}{l}\text { GRVL } \\
\text { CLAY } \\
\text { GRVL }\end{array}$ & $\begin{array}{l}\text { BROWN } \\
\text { GRAY } \\
\text { BROWN }\end{array}$ \\
\hline EP.W & 51 & 78 & -- & -- & -- & -- & & \\
\hline EPW & 52 & 78 & -- & -- & -- & -- & & \\
\hline EPW & 53 & 82 & -- & -- & -- & -- & & \\
\hline EPW & 54 & 71 & -- & -- & -- & -- & & \\
\hline EPW & 55 & 99 & 20.3 & -- & $\begin{array}{l}0 \\
20.5 \\
29 \\
55.5 \\
95.5\end{array}$ & $\begin{array}{l}112 \mathrm{NRSR} \\
112 \mathrm{NRSR} \\
112 \mathrm{SRFD} \\
112 \mathrm{MRIN} \\
112 \mathrm{TILL}\end{array}$ & $\begin{array}{l}\text { SDGL } \\
\text { SAND } \\
\text { SDCL } \\
\text { STCL } \\
\text { TILL }\end{array}$ & $\begin{array}{l}\text { VC-M SAND - PEBBLES } \\
\text { F-VF, BROWN } \\
\text { VF-M, SILTY, GRAY } \\
\text { TRACE SAND LAYERS } \\
\text { SILT, F SAND MATRIX }\end{array}$ \\
\hline
\end{tabular}


Table 3.--Lithologic logs of wells and borings--Continued

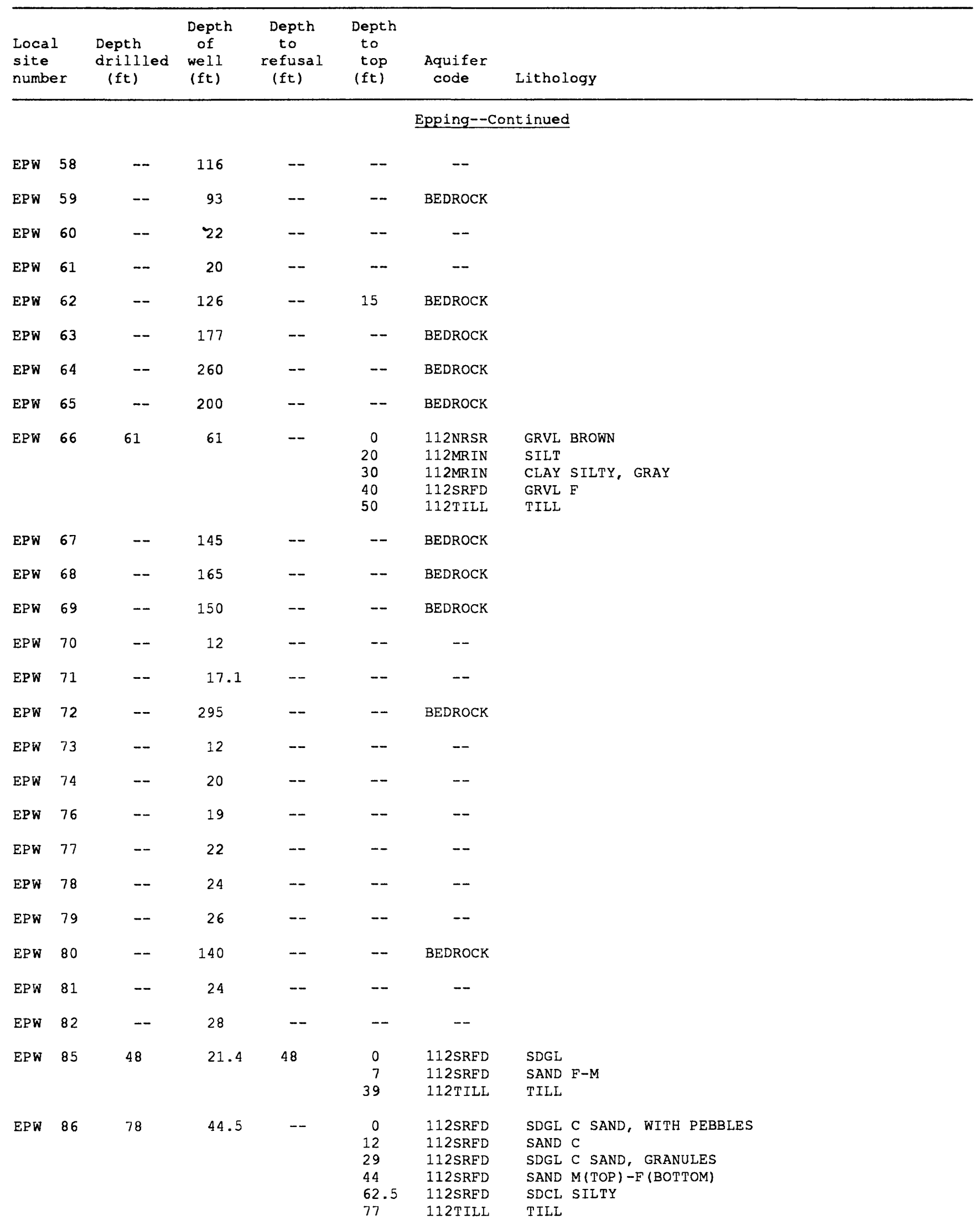


Table 3.--Lithologic logs of wells and borings--Continued

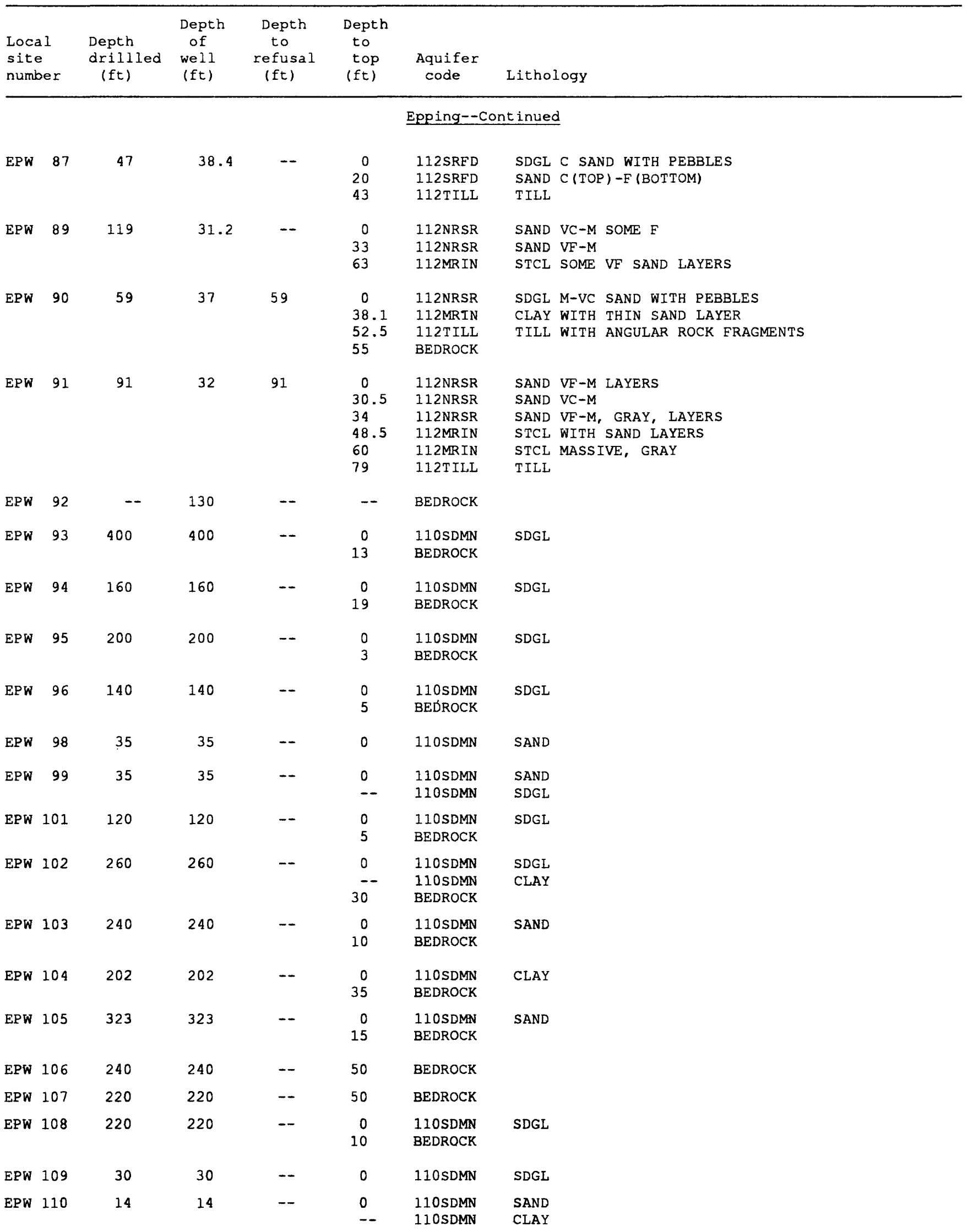


Table 3.--Lithologic logs of wells and borings--Continued

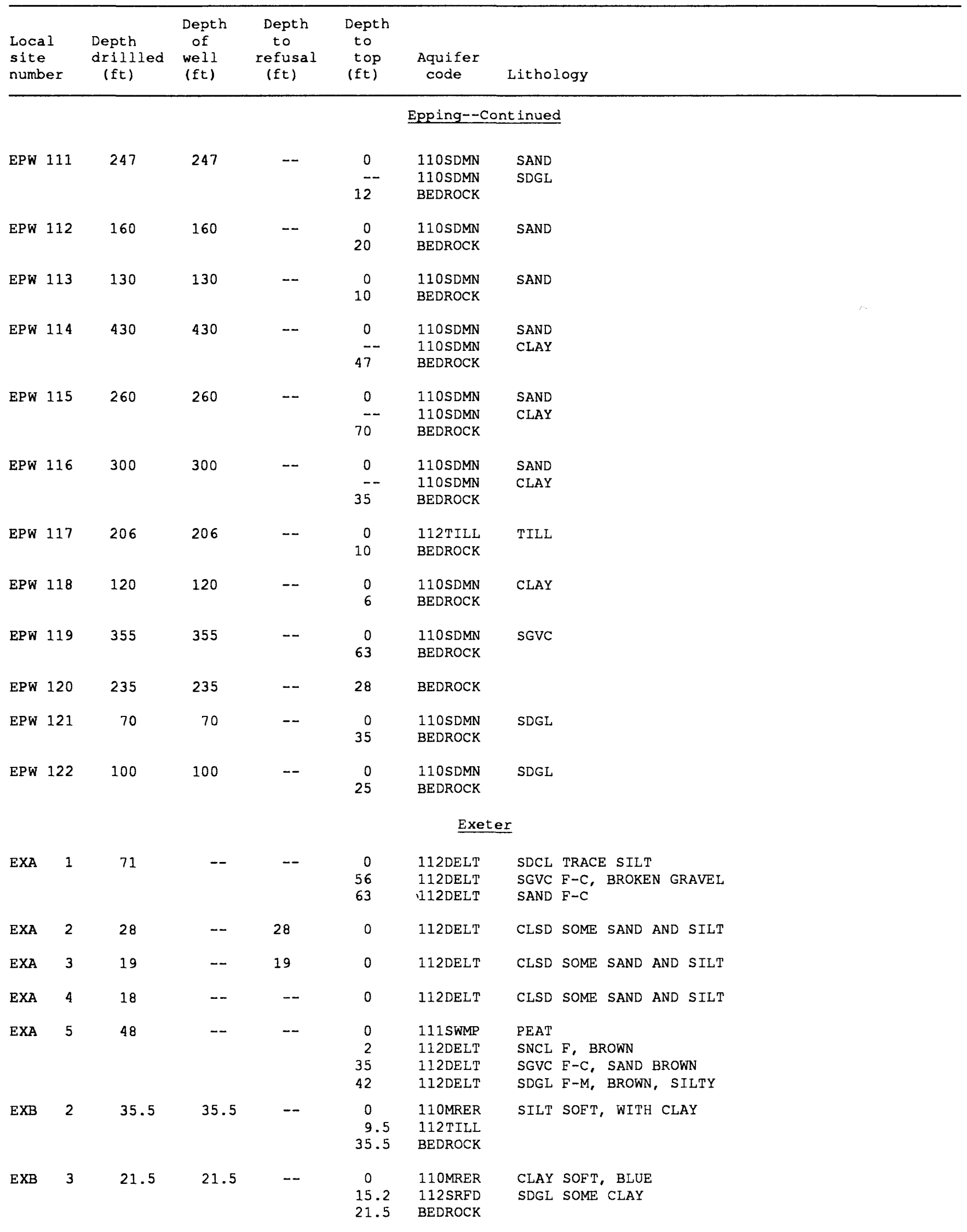


Table 3.--Lithologic logs of wells and borings--Continued

\begin{tabular}{|c|c|c|c|c|c|c|c|}
\hline \multicolumn{2}{|c|}{$\begin{array}{l}\text { Local } \\
\text { site } \\
\text { no }\end{array}$} & \multirow[t]{2}{*}{$\begin{array}{l}\text { Depth } \\
\text { drilliled } \\
\quad(\mathrm{ft})\end{array}$} & \multirow[t]{2}{*}{$\begin{array}{l}\text { Depth } \\
\text { of } \\
\text { well } \\
(f t)\end{array}$} & \multirow[t]{2}{*}{$\begin{array}{l}\text { Depth } \\
\text { to } \\
\text { refusal } \\
\text { (ft) }\end{array}$} & \multirow[t]{2}{*}{$\begin{array}{l}\text { Depth } \\
\text { to } \\
\text { top } \\
\text { (ft) }\end{array}$} & $\begin{array}{l}\text { Aquifer } \\
\text { code }\end{array}$ & \multirow[t]{2}{*}{ Lithology } \\
\hline & & & & & & Exeter--C & \\
\hline EXB & 4 & 29 & 29 & -- & $\begin{array}{r}0 \\
1 \\
11 \\
16\end{array}$ & $\begin{array}{l}110 \text { SOIL } \\
112 \text { SRFD } \\
112 \text { SRFD } \\
112 \text { TLL }\end{array}$ & $\begin{array}{l}\text { SAND } \mathrm{F} \\
\text { GRVL SILTY } \\
\text { TILL SANDY }\end{array}$ \\
\hline EXB & 6 & 19 & 19 & -- & $\begin{array}{r}0 \\
2 \\
8 \\
19\end{array}$ & $\begin{array}{l}\text { 111FILL } \\
112 \mathrm{MRIN} \\
112 \mathrm{TILL} \\
\text { BEDROCK }\end{array}$ & $\begin{array}{l}\text { GRVL } \\
\text { SILT WITH CLAY }\end{array}$ \\
\hline EXS & 1 & - & -- & -- & 0 & 112SRFD & SAND OVER MARINE DEPOSITS \\
\hline EXW & 2 & 56 & 56 & -- & $\begin{array}{r}0 \\
10 \\
47 \\
48\end{array}$ & $\begin{array}{l}112 \mathrm{MRIN} \\
112 \mathrm{MRIN} \\
112 \mathrm{SRFD} \\
112 \text { SRFD }\end{array}$ & $\begin{array}{l}\text { SDCL }-- \\
\text { CLAY GRAY AND BLUE } \\
\text { SAND F } \\
\text { SDGL }\end{array}$ \\
\hline EXW & 3 & -- & 16 & -- & 0 & 112SRFD & SAND \\
\hline EXW & 4 & -- & 29.3 & -- & 0 & $112 \mathrm{TILL}$ & TILL \\
\hline EXW & 5 & 170 & 170 & - & $\begin{array}{r}0 \\
100\end{array}$ & $\begin{array}{l}112 \text { TILL } \\
\text { BEDROCK }\end{array}$ & TILL \\
\hline EXW & 10 & 110 & 110 & -- & $\begin{array}{r}0 \\
85\end{array}$ & $\begin{array}{l}\text { 112SRFD } \\
\text { BEDROCK }\end{array}$ & SDCL -- \\
\hline EXW & 11 & 140 & 140 & -- & $\begin{array}{r}0 \\
90\end{array}$ & $\begin{array}{l}\text { 112SRFD } \\
\text { BEDROCK }\end{array}$ & SDGL MOSTLY F SAND \\
\hline EXW & 12 & -- & 12.3 & -- & 0 & 112 TILL & TILL \\
\hline EXW & 13 & -- & 52 & -- & 0 & 112SRFD & SDGL \\
\hline ExW & 14 & -- & 22 & -- & 0 & 112SRFD & SAND \\
\hline EXW & 15 & 62 & 60 & -- & $\begin{array}{r}5 \\
18 \\
58 \\
62\end{array}$ & $\begin{array}{l}\text { 112MRIN } \\
112 \text { SRFD } \\
\text { 112TILL } \\
\text { BEDROCK }\end{array}$ & $\begin{array}{ll}\text { CLAY HARD GRAY } \\
\text { SDGL INCLUDES COBBLES } \\
\text { TILL HARDPAN }\end{array}$ \\
\hline EXW & 17 & 86 & 86 & -- & $\begin{array}{r}0 \\
14 \\
80 \\
86\end{array}$ & $\begin{array}{l}\text { 112SRFD } \\
\text { 112MRIN } \\
112 \text { TILL } \\
\text { BEDROCK }\end{array}$ & $\begin{array}{l}\text { SAND M BROWN } \\
\text { STCL -- } \\
\text { TILL GRAVEL AND CLAY }\end{array}$ \\
\hline $\mathrm{EXW}$ & 18 & 52 & 52 & -- & $\begin{array}{r}5 \\
22\end{array}$ & $\begin{array}{l}112 \mathrm{MRIN} \\
112 \text { SRFD }\end{array}$ & $\begin{array}{l}\text { CLAY } \\
\text { SAND F-M }\end{array}$ \\
\hline
\end{tabular}


Table 3.--Lithologic logs of wells and borings--Continued

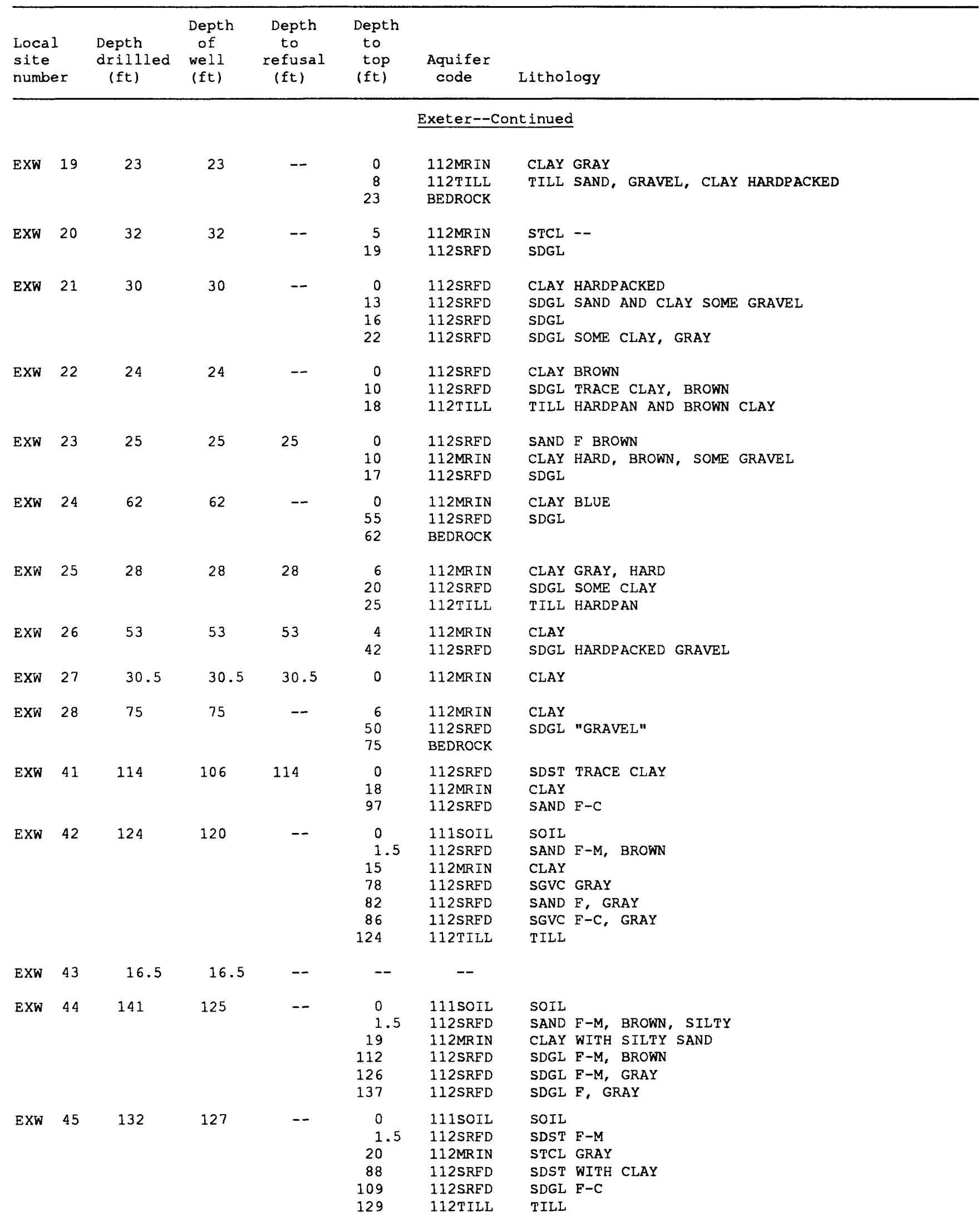


Table 3.--Lithologic logs of wells and borings--Continued

\begin{tabular}{|c|c|c|c|c|c|c|c|}
\hline \multicolumn{2}{|c|}{$\begin{array}{l}\text { Local } \\
\text { site } \\
\text { number }\end{array}$} & $\begin{array}{l}\text { Depth } \\
\text { drillled } \\
\text { (ft) }\end{array}$ & $\begin{array}{l}\text { Depth } \\
\text { of } \\
\text { well } \\
\text { (ft) }\end{array}$ & $\begin{array}{l}\text { Depth } \\
\text { to } \\
\text { refusal } \\
\text { (ft) }\end{array}$ & $\begin{array}{l}\text { Depth } \\
\text { to } \\
\text { top } \\
(f t)\end{array}$ & $\begin{array}{l}\text { Aquifer } \\
\text { code }\end{array}$ & Lithology \\
\hline & & & & \multicolumn{4}{|c|}{ Exeter--Continued } \\
\hline EXW & 46 & 92 & 89.5 & -- & $\begin{array}{l}0 \\
14.5 \\
75\end{array}$ & $\begin{array}{l}112 \text { SRFD } \\
112 \text { MRIN } \\
112 \text { SRFD }\end{array}$ & $\begin{array}{l}\text { SAND F-M, BROWN } \\
\text { CLAY WITH SILT } \\
\text { SDGL TRACE SILT AND CLAY }\end{array}$ \\
\hline EXW & 47 & 151 & 148 & -- & $\begin{array}{c}0 \\
1.5 \\
39 \\
117 \\
124\end{array}$ & $\begin{array}{l}111 \text { SOIL } \\
112 \text { SRFD } \\
112 \text { MRIN } \\
112 \text { SRFD } \\
112 \text { SRFD }\end{array}$ & $\begin{array}{l}\text { SOIL } \\
\text { SAND F, SILTY, GRAY } \\
\text { CLAY SILTY, SANDY } \\
\text { SNCL F } \\
\text { SDGL F-C }\end{array}$ \\
\hline EXW & 48 & 140 & 130 & 140 & $\begin{array}{r}0 \\
91 \\
105 \\
119\end{array}$ & $\begin{array}{l}112 \mathrm{MRIN} \\
112 \text { SRFD } \\
112 \text { SRFD } \\
112 \text { SRFD }\end{array}$ & $\begin{array}{l}\text { CLAY SANDY } 84-91 \text { FT } \\
\text { SAND F, CLAYEY } \\
\text { SDGL F } \\
\text { SDGL M-C, ANGULAR }\end{array}$ \\
\hline EXW & 49 & 127 & 127 & 127 & $\begin{array}{r}0 \\
18 \\
82 \\
115\end{array}$ & $\begin{array}{l}112 \text { SRFD } \\
112 \mathrm{MRIN} \\
112 \mathrm{SRFD} \\
112 \mathrm{SRFD}\end{array}$ & $\begin{array}{l}\text { SDST } F-M, \text { BROWN } \\
\text { STCL GRAY } \\
\text { SDST F-M, BROWN } \\
\text { SDGL F-C }\end{array}$ \\
\hline EXW & 50 & 17 & 16 & -- & -- & -- & \\
\hline EXW & 51 & 20.5 & 20.5 & 20.5 & $\begin{array}{l}0 \\
1 \\
8\end{array}$ & $\begin{array}{l}111 \text { SOIL } \\
112 \text { MRIN } \\
112 \text { SRED }\end{array}$ & $\begin{array}{l}\text { SOIL } \\
\text { CLSD BROWN } \\
\text { SGVC BROWN }\end{array}$ \\
\hline EXW & 52 & 9.5 & 9.5 & -- & 0 & 112 TLL & TILL \\
\hline EXW & 53 & 21 & 21 & -- & 21 & $112 \mathrm{GLCL}$ & OBDN \\
\hline EXW & 54 & 17.5 & 17.5 & -- & 0 & $112 \mathrm{TILL}$ & TILL \\
\hline EXW & 55 & 17 & 17 & -- & 0 & 112 TILL & TILL \\
\hline EXW & 56 & 38.5 & 32 & -- & $\begin{array}{r}0 \\
10\end{array}$ & $\begin{array}{l}\text { 111SOIL } \\
112 \text { SRFD }\end{array}$ & $\begin{array}{l}\text { SOIL } \\
\text { SDGL BROWN, WITH SILT }\end{array}$ \\
\hline EXW & 57 & 44 & 19 & -- & $\begin{array}{r}0 \\
1 \\
44\end{array}$ & $\begin{array}{l}111 \text { SOIL } \\
112 \text { SRFD } \\
112 \text { TILL }\end{array}$ & $\begin{array}{l}\text { SOIL } \\
\text { SDGL BROWN WITH GRAY SILT } \\
\text { TILL }\end{array}$ \\
\hline EXW & 58 & 123 & 119 & -- & $\begin{array}{r}0 \\
2 \\
19 \\
113 \\
121\end{array}$ & $\begin{array}{l}111 \text { SOIL } \\
112 \text { SRFD } \\
112 \text { MRIN } \\
112 \text { SRFD } \\
112 \text { TILL }\end{array}$ & $\begin{array}{l}\text { SOIL } \\
\text { SDST F-M, BROWN } \\
\text { STCL } \\
\text { SDGL F-M-C } \\
\text { TILL }\end{array}$ \\
\hline EXW & 59 & 97 & 97 & -- & $\begin{array}{r}0 \\
2 \\
29 \\
92 \\
96\end{array}$ & $\begin{array}{l}111 \text { SOIL } \\
112 \text { SRFD } \\
112 \mathrm{MRIN} \\
112 \text { SRFD } \\
112 \text { TILL }\end{array}$ & $\begin{array}{l}\text { SOIL } \\
\text { SDST WITH CLAY } \\
\text { CLAY SILTY } \\
\text { SDGL } \\
\text { TILL }\end{array}$ \\
\hline EXW & 60 & 49 & 30 & -- & $\begin{array}{r}0 \\
5 \\
22 \\
48\end{array}$ & $\begin{array}{l}111 \text { FILL } \\
112 \mathrm{MRIN} \\
112 \text { SRFD } \\
112 \text { SRFD }\end{array}$ & $\begin{array}{l}\text { CLAY GRAY } \\
\text { SDGL C-F } \\
\text { SAND F-M, GRAY }\end{array}$ \\
\hline EXW & 61 & 81 & 81 & 81 & 0 & $112 \mathrm{GLCL}$ & BLDR WITH SILT, BROWN \\
\hline EXW & 62 & 80 & 80 & -- & $\begin{array}{r}0 \\
22\end{array}$ & $\begin{array}{l}110 S D M N \\
\text { BEDROCK }\end{array}$ & SDGL \\
\hline
\end{tabular}


Table 3.--Lithologic logs of wells and borings--Continued

\begin{tabular}{|c|c|c|c|c|c|c|c|}
\hline \multicolumn{2}{|c|}{$\begin{array}{l}\text { Local } \\
\text { site } \\
\text { number }\end{array}$} & $\begin{array}{l}\text { Depth } \\
\text { drililed } \\
\quad(f t)\end{array}$ & $\begin{array}{l}\text { Depth } \\
\text { of } \\
\text { well } \\
\text { (ft) }\end{array}$ & $\begin{array}{l}\text { Depth } \\
\text { to } \\
\text { refusal } \\
\text { (ft) }\end{array}$ & $\begin{array}{l}\text { Depth } \\
\text { to } \\
\text { top } \\
\text { (ft) }\end{array}$ & $\begin{array}{l}\text { Aquifer } \\
\text { code }\end{array}$ & Lithology \\
\hline & & & \multicolumn{5}{|c|}{ Exeter--Cont inued } \\
\hline EXW & 63 & 62 & 62 & -- & $\begin{array}{r}0 \\
16\end{array}$ & $\begin{array}{l}\text { IIOSDMN } \\
\text { BEDROCK }\end{array}$ & SDGL \\
\hline EXW & 64 & 225 & 225 & -- & $\begin{array}{r}0 \\
32\end{array}$ & $\begin{array}{l}110 S D M N \\
\text { BEDROCK }\end{array}$ & SDGL \\
\hline EXW & 65 & 142 & 142 & -- & $\begin{array}{r}0 \\
29\end{array}$ & $\begin{array}{l}11 O S D M N \\
\text { BEDROCK }\end{array}$ & SDGL \\
\hline EXW & 66 & 60 & 60 & - & $\frac{0}{14}$ & $\begin{array}{l}110 S D M N \\
110 S D M N \\
\text { BEDROCK }\end{array}$ & $\begin{array}{l}\text { SAND } \\
\text { CLAY }\end{array}$ \\
\hline EXW & 67 & 135 & 135 & -- & $\begin{array}{r}0 \\
15\end{array}$ & $\begin{array}{l}110 \text { SDMN } \\
\text { BEDROCK }\end{array}$ & SDGL \\
\hline EXW & 68 & 297 & 297 & -- & $\begin{array}{c}0 \\
-- \\
-- \\
6\end{array}$ & $\begin{array}{l}110 S D M N \\
110 S D M N \\
110 S D M N \\
110 S D M N \\
\text { BEDROCK }\end{array}$ & $\begin{array}{l}\text { SAND } \\
\text { SDGL } \\
\text { CLAY } \\
\text { SDGL }\end{array}$ \\
\hline EXW & 69 & 320 & 320 & -- & 5 & BEDROCK & \\
\hline $\mathrm{EXW}$ & 70 & 125 & 125 & -- & 14 & BEDROCK & \\
\hline EXW & 71 & 220 & 220 & - & $\begin{array}{r}0 \\
38\end{array}$ & $\begin{array}{l}110 \text { SDMN } \\
\text { BEDROCK }\end{array}$ & CLAY \\
\hline EXW & 72 & 220 & 220 & -- & $\begin{array}{l}0 \\
9\end{array}$ & $\begin{array}{l}112 \text { TILL } \\
\text { BEDROCK }\end{array}$ & TILL \\
\hline EXW & 73 & 180 & 180 & -- & $\begin{array}{r}0 \\
35\end{array}$ & $\begin{array}{l}110 \text { SDMN } \\
\text { BEDROCK }\end{array}$ & CLAY \\
\hline EXW & 74 & 575 & 575 & -- & $\begin{array}{l}0 \\
8\end{array}$ & $\begin{array}{l}110 S D M N \\
\text { BEDROCK }\end{array}$ & SAND \\
\hline EXW & 75 & 350 & 350 & - & $\begin{array}{l}0 \\
8\end{array}$ & $\begin{array}{l}110 S D M N \\
\text { BEDROCK }\end{array}$ & SAND \\
\hline EXW & 76 & 300 & 300 & -- & 0 & $110 \mathrm{SDMN}$ & SAND \\
\hline & & & \multicolumn{5}{|c|}{ Fremont } \\
\hline FMA & 1 & 19 & -- & 17 & $\begin{array}{r}0 \\
13 \\
17 \\
18\end{array}$ & $\begin{array}{l}112 \text { SRFD } \\
112 \text { TILL } \\
112 \text { TILL } \\
\text { BEDROCK }\end{array}$ & $\begin{array}{l}\text { SAND M-C WITH PEBBLES } \\
\text { TILL } \\
\text { TILL } \\
\text { OTHR ROTTEN ROCK }\end{array}$ \\
\hline FMA & 2 & 13 & - & 13 & $\begin{array}{c}0 \\
11.5\end{array}$ & $\begin{array}{l}\text { 112SRFD } \\
112 \mathrm{TILL}\end{array}$ & $\begin{array}{l}\text { SDGL WITH PEBBLES AND COBBLES } \\
\text { TILL HARD }\end{array}$ \\
\hline FMA & 3 & 22 & -- & 22 & $\begin{array}{r}0 \\
16 \\
20\end{array}$ & $\begin{array}{l}112 \text { SRFD } \\
112 \text { TILL } \\
\text { BEDROCK }\end{array}$ & $\begin{array}{l}\text { SDGL F-VC SAND WITH COBBLES } \\
\text { TILL SANDY, WEATHERED ROCK }\end{array}$ \\
\hline FMW & 1 & 14.7 & 14.7 & -- & 0 & 112 TILL & \\
\hline FMW & 2 & 50 & 50 & -- & 3 & BEDROCK & MMPC -- \\
\hline FMW & 3 & 29 & 27 & 29 & $\begin{array}{r}0 \\
28\end{array}$ & $\begin{array}{l}112 \text { SRFD } \\
112 \mathrm{TILL}\end{array}$ & $\begin{array}{l}\text { SDGL VF-C SAND, PEBBLES } \\
\text { TILL }\end{array}$ \\
\hline
\end{tabular}


Table 3.--Lithologic logs of wells and borings--Continued

\begin{tabular}{|c|c|c|c|c|c|c|c|}
\hline \multicolumn{2}{|c|}{$\begin{array}{l}\text { Local } \\
\text { site } \\
\text { number }\end{array}$} & $\begin{array}{l}\text { Depth } \\
\text { drillled } \\
\text { (ft) }\end{array}$ & $\begin{array}{l}\text { Depth } \\
\text { of } \\
\text { well } \\
\text { (ft) }\end{array}$ & $\begin{array}{l}\text { Depth } \\
\text { to } \\
\text { refusal } \\
\text { (ft) }\end{array}$ & $\begin{array}{l}\text { Depth } \\
\text { to } \\
\text { top } \\
\text { (ft) }\end{array}$ & $\begin{array}{l}\text { Aquifer } \\
\text { code }\end{array}$ & Lithology \\
\hline & & & & \multicolumn{4}{|c|}{ Fremont--Continued } \\
\hline FMW & 4 & 24.5 & 16.6 & -- & $\begin{array}{l}0 \\
5 \\
16 \\
17.5 \\
20 \\
24\end{array}$ & $\begin{array}{l}112 \text { SRFD } \\
112 \text { SRFD } \\
112 \text { SRFD } \\
112 \text { SRFD } \\
112 \text { TILL } \\
\text { BEDROCK }\end{array}$ & $\begin{array}{l}\text { SAND F LIGHT BROWN } \\
\text { SAND M, RED } \\
\text { SAND C } \\
\text { SDGL } \\
\text { TILL } \\
\text { BRCC ROTTEN ROCK }\end{array}$ \\
\hline FMW & 5 & 17 & 13.5 & -- & $\begin{array}{r}3 \\
13\end{array}$ & $\begin{array}{l}\text { 112SRFD } \\
\text { 112TILL }\end{array}$ & $\begin{array}{l}\text { SDGL F-C SAND, GRANULS } \\
\text { TILL }\end{array}$ \\
\hline FMW & 14 & -- & 22 & -- & -- & -- & \\
\hline FMW & 15 & -- & 240 & -- & -- & BEDROCK & \\
\hline EMW & 18 & -- & 7.6 & -- & -- & -- & \\
\hline FMW & 20 & -- & 250 & -- & -- & BEDROCK & \\
\hline FMW & 22 & -- & 14 & -- & -- & -- & \\
\hline FMW & 23 & -- & 65 & -- & -- & -- & \\
\hline FMW & 24 & -- & 18 & -- & -- & -- & \\
\hline FMW & 26 & -- & 116 & -- & -- & BEDROCK & \\
\hline FMW & 27 & -- & 14 & -- & -- & -- & \\
\hline FMW & 29 & -- & 200 & -- & -- & BEDROCK & \\
\hline EMW & 30 & -- & 50 & -- & -- & BEDROCK & \\
\hline FMW & 31 & -- & 24 & -- & -- & -- & \\
\hline FMW & 33 & -- & 150 & -- & -- & BEDROCK & \\
\hline FMW & 34 & -- & 14 & -- & -- & -- & \\
\hline FMW & 35 & -- & 145 & -- & -- & BEDROCK & \\
\hline FMW & 37 & -- & 20 & -- & -- & -- & \\
\hline FMW & 38 & 160 & 160 & -- & $\begin{array}{r}0 \\
15\end{array}$ & $\begin{array}{l}110 \text { SDMN } \\
\text { BEDROCK }\end{array}$ & SAND \\
\hline FMW & 39 & 280 & 280 & -- & $\begin{array}{r}0 \\
15\end{array}$ & $\begin{array}{l}110 \text { SDMN } \\
\text { BEDROCK }\end{array}$ & SAND \\
\hline FMW & 40 & 160 & 160 & -- & $\begin{array}{r}0 \\
18\end{array}$ & $\begin{array}{l}110 \text { SDMN } \\
\text { BEDROCK }\end{array}$ & SAND \\
\hline FMW & 41 & 180 & 180 & -- & $\begin{array}{r}0 \\
13\end{array}$ & $\begin{array}{l}110 \text { SDMN } \\
\text { BEDROCK }\end{array}$ & SDGL \\
\hline FMW & 42 & 120 & 120 & -- & $\begin{array}{r}0 \\
15\end{array}$ & $\begin{array}{l}110 S D M N \\
\text { BEDROCK }\end{array}$ & SAND \\
\hline FMW & 43 & 200 & 200 & -- & $\begin{array}{r}0 \\
25\end{array}$ & $\begin{array}{l}110 \text { SDMN } \\
\text { BEDROCK }\end{array}$ & SAND \\
\hline FMW & 44 & 200 & 200 & -- & $\begin{array}{r}0 \\
20\end{array}$ & $\begin{array}{l}110 \text { SDMN } \\
\text { BEDROCK }\end{array}$ & SAND \\
\hline FMW & 45 & 380 & 380 & -- & $\begin{array}{r}0 \\
15\end{array}$ & $\begin{array}{l}110 \text { SDMN } \\
\text { BEDROCK }\end{array}$ & SAND \\
\hline
\end{tabular}


Table 3.--Lithologic logs of wells and borings--Continued

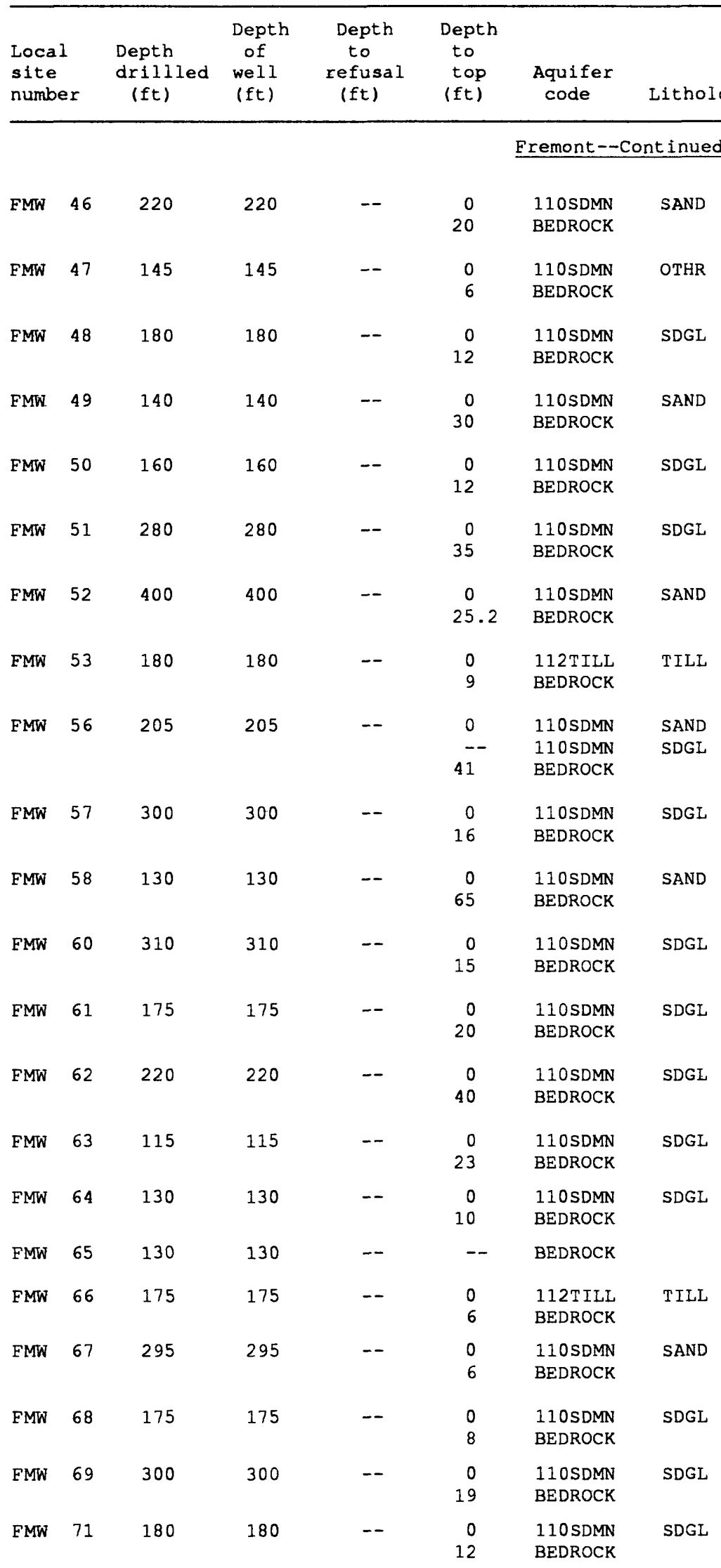


Table 3.--Lithologic logs of wells and borings--Continued

\begin{tabular}{|c|c|c|c|c|c|c|c|}
\hline \multicolumn{2}{|c|}{$\begin{array}{l}\text { Local } \\
\text { site } \\
\text { number }\end{array}$} & $\begin{array}{l}\text { Depth } \\
\text { drillled } \\
(\mathrm{ft})\end{array}$ & $\begin{array}{l}\text { Depth } \\
\text { of } \\
\text { well } \\
\text { (ft) }\end{array}$ & $\begin{array}{l}\text { Depth } \\
\text { to } \\
\text { refusal } \\
\text { (ft) }\end{array}$ & $\begin{array}{l}\text { Depth } \\
\text { to } \\
\text { top } \\
\text { (ft) }\end{array}$ & $\begin{array}{l}\text { Aquifer } \\
\text { code }\end{array}$ & Litholog \\
\hline & & & & & & \multicolumn{2}{|c|}{ Fremont--Cont inued } \\
\hline FMW & 72 & 124 & 124 & -- & $\begin{array}{r}0 \\
25\end{array}$ & $\begin{array}{l}110 \text { SDMN } \\
\text { BEDROCK }\end{array}$ & SDGL \\
\hline FMW & 73 & 205 & 205 & -- & 24 & BEDROCK & \\
\hline FMW & 74 & 245 & 245 & -- & $\begin{array}{r}0 \\
35\end{array}$ & $\begin{array}{l}\text { 112TILL } \\
\text { BEDROCK }\end{array}$ & TILL \\
\hline FMW & 75 & 315 & 315 & -- & $\begin{array}{c}0 \\
52\end{array}$ & $\begin{array}{l}112 \mathrm{TILL} \\
110 \mathrm{SDMN} \\
\text { BEDROCK }\end{array}$ & $\begin{array}{l}\text { TILL } \\
\text { CLAY }\end{array}$ \\
\hline FMW & 76 & 310 & 310 & -- & $\begin{array}{r}0 \\
10\end{array}$ & $\begin{array}{l}110 \text { SDMN } \\
\text { BEDROCK }\end{array}$ & SDGL \\
\hline FMW & 77 & 130 & 130 & -- & $\begin{array}{l}0 \\
8\end{array}$ & $\begin{array}{l}110 S D M N \\
\text { BEDROCK }\end{array}$ & SDGL \\
\hline & & & & & & Hamps & ead \\
\hline HDW & 2 & -- & 22.9 & -- & 0 & $112 \mathrm{TILI}$ & \\
\hline HDW & 29 & -- & 142 & -- & 40 & BEDROCK & ROCK -- \\
\hline HDW & 30 & -- & 163 & -- & 75 & BEDROCK & ROCK -- \\
\hline HDW & 31 & 280 & 280 & -- & $\begin{array}{r}0 \\
27\end{array}$ & $\begin{array}{l}112 \text { TLI } \\
\text { BEDROCK }\end{array}$ & TILL \\
\hline HDW & 32 & 150 & 150 & -- & 14 & BEDROCK & \\
\hline HDW & 33 & 145 & 145 & -- & $\begin{array}{r}0 \\
35\end{array}$ & $\begin{array}{l}110 S D M N \\
\text { BEDROCK }\end{array}$ & CLAY \\
\hline HDW & 34 & 105 & 105 & -- & $\begin{array}{r}0 \\
20\end{array}$ & $\begin{array}{l}110 \text { SDMN } \\
\text { BEDROCK }\end{array}$ & SDGL \\
\hline HDW & 35 & 200 & 200 & -- & $\begin{array}{r}0 \\
40\end{array}$ & $\begin{array}{l}110 S D M N \\
\text { BEDROCK }\end{array}$ & OTHR \\
\hline HDW & 36 & 150 & 150 & -- & $\begin{array}{r}0 \\
40\end{array}$ & $\begin{array}{l}110 \text { SDMN } \\
\text { BEDROCK }\end{array}$ & OTHR \\
\hline HDW & 37 & 150 & 150 & -- & 40 & BEDROCK & \\
\hline HDW & 38 & 620 & 620 & -- & 20 & BEDROCK & \\
\hline HDW & 39 & 300 & 300 & -- & $\begin{array}{r}0 \\
40\end{array}$ & $\begin{array}{l}110 S D M N \\
\text { BEDROCK }\end{array}$ & SDGL \\
\hline HDW & 40 & 220 & 220 & -- & $\begin{array}{r}0 \\
80\end{array}$ & $\begin{array}{l}112 \text { TILL } \\
\text { BEDROCK }\end{array}$ & TILL \\
\hline HDW & 41 & 235 & 235 & -- & $\begin{array}{r}0 \\
45\end{array}$ & $\begin{array}{l}112 \text { TILL } \\
\text { BEDROCK }\end{array}$ & TILL \\
\hline HDW & 42 & 280 & 280 & -- & $\begin{array}{r}0 \\
10\end{array}$ & $\begin{array}{l}112 \text { T ILL } \\
\text { BEDROCK }\end{array}$ & TILL \\
\hline HDW & 43 & 300 & 300 & -- & 16 & BEDROCK & \\
\hline
\end{tabular}


Table 3.--Lithologic logs of wells and borings--Continued

\begin{tabular}{|c|c|c|c|c|c|c|c|}
\hline $\begin{array}{l}\text { Loca } \\
\text { site } \\
\text { numbe }\end{array}$ & & $\begin{array}{l}\text { Depth } \\
\text { drililed } \\
\quad(f t)\end{array}$ & $\begin{array}{l}\text { Depth } \\
\text { of } \\
\text { wel1 } \\
(f t)\end{array}$ & $\begin{array}{l}\text { Depth } \\
\text { to } \\
\text { refusal } \\
(\mathrm{ft})\end{array}$ & $\begin{array}{l}\text { Depth } \\
\text { to } \\
\text { top } \\
(\mathrm{ft})\end{array}$ & $\begin{array}{l}\text { Aquifer } \\
\text { code }\end{array}$ & Lithology \\
\hline \multicolumn{8}{|c|}{ Hampstead--Continued } \\
\hline HDW : & 115 & 225 & 225 & -- & $\begin{array}{r}0 \\
85\end{array}$ & $\begin{array}{l}\text { 112TILL } \\
\text { BEDROCK }\end{array}$ & TILL \\
\hline HDW : & 116 & 475 & 475 & -- & $\frac{0}{38}$ & $\begin{array}{l}112 T I L L \\
110 S D M N \\
\text { BEDROCK }\end{array}$ & $\begin{array}{l}\text { TILL } \\
\text { CLAY }\end{array}$ \\
\hline \multicolumn{8}{|c|}{ Hampton Falls } \\
\hline HFA & 10 & 31.8 & - & 31.8 & $\begin{array}{r}0 \\
5.5 \\
17.5 \\
23.5\end{array}$ & $\begin{array}{l}112 \text { SRFD } \\
112 \text { SRFD } \\
112 \text { SRFD } \\
112 \text { SRFD }\end{array}$ & $\begin{array}{l}\text { COSD SAND, FINE TO COARSE, AND COBBLES } \\
\text { SAND SAND, FINE TO COARSE } \\
\text { SDGL SAND, FINE TO MEDIUM, AND GRAVEL } \\
\text { SGVC SAND AND GRAVEL, FINE TO COARSE; SOME CLAY }\end{array}$ \\
\hline HFW & 9 & -- & 21 & -- & 0 & $112 \mathrm{TILL}$ & TILL \\
\hline HFW & 10 & 100 & 100 & 11 & $\begin{array}{r}0 \\
11\end{array}$ & $\begin{array}{l}112 \text { TILL } \\
\text { BEDROCK }\end{array}$ & TILL "GRAVEL" \\
\hline \multicolumn{8}{|c|}{ Kensington } \\
\hline KFS & 1 & -- & -- & -- & 0 & 112 SRFD & SAND UNDER CLAY \\
\hline KFW & 5 & 120 & 120 & -- & $\begin{array}{r}0 \\
95\end{array}$ & $\begin{array}{l}112 \mathrm{TILL} \\
\text { BEDROCK }\end{array}$ & TILL \\
\hline KFW & 6 & 220 & 220 & -- & $\begin{array}{r}0 \\
200\end{array}$ & $\begin{array}{l}112 \text { TILL } \\
\text { BEDROCK }\end{array}$ & TILL \\
\hline KFW & 7 & 50 & 50 & -- & $\begin{array}{r}0 \\
38 \\
48\end{array}$ & $\begin{array}{l}\text { 112SRFD } \\
112 \text { SRFD } \\
\text { BEDROCK }\end{array}$ & $\begin{array}{l}\text { SAND F, ROCKY, AND SILT } \\
\text { SDGL F } \rightarrow C \text { SAND, C GRAVEL }\end{array}$ \\
\hline $\mathrm{KFW}$ & 8 & 60 & 60 & -- & $\begin{array}{r}0 \\
40\end{array}$ & $\begin{array}{l}112 \text { SRFD } \\
\text { BEDROCK }\end{array}$ & SDGL "GRAVEL" \\
\hline KFW & 9 & -- & 25 & -- & 0 & $112 \mathrm{TILL}$ & TILL \\
\hline \multicolumn{8}{|c|}{ Kingston } \\
\hline TA & 16 & 49 & -- & -- & $\begin{array}{r}3 \\
7 \\
20\end{array}$ & $\begin{array}{l}112 \text { SRFD } \\
112 \text { MRIN } \\
112 \text { MRIN }\end{array}$ & $\begin{array}{l}\text { SDST F SAND AND SILT } \\
\text { STCL -- } \\
\text { STCL WITH SAND AT } 41 \text { AND } 46 \text { FT }\end{array}$ \\
\hline
\end{tabular}


Table 3.--Lithologic logs of wells and borings--Continued

\begin{tabular}{|c|c|c|c|c|c|c|c|}
\hline \multicolumn{2}{|c|}{$\begin{array}{l}\text { Local } \\
\text { site } \\
\text { number }\end{array}$} & \multirow{3}{*}{$\begin{array}{l}\text { Depth } \\
\text { drillied } \\
(f t) \\
\\
23\end{array}$} & \multirow{3}{*}{$\begin{array}{l}\begin{array}{c}\text { Depth } \\
\text { of } \\
\text { well } \\
\text { (ft) }\end{array} \\
\\
\end{array}$} & \multirow{3}{*}{$\begin{array}{l}\begin{array}{l}\text { Depth } \\
\text { to } \\
\text { refusal } \\
\text { (ft) }\end{array} \\
\\
--\end{array}$} & \multirow[t]{2}{*}{$\begin{array}{l}\text { Depth } \\
\text { to } \\
\text { top } \\
\text { (ft) }\end{array}$} & $\begin{array}{l}\text { Aquifer } \\
\text { code }\end{array}$ & \multirow[t]{2}{*}{ Lithology } \\
\hline & & & & & & ingston-- & \\
\hline KTA & 17 & & & & $\begin{array}{l}0 \\
6 \\
15 \\
16 \\
19 \\
20 \\
21.5\end{array}$ & $\begin{array}{l}112 \text { SRED } \\
112 \text { SRFD } \\
112 \text { SRFD } \\
112 \text { SRFD } \\
112 \text { MRIN } \\
112 \text { SRFD } \\
112 \text { MRIN }\end{array}$ & $\begin{array}{l}\text { SDGL VFS TO TR PB }(1.5 \mathrm{~mm}), \text { MST FS } \\
\text { SDGL VFS TO PB (1mm), MST VES } \\
\text { SAND VF-F S, MST FS } \\
\text { SDGL FS TO PB (1mm), MST CS } \\
\text { SDST SILT-VC SAND, GRAY } \\
\text { SAND VE-VC, MSTLY FS } \\
\text { SDST SILT-VC SAND, GRAY }\end{array}$ \\
\hline KTA & 18 & 24 & -- & -- & $\begin{array}{r}0 \\
11 \\
14\end{array}$ & $\begin{array}{l}112 \text { SRFD } \\
112 \text { MRIN } \\
112 \text { MRIN }\end{array}$ & $\begin{array}{l}\text { SAND F-VF, SILT AT } 7-7.5 \\
\text { SILT SOME SAND } \\
\text { STCL -- }\end{array}$ \\
\hline KTA & 19 & 55 & -- & 55 & $\begin{array}{l}0 \\
23.7 \\
40 \\
52\end{array}$ & $\begin{array}{l}112 \text { NRSR } \\
112 \text { MRIN } \\
112 \text { MIN } \\
112 \text { TLI }\end{array}$ & $\begin{array}{l}\text { SAND FINE TO VERY FINE } \\
\text { SNCL LAM, F-VF SAND } \\
\text { SDCL F-VF SAND, SILT, CLAY } \\
\text { TILL CLAY MATRIX }\end{array}$ \\
\hline KTW & 1 & -- & 13 & -- & 0 & 112SRFD & SAND -- \\
\hline $\mathrm{KTW}$ & 2 & -- & 14 & -- & 0 & 112TILI & TILL \\
\hline KTW & 4 & 164 & 164 & -- & $\begin{array}{r}0 \\
155\end{array}$ & $\begin{array}{l}\text { 112TILL } \\
\text { BEDROCK }\end{array}$ & TILL \\
\hline KTW & 11 & -- & 13 & -- & 0 & 112SRFD & SAND -- \\
\hline KTW & 13 & -- & 12.5 & -- & 0 & $112 \mathrm{SRED}$ & SDGL -- \\
\hline KTW & 19 & 84 & 55 & -- & $\begin{array}{r}0 \\
22 \\
43 \\
53 \\
70 \\
80\end{array}$ & $\begin{array}{l}112 \text { SRFD } \\
112 \text { SRFD } \\
112 \text { SRFD } \\
112 \text { SRFD } \\
112 \text { SRFD } \\
112 \text { TILL }\end{array}$ & $\begin{array}{l}\text { SDGL M-VC SAND, GRANULES, PEBBLES } \\
\text { SAND VF-M, LAYER OF SDGL AT } 29 \text { FT } \\
\text { SNCL VF-M, LAYERS } \\
\text { SAND F-C, LAMINATED } \\
\text { SDST LAYERS, SOME CLAY } \\
\text { TILL VF-E SAND MATRIX }\end{array}$ \\
\hline KTW & 30 & 380 & 380 & -- & $\begin{array}{r}0 \\
16\end{array}$ & $\begin{array}{l}110 S D M N \\
\text { BEDROCK }\end{array}$ & SDGL \\
\hline KTW & 31 & 300 & 300 & -- & $\begin{array}{r}0 \\
20\end{array}$ & $\begin{array}{l}\text { 112TILL } \\
\text { BEDROCK }\end{array}$ & TILL \\
\hline KTพ & 32 & 240 & 240 & -- & $\begin{array}{r}0 \\
30\end{array}$ & $\begin{array}{l}\text { 112TILL } \\
\text { BEDROCK }\end{array}$ & TILL \\
\hline KTW & 33 & 480 & 480 & -- & $\begin{array}{r}0 \\
16\end{array}$ & $\begin{array}{l}110 \text { SDMN } \\
\text { BEDROCK }\end{array}$ & SDGL \\
\hline KTW & 34 & 423 & 423 & -- & $\begin{array}{r}0 \\
15\end{array}$ & $\begin{array}{l}110 S D M N \\
\text { BEDROCK }\end{array}$ & SAND \\
\hline KTW & 35 & 293 & 293 & -- & $\begin{array}{r}0 \\
16\end{array}$ & $\begin{array}{l}\text { 110SDMN } \\
\text { BEDROCK }\end{array}$ & SDGL \\
\hline KTW & 36 & 243 & 243 & -- & $\begin{array}{r}0 \\
37\end{array}$ & $\begin{array}{l}110 \text { SDMN } \\
\text { BEDROCK }\end{array}$ & CLAY \\
\hline KT\% & 37 & 220 & 220 & -- & $\begin{array}{r}0 \\
145\end{array}$ & $\begin{array}{l}\text { 112TILL } \\
\text { BEDROCK }\end{array}$ & TILL \\
\hline KTW & 38 & 205 & 205 & -- & $\frac{0}{90}$ & $\begin{array}{l}112 \text { TILL } \\
110 \text { SDMN } \\
\text { BEDROCK }\end{array}$ & $\begin{array}{l}\text { TILL } \\
\text { CLAY }\end{array}$ \\
\hline
\end{tabular}


Table 3.--Lithologic logs of wells and borings--Continued

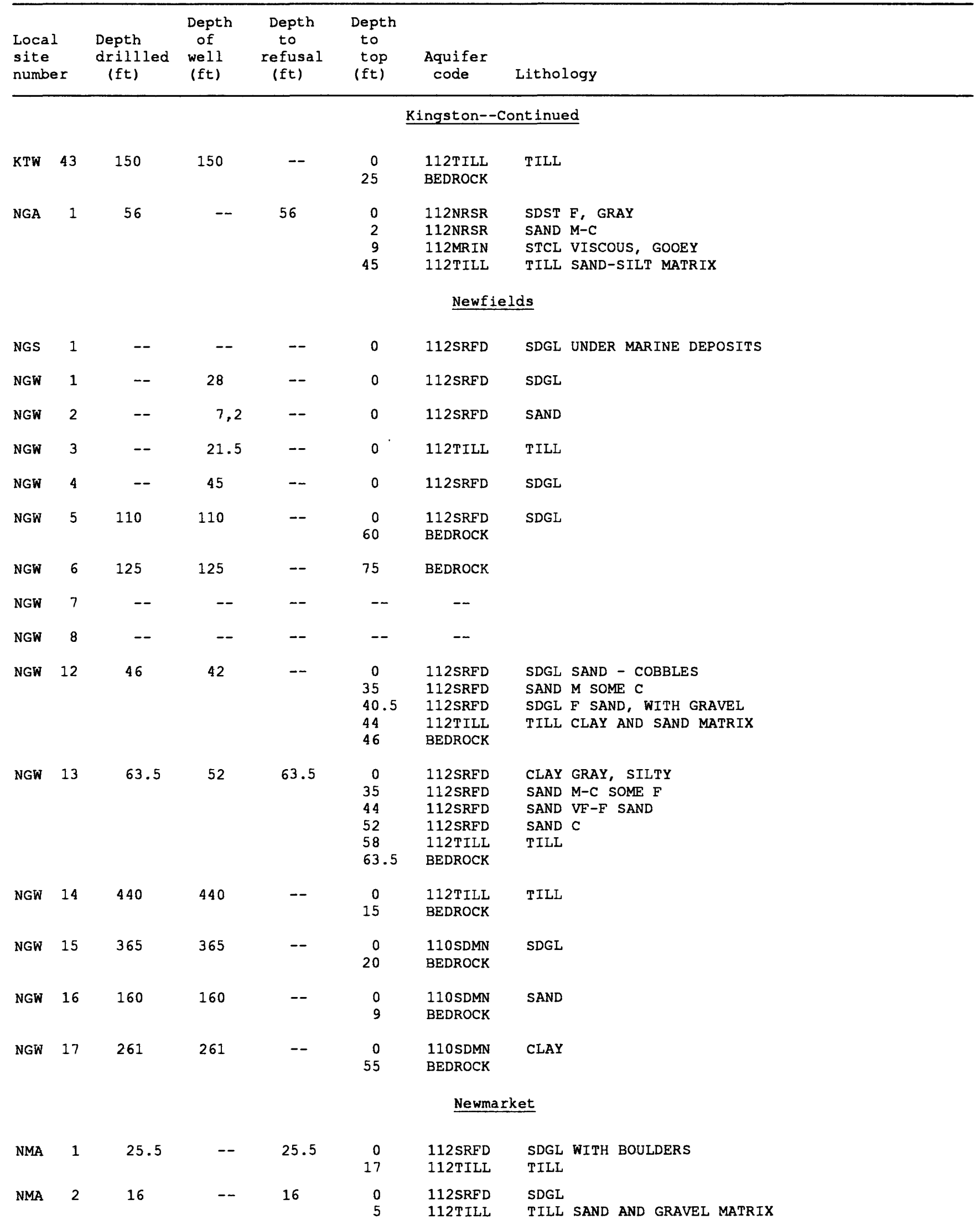


Table 3.--Lithologic logs of wells and borings--Continued

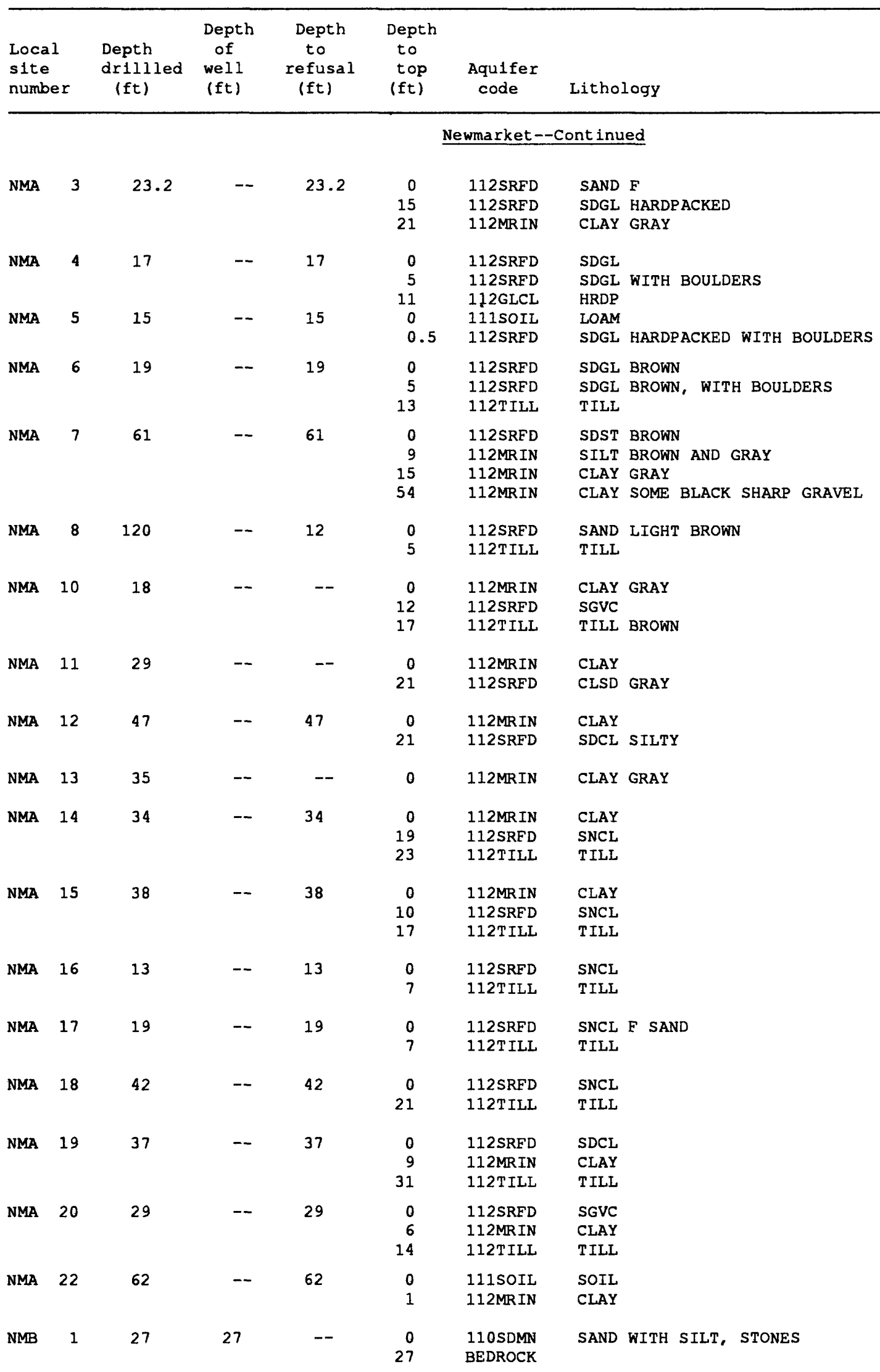


Table 3.--Lithologic logs of wells and borings--Continued

\begin{tabular}{|c|c|c|c|c|c|c|c|}
\hline \multicolumn{2}{|c|}{$\begin{array}{l}\text { Local } \\
\text { site } \\
\text { number }\end{array}$} & $\begin{array}{l}\text { Depth } \\
\text { drillled } \\
\text { (ft) }\end{array}$ & $\begin{array}{l}\text { Depth } \\
\text { of } \\
\text { well } \\
\text { (ft) }\end{array}$ & $\begin{array}{l}\text { Depth } \\
\text { to } \\
\text { refusal } \\
\text { (ft) }\end{array}$ & $\begin{array}{l}\text { Depth } \\
\text { to } \\
\text { top } \\
(f t)\end{array}$ & $\begin{array}{l}\text { Aquifer } \\
\text { code }\end{array}$ & Lithology \\
\hline & & & \multicolumn{5}{|c|}{ Newmarket--Cont Inued } \\
\hline NMS & 1 & -- & -- & -- & 0 & 112SRFD & SDGL OVER MARINE DEPOSITS \\
\hline NMS & 2 & -- & -- & -- & - & -- & \\
\hline NMS & 3 & -- & - & -- & 0 & 112SRFD & SAND UNDER CLAY \\
\hline NMW & 1 & -- & 11.7 & -- & 0 & 112SRFD & SAND \\
\hline NMW & 2 & -- & 57 & -- & 0 & 112SRFD & SAND \\
\hline NMW & 3 & -- & 20 & -- & 0 & 112SRFD & SDGL \\
\hline NMW & 4 & 238 & 238 & -- & $\begin{array}{r}0 \\
30 \\
38\end{array}$ & $\begin{array}{l}\text { 112MRIN } \\
112 \text { ILL } \\
\text { BEDROCK }\end{array}$ & $\begin{array}{l}\text { CLAY } \\
\text { TILL SAND AND ROCKS }\end{array}$ \\
\hline NMW & 6 & -- & 48 & - & 0 & 112DELT & SDGL \\
\hline NMW & 9 & 54 & 24.6 & -- & $\begin{array}{r}2 \\
10 \\
15 \\
23 \\
25 \\
54\end{array}$ & $\begin{array}{l}\text { 112SRFD } \\
112 \text { SRFD } \\
112 \text { SRFD } \\
112 \text { SRFD } \\
112 \text { SRFD } \\
\text { BEDROCK }\end{array}$ & $\begin{array}{l}\text { SILT WITH SOME GRANULES AND PEBBLES } \\
\text { SDGL C SAND, GRANULES } \\
\text { SAND VF-M SAND } \\
\text { SDGL C SAND, PEBBLES } \\
\text { SDST VF SAND, SILT AND CLAY, LAYERS }\end{array}$ \\
\hline NMW & 10 & 59.5 & 52.2 & -- & $\begin{array}{r}0 \\
35 \\
40 \\
50 \\
57\end{array}$ & $\begin{array}{l}112 \text { MRIN } \\
112 \text { MRIN } \\
112 \text { SRFD } \\
112 \text { MRIN } \\
112 \text { TILL }\end{array}$ & $\begin{array}{l}\text { CLAY SOME SILT AND SAND } \\
\text { SDST VF-F SAND, SILT LAYER } \\
\text { SAND F SOME M, TRACE SILT } \\
\text { STCL F-M SAND, ALTERNATING LAYERS } \\
\text { TILL }\end{array}$ \\
\hline NMW & 11 & 70 & 54 & -- & $\begin{array}{r}0 \\
20 \\
49 \\
55\end{array}$ & $\begin{array}{l}112 \mathrm{DELT} \\
112 \mathrm{DELT} \\
112 \mathrm{DELT} \\
112 \mathrm{TILL}\end{array}$ & $\begin{array}{l}\text { SAND VF-F OVER F-M } \\
\text { SAND F-C, STRKS SILT } \\
\text { GRVL PEBBLES, GRANULES, M-VC SAND } \\
\text { TILL SANDY, SILTY, PEBBLEY }\end{array}$ \\
\hline NMW & 12 & 75.5 & 75.5 & 76.5 & $\begin{array}{l}49 \\
61 \\
62\end{array}$ & $\begin{array}{l}112 \text { SRFD } \\
112 \text { SRFD } \\
112 \text { SRFD }\end{array}$ & $\begin{array}{l}\text { SAND F-C SAND, TRACE SILT } \\
\text { SAND F-C, REDISH BROWN } \\
\text { SDGL }\end{array}$ \\
\hline NMW & 13 & 140 & 140 & -- & $\begin{array}{r}0 \\
12\end{array}$ & $\begin{array}{l}\text { 110SDMN } \\
\text { BEDROCK }\end{array}$ & SDGL \\
\hline NMW & 14 & 100 & 100 & -- & $\begin{array}{r}0 \\
12\end{array}$ & $\begin{array}{l}\text { 110SDMN } \\
\text { BEDROCK }\end{array}$ & SAND \\
\hline NMW & 15 & 120 & 120 & -- & $\begin{array}{r}0 \\
10\end{array}$ & $\begin{array}{l}\text { 110SDMN } \\
\text { BEDROCK }\end{array}$ & SAND \\
\hline NMW & 16 & 200 & 200 & -- & $\begin{array}{r}0 \\
14\end{array}$ & $\begin{array}{l}\text { 11OSDMN } \\
\text { BEDROCK }\end{array}$ & SDGL \\
\hline NMW & 17 & 140 & 140 & -- & $\begin{array}{l}0 \\
4\end{array}$ & $\begin{array}{l}\text { 110SDMN } \\
\text { BEDROCK }\end{array}$ & SDGL \\
\hline NMW & 18 & 260 & 260 & -- & $\begin{array}{l}0 \\
4\end{array}$ & $\begin{array}{l}\text { 110SDMN } \\
\text { BEDROCK }\end{array}$ & SAND \\
\hline NMW & 19 & 160 & 160 & -- & $\begin{array}{l}0 \\
6\end{array}$ & $\begin{array}{l}\text { 110SDMN } \\
\text { BEDROCK }\end{array}$ & SDGL \\
\hline NMW & 20 & 120 & 120 & -- & $\begin{array}{l}0 \\
8\end{array}$ & $\begin{array}{l}\text { 110SDMN } \\
\text { BEDROCK }\end{array}$ & SDGL \\
\hline NMW & 21 & 150 & .150 & -- & $\begin{array}{r}0 \\
31\end{array}$ & $\begin{array}{l}\text { 112TILL } \\
\text { BEDROCK }\end{array}$ & TILL \\
\hline
\end{tabular}


Table 3.--Lithologic logs of wells and borings--Continued

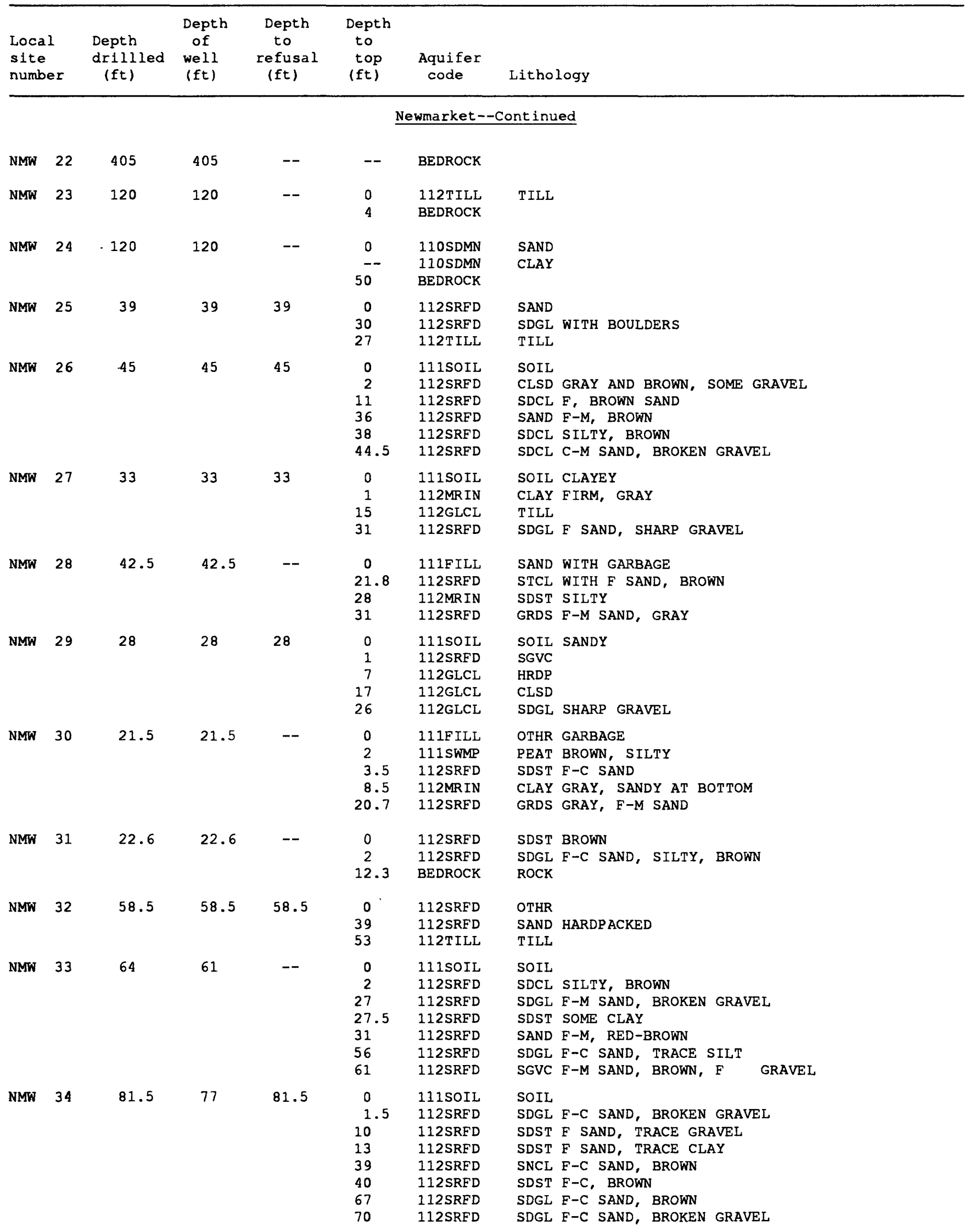


Table 3.--Lithologic logs of wells and borings--Continued

\begin{tabular}{|c|c|c|c|c|c|c|c|}
\hline \multicolumn{2}{|c|}{$\begin{array}{l}\text { Local } \\
\text { site } \\
\text { number }\end{array}$} & $\begin{array}{l}\text { Depth } \\
\text { drillled } \\
\quad(f t)\end{array}$ & $\begin{array}{l}\text { Depth } \\
\text { of } \\
\text { well } \\
(\mathrm{ft})\end{array}$ & $\begin{array}{l}\text { Depth } \\
\text { to } \\
\text { refusal } \\
\text { (ft) }\end{array}$ & $\begin{array}{l}\text { Depth } \\
\text { to } \\
\text { top } \\
(f t)\end{array}$ & $\begin{array}{l}\text { Aquifer } \\
\text { code }\end{array}$ & Lithology \\
\hline & & \multicolumn{6}{|c|}{ Newmarket--Cont inued } \\
\hline NMW & 35 & 22.6 & 22.6 & -- & $\begin{array}{l}0 \\
0.5 \\
4 \\
16.6\end{array}$ & $\begin{array}{l}\text { 111SOIL } \\
112 \text { MRIN } \\
112 \text { TILL } \\
\text { BEDROCK }\end{array}$ & $\begin{array}{l}\text { SOIL SILTY } \\
\text { STCL SANDY, BROWN, GRAY } \\
\text { TILL SILT, SAND, WITH COBBLES, GRAY } \\
\text { ROCK }\end{array}$ \\
\hline NMW & 36 & 31 & 31 & 31 & $\begin{array}{r}0 \\
1 \\
18 \\
27 \\
30\end{array}$ & $\begin{array}{l}\text { 111SOIL } \\
112 \text { SRFD } \\
112 \text { MRIN } \\
112 \text { SRFD } \\
112 T I L L\end{array}$ & $\begin{array}{l}\text { LOAM } \\
\text { SDCL F SAND } \\
\text { CLAY } \\
\text { SDCL C SAND } \\
\text { TILL }\end{array}$ \\
\hline NMW & 37 & 28.5 & 28.5 & 28.5 & $\begin{array}{r}0 \\
1 \\
7 \\
22\end{array}$ & $\begin{array}{l}111 \text { SOIL } \\
112 \text { SRFD } \\
112 \text { MRIN } \\
112 \text { SRFD }\end{array}$ & $\begin{array}{l}\text { LOAM } \\
\text { SDCL } \\
\text { CLAY } \\
\text { SDCL }\end{array}$ \\
\hline NMW & 38 & 34 & 34 & 34 & $\begin{array}{r}0 \\
1 \\
24 \\
32\end{array}$ & $\begin{array}{l}111 \text { SOIL } \\
112 \text { SRFD } \\
112 \text { MRIN } \\
112 \text { SRFD }\end{array}$ & $\begin{array}{l}\text { LOAM } \\
\text { SDCL } \\
\text { CLAY } \\
\text { SDCL }\end{array}$ \\
\hline NMW & 39 & 25.7 & 25.7 & -- & $\begin{array}{c}0 \\
2 \\
15.6\end{array}$ & $\begin{array}{l}111 \text { SOIL } \\
112 \text { MRIN } \\
112 \text { TILL }\end{array}$ & $\begin{array}{l}\text { SOIL SILTY, BROWN } \\
\text { STCL GRAY, WITH SAND LAYERS } \\
\text { TILL SAND, SILT, COBBLES }\end{array}$ \\
\hline NMW & 40 & 24.5 & 24.5 & -- & $\begin{array}{r}0 \\
1 \\
7 \\
14 \\
18\end{array}$ & $\begin{array}{l}111 \text { SOIL } \\
112 \text { SRFD } \\
112 \mathrm{GLCL} \\
112 \mathrm{MRIN} \\
112 \mathrm{TILL}\end{array}$ & $\begin{array}{l}\text { LOAM } \\
\text { CLAY WITH BOULDERS } \\
\text { TILL WITH BOULDERS } \\
\text { CLAY GRAY } \\
\text { TILL }\end{array}$ \\
\hline NMW & 41 & 49.5 & 49.5 & 49.5 & $\begin{array}{r}0 \\
2 \\
20 \\
25 \\
30 \\
40\end{array}$ & $\begin{array}{l}111 \text { FILI } \\
112 \text { SRFD } \\
112 \text { MRIN } \\
112 \text { SRFD } \\
112 \text { MRIN } \\
112 \text { SRFD }\end{array}$ & $\begin{array}{l}\text { GRVL } \\
\text { SDCL } \\
\text { CLAY BLUE } \\
\text { SDCL } \\
\text { CLAY } \\
\text { GRCL SHARP GRAVEL }\end{array}$ \\
\hline NMW & 42 & 59 & 59 & 59 & $\begin{array}{r}0 \\
1 \\
7 \\
31 \\
56\end{array}$ & $\begin{array}{l}111 \text { SOIL } \\
112 \text { SRFD } \\
112 \text { SRFD } \\
112 \text { MRIN } \\
112 \text { SRFD }\end{array}$ & $\begin{array}{l}\text { LOAM } \\
\text { SAND BROWN } \\
\text { SILT } \\
\text { STCL } \\
\text { GRCL SHARP GRAVEL, HARD CLAY }\end{array}$ \\
\hline NMW & 43 & 52 & 52 & 52 & $\begin{array}{l}0 \\
0.5 \\
9 \\
47\end{array}$ & $\begin{array}{l}111 \text { SOIL } \\
112 \text { SRFD } \\
112 \text { SRFD } \\
112 \text { SRFD }\end{array}$ & $\begin{array}{l}\text { LOAM } \\
\text { SAND F, BROWN } \\
\text { GRSC SHARP GRAVEL } \\
\text { SDGL BROWN SAND, SHARP GRAVEL }\end{array}$ \\
\hline NMW & 44 & 56 & 56 & 56 & $\begin{array}{r}0 \\
1 \\
6 \\
44\end{array}$ & $\begin{array}{l}111 \text { SOIL } \\
112 \text { SRFD } \\
112 \text { MRIN } \\
112 \text { SRFD }\end{array}$ & $\begin{array}{l}\text { LOAM } \\
\text { SAND F } \\
\text { CLAY SOFT GRAY } \\
\text { SNCL F, BROWN, SAND }\end{array}$ \\
\hline NMW & 45 & 113 & 113 & 113 & $\begin{array}{r}0 \\
17 \\
30 \\
103\end{array}$ & $\begin{array}{l}\text { 112SRFD } \\
112 \mathrm{MRIN} \\
112 \mathrm{MRIN} \\
112 \text { SRFD }\end{array}$ & $\begin{array}{l}\text { SDGL BROWN } \\
\text { STCL WITH MICA FLAKES } \\
\text { CLAY GRAY } \\
\text { GRCL M, ANGULAR GRAVEL }\end{array}$ \\
\hline
\end{tabular}


Table 3.--Lithologic logs of wells and borings--Continued

\begin{tabular}{|c|c|c|c|c|c|c|c|c|}
\hline \multicolumn{2}{|c|}{$\begin{array}{l}\text { Local } \\
\text { site } \\
\text { number }\end{array}$} & $\begin{array}{l}\text { Depth } \\
\text { drilliled } \\
\text { (ft) }\end{array}$ & $\begin{array}{l}\text { Depth } \\
\text { of } \\
\text { well } \\
\text { (ft) }\end{array}$ & $\begin{array}{l}\text { Depth } \\
\text { to } \\
\text { refusal } \\
\text { (ft) }\end{array}$ & $\begin{array}{l}\text { Depth } \\
\text { to } \\
\text { top } \\
(\mathrm{ft})\end{array}$ & $\begin{array}{l}\text { Aquifer } \\
\text { code }\end{array}$ & Lithology & \\
\hline & & \multicolumn{7}{|c|}{ Newmarket--Cont inued } \\
\hline NMW & 46 & 106 & 106 & 106 & $\begin{array}{r}0 \\
12 \\
23 \\
91\end{array}$ & $\begin{array}{l}\text { 112SRFD } \\
112 \text { MRIN } \\
112 \text { MRIN } \\
112 \text { SRFD }\end{array}$ & $\begin{array}{l}\text { SNCL SILTY, GRAY } \\
\text { STCL GRAY, MICA FLAKES } \\
\text { CLAY GRAY } \\
\text { CLAY SOME M, BLACK GRAVEL }\end{array}$ & \\
\hline NMW & 47 & 70 & 70 & 70 & $\begin{array}{r}0 \\
6 \\
11 \\
50\end{array}$ & $\begin{array}{l}112 \text { SRFD } \\
112 \mathrm{MRIN} \\
112 \mathrm{MRIN} \\
112 \text { SRFD }\end{array}$ & $\begin{array}{l}\text { SAND F, BROWN } \\
\text { SILT GRAY } \\
\text { CLAY GRAY } \\
\text { SDGL BROWN SAND, ANGULAR GRAVEL }\end{array}$ & \\
\hline NMW & 48 & 65 & 65 & 65 & $\begin{array}{r}0 \\
10 \\
28 \\
39\end{array}$ & $\begin{array}{l}112 \text { SRFD } \\
112 \text { SRFD } \\
112 \mathrm{MRIN} \\
112 \mathrm{TILL}\end{array}$ & $\begin{array}{l}\text { SDGL C SAND, BOULDERS } \\
\text { SDGL BROWN } \\
\text { STCL BROWN } \\
\text { TILL }\end{array}$ & \\
\hline NMW & 49 & 48 & 48 & 48 & $\begin{array}{r}0 \\
27\end{array}$ & $\begin{array}{l}112 \text { SRFD } \\
112 \text { TILL }\end{array}$ & $\begin{array}{l}\text { SDGL BROWN } \\
\text { TILI }\end{array}$ & \\
\hline NMW & 50 & 30.5 & 30.5 & -- & $\begin{array}{r}0 \\
4.5 \\
10.5\end{array}$ & $\begin{array}{l}111 \text { SOIL } \\
112 \text { SRFD } \\
112 \text { TILL }\end{array}$ & $\begin{array}{l}\text { SOIL SILT, BROWN, WITH WOOD } \\
\text { STCL GRAY, SANDY } \\
\text { TILL GRANULES, SILT, SAND, COBBLES, }\end{array}$ & GRAY \\
\hline NMW & 51 & 38.7 & 38.7 & 38.7 & $\begin{array}{l}0 \\
14 \\
29.9\end{array}$ & $\begin{array}{l}111 \mathrm{FILL} \\
112 \text { SRFD } \\
112 \mathrm{TILL}\end{array}$ & $\begin{array}{l}\text { OTHR GARBAGE } \\
\text { STCL SANDY } \\
\text { TILL F-M SAND, SILT, GRAVEL }\end{array}$ & \\
\hline NMW & 52 & 59 & 59 & 59 & $\begin{array}{r}0 \\
29\end{array}$ & $\begin{array}{l}\text { 112SRFD } \\
112 \text { TILL }\end{array}$ & $\begin{array}{l}\text { SDGL F SAND, C GRAVEL } \\
\text { TILL }\end{array}$ & \\
\hline NMW & 53 & 20.1 & 20.1 & 20.1 & $\begin{array}{r}0 \\
0.5 \\
2.5 \\
13.7\end{array}$ & $\begin{array}{l}111 \text { SOIL } \\
112 \text { SRFD } \\
112 \text { MRIN } \\
112 \text { TILL }\end{array}$ & $\begin{array}{l}\text { SOIL BROWN, SILTY } \\
\text { SAND F-M, TR SILT, BROWN } \\
\text { SDST SILT, F SAND } \\
\text { TILL SAND, SILT, COBBLES }\end{array}$ & \\
\hline NMW & 54 & 13.4 & 13.4 & 13.4 & $\begin{array}{l}0 \\
4 \\
10.8\end{array}$ & $\begin{array}{l}112 \text { SRFD } \\
112 \mathrm{MRIN} \\
112 \mathrm{TILL}\end{array}$ & $\begin{array}{l}\text { GRDS F-M SAND, BROWN } \\
\text { CLSD BROWN, SILT, F SAND LAYERS } \\
\text { TILL SAND, SILT, COBBLES, BROWN }\end{array}$ & \\
\hline NMW & 55 & 21 & 21 & 21 & $\begin{array}{l}0 \\
0.5\end{array}$ & $\begin{array}{l}111 \text { SOIL } \\
112 \text { TILL }\end{array}$ & $\begin{array}{l}\text { SOIL BROWN } \\
\text { TILL SILT, SAND, COBBLES, BROWN }\end{array}$ & \\
\hline NMW & 56 & 12.2 & 12.2 & -- & $\begin{array}{r}0 \\
1.3 \\
10.4\end{array}$ & $\begin{array}{l}111 \text { SOIL } \\
112 \text { TILL } \\
\text { BEDROCK }\end{array}$ & $\begin{array}{l}\text { SOIL BROWN, SILTY } \\
\text { TILL SILT, SAND, GRAVEL, BROWN } \\
\text { ROCK WEATHERED }\end{array}$ & \\
\hline NMW & 58 & 28 & 28 & -- & $\begin{array}{r}0 \\
14\end{array}$ & $\begin{array}{l}112 \mathrm{MRIN} \\
112 \mathrm{SRFD}\end{array}$ & $\begin{array}{l}\text { CLAY } \\
\text { SDGL M-C SAND, F GRAVEL }\end{array}$ & \\
\hline NMW & 59 & 28 & 28 & -- & $\begin{array}{r}0 \\
14\end{array}$ & $\begin{array}{l}\text { 112MRIN } \\
112 \text { SRFD }\end{array}$ & $\begin{array}{l}\text { CLAY } \\
\text { SDGL F-C SAND }\end{array}$ & \\
\hline NMW & 61 & 100 & 100 & -- & $\begin{array}{l}0 \\
8\end{array}$ & $\begin{array}{l}\text { 110SDMN } \\
\text { BEDROCK }\end{array}$ & SAND & \\
\hline NMW & 62 & 220 & 220 & - & $\begin{array}{l}0 \\
4.5\end{array}$ & $\begin{array}{l}\text { 110SDMN } \\
\text { BEDROCK }\end{array}$ & SDGL & \\
\hline NMW & 63 & 120 & 120 & -- & $\begin{array}{r}0 \\
25\end{array}$ & $\begin{array}{l}\text { 11OSDMN } \\
\text { BEDROCK }\end{array}$ & CLAY & \\
\hline NMW & 64 & 155 & 155 & -- & -- & BEDROCK & & \\
\hline NMW & 65 & 263 & 263 & -- & $\begin{array}{r}0 \\
45\end{array}$ & $\begin{array}{l}112 \text { TILL } \\
\text { BEDROCK }\end{array}$ & TILL & \\
\hline
\end{tabular}


Table 3.--Lithologic logs of wells and borings--Continued

\begin{tabular}{|c|c|c|c|c|c|c|c|}
\hline \multicolumn{2}{|c|}{$\begin{array}{l}\text { Local } \\
\text { site } \\
\text { number }\end{array}$} & $\begin{array}{l}\text { Depth } \\
\text { drillled } \\
\text { (ft) }\end{array}$ & $\begin{array}{l}\text { Depth } \\
\text { of } \\
\text { well } \\
\text { (ft) }\end{array}$ & $\begin{array}{l}\text { Depth } \\
\text { to } \\
\text { refusal } \\
\text { (ft) }\end{array}$ & $\begin{array}{l}\text { Depth } \\
\text { to } \\
\text { top } \\
\text { (ft) }\end{array}$ & $\begin{array}{l}\text { Aquifer } \\
\text { code }\end{array}$ & Lithology \\
\hline & & & & & \multicolumn{3}{|c|}{ Newmarket--Cont inued } \\
\hline NMW & 66 & 220 & 220 & -- & $\begin{array}{l}0 \\
4\end{array}$ & $\begin{array}{l}112 \text { TILL } \\
\text { BEDROCK }\end{array}$ & TILL \\
\hline NMW & 67 & 122 & 122 & -- & -- & BEDROCK & \\
\hline NMW & 69 & 50.5 & 50.5 & 50.5 & $\begin{array}{c}0 \\
2 \\
38.5\end{array}$ & $\begin{array}{l}\text { 111SOIL } \\
112 \text { MRIN } \\
\text { 112SRFD }\end{array}$ & $\begin{array}{l}\text { SOIL } \\
\text { CLAY } \\
\text { GRVL F, SHA }\end{array}$ \\
\hline & & & & & \multicolumn{3}{|c|}{ Northwood } \\
\hline NWW & 2 & 16 & 16 & -- & 0 & 112 ICCC & SDGL \\
\hline NWW & 4 & 110 & 110 & -- & $\begin{array}{r}0 \\
15\end{array}$ & $\begin{array}{l}\text { 110SDMN } \\
\text { BEDROCK }\end{array}$ & \\
\hline NWW & 5 & 16.5 & 16.5 & -- & 0 & Nottingham & ham \\
\hline NXB & 1 & 17 & 17 & -- & $\begin{array}{r}0 \\
15 \\
17\end{array}$ & $\begin{array}{l}\text { 112SRFD } \\
112 \text { TILL } \\
\text { BEDROCK }\end{array}$ & SDGL SILTY \\
\hline NXB & 2 & 10 & 10 & -- & $\begin{array}{r}0 \\
10\end{array}$ & $\begin{array}{l}112 \mathrm{TILL} \\
\text { BEDROCK }\end{array}$ & TILL SANDY \\
\hline NXS & 1 & -- & -- & -- & 0 & 112SRFD & \\
\hline NXS & 2 & -- & -- & -- & 0 & 112SRFD & \\
\hline NXW & 2 & 19.5 & 19.5 & -- & 0 & $112 \mathrm{TILL}$ & \\
\hline NXW & 3 & 300 & 300 & -- & $\begin{array}{r}0 \\
30\end{array}$ & $\begin{array}{l}\text { 110SDMN } \\
\text { BEDROCK }\end{array}$ & \\
\hline $\mathrm{NXW}$ & 4 & 15.5 & 15.5 & -- & 0 & 112TILL & \\
\hline NXW & 5 & -- & 15 & -- & 0 & 112TILL & \\
\hline$N X W$ & 6 & 8.8 & 8.8 & - & 0 & $112 \mathrm{TILL}$ & \\
\hline NXW & 7 & 25 & 25 & -- & 0 & 110SDMN & \\
\hline NXW & 8 & 110 & 110 & -- & $\begin{array}{r}0 \\
70\end{array}$ & $\begin{array}{l}\text { 112SRFD } \\
\text { BEDROCK }\end{array}$ & $\begin{array}{l}\text { SDGL } \\
\text { SCST }\end{array}$ \\
\hline NXW & 9 & 310 & 310 & -- & 3 & BEDROCK & \\
\hline NXW & 12 & 225 & 225 & -- & $\begin{array}{r}0 \\
158\end{array}$ & $\begin{array}{l}\text { 110SDMN } \\
\text { BEDROCK }\end{array}$ & SAND \\
\hline NXW & 13 & 365 & 365 & -- & $\frac{0}{18}$ & $\begin{array}{l}110 \text { SDMN } \\
\text { 110SDMN } \\
\text { BEDROCK }\end{array}$ & $\begin{array}{l}\text { SDGL } \\
\text { CLAY }\end{array}$ \\
\hline NXW & 14 & 102 & 102 & -- & $\begin{array}{l}0 \\
5\end{array}$ & $\begin{array}{l}\text { 110SDMN } \\
\text { BEDROCK }\end{array}$ & SDGL \\
\hline NXW & 15 & 235 & 235 & -- & 30 & BEDROCK & \\
\hline NXW & 16 & 145 & 145 & -- & $\begin{array}{l}0 \\
3\end{array}$ & $\begin{array}{l}\text { 112TILL } \\
\text { BEDROCK }\end{array}$ & TILL \\
\hline
\end{tabular}


Table 3.--Lithologic logs of wells and borings--Continued

\begin{tabular}{|c|c|c|c|c|c|c|c|}
\hline \multirow{2}{*}{\multicolumn{2}{|c|}{$\begin{array}{l}\text { Local } \\
\text { site }\end{array}$}} & \multirow[t]{2}{*}{$\begin{array}{l}\text { Depth } \\
\text { drillied } \\
\quad \text { (ft) }\end{array}$} & \multirow[t]{2}{*}{$\begin{array}{l}\text { Depth } \\
\text { of } \\
\text { well } 1 \\
\text { (ft) }\end{array}$} & \multirow[t]{2}{*}{$\begin{array}{l}\text { Depth } \\
\text { to } \\
\text { refusa } 1 \\
\text { (ft) }\end{array}$} & \multirow[t]{2}{*}{$\begin{array}{l}\text { Depth } \\
\text { to } \\
\text { top } \\
\text { (ft) }\end{array}$} & \multirow[t]{2}{*}{$r$} & \multirow[t]{2}{*}{ L1thology } \\
\hline & & & & & & & \\
\hline $\mathrm{NXW}$ & 17 & 122 & 122 & -- & -- & BEDROCK & \\
\hline NXW & 18 & 610 & 610 & -- & $\begin{array}{l}0 \\
4\end{array}$ & $\begin{array}{l}\text { 110SDMN } \\
\text { BEDROCK }\end{array}$ & SDGL \\
\hline NXW & 19 & 439 & 439 & -- & $\begin{array}{l}0 \\
5\end{array}$ & $\begin{array}{l}\text { 112TILL } \\
\text { BEDROCK }\end{array}$ & TIIL \\
\hline NXW & 20 & 235 & 235 & -- & $\begin{array}{r}0 \\
40\end{array}$ & $\begin{array}{l}\text { 112T ILI } \\
\text { BEDROCK }\end{array}$ & TILL \\
\hline NXW & 21 & 173 & 173 & -- & $\begin{array}{l}0 \\
3\end{array}$ & $\begin{array}{l}112 \text { TILL } \\
\text { BEDROCK }\end{array}$ & TILL \\
\hline NXW & 22 & 319 & 319 & -- & $\begin{array}{r}0 \\
16\end{array}$ & $\begin{array}{l}\text { 112TILL } \\
\text { BEDROCK }\end{array}$ & TILL \\
\hline NXW & 23 & 204 & 204 & -- & $\begin{array}{l}0 \\
5\end{array}$ & $\begin{array}{l}\text { 112TILL } \\
\text { BEDROCK }\end{array}$ & TILL \\
\hline NXW & 24 & 205 & 205 & -- & $\begin{array}{l}0 \\
8\end{array}$ & $\begin{array}{l}110 \text { SDMN } \\
\text { BEDROCK }\end{array}$ & SAND \\
\hline NXW & 25 & 322 & 322 & -- & $\begin{array}{c}0 \\
-- \\
25\end{array}$ & $\begin{array}{l}110 S D M N \\
112 T \text { ILL } \\
\text { BEDROCK }\end{array}$ & $\begin{array}{l}\text { SAND } \\
\text { TILL }\end{array}$ \\
\hline NXW & 26 & 182 & 182 & -- & $\begin{array}{r}0 \\
10\end{array}$ & $\begin{array}{l}\text { 112TILL } \\
\text { BEDROCK }\end{array}$ & TILL \\
\hline $\mathbf{N X W}$ & 27 & 300 & 300 & -- & $\begin{array}{r}0 \\
11\end{array}$ & $\begin{array}{l}110 \text { SDMN } \\
\text { BEDROCK }\end{array}$ & SDGL \\
\hline NXW & 28 & 144 & 144 & -- & $\begin{array}{r}0 \\
18\end{array}$ & $\begin{array}{l}\text { 112TILL } \\
\text { BEDROCK }\end{array}$ & TILL \\
\hline NXW & 29 & 310 & 310 & -- & 7 & BEDROCK & \\
\hline NXW & 30 & 280 & 280 & -- & $\begin{array}{l}0 \\
6\end{array}$ & $\begin{array}{l}\text { 110SDMN } \\
\text { BEDROCK }\end{array}$ & SAND \\
\hline $\mathbf{N X N}$ & 31 & 145 & 145 & -- & $\begin{array}{l}0 \\
8\end{array}$ & $\begin{array}{l}110 \text { SDMN } \\
\text { BEDROCK }\end{array}$ & SDGL \\
\hline NXW & 32 & 164 & 164 & -- & $\begin{array}{r}0 \\
12\end{array}$ & $\begin{array}{l}110 \text { SDMN } \\
\text { BEDROCK }\end{array}$ & SDGL \\
\hline NXW & 33 & 186 & 186 & -- & $\begin{array}{l}0 \\
4\end{array}$ & $\begin{array}{l}\text { 112TILL } \\
\text { BEDROCK }\end{array}$ & TILL \\
\hline NXH & 34 & 382 & 382 & -- & $\begin{array}{r}0 \\
10\end{array}$ & $\begin{array}{l}\text { 112TILL } \\
\text { BEDROCK }\end{array}$ & TILL \\
\hline NXN & 35 & 280 & 280 & -- & $\begin{array}{l}0 \\
9\end{array}$ & $\begin{array}{l}\text { 112T ILL } \\
\text { BEDROCK }\end{array}$ & TILL \\
\hline NXW & 36 & 223 & 223 & -- & $\begin{array}{r}0 \\
25\end{array}$ & $\begin{array}{l}\text { 110SDMN } \\
\text { BEDROCK }\end{array}$ & SDGL \\
\hline NXW & 37 & 202 & 202 & -- & $\begin{array}{r}0 \\
10\end{array}$ & $\begin{array}{l}\text { 112T ILL } \\
\text { BEDROCK }\end{array}$ & TILL \\
\hline $\mathrm{NXW}$ & 38 & 184 & 184 & -- & $\begin{array}{r}0 \\
25\end{array}$ & $\begin{array}{l}\text { 112TILL } \\
\text { BEDROCK }\end{array}$ & TILL \\
\hline
\end{tabular}


Table 3.--Lithologic logs of wells and borings--Continued

\begin{tabular}{|c|c|c|c|c|c|c|c|}
\hline \multicolumn{2}{|c|}{$\begin{array}{l}\text { Local } \\
\text { site } \\
\text { number }\end{array}$} & \multirow{3}{*}{$\begin{array}{l}\begin{array}{l}\text { Depth } \\
\text { driIIled } \\
(\mathrm{ft})\end{array} \\
\begin{array}{l}230\end{array}\end{array}$} & \multirow{3}{*}{$\begin{array}{c}\begin{array}{c}\text { Depth } \\
\text { of } \\
\text { well } \\
\text { (ft) }\end{array} \\
230\end{array}$} & \multirow{3}{*}{$\begin{array}{c}\begin{array}{c}\text { Depth } \\
\text { to } \\
\text { refusal } \\
\text { (ft) }\end{array} \\
\\
--\end{array}$} & \multirow[t]{2}{*}{$\begin{array}{l}\text { Depth } \\
\text { to } \\
\text { top } \\
(f t)\end{array}$} & \multirow{2}{*}{\multicolumn{2}{|c|}{$\begin{array}{c}\text { Lithology } \\
\text { am--Continued }\end{array}$}} \\
\hline & & & & & & & \\
\hline NXW & 40 & & & & $\begin{array}{r}0 \\
25\end{array}$ & $\begin{array}{l}110 \text { SDMN } \\
\text { BEDROCK }\end{array}$ & SDGL \\
\hline NXW & 42 & 300 & 300 & -- & $\begin{array}{r}0 \\
16\end{array}$ & $\begin{array}{l}110 \text { SDMN } \\
\text { BEDROCK }\end{array}$ & SDGL \\
\hline NXW & 43 & 180 & 180 & -- & $\begin{array}{r}0 \\
35\end{array}$ & $\begin{array}{l}110 \text { SDMN } \\
\text { BEDROCK }\end{array}$ & SAND \\
\hline NXW & 45 & 323 & 323 & -- & $\begin{array}{l}0 \\
7\end{array}$ & $\begin{array}{l}\text { 112TILL } \\
\text { BEDROCK }\end{array}$ & TILL \\
\hline NXW & 46 & 280 & 280 & -- & $\begin{array}{l}0 \\
9\end{array}$ & $\begin{array}{l}\text { 112TILL } \\
\text { BEDROCK }\end{array}$ & TILL \\
\hline NXW & 47 & 225 & 225 & -- & 20 & BEDROCK & \\
\hline NXW & 48 & 162 & 162 & -- & $\begin{array}{l}0 \\
2\end{array}$ & $\begin{array}{l}\text { 110SDMN } \\
\text { BEDROCK }\end{array}$ & SDGL \\
\hline NXW & 50 & 160 & 160 & -- & $\begin{array}{l}0 \\
7\end{array}$ & $\begin{array}{l}110 \text { SDMN } \\
\text { BEDROCK }\end{array}$ & SAND \\
\hline NXW & 55 & 190 & 190 & -- & $\frac{0}{10}$ & $\begin{array}{l}112 \text { TILL } \\
110 S D M N \\
\text { BEDROCK }\end{array}$ & $\begin{array}{l}\text { TILL } \\
\text { SDGL }\end{array}$ \\
\hline $\mathrm{NXW}$ & 56 & 225 & 225 & -- & $\begin{array}{l}0 \\
3\end{array}$ & $\begin{array}{l}110 \text { SDMN } \\
\text { BEDROCK }\end{array}$ & SAND \\
\hline NXW & 57 & 360 & 360 & -- & $\begin{array}{r}0 \\
20\end{array}$ & $\begin{array}{l}110 \text { SDMN } \\
\text { BEDROCK }\end{array}$ & SDGL \\
\hline NXW & 58 & 320 & 320 & -- & -- & BEDROCK & \\
\hline $\mathrm{NXW}$ & 59 & 275 & 275 & -- & $\begin{array}{r}0 \\
35\end{array}$ & $\begin{array}{l}112 \text { TILL } \\
\text { BEDROCK }\end{array}$ & TILL \\
\hline $\mathrm{NXW}$ & 60 & 195 & 195 & -- & $\begin{array}{r}0 \\
12\end{array}$ & $\begin{array}{l}\text { 110SDMN } \\
\text { BEDROCK }\end{array}$ & SDGL \\
\hline NXW & 61 & 365 & 365 & -- & $\begin{array}{r}0 \\
47\end{array}$ & $\begin{array}{l}112 \text { TILL } \\
\text { BEDROCK }\end{array}$ & TILL \\
\hline NXW & 62 & 200 & 200 & -- & $\begin{array}{l}0 \\
8\end{array}$ & $\begin{array}{l}110 S D M N \\
\text { BEDROCK }\end{array}$ & SDGL \\
\hline NXW & 63 & 142 & 142 & -- & $\begin{array}{r}0 \\
10\end{array}$ & $\begin{array}{l}110 \text { SDMN } \\
\text { BEDROCK }\end{array}$ & SDGL \\
\hline
\end{tabular}


Table 3.--Lithologic logs of wells and borings--Continued

\begin{tabular}{lllllll} 
& \multicolumn{1}{c}{ Depth } & Depth & Depth & & \\
Local & Depth & of & to & to & & \\
site & drillied & well & refusal & top & Aquifer & \\
number & $(f t)$ & $(f t)$ & $(f t)$ & (ft) & code & Lithology \\
\hline
\end{tabular}

Nottingham--Continued

\begin{tabular}{|c|c|c|c|c|c|c|c|}
\hline $\mathrm{NXW}$ & 64 & 240 & 240 & -- & $\begin{array}{r}0 \\
44\end{array}$ & $\begin{array}{l}112 \text { TILL } \\
\text { BEDROCK }\end{array}$ & TILL \\
\hline NXW & 65 & 365 & 365 & -- & $\begin{array}{l}0 \\
3\end{array}$ & $\begin{array}{l}110 \text { SDMN } \\
\text { BEDROCK }\end{array}$ & SAND \\
\hline NXW & 66 & 300 & 300 & -- & $\begin{array}{r}0 \\
15\end{array}$ & $\begin{array}{l}110 S D M N \\
\text { BEDROCK }\end{array}$ & SDGL \\
\hline NXW & 67 & 300 & 300 & -- & $\begin{array}{r}0 \\
25\end{array}$ & $\begin{array}{l}110 \text { SDMN } \\
\text { BEDROCK }\end{array}$ & SAND \\
\hline
\end{tabular}

Raymond

\begin{tabular}{|c|c|c|c|c|c|c|c|c|c|}
\hline $\mathrm{RBA}$ & 1 & 42 & -- & 42 & $\begin{array}{r}0 \\
9 \\
15 \\
16.5\end{array}$ & $\begin{array}{l}111 \text { FILL } \\
112 \text { SRFD } \\
112 \text { SRFD } \\
112 \text { TILL }\end{array}$ & $\begin{array}{l}\text { SDGL } \\
\text { SAND } \\
\text { SILT } \\
\text { TILL }\end{array}$ & $\begin{array}{l}\text { C, PEBBLES } \\
\text { VF, LIGHT BROWN } \\
\text { SANDY WITH SAND }\end{array}$ & LENSES \\
\hline RBB & 1 & 48 & 48 & -- & $\begin{array}{r}0 \\
3 \\
15 \\
26 \\
42 \\
48\end{array}$ & $\begin{array}{l}111 \mathrm{~F} \text { ILL } \\
112 \text { SRFD } \\
112 \text { SRFD } \\
112 \text { SRFD } \\
112 \text { TILL } \\
\text { BEDROCK }\end{array}$ & $\begin{array}{l}\text { SAND } \\
\text { SDGL } \\
\text { SAND } \\
\text { GRVL }\end{array}$ & $\begin{array}{l}\mathrm{M}-\mathrm{C} \\
\text { SANDY }\end{array}$ & \\
\hline $\mathrm{RBB}$ & 2 & 31 & 31 & - & $\begin{array}{r}0 \\
1 \\
4 \\
21 \\
31\end{array}$ & $\begin{array}{l}\text { 111SOIL } \\
112 \text { SRFD } \\
112 \text { SRFD } \\
112 \text { TLL } \\
\text { BEDROCK }\end{array}$ & $\begin{array}{l}\text { GRVL } \\
\text { SAND }\end{array}$ & & \\
\hline RBB & 3 & 28 & 28 & -- & $\begin{array}{r}0 \\
4 \\
17 \\
28\end{array}$ & $\begin{array}{l}111 \text { SWMP } \\
112 \text { SRFD } \\
112 \text { TILL } \\
\text { BEDROCK }\end{array}$ & $\begin{array}{l}\text { MUCK } \\
\text { GRVL }\end{array}$ & SANDY & \\
\hline RBB & 4 & 10 & 10 & -- & $\begin{array}{l}0 \\
2 \\
4\end{array}$ & $\begin{array}{l}111 \text { FILL } \\
112 \text { SRFD } \\
112 \text { SRFD }\end{array}$ & $\begin{array}{l}\text { OTHR } \\
\text { SAND } \\
\text { GRVL }\end{array}$ & $\begin{array}{l}\text { RIP-RAP } \\
\mathrm{C} \\
\mathrm{F}\end{array}$ & \\
\hline RBB & 5 & 13 & 13 & -- & $\begin{array}{r}0 \\
4 \\
13\end{array}$ & $\begin{array}{l}112 \text { SRFD } \\
112 \text { TILL } \\
\text { BEDROCK }\end{array}$ & GRVL & SANDY & \\
\hline RBW & 1 & 30 & 30 & 48 & 0 & 112SRED & SDGL & & \\
\hline RBW & 2 & -- & 59 & 36 & 0 & $112 \mathrm{OTSH}$ & SDGL & & \\
\hline RBW & 3 & 7.7 & 7.7 & 28 & 0 & $112 \mathrm{ICCC}$ & SDGL & & \\
\hline RBW & 4 & 8.1 & 8.1 & -- & 0 & $112 \mathrm{ICCC}$ & & & \\
\hline RBW & 5 & 8.2 & 8.2 & 13 & 0 & $112 \mathrm{ICCC}$ & & & \\
\hline RBW & 6 & 15 & 15 & -- & 0 & 112 ICCC & & & \\
\hline $\mathrm{RBW}$ & 7 & 11.9 & 11.9 & -- & 0 & $112 \mathrm{ICCC}$ & GRVL & & \\
\hline RBW & 8 & 38 & 37.2 & 38 & $\begin{array}{r}0 \\
22 \\
32\end{array}$ & $\begin{array}{l}112 \text { SRFD } \\
112 \text { SRFD } \\
112 \text { SRFD }\end{array}$ & $\begin{array}{l}\text { SAND } \\
\text { SDST } \\
\text { SDGL }\end{array}$ & $\begin{array}{l}\text { M } \\
\text { SILT AND F SAND } \\
\text { C SAND, E GRAVEL }\end{array}$ & \\
\hline
\end{tabular}


Table 3.--Lithologic logs of wells and borings--Continued

\begin{tabular}{|c|c|c|c|c|c|c|c|}
\hline \multicolumn{2}{|c|}{$\begin{array}{l}\text { Local } \\
\text { site } \\
\text { number }\end{array}$} & $\begin{array}{l}\text { Depth } \\
\text { drililed } \\
(\mathrm{ft})\end{array}$ & $\begin{array}{l}\text { Depth } \\
\text { of } \\
\text { well } \\
\text { (ft) }\end{array}$ & $\begin{array}{l}\text { Depth } \\
\text { to } \\
\text { refusal } \\
\text { (ft) }\end{array}$ & $\begin{array}{l}\text { Depth } \\
\text { to } \\
\text { top } \\
(\mathrm{ft})\end{array}$ & $\begin{array}{l}\text { Aquifer } \\
\text { code }\end{array}$ & Lithology \\
\hline & & & \multicolumn{5}{|c|}{ Raymond--Cont inued } \\
\hline RBW & 9 & 31.5 & 11 & -- & $\begin{array}{r}0 \\
24\end{array}$ & $\begin{array}{l}\text { 112SRFD } \\
\text { 112TILL }\end{array}$ & $\begin{array}{l}\text { SDGL C-F SAND, SOME PEBBLES } \\
\text { TILL }\end{array}$ \\
\hline RBW & 10 & 48 & 14 & -- & $\begin{array}{l}0 \\
14 \\
27 \\
38 \\
46\end{array}$ & $\begin{array}{l}\text { 112SRFD } \\
112 \text { SRFD } \\
112 \text { SRFD } \\
112 T I L L \\
\text { BEDROCK }\end{array}$ & $\begin{array}{l}\text { SAND C } \\
\text { SAND F-VF } \\
\text { SILT WITH F SAND } \\
\text { TILL } \\
\text { BRCC }\end{array}$ \\
\hline RBW & 11 & 28.8 & 21 & -- & $\begin{array}{r}0 \\
5 \\
22\end{array}$ & $\begin{array}{l}\text { 112SRFD } \\
112 \text { SRFD } \\
112 \text { TILL }\end{array}$ & $\begin{array}{l}\text { SDGL C SAND WITH PEBBLES } \\
\text { SAND C, WITH F LAYER AT } 9 \text { FT } \\
\text { TILL }\end{array}$ \\
\hline RBW & 12 & -- & 8 & - & -- & -- & \\
\hline RBW & 13 & -- & 11.2 & -- & -- & -- & \\
\hline RBW & 14 & -- & 13.3 & -- & -- & -- & \\
\hline RBW & 15 & -- & 290 & -- & -- & -- & \\
\hline RBW & 16 & -- & 14.5 & $\cdots$ & -- & -- & \\
\hline RBW & 17 & -- & 100 & -- & -- & BEDROCK & \\
\hline RBW & 19 & -- & 16.3 & -- & -- & -- & \\
\hline RBW & 20 & -- & 20 & -- & -- & -- & \\
\hline RBW & 21 & -- & 33 & -- & -- & -- & \\
\hline RBW & 22 & -- & 23 & -- & -- & -- & \\
\hline RBW & 23 & -- & 120 & -- & -- & BEDROCK & \\
\hline RBW & 24 & -- & 125 & -- & -- & -- & \\
\hline RBW & 25 & -- & 21 & -- & -- & -- & \\
\hline RBW & 26 & -- & 173 & -- & -- & BEDROCK & \\
\hline RBW & 27 & -- & 135 & -- & -- & -- & \\
\hline RBW & 28 & -- & 175 & -- & -- & BEDROCK & \\
\hline RBW & 30 & -- & 200 & -- & -- & BEDROCK & \\
\hline RBW & 31 & -- & 12 & -- & -- & -- & \\
\hline RBW & 33 & -- & 12 & -- & -- & -- & \\
\hline RBW & 34 & -- & 80 & -- & -- & -- & \\
\hline RBW & 35 & -- & 18 & -- & -- & -- & \\
\hline RBW & 36 & -- & 210 & -- & -- & BEDROCK & \\
\hline RBW & 37 & -- & 20 & -- & -- & -- & \\
\hline RBW & 39 & -- & 12 & -- & -- & -- & \\
\hline RBW & 41 & -- & 12 & -- & -- & -- & \\
\hline
\end{tabular}


Táble 3.--Lithologic logs of wells and borings--Continued

\begin{tabular}{|c|c|c|c|c|c|c|c|}
\hline \multicolumn{2}{|c|}{$\begin{array}{l}\text { Local } \\
\text { site } \\
\text { number }\end{array}$} & $\begin{array}{l}\text { Depth } \\
\text { drillled } \\
\text { (ft) }\end{array}$ & $\begin{array}{l}\text { Depth } \\
\text { of } \\
\text { well } \\
\text { (ft) }\end{array}$ & $\begin{array}{l}\text { Depth } \\
\text { to } \\
\text { refusal } \\
\text { (ft) }\end{array}$ & $\begin{array}{l}\text { Depth } \\
\text { to } \\
\text { top } \\
\text { (ft) }\end{array}$ & $\begin{array}{l}\text { Aquifer } \\
\text { code }\end{array}$ & Lithology \\
\hline & & & \multicolumn{5}{|c|}{ Raymond--Cont inued } \\
\hline RBW & 42 & -- & 20 & - & -- & -- & \\
\hline RBW & 46 & -- & 110 & -- & $\cdots$ & -- & \\
\hline RBW & 47 & -- & 114 & -- & -- & BEDROCK & \\
\hline RBW & 48 & 42 & 31.9 & 42 & $\begin{array}{r}0 \\
29\end{array}$ & $\begin{array}{l}\text { 112SRFD } \\
112 \text { SRFD }\end{array}$ & $\begin{array}{l}\text { SAND F-C } \\
\text { SDGL }\end{array}$ \\
\hline RBW & 58 & 201 & 201 & -- & $\begin{array}{r}0 \\
35\end{array}$ & $\begin{array}{l}110 S D M N \\
\text { BEDROCK }\end{array}$ & SDGL \\
\hline RBW & 59 & 24 & 24 & -- & -- & -- & \\
\hline RBW & 60 & 500 & 500 & -- & $\begin{array}{r}0 \\
75\end{array}$ & $\begin{array}{l}110 \text { SDMN } \\
\text { BEDROCK }\end{array}$ & SDGL \\
\hline RBW & 61 & 100 & 100 & -- & $\begin{array}{r}0 \\
12\end{array}$ & $\begin{array}{l}\text { 110SDMN } \\
\text { BEDROCK }\end{array}$ & SDGL \\
\hline RBW & 49 & 27 & 16 & -- & $\begin{array}{r}0 \\
12 \\
17 \\
22\end{array}$ & $\begin{array}{l}112 \text { SRFD } \\
112 \text { SRFD } \\
112 \text { SRFD } \\
112 \text { TILL }\end{array}$ & $\begin{array}{l}\text { SDGL M. SAND WITH PEBBLES } \\
\text { SAND M } \\
\text { SILT CLAYEY } \\
\text { TILL }\end{array}$ \\
\hline RBW & 50 & 19 & 17.1 & -- & $\begin{array}{r}0 \\
12 \\
17\end{array}$ & $\begin{array}{l}112 \text { SRFD } \\
112 \text { SRFD } \\
112 \text { TILL }\end{array}$ & $\begin{array}{l}\text { SDGL M-C SAND, PEBBLES } \\
\text { SAND M-C } \\
\text { TILL }\end{array}$ \\
\hline RBW & 51 & 73 & 20.7 & -- & $\begin{array}{l}0 \\
17 \\
68.5\end{array}$ & $\begin{array}{l}112 \text { SRFD } \\
112 \text { SRFD } \\
112 \text { TILL }\end{array}$ & $\begin{array}{l}\text { SDGL M-VC SAND, PEBBLES } \\
\text { SAND F } \\
\text { TILL }\end{array}$ \\
\hline RBW & 52 & 96 & 31.6 & $-\infty$ & $\begin{array}{r}0 \\
37 \\
58 \\
92\end{array}$ & $\begin{array}{l}112 \text { SRFD } \\
112 \text { SRFD } \\
112 \text { SRFD } \\
112 \text { TILL }\end{array}$ & $\begin{array}{l}\text { SDGL C SAND, WITH PEBBLES } \\
\text { SAND C-F SAND, SOME PEBBLES } \\
\text { SAND VF-F } \\
\text { TILL }\end{array}$ \\
\hline RBW & 53 & 140 & 140 & -- & $\frac{0}{12}$ & $\begin{array}{l}112 \text { TILL } \\
110 \text { SDMN } \\
\text { BEDROCK }\end{array}$ & $\begin{array}{l}\text { TILL } \\
\text { SDGL }\end{array}$ \\
\hline RBW & 54 & 140 & 140 & -- & $\begin{array}{l}0 \\
--\end{array}$ & $\begin{array}{l}112 \text { TILL } \\
110 S D M N \\
\text { BEDROCK }\end{array}$ & $\begin{array}{l}\text { TILL } \\
\text { SDGL }\end{array}$ \\
\hline RBW & 55 & 140 & 140 & -- & $\begin{array}{l}0 \\
-- \\
4\end{array}$ & $\begin{array}{l}110 \text { SDMN } \\
110 S D M N \\
\text { BEDROCK }\end{array}$ & $\begin{array}{l}\text { SAND } \\
\text { SDGL }\end{array}$ \\
\hline RBW & 56 & 240 & 240 & -- & $\frac{0}{12}$ & $\begin{array}{l}110 \text { SDMN } \\
110 \text { SDMN } \\
\text { BEDROCK }\end{array}$ & $\begin{array}{l}\text { SAND } \\
\text { SDGL }\end{array}$ \\
\hline RBW & 57 & 140 & 140 & -- & $\begin{array}{l}0 \\
5\end{array}$ & $\begin{array}{l}110 S D M N \\
\text { BEDROCK }\end{array}$ & SDGL \\
\hline RBW & 62 & 140 & 140 & -- & $\begin{array}{r}0 \\
19\end{array}$ & $\begin{array}{l}110 S D M N \\
\text { BEDROCK }\end{array}$ & SAND \\
\hline RBW & 63 & 240 & 240 & -- & $\begin{array}{r}0 \\
40\end{array}$ & $\begin{array}{l}110 S D M N \\
\text { BEDROCK }\end{array}$ & SAND \\
\hline
\end{tabular}


Table 3.--Lithologic logs of wells and borings--Continued

\begin{tabular}{|c|c|c|c|c|c|c|c|}
\hline \multicolumn{2}{|c|}{$\begin{array}{l}\text { Local } \\
\text { site } \\
\text { number }\end{array}$} & $\begin{array}{l}\text { Depth } \\
\text { drillled } \\
\text { (ft) }\end{array}$ & $\begin{array}{l}\text { Depth } \\
\text { of } \\
\text { well } \\
\text { (ft) }\end{array}$ & $\begin{array}{l}\text { Depth } \\
\text { to } \\
\text { refusal } \\
\text { (ft) }\end{array}$ & $\begin{array}{l}\text { Depth } \\
\text { to } \\
\text { top } \\
(f t)\end{array}$ & $\begin{array}{l}\text { Aquifer } \\
\text { code }\end{array}$ & Lithol \\
\hline & & & & & \multicolumn{3}{|c|}{ Raymond--Continue } \\
\hline RBW & 64 & 260 & 260 & -- & $\begin{array}{l}0 \\
7\end{array}$ & $\begin{array}{l}\text { 110SDMN } \\
\text { BEDROCK }\end{array}$ & SAND \\
\hline RBW & 65 & 200 & 200 & -- & $\begin{array}{r}0 \\
20\end{array}$ & $\begin{array}{l}110 \text { SDMN } \\
\text { BEDROCK }\end{array}$ & SAND \\
\hline RBW & 66 & 200 & 200 & -- & $\begin{array}{l}0 \\
8\end{array}$ & $\begin{array}{l}\text { 11OSDMN } \\
\text { BEDROCK }\end{array}$ & SAND \\
\hline RBW & 67 & 260 & 260 & -- & $\begin{array}{r}0 \\
16\end{array}$ & $\begin{array}{l}110 \text { SDMN } \\
\text { BEDROCK }\end{array}$ & SAND \\
\hline RBW & 68 & 240 & 240 & -- & $\begin{array}{r}0 \\
55\end{array}$ & $\begin{array}{l}110 \text { SDMN } \\
\text { BEDROCK }\end{array}$ & SAND \\
\hline RBW & 69 & 240 & 240 & -- & $\begin{array}{r}0 \\
20\end{array}$ & $\begin{array}{l}110 \text { SDMN } \\
\text { BEDROCK }\end{array}$ & SAND \\
\hline RBW & 70 & 180 & 180 & -- & $\begin{array}{l}0 \\
7\end{array}$ & $\begin{array}{l}110 \text { SDMN } \\
\text { BEDROCK }\end{array}$ & SDGL \\
\hline RBW & 71 & 180 & 180 & -- & $\begin{array}{r}0 \\
10\end{array}$ & $\begin{array}{l}110 \text { SDMN } \\
\text { BEDROCK }\end{array}$ & SAND \\
\hline RBW & 72 & 220 & 220 & -- & $\begin{array}{l}0 \\
5\end{array}$ & $\begin{array}{l}110 \text { SDMN } \\
\text { BEDROCK }\end{array}$ & SAND \\
\hline RBW & 73 & 200 & 200 & -- & $\begin{array}{l}0 \\
6\end{array}$ & $\begin{array}{l}110 \mathrm{SDMN} \\
\text { BEDROCK }\end{array}$ & SDGL \\
\hline RBW & 74 & 120 & 120 & -- & $\begin{array}{l}0 \\
8\end{array}$ & $\begin{array}{l}\text { 110SDMN } \\
\text { BEDROCK }\end{array}$ & SDGL \\
\hline RBW & 75 & 220 & 220 & -- & $\begin{array}{r}0 \\
27\end{array}$ & $\begin{array}{l}110 \text { SDMN } \\
\text { BEDROCK }\end{array}$ & SAND \\
\hline RBW & 76 & 240 & 240 & -- & $\begin{array}{r}0 \\
45\end{array}$ & $\begin{array}{l}110 \text { SDMN } \\
\text { BEDROCK }\end{array}$ & SAND \\
\hline RBW & 77 & 300 & 300 & -- & $\begin{array}{l}0 \\
8\end{array}$ & $\begin{array}{l}110 \text { SDMN } \\
\text { BEDROCK }\end{array}$ & SAND \\
\hline RBW & 78 & 225 & 225 & -- & $\begin{array}{l}0 \\
8\end{array}$ & $\begin{array}{l}110 \text { SDMN } \\
\text { BEDROCK }\end{array}$ & SDGL \\
\hline RBW & 79 & 266 & 266 & -- & $\begin{array}{l}0 \\
4\end{array}$ & $\begin{array}{l}110 \text { SDMN } \\
\text { BEDROCK }\end{array}$ & SDGL \\
\hline RBW & 80 & 220 & 220 & -- & $\begin{array}{l}0 \\
5\end{array}$ & $\begin{array}{l}110 \text { SDMN } \\
\text { BEDROCK }\end{array}$ & SAND \\
\hline RBW & 81 & 305 & 305 & -- & $\begin{array}{r}0 \\
12\end{array}$ & $\begin{array}{l}110 \text { SDMN } \\
\text { BEDROCK }\end{array}$ & SDGL \\
\hline RBW & 82 & 145 & 145 & -- & $\begin{array}{l}0 \\
7\end{array}$ & $\begin{array}{l}110 \text { SDMN } \\
\text { BEDROCK }\end{array}$ & SAND \\
\hline RBW & 83 & 120 & 120 & -- & $\begin{array}{r}0 \\
53\end{array}$ & $\begin{array}{l}110 \text { SDMN } \\
\text { BEDROCK }\end{array}$ & SDGL \\
\hline RBW & 84 & 235 & 235 & -- & $\begin{array}{r}0 \\
52\end{array}$ & $\begin{array}{l}\text { 110SDMN } \\
\text { BEDROCK }\end{array}$ & SDGL \\
\hline
\end{tabular}


Table 3.--Lithologic logs of wells and borings--Continued

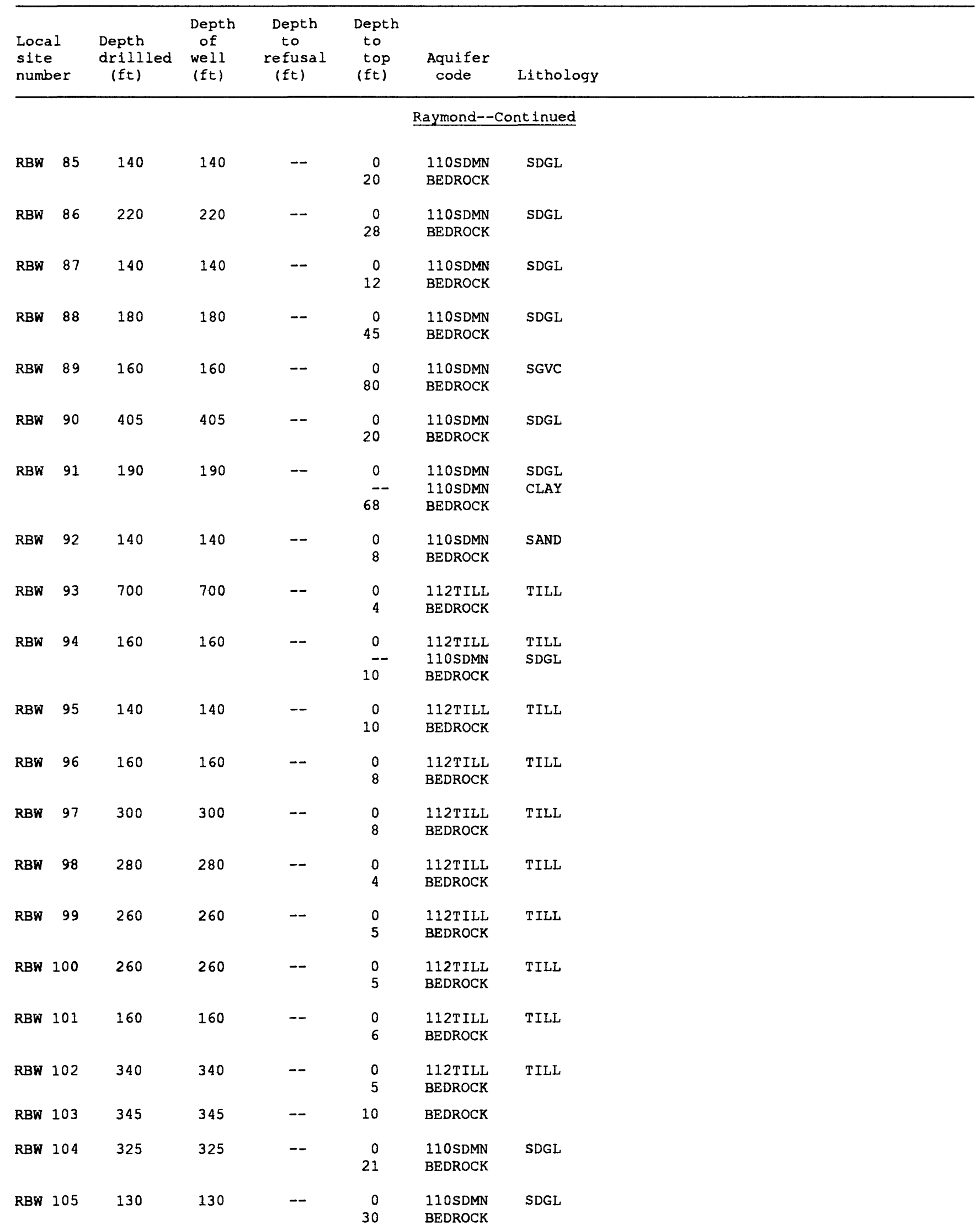


Table 3.--Lithologic logs of wells and borings--Continued

\begin{tabular}{|c|c|c|c|c|c|c|c|}
\hline \multicolumn{2}{|c|}{$\begin{array}{l}\text { Local } \\
\text { site } \\
\text { number }\end{array}$} & $\begin{array}{l}\text { Depth } \\
\text { drillled } \\
\quad(f t)\end{array}$ & $\begin{array}{l}\text { Depth } \\
\text { of } \\
\text { well } \\
\text { (ft) }\end{array}$ & $\begin{array}{l}\text { Depth } \\
\text { to } \\
\text { refusal } \\
\text { (ft) }\end{array}$ & $\begin{array}{l}\text { Depth } \\
\text { to } \\
\text { top } \\
(f t)\end{array}$ & $\begin{array}{l}\text { Aquifer } \\
\text { code }\end{array}$ & Iithology \\
\hline & & \multicolumn{6}{|c|}{ Sandown } \\
\hline SDA & 1 & 16 & -- & -- & $\begin{array}{r}0 \\
6 \\
16\end{array}$ & $\begin{array}{l}\text { 112SRFD } \\
112 \text { SRFD } \\
112 \text { TILL }\end{array}$ & $\begin{array}{l}\text { SDGL M-F SAND, GRANULES, COBBLES, PEBBLES } \\
\text { SAND F } \\
\text { TILL CLAY AND SILT MATRIX }\end{array}$ \\
\hline SDW & 3 & 83 & 42.2 & -- & $\begin{array}{r}0 \\
45 \\
72\end{array}$ & $\begin{array}{l}112 \text { SRFD } \\
112 \text { SRFD } \\
112 \text { SRFD }\end{array}$ & $\begin{array}{l}\text { SDGL MOSTLY M SAND } \\
\text { SAND VF WITH SILT } \\
\text { SAND M, SOME F }\end{array}$ \\
\hline SDW & 4 & -- & 300 & -- & -- & BEDROCK & \\
\hline SDW & 5 & -- & 12 & -- & -- & -- & \\
\hline SDW & 6 & -- & 870 & -- & -- & BEDROCK & \\
\hline SDW & 7 & -- & 380 & -- & -- & BEDROCK & \\
\hline SDW & 8 & -- & 210 & -- & -- & BEDROCK & \\
\hline SDW & 9 & -- & 18 & -- & -- & -- & \\
\hline SDW & 10 & -- & 20 & -- & -- & -- & \\
\hline SDW & 17 & -- & 150 & -- & -- & -- & \\
\hline SDW & 20 & -- & 11 & -- & -- & -- & \\
\hline SDW & 21 & -- & 150 & -- & -- & BEDROCK & \\
\hline SDW & 22 & -- & 12 & -- & -- & -- & \\
\hline SDW & 25 & -- & 500 & -- & -- & BEDROCK & \\
\hline SDW & 27 & -- & 120 & -- & -- & BEDROCK & \\
\hline SDW & 28 & -- & 80 & -- & -- & BEDROCK & \\
\hline SDW & 29 & -- & 30 & -- & -- & -- & \\
\hline SDW & 30 & -- & 250 & -- & -- & BEDROCK & \\
\hline SDW & 32 & -- & 75 & -- & - & -- & \\
\hline SDW & 34 & -- & 14 & -- & -- & -- & \\
\hline SDW & 37 & -- & 20 & -- & -- & -- & \\
\hline SDW & 38 & 114 & 103 & -- & $\begin{array}{r}0 \\
45 \\
72 \\
103\end{array}$ & $\begin{array}{l}112 \text { SRFD } \\
112 \text { SRFD } \\
112 \text { SRFD } \\
112 \text { SRFD }\end{array}$ & $\begin{array}{l}\text { SDGL M. SAND, WITH PEBBLES } \\
\text { SAND VF WITH SILT } \\
\text { SAND M, SOME F } \\
\text { SDGL F-C SAND, PEBBLES }\end{array}$ \\
\hline SDW & 39 & 220 & 220 & -- & $\begin{array}{r}0 \\
12\end{array}$ & $\begin{array}{l}112 \text { TILL } \\
\text { BEDROCK }\end{array}$ & TILL \\
\hline SDW & 40 & 150 & 150 & -- & $\begin{array}{r}0 \\
18\end{array}$ & $\begin{array}{l}\text { 112TILL } \\
\text { BEDROCK }\end{array}$ & TILL \\
\hline SDW & 41 & -- & 150 & -- & 26 & BEDROCK & ROCK -- \\
\hline SDW & 42 & $m$ & 112 & -- & 28 & BEDROCK & ROCK -- \\
\hline SDW & 43 & -- & 96 & -- & 12 & BEDROCK & ROCK -- \\
\hline SDW & 46 & 100 & 100 & -- & $\begin{array}{r}0 \\
12\end{array}$ & $\begin{array}{l}110 \text { SDMN } \\
\text { BEDROCK }\end{array}$ & SDGL \\
\hline
\end{tabular}


Table 3.--Lithologic logs of wells and borings--Continued

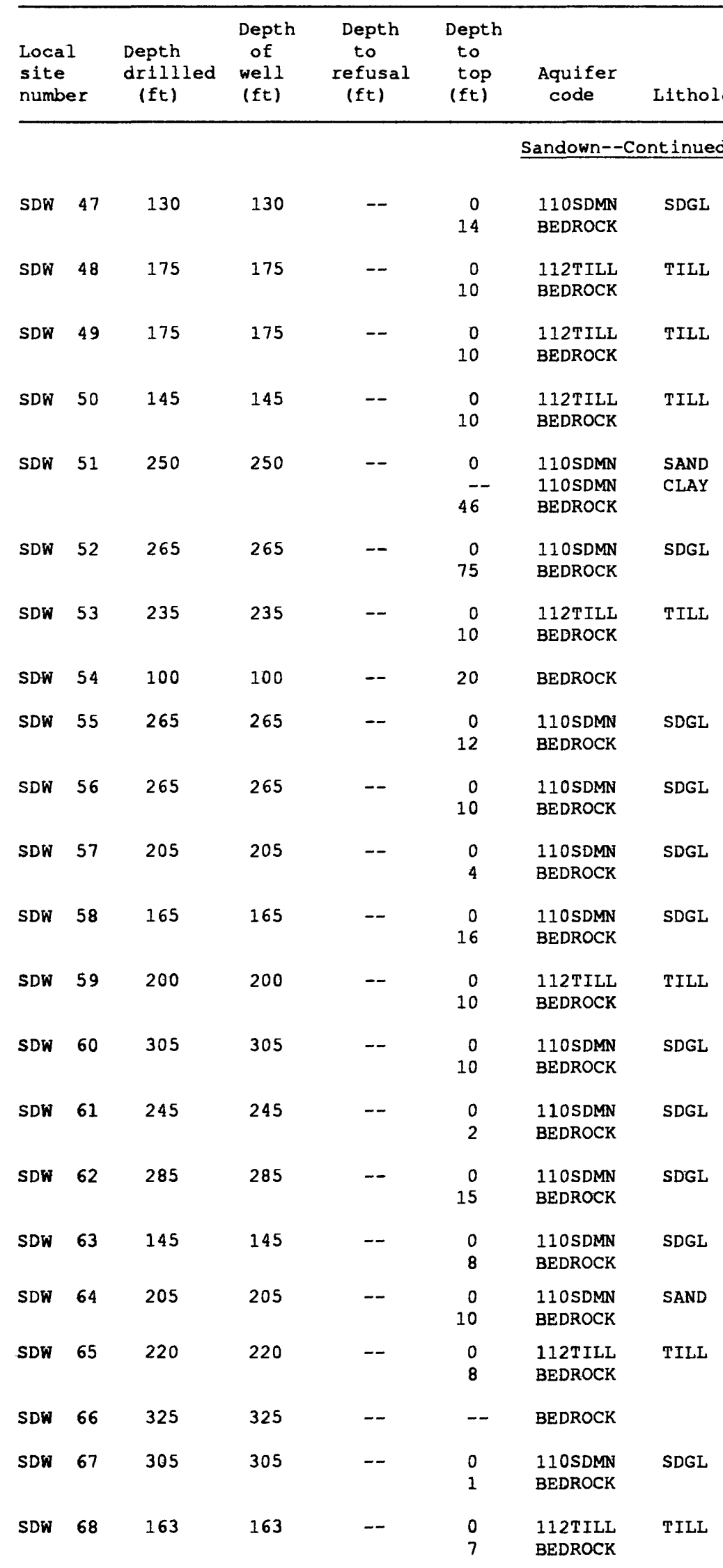


Table 3.--Lithologic logs of wells and borings--Continued

\begin{tabular}{|c|c|c|c|c|c|c|c|}
\hline \multicolumn{2}{|c|}{$\begin{array}{l}\text { Local } \\
\text { site } \\
\text { number }\end{array}$} & \multirow[t]{2}{*}{$\begin{array}{l}\text { Depth } \\
\text { drillled } \\
\text { (ft) }\end{array}$} & \multirow[t]{2}{*}{$\begin{array}{l}\text { Depth } \\
\text { of } \\
\text { well } \\
\text { (ft) }\end{array}$} & \multirow[t]{2}{*}{$\begin{array}{l}\text { Depth } \\
\text { to } \\
\text { refusal } \\
\text { (ft) }\end{array}$} & \multirow[t]{2}{*}{$\begin{array}{l}\text { Depth } \\
\text { to } \\
\text { top } \\
\text { (ft) }\end{array}$} & $\begin{array}{l}\text { Aquifer } \\
\text { code }\end{array}$ & \multirow[t]{2}{*}{ Lithology } \\
\hline \multicolumn{3}{|c|}{ Sandown--Cont inued } & & & & & \\
\hline SDW & 69 & 120 & 120 & -- & $\begin{array}{c}0 \\
--\end{array}$ & $\begin{array}{l}\text { 110SDMN } \\
\text { 112TILL } \\
\text { BEDROCK }\end{array}$ & $\begin{array}{l}\text { SDGL } \\
\text { TILL }\end{array}$ \\
\hline SDW & 70 & 160 & 160 & -- & $\begin{array}{r}0 \\
10\end{array}$ & $\begin{array}{l}112 \text { TILL } \\
\text { BEDROCK }\end{array}$ & TILL \\
\hline SDW & 71 & 225 & 225 & -- & $\begin{array}{r}0 \\
16\end{array}$ & $\begin{array}{l}\text { 11OSDMN } \\
\text { BEDROCK }\end{array}$ & SAND \\
\hline \multicolumn{8}{|c|}{ Stratham } \\
\hline SSB & 1 & 27 & 27 & -- & $\begin{array}{r}0 \\
1 \\
14 \\
27\end{array}$ & $\begin{array}{l}\text { 110SOIL } \\
112 \text { MRIN } \\
112 \text { SRFD } \\
\text { BEDROCK }\end{array}$ & $\begin{array}{l}\text { STCL -- } \\
\text { SAND GRAVELLY }\end{array}$ \\
\hline SSB & 2 & 69 & 69 & 69 & $\begin{array}{r}0 \\
22 \\
69\end{array}$ & $\begin{array}{l}\text { 110MRER } \\
112 \mathrm{MRIN} \\
112 \mathrm{TILL}\end{array}$ & $\begin{array}{l}\text { MUCK WITH CLAY, ORGANIC } \\
\text { SILT SOFT, WITH CLAY }\end{array}$ \\
\hline SSW & 1 & -- & 22 & -- & 0 & $112 \mathrm{SRFD}$ & SDGL \\
\hline ssw & 2 & -- & 100 & -- & -- & BEDROCK & \\
\hline SSW & 8 & -- & 25 & -- & 0 & $112 \mathrm{TILL}$ & TILL \\
\hline ssw & 9 & -- & 20.9 & -- & 0 & $112 \mathrm{SRFD}$ & SDGL \\
\hline SSW & 10 & -- & 21 & -- & 0 & $112 \mathrm{SRFD}$ & SDGL \\
\hline SSW & 12 & 310 & 310 & -- & $\begin{array}{r}0 \\
125\end{array}$ & $\begin{array}{l}\text { 112TILL } \\
\text { BEDROCK }\end{array}$ & TILL CLAY AND ROCKS \\
\hline SSW & 13 & 82 & 82 & -- & $\begin{array}{r}0 \\
62\end{array}$ & $\begin{array}{l}\text { 112SRFD } \\
\text { BEDROCK }\end{array}$ & SDGL "GRAVEL" \\
\hline ssw & 14 & 40 & 40 & -- & 26 & BEDROCK & \\
\hline sSW & 15 & 248 & 248 & -- & 100 & BEDROCK & \\
\hline ssw & 16 & 91 & 91 & -- & $\begin{array}{r}0 \\
28\end{array}$ & $\begin{array}{l}\text { 112SRFD } \\
\text { BEDROCK }\end{array}$ & SDGL SAND AND ROCKS \\
\hline ssw & 17 & -- & 57.5 & -- & -- & BEDROCK & \\
\hline SSW & 18 & 34 & 34 & -- & $\begin{array}{r}0 \\
10 \\
27 \\
34\end{array}$ & $\begin{array}{l}\text { 112SRFD } \\
112 \text { SRFD } \\
112 \text { TILL } \\
\text { BEDROCK }\end{array}$ & $\begin{array}{l}\text { SDGL } \\
\text { SAND F } \\
\text { TILL }\end{array}$ \\
\hline
\end{tabular}


Table 3.--Lithologic logs of wells and borings--Continued

\begin{tabular}{|c|c|c|c|c|c|c|c|}
\hline \multicolumn{2}{|c|}{$\begin{array}{l}\text { Local } \\
\text { site } \\
\text { number }\end{array}$} & $\begin{array}{l}\text { Depth } \\
\text { drillied } \\
(\mathrm{ft})\end{array}$ & $\begin{array}{l}\text { Depth } \\
\text { of } \\
\text { well } \\
(f t)\end{array}$ & $\begin{array}{l}\text { Depth } \\
\text { to } \\
\text { refusal } \\
\text { (ft) }\end{array}$ & $\begin{array}{l}\text { Depth } \\
\text { to } \\
\text { top } \\
(f t)\end{array}$ & $\begin{array}{l}\text { Aquifer } \\
\text { code }\end{array}$ & Lithology \\
\hline & & & \multicolumn{5}{|c|}{ Stratham--Continued } \\
\hline ssw & 19 & 34 & 34 & 34 & $\begin{array}{r}0 \\
30\end{array}$ & $\begin{array}{l}\text { 112SRED } \\
\text { 112TILL }\end{array}$ & $\begin{array}{l}\text { SDGL SILTY SAND SOME GRAVEL } \\
\text { TILL }\end{array}$ \\
\hline sSW & 22 & 29 & 29 & 29 & $\begin{array}{r}0 \\
21\end{array}$ & $\begin{array}{l}\text { 112SRFD } \\
\text { 112SRFD }\end{array}$ & $\begin{array}{l}\text { SDGL SILTY, BROWN } \\
\text { GRVL SILTY }\end{array}$ \\
\hline sSW & 23 & 30 & 30 & 30 & $\begin{array}{r}0 \\
21\end{array}$ & $\begin{array}{l}\text { 112SRFD } \\
\text { 112SRFD }\end{array}$ & $\begin{array}{l}\text { SDGL BROWN } \\
\text { SGVC BROWN }\end{array}$ \\
\hline SSW & 24 & 30 & 30 & 30 & 0 & 112SRFD & GRVL SILTY, SOME C SAND \\
\hline SSW & 25 & 4 & 4 & -- & -- & -- & \\
\hline SSW & 26 & 17.3 & 17.3 & 17.3 & 0 & 112SRFD & CLSD BROWN, F \\
\hline sSW & 27 & 24 & 24 & 24 & 0 & 112SRFD & SNCL GRAY-BROWN \\
\hline sSW & 28 & 18 & 18 & 18 & $\begin{array}{r}0 \\
14\end{array}$ & $\begin{array}{l}\text { 112SRFD } \\
112 \mathrm{TILL}\end{array}$ & $\begin{array}{l}\text { SNCL BROWN, } F \\
\text { TILL }\end{array}$ \\
\hline ssw & 29 & 23 & 23 & 23 & $\begin{array}{r}0 \\
21\end{array}$ & $\begin{array}{l}\text { 112SRFD } \\
112 \text { TILL }\end{array}$ & $\begin{array}{l}\text { SNCL BROWN, F } \\
\text { TILL }\end{array}$ \\
\hline sSw & 30 & 120 & 120 & -- & $\begin{array}{r}0 \\
30\end{array}$ & $\begin{array}{l}\text { 110SDMN } \\
\text { BEDROCK }\end{array}$ & SDGL \\
\hline SSW & 31 & 120 & 120 & - & $\begin{array}{r}0 \\
30\end{array}$ & $\begin{array}{l}\text { 110SDMN } \\
\text { BEDROCK }\end{array}$ & SDGL \\
\hline SSW & 32 & 1.70 & 170 & -- & -- & BEDROCK & \\
\hline ssw & 33 & 120 & 120 & -- & $\begin{array}{r}0 \\
20\end{array}$ & $\begin{array}{l}\text { 110SDMN } \\
\text { BEDROCK }\end{array}$ & SAND \\
\hline SSW & 34 & 240 & 240 & -- & $\begin{array}{r}0 \\
21\end{array}$ & $\begin{array}{l}\text { 110SDMN } \\
\text { BEDROCK }\end{array}$ & SAND \\
\hline SSW & 35 & 100 & 100 & -- & $\begin{array}{l}0 \\
6\end{array}$ & $\begin{array}{l}\text { 110SDMN } \\
\text { BEDROCK }\end{array}$ & SDGL \\
\hline SSW & 36 & 120 & 120 & -- & $\begin{array}{r}0 \\
18\end{array}$ & $\begin{array}{l}110 \text { SDMN } \\
\text { BEDROCK }\end{array}$ & SAND \\
\hline SSW & 38 & 120 & 120 & -- & $\begin{array}{r}0 \\
20\end{array}$ & $\begin{array}{l}\text { 110SDMN } \\
\text { BEDROCK }\end{array}$ & SDGL \\
\hline ssw & 39 & 202 & 202 & -- & $\begin{array}{r}0 \\
15\end{array}$ & $\begin{array}{l}110 S D M N \\
\text { BEDROCK }\end{array}$ & CLAY \\
\hline SSW & 40 & 200 & 200 & -- & $\begin{array}{l}0 \\
9\end{array}$ & $\begin{array}{l}\text { 110SDMN } \\
\text { BEDROCK }\end{array}$ & OTHR \\
\hline SSW & 41 & 100 & 100 & -- & $\begin{array}{l}0 \\
5\end{array}$ & $\begin{array}{l}110 \text { SDMN } \\
\text { BEDROCK }\end{array}$ & CLAY \\
\hline SSW & 42 & 300 & 300 & -- & $\begin{array}{r}0 \\
30\end{array}$ & $\begin{array}{l}110 \text { SDMN } \\
\text { BEDROCK }\end{array}$ & SAND \\
\hline SSW & 43 & 140 & 140 & -- & $\begin{array}{r}0 \\
15\end{array}$ & $\begin{array}{l}110 \text { SDMN } \\
\text { BEDROCK }\end{array}$ & SAND \\
\hline SSW & 44 & 320 & 320 & -- & $\begin{array}{r}0 \\
12\end{array}$ & $\begin{array}{l}110 S D M N \\
\text { BEDROCK }\end{array}$ & CLAY \\
\hline
\end{tabular}


Table 3.--Lithologic logs of wells and borings--Continued

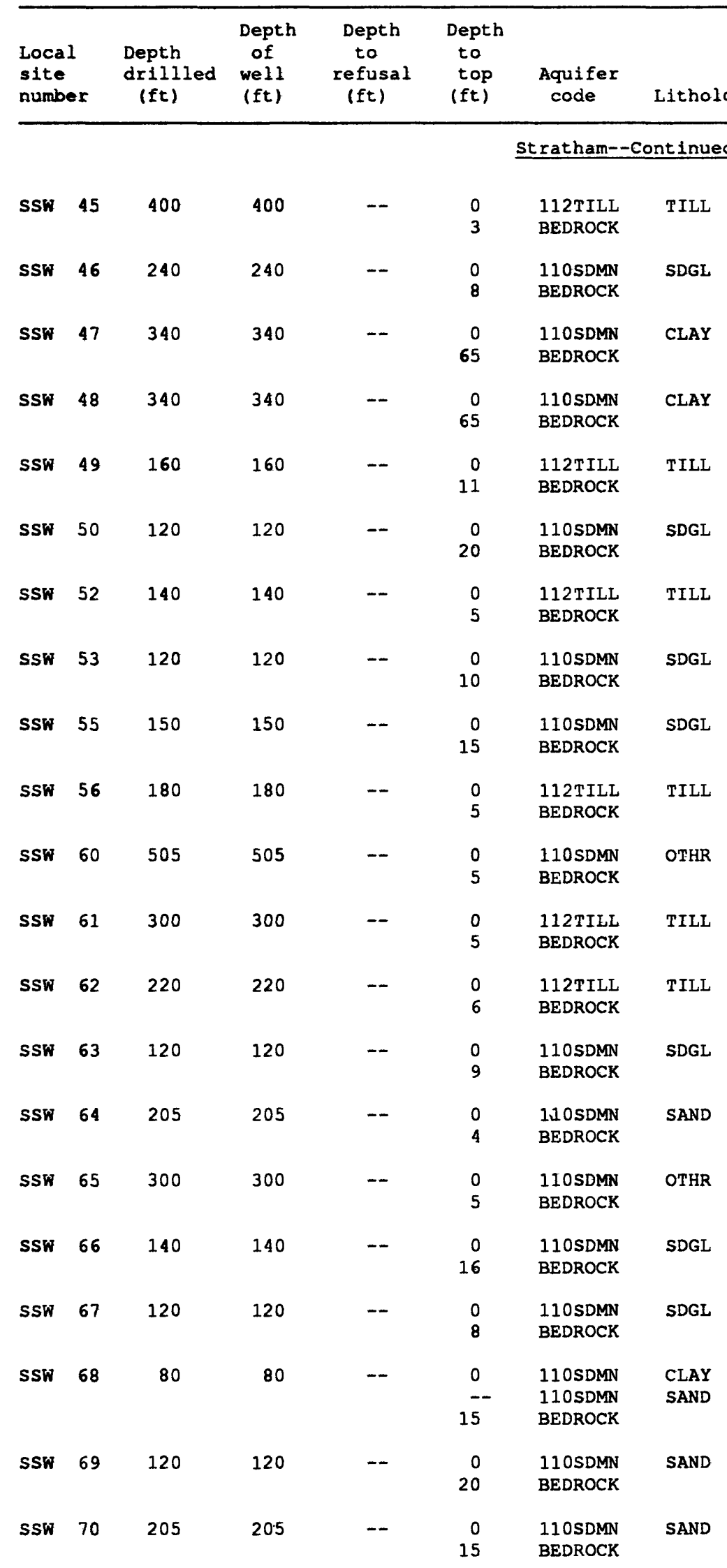


Table 3.--Lithologic logs of wells and borings--Continued

\begin{tabular}{|c|c|c|c|c|c|c|c|}
\hline \multicolumn{2}{|c|}{$\begin{array}{l}\text { Local } \\
\text { site } \\
\text { number }\end{array}$} & $\begin{array}{l}\text { Depth } \\
\text { drillled } \\
\text { (ft) }\end{array}$ & $\begin{array}{l}\text { Depth } \\
\text { of } \\
\text { well } \\
\text { (ft) }\end{array}$ & $\begin{array}{l}\text { Depth } \\
\text { to } \\
\text { refusal } \\
\text { (ft) }\end{array}$ & $\begin{array}{l}\text { Depth } \\
\text { to } \\
\text { top } \\
\text { (ft) }\end{array}$ & $\begin{array}{l}\text { Aquifer } \\
\text { code }\end{array}$ & Lithology \\
\hline & & & & & \multicolumn{3}{|c|}{ Stratham--Continued } \\
\hline SSW & 71 & 60 & 60 & -- & $\begin{array}{r}0 \\
15\end{array}$ & $\begin{array}{l}110 \text { SDMN } \\
\text { BEDROCK }\end{array}$ & SAND \\
\hline SSW & 72 & 120 & 120 & -- & $\frac{0}{15}$ & $\begin{array}{l}110 S D M N \\
110 S D M N \\
\text { BEDROCK }\end{array}$ & $\begin{array}{l}\text { SAND } \\
\text { CLAY }\end{array}$ \\
\hline SSW & 73 & 180 & 180 & -- & $\begin{array}{r}0 \\
15\end{array}$ & $\begin{array}{l}110 \text { SDMN } \\
\text { BEDROCK }\end{array}$ & SAND \\
\hline SSW & 74 & 100 & 100 & -- & $\begin{array}{r}0 \\
35\end{array}$ & $\begin{array}{l}110 \text { SDMN } \\
\text { BEDROCK }\end{array}$ & CLAY \\
\hline SSW & 75 & $\therefore$ & -- & -- & $\begin{array}{r}0 \\
15\end{array}$ & $\begin{array}{l}\text { 112TILL } \\
\text { BEDROCK }\end{array}$ & TILL \\
\hline SSW & 76 & 140 & 140 & -- & $\begin{array}{c}0 \\
30\end{array}$ & $\begin{array}{l}110 S D M N \\
110 S D M N \\
\text { BEDROCK }\end{array}$ & $\begin{array}{l}\text { SDGL } \\
\text { CLAY }\end{array}$ \\
\hline SSW & 78 & -- & 220 & -- & $\begin{array}{r}0 \\
20 \\
30\end{array}$ & $\begin{array}{l}110 \text { SDMN } \\
110 \text { SDMN } \\
\text { BEDROCK }\end{array}$ & $\begin{array}{l}\text { GRVL Gravel } \\
\text { CLAY } \\
\text { ROCK }\end{array}$ \\
\hline SSW & 79 & 100 & 100 & -- & $\begin{array}{l}0 \\
3\end{array}$ & $\begin{array}{l}110 S D M N \\
\text { BEDROCK }\end{array}$ & CLAY \\
\hline SSW & 80 & 160 & 160 & -- & $\begin{array}{r}0 \\
12\end{array}$ & $\begin{array}{l}112 \mathrm{TILL} \\
\text { BEDROCK }\end{array}$ & TILL \\
\hline SSW & 81 & -- & 201 & -- & $\begin{array}{r}0 \\
25 \\
50\end{array}$ & $\begin{array}{l}\text { 110SDMN } \\
112 \text { MRIN } \\
\text { BEDROCK }\end{array}$ & $\begin{array}{l}\text { GRVL Gravel } \\
\text { CLAY } \\
\text { ROCK }\end{array}$ \\
\hline SSW & 82 & 200 & 200 & -- & $\begin{array}{c}0 \\
--\end{array}$ & $\begin{array}{l}110 \text { SDMN } \\
110 \text { SDMN } \\
\text { BEDROCK }\end{array}$ & $\begin{array}{l}\text { SDGL } \\
\text { CLAY }\end{array}$ \\
\hline SSW & 83 & 240 & 240 & -- & $\begin{array}{c}0 \\
44\end{array}$ & $\begin{array}{l}110 \text { SDMN } \\
110 \text { SDMN } \\
\text { BEDROCK }\end{array}$ & $\begin{array}{l}\text { SAND } \\
\text { CLAY }\end{array}$ \\
\hline SSW & 84 & -- & 240 & -- & $\begin{array}{c}0 \\
--\end{array}$ & $\begin{array}{l}110 S D M N \\
110 S D M N \\
\text { BEDROCK }\end{array}$ & $\begin{array}{l}\text { SDGL } \\
\text { CLAY }\end{array}$ \\
\hline SSW & 85 & -- & 360 & -- & $\begin{array}{l}0 \\
-- \\
1\end{array}$ & $\begin{array}{l}110 \text { SDMN } \\
110 S D M N \\
\text { BEDROCK }\end{array}$ & $\begin{array}{l}\text { SDGL } \\
\text { CLAY }\end{array}$ \\
\hline SSW & 86 & -- & 80 & -- & $\begin{array}{r}0 \\
30\end{array}$ & $\begin{array}{l}110 \text { SDMN } \\
\text { BEDROCK }\end{array}$ & SDGL \\
\hline SSW & 87 & -- & 140 & -- & $\begin{array}{r}0 \\
15 \\
30\end{array}$ & $\begin{array}{l}110 S D M N \\
112 M R I N \\
\text { BEDROCK }\end{array}$ & $\begin{array}{l}\text { GRVL Gravel } \\
\text { CLAY } \\
\text { ROCK }\end{array}$ \\
\hline SSW & 88 & 302 & 302 & -- & $\begin{array}{c}0 \\
--\end{array}$ & $\begin{array}{l}110 S D M N \\
110 S D M N \\
\text { BEDROCK }\end{array}$ & $\begin{array}{l}\text { SDGL } \\
\text { CLAY }\end{array}$ \\
\hline SSW & 89 & 100 & 100 & -- & $\begin{array}{l}0 \\
3\end{array}$ & $\begin{array}{l}110 \text { SDMN } \\
\text { BEDROCK }\end{array}$ & SGVC \\
\hline
\end{tabular}


Table 3.--Lithologic logs of wells and borings--Continued

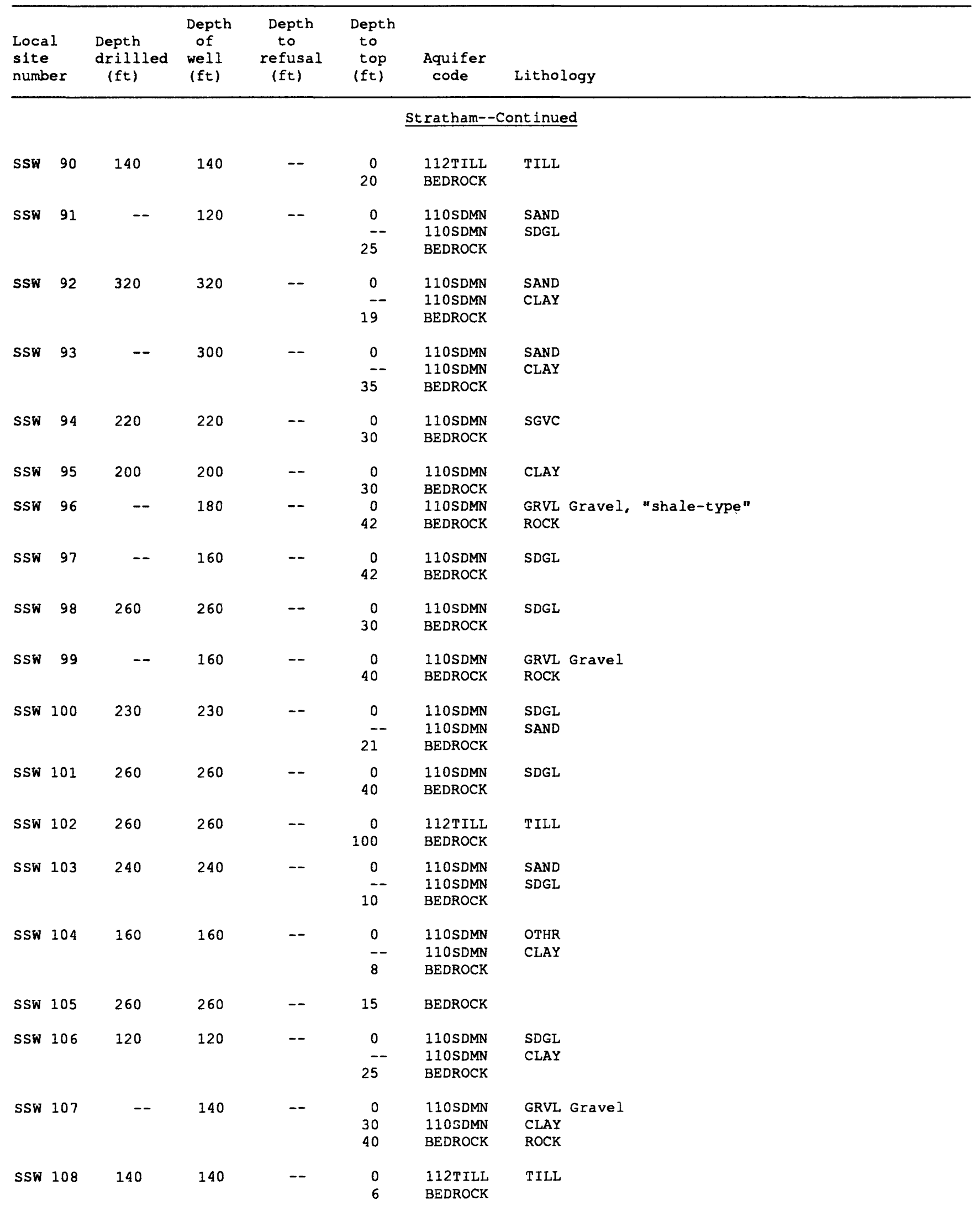


Table 3.--Lithologic logs of wells and borings--Continued

\begin{tabular}{|c|c|c|c|c|c|c|}
\hline $\begin{array}{l}\text { Local } \\
\text { site } \\
\text { number }\end{array}$ & $\begin{array}{l}\text { Depth } \\
\text { drillled } \\
(f t)\end{array}$ & $\begin{array}{l}\text { Depth } \\
\text { of } \\
\text { well } \\
(f t)\end{array}$ & $\begin{array}{l}\text { Depth } \\
\text { to } \\
\text { refusal } \\
\text { (ft) }\end{array}$ & $\begin{array}{l}\text { Depth } \\
\text { to } \\
\text { top } \\
\text { (ft) }\end{array}$ & $\begin{array}{l}\text { Aquifer } \\
\text { code }\end{array}$ & Lithology \\
\hline \multicolumn{7}{|c|}{ Stratham--Cont inued } \\
\hline SSW 109 & 160 & 160 & -- & $\frac{0}{30}$ & $\begin{array}{l}110 S D M N \\
110 S D M N \\
\text { BEDROCK }\end{array}$ & $\begin{array}{l}\text { SDGL } \\
\text { CLAY }\end{array}$ \\
\hline SSW 110 & -- & 140 & -- & $\begin{array}{r}0 \\
30\end{array}$ & $\begin{array}{l}\text { 11OSDMN } \\
\text { BEDROCK }\end{array}$ & $\begin{array}{l}\text { GRVL Gravel } \\
\text { ROCK }\end{array}$ \\
\hline SSW 112 & 320 & 320 & -- & $\begin{array}{l}0 \\
4\end{array}$ & $\begin{array}{l}\text { 110SDMN } \\
\text { BEDROCK }\end{array}$ & SAND \\
\hline SSW 113 & 160 & 160 & -- & $\begin{array}{r}0 \\
15\end{array}$ & $\begin{array}{l}\text { 110SDMN } \\
\text { BEDROCK }\end{array}$ & SDGL \\
\hline SSW 115 & 140 & 140 & -- & $\begin{array}{r}0 \\
13\end{array}$ & $\begin{array}{l}\text { 110SDMN } \\
\text { BEDROCK }\end{array}$ & SDGL \\
\hline SSW 116 & 400 & 400 & -- & $\begin{array}{l}0 \\
3\end{array}$ & $\begin{array}{l}\text { 112TILL } \\
\text { BEDROCK }\end{array}$ & TILL \\
\hline SSW 117 & 105 & 105 & -- & $\begin{array}{r}0 \\
25\end{array}$ & $\begin{array}{l}\text { 110SDMN } \\
\text { BEDROCK }\end{array}$ & SAND \\
\hline
\end{tabular}

STRAFFORD COUNTY

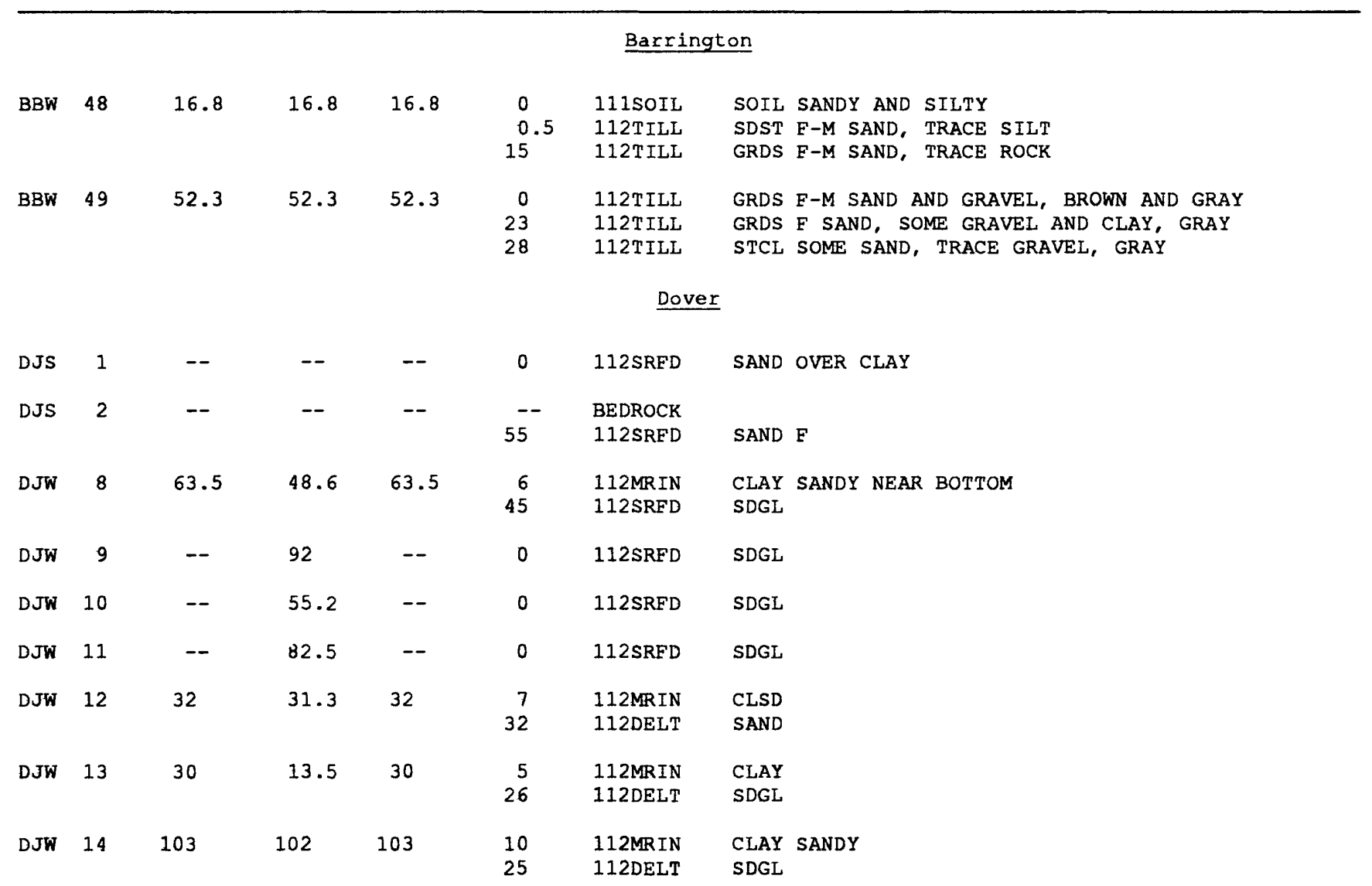


Table 3.--Lithologic logs of wells and borings--Continued

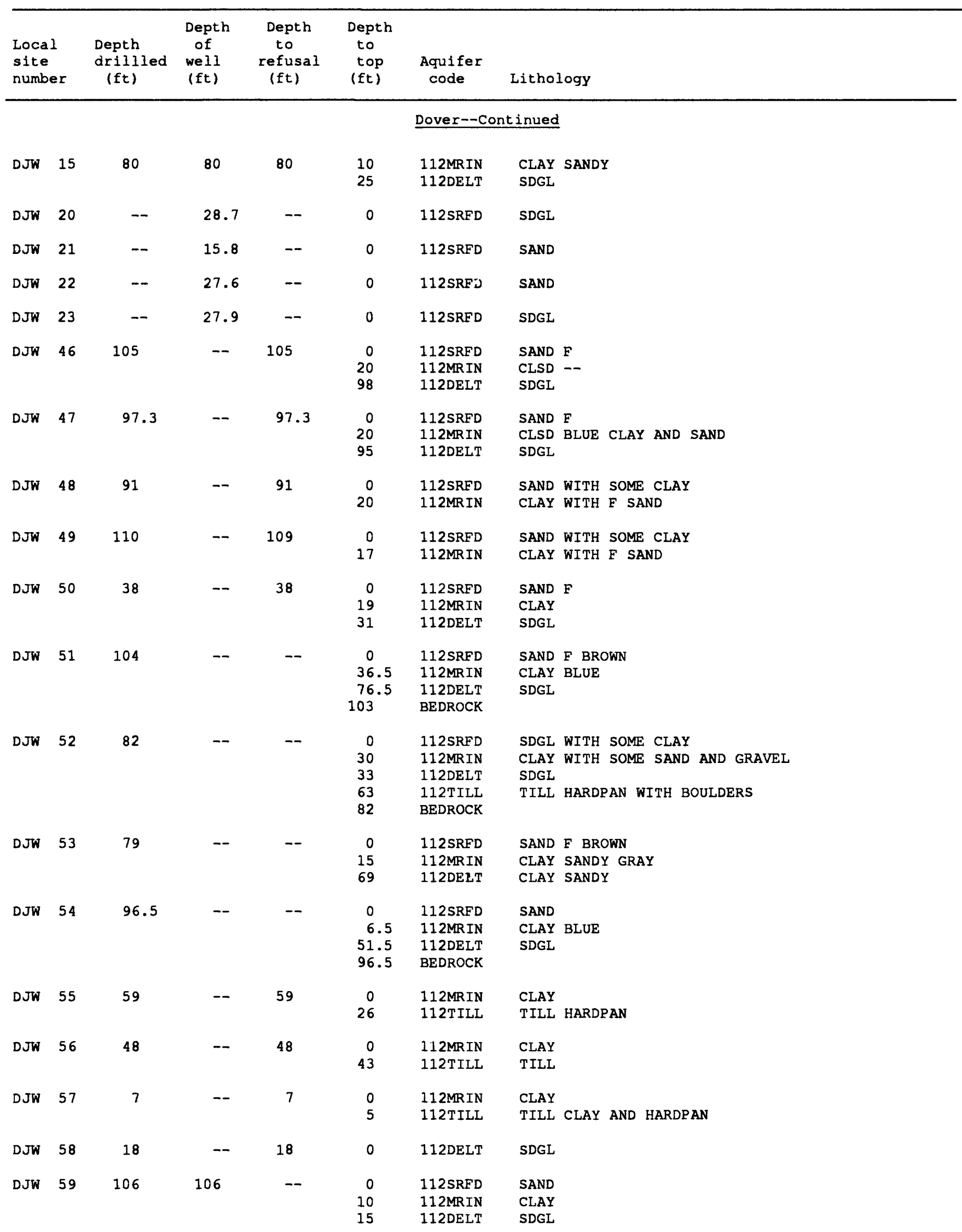


Table 3.--Lithologic logs of wells and borings--Continued

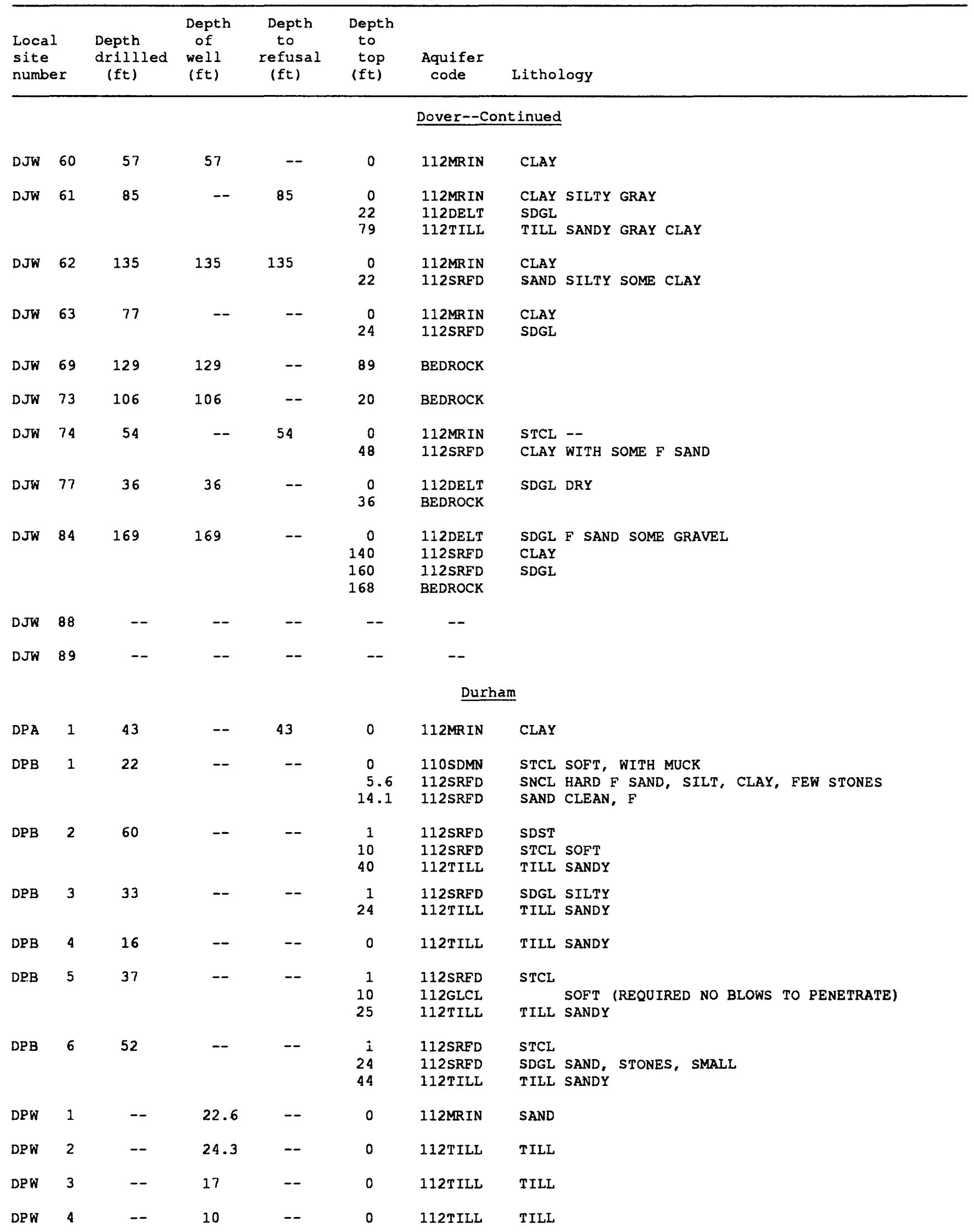


Table 3.--Lithologic logs of wells and borings--Continued

\begin{tabular}{|c|c|c|c|c|c|c|c|}
\hline \multicolumn{2}{|c|}{$\begin{array}{l}\text { Local } \\
\text { site } \\
\text { number }\end{array}$} & \multirow{3}{*}{$\begin{array}{l}\begin{array}{l}\text { Depth } \\
\text { drililed } \\
(f t)\end{array} \\
498\end{array}$} & \multirow{2}{*}{$\begin{array}{c}\text { Depth } \\
\text { of } \\
\text { well } \\
\text { (ft) }\end{array}$} & \multirow{3}{*}{$\begin{array}{c}\begin{array}{c}\text { Depth } \\
\text { to } \\
\text { refusal } \\
\text { (ft) }\end{array} \\
\\
--\end{array}$} & \multirow[t]{2}{*}{$\begin{array}{l}\text { Depth } \\
\text { to } \\
\text { top } \\
\text { (ft) }\end{array}$} & $\begin{array}{l}\text { Aquifer } \\
\text { code }\end{array}$ & \multirow[t]{2}{*}{ Lithology } \\
\hline & & & & & & Durham--Continued & \\
\hline DPW & 5 & & 498 & & $\begin{array}{r}0 \\
10\end{array}$ & $\begin{array}{l}\text { 112TILL } \\
\text { BEDROCK }\end{array}$ & TILL \\
\hline DPW & 6 & 550 & 550 & -- & 4 & BEDROCK & \\
\hline DPW & 7 & 195 & 195 & -- & $\begin{array}{r}0 \\
50 \\
55 \\
85\end{array}$ & $\begin{array}{l}\text { 112MRIN } \\
112 \text { SRFD } \\
\text { 112MRIN } \\
\text { BEDROCK }\end{array}$ & $\begin{array}{l}\text { CLAY } \\
\text { SAND F } \\
\text { CLAY }\end{array}$ \\
\hline DPW & 10 & 87.8 & 87.8 & -- & $\begin{array}{r}6 \\
51\end{array}$ & $\begin{array}{l}112 \text { SRFD } \\
112 \text { SRFD }\end{array}$ & $\begin{array}{l}\text { SDGL TRACE CLAY } \\
\text { SAND F WITH CLAY }\end{array}$ \\
\hline DPW & 11 & 47.5 & -- & 47.5 & $\begin{array}{r}4 \\
23 \\
27 \\
35 \\
40\end{array}$ & $\begin{array}{l}112 \mathrm{MRIN} \\
112 \mathrm{SRFD} \\
112 \mathrm{MRIN} \\
112 \text { SRFD } \\
112 \mathrm{TILL}\end{array}$ & $\begin{array}{l}\text { CLAY } \\
\text { SAND F BROWN } \\
\text { CLAY BLUE } \\
\text { SAND F BROWN } \\
\text { TILL }\end{array}$ \\
\hline DPW & 12 & 20.3 & -- & 20.3 & $\begin{array}{r}0 \\
10\end{array}$ & $\begin{array}{l}112 \mathrm{MRIN} \\
112 \mathrm{TILL}\end{array}$ & $\begin{array}{l}\text { CLAY FIRM } \\
\text { TILL CLAY AND BOULDERS }\end{array}$ \\
\hline DPW & 13 & 176 & 176 & -- & $\begin{array}{r}0 \\
50 \\
60\end{array}$ & $\begin{array}{l}\text { 112MRIN } \\
112 \text { SRFD } \\
\text { BEDROCK }\end{array}$ & $\begin{array}{l}\text { CLAY } \\
\text { SAND }\end{array}$ \\
\hline DPW & 17 & 445 & 445 & -- & $\begin{array}{r}0 \\
13\end{array}$ & $\begin{array}{l}\text { 11OSDMN } \\
\text { BEDROCK }\end{array}$ & SAND \\
\hline DPW & 18 & 180 & 180 & -- & 2 & BEDROCK & \\
\hline DPW & 19 & 170 & 170 & -- & $\begin{array}{r}0 \\
27\end{array}$ & $\begin{array}{l}\text { 110SDMN } \\
\text { BEDROCK }\end{array}$ & SGVC \\
\hline DPW & 20 & 240 & 240 & -- & $\begin{array}{r}0 \\
60\end{array}$ & $\begin{array}{l}\text { 110SDMN } \\
\text { BEDROCK }\end{array}$ & SAND \\
\hline DPW & 21 & 425 & 425 & -- & $\begin{array}{r}0 \\
98\end{array}$ & $\begin{array}{l}\text { 112TILL } \\
\text { BEDROCK }\end{array}$ & TILL \\
\hline DPW & 22 & 120 & 120 & -- & $\begin{array}{r}0 \\
30\end{array}$ & $\begin{array}{l}\text { 110SDMN } \\
\text { BEDROCK }\end{array}$ & SDGL \\
\hline DPW & 23 & 300 & 300 & -- & $\begin{array}{r}0 \\
15\end{array}$ & $\begin{array}{l}110 \text { SDMN } \\
\text { BEDROCK }\end{array}$ & SDGL \\
\hline DPW & 24 & 625 & 625 & -- & $\begin{array}{l}0 \\
8\end{array}$ & $\begin{array}{l}\text { 11OSDMN } \\
\text { BEDROCK }\end{array}$ & CLAY \\
\hline DPW & 25 & 124 & 124 & -- & $\begin{array}{r}0 \\
35\end{array}$ & $\begin{array}{l}\text { 11OSDMN } \\
\text { BEDROCK }\end{array}$ & CLAY \\
\hline
\end{tabular}


Table 3.--Lithologic logs of wells and borings--Continued

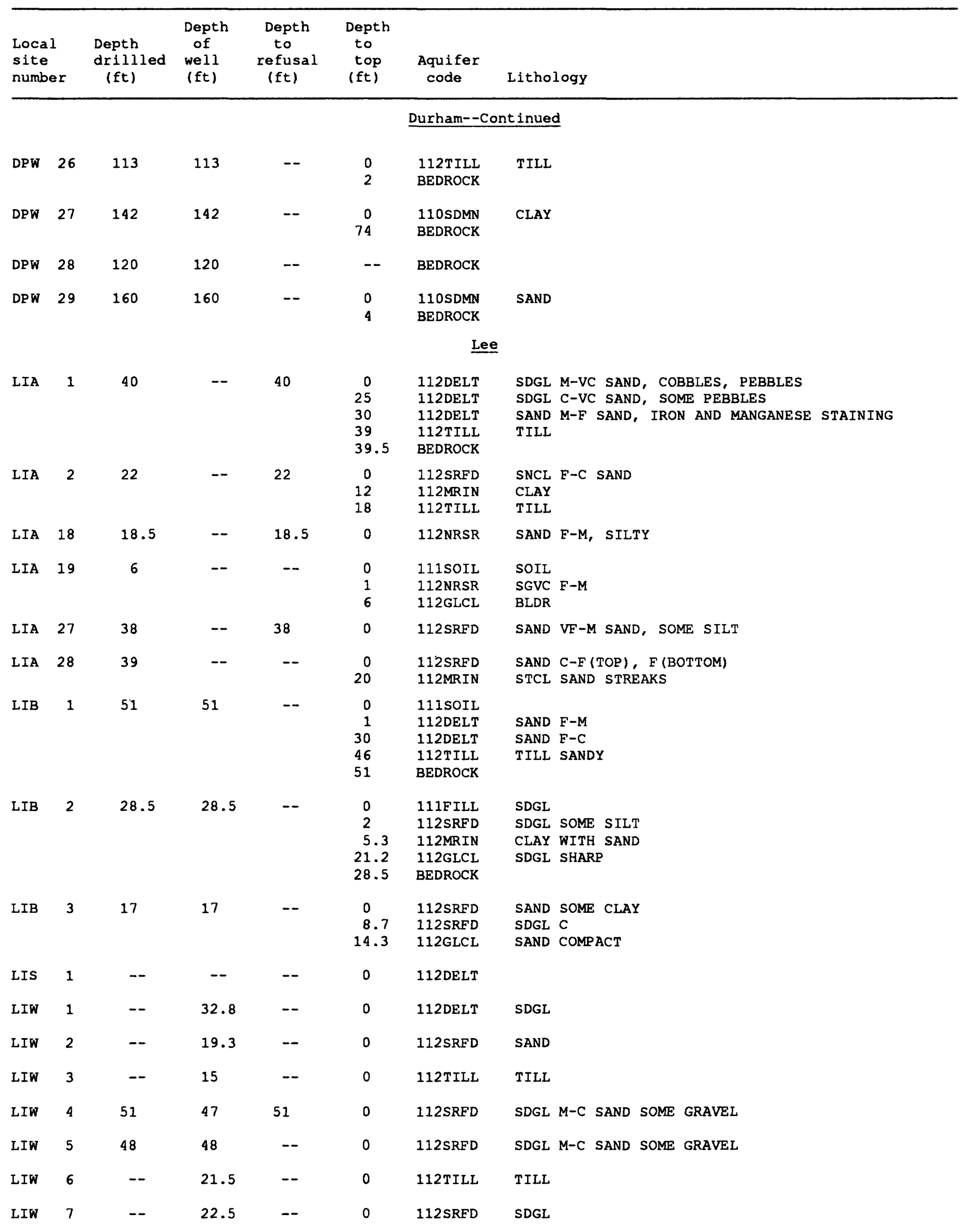


Table 3.--Lithologic logs of wells and borings--Continued

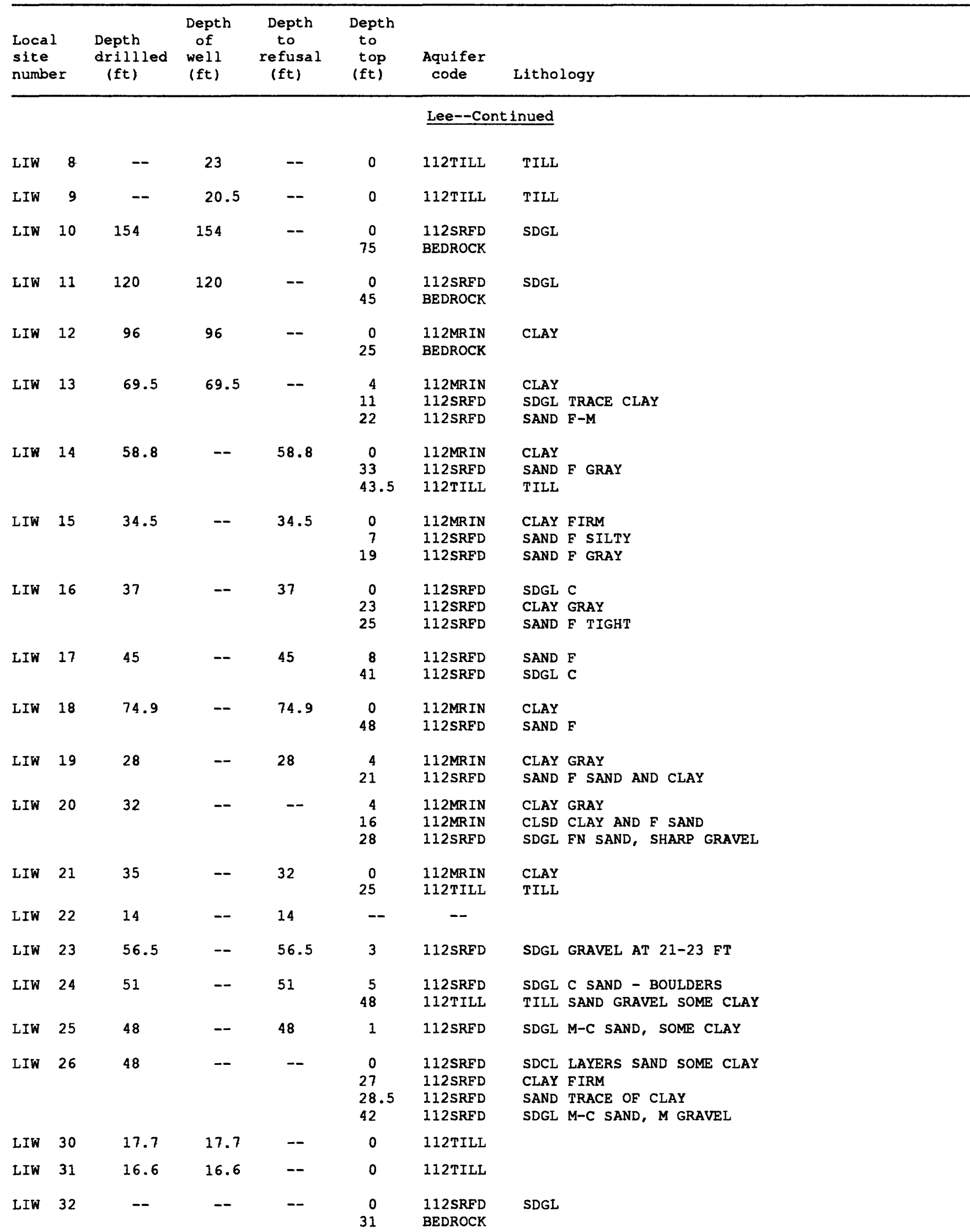


Table 3.--Lithologic logs of wells and borings--Continued

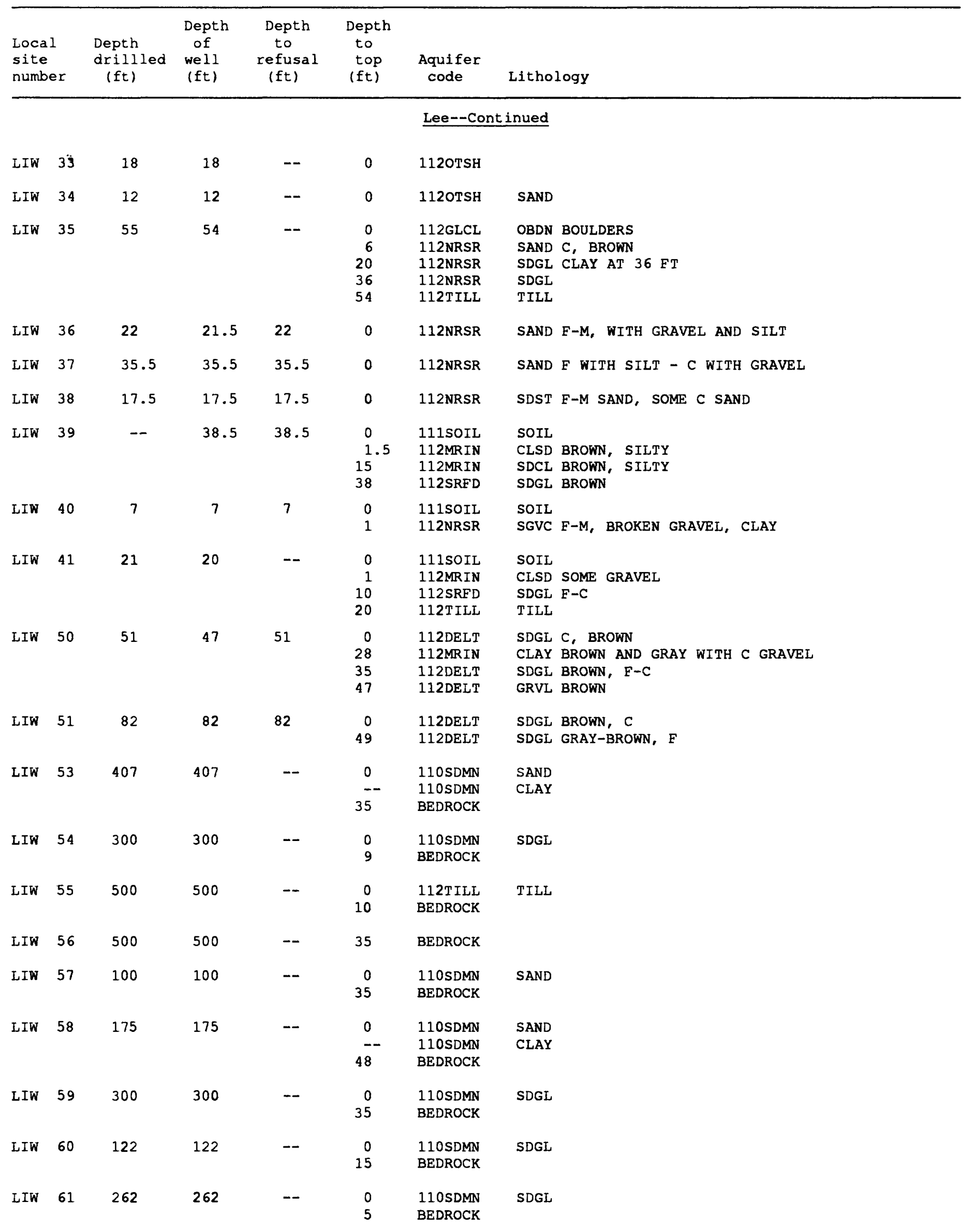


Table 3.--Lithologic logs of wells and borings--Continued

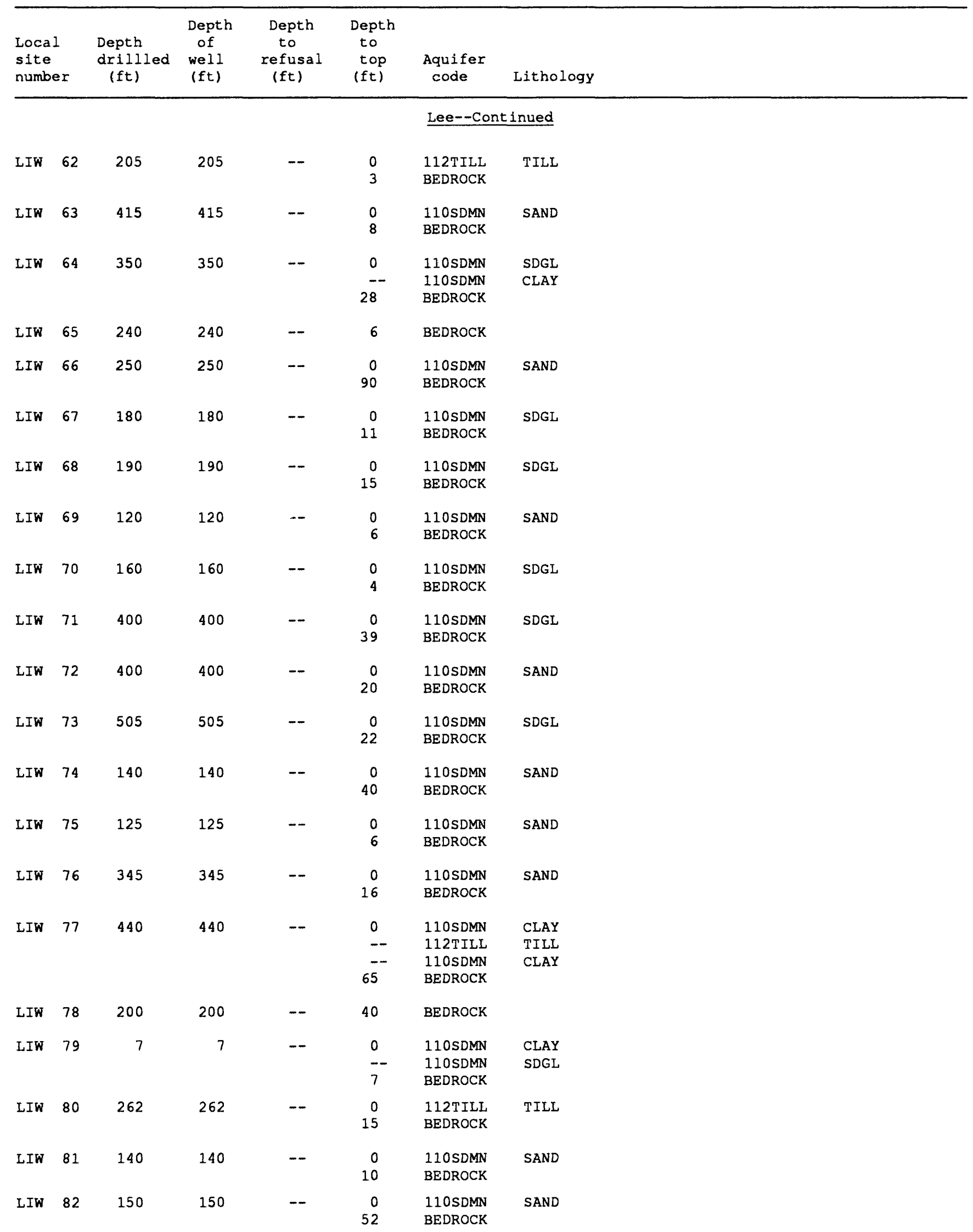


Table 3.--Lithologic logs of wells and borings--Continued

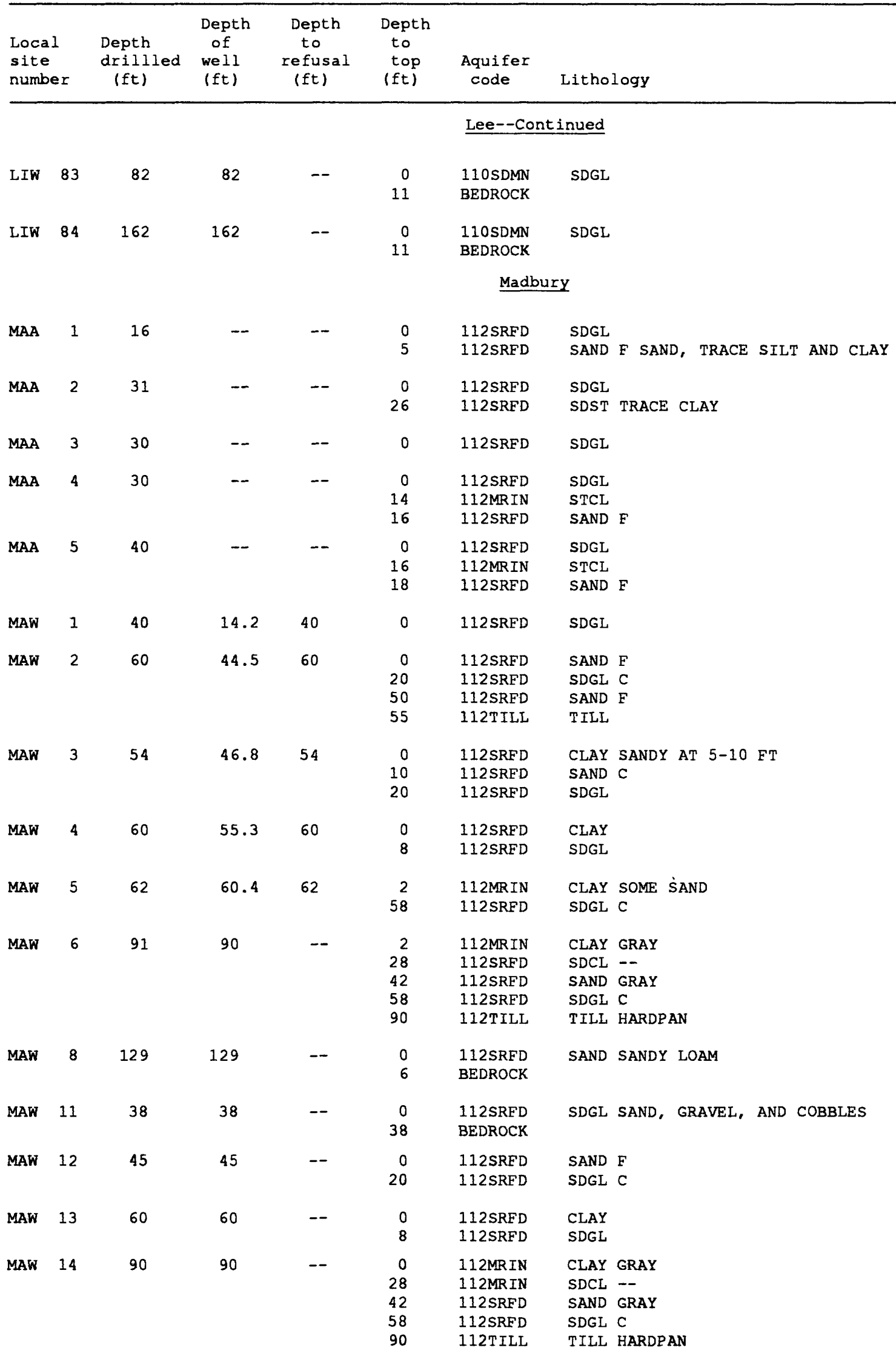


Table 3.--Lithologic logs of wells and borings--Continued

\begin{tabular}{|c|c|c|c|c|c|c|c|c|}
\hline \multicolumn{2}{|c|}{$\begin{array}{l}\text { Local } \\
\text { site } \\
\text { number }\end{array}$} & $\begin{array}{l}\text { Depth } \\
\text { drillled } \\
\text { (ft) }\end{array}$ & $\begin{array}{l}\text { Depth } \\
\text { of } \\
\text { well } \\
\text { (ft) }\end{array}$ & $\begin{array}{l}\text { Depth } \\
\text { to } \\
\text { refusal } \\
\text { (ft) }\end{array}$ & $\begin{array}{l}\text { Depth } \\
\text { to } \\
\text { top } \\
\text { (ft) }\end{array}$ & $\begin{array}{l}\text { Aquifer } \\
\text { code }\end{array}$ & Litho & $\log y$ \\
\hline & & & \multicolumn{6}{|c|}{ Madbury--Cont inued } \\
\hline MAW & 21 & 55 & -- & 55 & $\begin{array}{r}0 \\
43\end{array}$ & $\begin{array}{l}\text { 112MRIN } \\
\text { 112DELT }\end{array}$ & $\begin{array}{l}\text { CLAY } \\
\text { SAND }\end{array}$ & c \\
\hline MAW & 22 & 74 & -- & 74 & $\begin{array}{r}3 \\
55\end{array}$ & $\begin{array}{l}\text { 112MRIN } \\
\text { 112DELT }\end{array}$ & $\begin{array}{l}\text { CLAY } \\
\text { SAND }\end{array}$ & F \\
\hline MAW & 23 & 98 & -- & 55 & $\begin{array}{r}0 \\
34\end{array}$ & $\begin{array}{l}\text { 112MRIN } \\
\text { 112DELT }\end{array}$ & $\begin{array}{l}\text { CLAY } \\
\text { SAND }\end{array}$ & $\mathbf{F}$ \\
\hline MAW & 24 & 110 & -- & 45 & 0 & 112DELT & SDGL & \\
\hline MAW & 25 & 52 & -- & 52 & 0 & 112DELT & SAND & $\mathbf{F}$ \\
\hline MAN & 26 & 37.5 & 33.5 & 37.5 & $\begin{array}{r}4 \\
26\end{array}$ & $\begin{array}{l}\text { 112MRIN } \\
112 \text { DELT }\end{array}$ & $\begin{array}{l}\text { CLAY } \\
\text { SAND }\end{array}$ & C SAND, AND CLAY \\
\hline MAW & 27 & 45 & 45 & 45 & $\begin{array}{r}6 \\
23\end{array}$ & $\begin{array}{l}112 \mathrm{DELT} \\
112 \mathrm{DELT}\end{array}$ & $\begin{array}{l}\text { SDGL } \\
\text { GRVL }\end{array}$ & c \\
\hline MAW & 28 & 81 & 81 & 81 & $\begin{array}{r}6 \\
38 \\
58 \\
71\end{array}$ & $\begin{array}{l}112 \mathrm{DELT} \\
112 \mathrm{DELT} \\
112 \mathrm{DELT} \\
112 \mathrm{DELT}\end{array}$ & $\begin{array}{l}\text { SAND } \\
\text { SAND } \\
\text { SDGL } \\
\text { SAND }\end{array}$ & $\begin{array}{l}\text { F GRAY } \\
M-F, \text { BROWN } \\
\text { F GRAY }\end{array}$ \\
\hline MAN & 29 & 77.8 & -- & 77.8 & $\begin{array}{r}2 \\
24 \\
73\end{array}$ & $\begin{array}{l}112 \mathrm{MRIN} \\
112 \mathrm{SRFD} \\
112 \mathrm{DELT}\end{array}$ & $\begin{array}{l}\text { CLAY } \\
\text { CLAY } \\
\text { SAND }\end{array}$ & SANDY \\
\hline MAW & 30 & 98 & -- & 98 & $\begin{array}{r}0 \\
20 \\
70\end{array}$ & $\begin{array}{l}112 \mathrm{SRFD} \\
112 \mathrm{MRIN} \\
112 \mathrm{SRFD}\end{array}$ & $\begin{array}{l}\text { SAND } \\
\text { CLAY } \\
\text { SILT }\end{array}$ & SANDY \\
\hline MAN & 31 & 93 & -- & 93 & $\begin{array}{r}1 \\
32\end{array}$ & $\begin{array}{l}\text { 112SRFD } \\
112 \mathrm{SRFD}\end{array}$ & $\begin{array}{l}\text { CLSD } \\
\text { SAND }\end{array}$ & $\bar{F}$ \\
\hline MAW & 32 & 91 & -- & 91 & $\begin{array}{r}1 \\
24 \\
56\end{array}$ & $\begin{array}{l}\text { 112SRFD } \\
112 \mathrm{MRIN} \\
112 \text { SRFD }\end{array}$ & $\begin{array}{l}\text { SAND } \\
\text { CLAY } \\
\text { SAND }\end{array}$ & $F$ \\
\hline MAW & 33 & 70 & -- & 70 & $\begin{array}{r}1 \\
27 \\
44 \\
54\end{array}$ & $\begin{array}{l}112 \mathrm{MRIN} \\
112 \mathrm{SRFD} \\
112 \mathrm{SRFD} \\
112 \mathrm{SRFD}\end{array}$ & $\begin{array}{l}\text { CLSD } \\
\text { SDGL } \\
\text { GRVL } \\
\text { SDGL }\end{array}$ & $\begin{array}{l}\text { CLAY AND F SAND } \\
\text { GRAVEL AND C SAND }\end{array}$ \\
\hline MAW & 36 & 42 & - & 42 & $\begin{array}{r}0 \\
24\end{array}$ & $\begin{array}{l}112 \text { SRFD } \\
112 \text { MRIN }\end{array}$ & $\begin{array}{l}\text { SAND } \\
\text { CLAY }\end{array}$ & $F$ \\
\hline MAW & 37 & 37 & -- & 37 & $\begin{array}{r}2 \\
30\end{array}$ & $\begin{array}{l}\text { 112MRIN } \\
112 \text { TILL }\end{array}$ & $\begin{array}{l}\text { CLAY } \\
\text { TILL }\end{array}$ & $\begin{array}{l}\text { SANDY AT } 14-16 \mathrm{FT} \\
\text { HARDPAN }\end{array}$ \\
\hline MAW & 41 & 39 & -- & 39 & $\begin{array}{r}2 \\
25\end{array}$ & $\begin{array}{l}112 \mathrm{MRIN} \\
112 \mathrm{SRFD}\end{array}$ & $\begin{array}{l}\text { CLAY } \\
\text { SDGL }\end{array}$ & SAND, GRAVEL, AND CLAY \\
\hline MAW & 42 & 35 & -- & 35 & 1 & $112 \mathrm{MRIN}$ & CLAY & SANDY AT $12-35 \mathrm{FT}$ \\
\hline MAW & 43 & 82 & -- & 82 & $\begin{array}{r}2 \\
39 \\
76\end{array}$ & $\begin{array}{l}112 \mathrm{MRIN} \\
112 \mathrm{SRFD} \\
112 \mathrm{SRFD}\end{array}$ & $\begin{array}{l}\text { CLAY } \\
\text { SAND } \\
\text { SDGL }\end{array}$ & $\begin{array}{l}\text { F SAND AND CLAY } \\
\text { GRAVEL, SANDY }\end{array}$ \\
\hline MAW & 44 & 79 & -- & 79 & $\begin{array}{r}2 \\
62 \\
73\end{array}$ & $\begin{array}{l}\text { 112MRIN } \\
112 \text { SRFD } \\
112 \text { SRFD }\end{array}$ & $\begin{array}{l}\text { CLAY } \\
\text { SDGL } \\
\text { CLAY }\end{array}$ & $\begin{array}{l}\text { SANDY } \\
\text { C SAND, AND GRAVEL } \\
\text { CLAY AND F SAND }\end{array}$ \\
\hline MAW & 45 & 47 & -- & 47 & 0 & 112SRFD & SAND & $\mathbf{F}$ \\
\hline
\end{tabular}


Table 3.--Lithologic logs of wells and borings--Continued

\begin{tabular}{|c|c|c|c|c|c|c|c|}
\hline \multicolumn{2}{|c|}{$\begin{array}{l}\text { Local } \\
\text { site } \\
\text { number }\end{array}$} & \multirow[t]{2}{*}{$\begin{array}{l}\text { Depth } \\
\text { drililed } \\
\text { (ft) }\end{array}$} & \multirow[t]{2}{*}{$\begin{array}{l}\text { Depth } \\
\text { of } \\
\text { well } \\
(f t)\end{array}$} & \multirow[t]{2}{*}{$\begin{array}{l}\text { Depth } \\
\text { to } \\
\text { refusal } \\
\text { (ft) }\end{array}$} & \multirow[t]{2}{*}{$\begin{array}{l}\text { Depth } \\
\text { to } \\
\text { top } \\
\text { (ft) }\end{array}$} & $\begin{array}{l}\text { Aquifer } \\
\text { code }\end{array}$ & \multirow[t]{2}{*}{ Lithology } \\
\hline \multicolumn{3}{|c|}{ Madbury--Cont inued } & & & & & \\
\hline MAW : & 46 & 15 & -- & 15 & 1 & $112 \mathrm{TILL}$ & TILL SANDY CLAY, SOME GRAVEL \\
\hline MAW & 48 & 31 & -- & -- & $\begin{array}{r}0 \\
5 \\
22\end{array}$ & $\begin{array}{l}112 \text { SRFD } \\
112 \text { SRFD } \\
112 \text { SRFD }\end{array}$ & $\begin{array}{l}\text { CLAY } \\
\text { SAND C } \\
\text { SDGL }\end{array}$ \\
\hline MAW & 50 & 30 & -- & -- & $\begin{array}{r}3 \\
23\end{array}$ & $\begin{array}{l}112 \text { SRFD } \\
112 \text { SRFD }\end{array}$ & $\begin{array}{llll}\text { SAND C AT } & 7-10 & \text { FT } \\
\text { SDGL SAND } & \text { CLAY AND GRAVEL }\end{array}$ \\
\hline MAW & 51 & 34 & -- & 34 & $\begin{array}{r}1 \\
23 \\
30\end{array}$ & $\begin{array}{l}112 \mathrm{MRIN} \\
112 \mathrm{SRFD} \\
112 \mathrm{TILL}\end{array}$ & $\begin{array}{l}\text { CLAY SANDY } \\
\text { SDGL } \\
\text { TILL HARDPAN }\end{array}$ \\
\hline MAW & 52 & 42 & -- & 42 & $\begin{array}{r}3 \\
25\end{array}$ & $\begin{array}{l}\text { 112MRIN } \\
112 \text { SRFD }\end{array}$ & $\begin{array}{l}\text { CLAY SOET GRAY SOME SAND } \\
\text { SDGL F SAND, STREAK CLAY }\end{array}$ \\
\hline MAW & 56 & 69.5 & 69.5 & -- & $\begin{array}{l}0 \\
9.5 \\
10 \\
12 \\
40 \\
65\end{array}$ & $\begin{array}{l}112 \text { DELT } \\
112 \text { DELT } \\
112 \text { DELT } \\
112 \text { DELT } \\
112 \text { DELT } \\
112 \text { DELT }\end{array}$ & $\begin{array}{l}\text { SAND } \\
\text { STCL } \\
\text { SDGL } \\
\text { SAND F, BROWN } \\
\text { SNCL F-M, SILT, ALTERNATING LAYERS } \\
\text { SAND M-E, TRACE SILT AND CLAY }\end{array}$ \\
\hline MAW & 58 & 37 & 37 & -- & $\begin{array}{l}0 \\
17 \\
23 \\
34.5\end{array}$ & $\begin{array}{l}\text { 112SRFD } \\
112 \text { SRFD } \\
112 \text { TILL } \\
\text { BEDROCK }\end{array}$ & $\begin{array}{l}\text { GRDS WITH COBBLES } \\
\text { GRDS RUST LAYERS } \\
\text { TILL SILTY, GRAVELLY, SANDY } \\
\text { ROCK }\end{array}$ \\
\hline MAW & 59 & 41.5 & 41.5 & -- & $\begin{array}{r}0 \\
9 \\
23\end{array}$ & $\begin{array}{l}\text { 112SRFD } \\
112 \text { SRFD } \\
112 \text { SRFD }\end{array}$ & $\begin{array}{l}\text { SDGL M-C SAND, BROWN } \\
\text { SDGL M-F SAND, BROWN, TRACE SILT } \\
\text { SDST M-C SAND, TRACE GRAVEL }\end{array}$ \\
\hline MAW & 60 & 52.3 & 52.3 & -- & $\begin{array}{r}0 \\
22\end{array}$ & $\begin{array}{l}\text { 112SRFD } \\
\text { 112SRFD }\end{array}$ & $\begin{array}{l}\text { SDST M-E SAND, SILT LAYERS } \\
\text { SDST M-F SAND }\end{array}$ \\
\hline MAW & 61 & 50.2 & 50.2 & -- & $\begin{array}{l}0 \\
28 \\
41.5 \\
45.3 \\
46.1\end{array}$ & $\begin{array}{l}112 \text { SRFD } \\
112 \text { SRFD } \\
112 \text { TILI } \\
112 \text { GLCL } \\
\text { BEDROCK }\end{array}$ & $\begin{array}{l}\text { SDST M-E SAND, LAYERS SILT } \\
\text { SDGL M-F SAND, SILTY } \\
\text { TILL SAND, GRAVEL, SILT, GRAY } \\
\text { COBB GRANITIC } \\
\text { DORT NEATHERED FRACTURES }\end{array}$ \\
\hline MAW & 62 & 25 & 25 & -- & 0 & 112SRFD & GRDS M-F SAND, TRACE GRAVEL \\
\hline MAW & 63 & 15 & 15 & -- & 0 & 112SRFD & GRDS WITH COBBLES, BROWN \\
\hline MAW & 65 & 30 & 30 & 30 & $\begin{array}{r}0 \\
26\end{array}$ & $\begin{array}{l}\text { 112SRFD } \\
\text { 112TILI }\end{array}$ & $\begin{array}{l}\text { SDST F SAND } \\
\text { TILL SILTY }\end{array}$ \\
\hline MAW & 66 & 20 & 20 & -- & 0 & 112SRFD & SDST F SAND \\
\hline
\end{tabular}




\section{Table 4.--Chemical analysis of ground-water samples}

[Values are reported in microsiemens per centimeter at $25^{\circ} \mathrm{C}(\mu \mathrm{s} / \mathrm{cm})$; degrees Celsius $\left({ }^{\circ} \mathrm{C}\right)$; milligrams per liter (mg/L); micrograms per liter ( $\mu \mathrm{g} / \mathrm{L})$; TOTAL, unfiltered water; DISSOLVED, filtered water; Agency analyzing samples--U.S. Geological Survey Central Laboratories, Doraville, Georgia; --, no data collected; $<$, value less than detection limit.

\begin{tabular}{|c|c|c|c|c|c|c|c|c|c|c|}
\hline $\begin{array}{l}\text { LOCAL } \\
\text { IDENT- } \\
\text { I- } \\
\text { FIER }\end{array}$ & DATE & $\begin{array}{l}\text { TEMPER- } \\
\text { ATURE } \\
\left({ }^{\circ} \mathrm{C}\right)\end{array}$ & $\begin{array}{c}\text { TEMPER- } \\
\text { ATURE, } \\
\text { AIR } \\
\left({ }^{\circ} \mathrm{C}\right)\end{array}$ & $\begin{array}{l}\text { AGENCY } \\
\text { COL- } \\
\text { LECTING } \\
\text { SAMPIE }\end{array}$ & $\begin{array}{c}\text { AGENCY } \\
\text { ANA- } \\
\text { LYZING } \\
\text { SAMPLE }\end{array}$ & $\begin{array}{l}\text { COLOR } \\
\text { (PLAT- } \\
\text { INUM- } \\
\text { COBALT } \\
\text { UNITS) }\end{array}$ & $\begin{array}{l}\text { SPE- } \\
\text { CIFIC } \\
\text { CON- } \\
\text { DUCT- } \\
\text { ANCE } \\
(\mu \mathrm{S} / \mathrm{CM})\end{array}$ & $\begin{array}{c}\text { PH } \\
\text { (STAND- } \\
\text { ARD } \\
\text { UNITS) }\end{array}$ & $\begin{array}{c}\text { PH } \\
\text { LAB } \\
\text { (STAND- } \\
\text { ARD } \\
\text { UNITS) }\end{array}$ & $\begin{array}{l}\text { NITRO- } \\
\text { GEN, } \\
\text { AMMONIA } \\
\text { DIS- } \\
\text { SOLVED } \\
\text { (MG/L } \\
\text { AS N) }\end{array}$ \\
\hline $\begin{array}{lr}\text { BWW } & 22 \\
\text { BWW } & 26 \\
\text { BWW } & 28 \\
\text { BWW } & 30 \\
\text { CLW } & 8\end{array}$ & $\begin{array}{l}12-05-85 \\
12-06-85 \\
12-02-85 \\
12-03-85 \\
08-17-84\end{array}$ & $\begin{array}{r}9.0 \\
8.0 \\
9.0 \\
8.5 \\
12.5\end{array}$ & $\begin{array}{r}1.0 \\
.0 \\
7.0 \\
-3.5 \\
27.5\end{array}$ & $\begin{array}{l}\text { USGS } \\
\text { USGS } \\
\text { USGS } \\
\text { USGS } \\
\text { USGS }\end{array}$ & $\begin{array}{l}\text { USGS } \\
\text { USGS } \\
\text { USGS } \\
\text { USGS } \\
\text { USGS }\end{array}$ & $\begin{array}{l}3 \\
3 \\
7 \\
5 \\
8\end{array}$ & $\begin{array}{r}325 \\
148 \\
63 \\
65 \\
51\end{array}$ & $\begin{array}{l}6.4 \\
6.4 \\
6.8 \\
6.4 \\
6.6\end{array}$ & $\begin{array}{l}6.8 \\
6.9 \\
6.9 \\
7.0 \\
6.7\end{array}$ & $\begin{array}{r}0.01 \\
.01 \\
<.01 \\
<.01 \\
.04\end{array}$ \\
\hline $\begin{array}{lr}\text { CLW } & 9 \\
\text { DDW } & 44 \\
\text { DDW } & 45 \\
\text { DDW } & 46 \\
\text { DPW } & 16\end{array}$ & $\begin{array}{l}08-20-84 \\
11-06-84 \\
11-06-84 \\
11-07-84 \\
11-27-85\end{array}$ & $\begin{array}{r}9.0 \\
9.0 \\
11.5 \\
8.0 \\
8.0\end{array}$ & $\begin{array}{r}26.5 \\
12.0 \\
13.0 \\
8.5 \\
2.5\end{array}$ & $\begin{array}{l}\text { USGS } \\
\text { USGS } \\
\text { USGS } \\
\text { USGS } \\
\text { USGS }\end{array}$ & $\begin{array}{l}\text { USGS } \\
\text { USGS } \\
\text { USGS } \\
\text { USGS } \\
\text { USGS }\end{array}$ & $\begin{array}{l}1 \\
1 \\
5 \\
1 \\
3\end{array}$ & $\begin{array}{r}31 \\
220 \\
48 \\
26 \\
415\end{array}$ & $\begin{array}{l}6.2 \\
5.7 \\
6.3 \\
6.5 \\
6.7\end{array}$ & $\begin{array}{l}6.4 \\
5.8 \\
6.5 \\
6.4 \\
6.7\end{array}$ & $\begin{array}{l}<.01 \\
<.01 \\
.01 \\
.06 \\
.07\end{array}$ \\
\hline $\begin{array}{lr}\text { FMW } & 4 \\
\text { FMW } & 5 \\
\text { KTW } & 19 \\
\text { LIW } & 1 \\
\text { MAW } & 56\end{array}$ & $\begin{array}{l}08-16-84 \\
08-16-84 \\
12-06-85 \\
11-09-84 \\
11-26-85\end{array}$ & $\begin{array}{r}11.0 \\
18.0 \\
9.0 \\
9.5 \\
8.0\end{array}$ & $\begin{array}{r}29.0 \\
31.0 \\
.5 \\
8.0 \\
2.0\end{array}$ & $\begin{array}{l}\text { USGS } \\
\text { USGS } \\
\text { USGS } \\
\text { USGS } \\
\text { USGS }\end{array}$ & $\begin{array}{l}\text { USGS } \\
\text { USGS } \\
\text { USGS } \\
\text { USGS } \\
\text { USGS }\end{array}$ & $\begin{array}{r}-- \\
<1 \\
3 \\
2 \\
5\end{array}$ & $\begin{array}{r}500 \\
390 \\
89 \\
540 \\
84\end{array}$ & $\begin{array}{l}5.1 \\
5.1 \\
6.4 \\
6.4 \\
6.5\end{array}$ & $\begin{array}{l}5.3 \\
5.3 \\
7.0 \\
6.7 \\
6.8\end{array}$ & $\begin{array}{l}-- \\
-- \\
<.01 \\
<.01 \\
.01\end{array}$ \\
\hline $\begin{array}{lr}\text { MAW } & 57 \\
\text { NGS } & 1 \\
\text { NGW } & 12 \\
\text { NGW } & 13 \\
\text { NMS } & 3\end{array}$ & $\begin{array}{l}11-26-85 \\
12-03-85 \\
11-29-85 \\
12-03-85 \\
11-27-85\end{array}$ & $\begin{array}{l}9.0 \\
8.5 \\
8.0 \\
8.5 \\
9.5\end{array}$ & $\begin{array}{r}4.0 \\
-0.5 \\
2.5 \\
-2.5 \\
3.5\end{array}$ & $\begin{array}{l}\text { USGS } \\
\text { USGS } \\
\text { USGS } \\
\text { USGS } \\
\text { USGS }\end{array}$ & $\begin{array}{l}\text { USGS } \\
\text { USGS } \\
\text { USGS } \\
\text { USGS } \\
\text { USGS }\end{array}$ & $\begin{array}{l}3 \\
5 \\
5 \\
5 \\
3\end{array}$ & $\begin{array}{l}350 \\
185 \\
300 \\
187 \\
205\end{array}$ & $\begin{array}{l}5.9 \\
6.6 \\
7.3 \\
7.7 \\
6.3\end{array}$ & $\begin{array}{l}6.2 \\
7.2 \\
7.3 \\
8.1 \\
6.7\end{array}$ & $\begin{array}{l}.02 \\
.01 \\
.01 \\
.02 \\
.01\end{array}$ \\
\hline $\begin{array}{ll}\text { RBW } & 10 \\
\text { RBW } & 11 \\
\text { RBW } & 48 \\
\text { RBW } & 50 \\
\text { RBW } & 51\end{array}$ & $\begin{array}{l}08-17-84 \\
08-17-84 \\
11-07-84 \\
11-08-84 \\
11-07-84\end{array}$ & $\begin{array}{r}14.0 \\
12.0 \\
8.0 \\
11.0 \\
10.0\end{array}$ & $\begin{array}{r}29.0 \\
33.0 \\
8.0 \\
9.0 \\
8.0\end{array}$ & $\begin{array}{l}\text { USGS } \\
\text { USGS } \\
\text { USGS } \\
\text { USGS } \\
\text { USGS }\end{array}$ & $\begin{array}{l}\text { USGS } \\
\text { USGS } \\
\text { USGS } \\
\text { USGS } \\
\text { USGS }\end{array}$ & $\begin{array}{r}<1 \\
2 \\
3 \\
280 \\
21\end{array}$ & $\begin{array}{r}390 \\
54 \\
210 \\
43 \\
120\end{array}$ & $\begin{array}{l}6.0 \\
6.0 \\
5.9 \\
6.2 \\
6.2\end{array}$ & $\begin{array}{l}6.0 \\
6.2 \\
6.1 \\
5.5 \\
6.2\end{array}$ & $\begin{array}{r}.11 \\
.08 \\
<.01 \\
.68\end{array}$ \\
\hline $\begin{array}{lr}\text { RBW } & 52 \\
\text { SDW } & 3 \\
\text { SDW } & 38 \\
\text { SSW } & 10 \\
\text { SSW } & 10^{2}\end{array}$ & $\begin{array}{l}11-07-84 \\
11-09-84 \\
11-09-84 \\
12-10-85 \\
12-10-85\end{array}$ & $\begin{array}{l}8.5 \\
8.0 \\
8.5 \\
8.0 \\
9.5\end{array}$ & $\begin{array}{r}9.0 \\
14.0 \\
14.0 \\
3.5 \\
3.5\end{array}$ & $\begin{array}{l}\text { USGS } \\
\text { USGS } \\
\text { USGS } \\
\text { USGS } \\
\text { USGS }\end{array}$ & $\begin{array}{l}\text { USGS } \\
\text { USGS } \\
\text { USGS } \\
\text { USGS } \\
\text { USGS }\end{array}$ & $\begin{array}{l}2 \\
2 \\
3 \\
5 \\
3\end{array}$ & $\begin{array}{r}94 \\
92 \\
112 \\
215 \\
382\end{array}$ & $\begin{array}{l}6.2 \\
6.9 \\
6.8 \\
6.7 \\
6.8\end{array}$ & $\begin{array}{l}6.2 \\
6.8 \\
6.9 \\
7.0 \\
7.2\end{array}$ & $\begin{array}{r}.22 \\
.08 \\
.05 \\
<.01 \\
.01\end{array}$ \\
\hline
\end{tabular}


Table 4.--Chemical analysis of ground-water samples--Continued

\begin{tabular}{|c|c|c|c|c|c|c|c|c|c|c|}
\hline $\begin{array}{l}\text { LOCAL } \\
\text { IDENT- } \\
\text { I- } \\
\text { FIER }\end{array}$ & DATE & $\begin{array}{l}\text { CARBON, } \\
\text { ORGANIC } \\
\text { DIS- } \\
\text { SOLVED } \\
\text { (MG/L } \\
\text { AS C) }\end{array}$ & $\begin{array}{l}\text { CALCIUM } \\
\text { DIS- } \\
\text { SOLVED } \\
\text { (MG/L } \\
\text { AS CA) }\end{array}$ & $\begin{array}{l}\text { MAGNE- } \\
\text { SIUM, } \\
\text { DIS- } \\
\text { SOLVED } \\
\text { (MG/L } \\
\text { AS MG) }\end{array}$ & $\begin{array}{l}\text { SODIUM, } \\
\text { DIS- } \\
\text { SOLVED } \\
\text { (MG/L } \\
\text { AS NA) }\end{array}$ & $\begin{array}{l}\text { POTAS- } \\
\text { SIUM, } \\
\text { DIS- } \\
\text { SOLVED } \\
\text { (MG/L } \\
\text { AS K) }\end{array}$ & $\begin{array}{l}\text { CHLO- } \\
\text { RIDE, } \\
\text { DIS- } \\
\text { SOLVED } \\
\text { (MG/L } \\
\text { AS CL) }\end{array}$ & $\begin{array}{l}\text { SULFATE } \\
\text { DIS- } \\
\text { SOLVED } \\
(\mathrm{MG} / \mathrm{L} \\
\left.\text { AS } \mathrm{SO}_{4}\right)\end{array}$ & $\begin{array}{l}\text { FLUO- } \\
\text { RIDE, } \\
\text { DIS- } \\
\text { SOLVED } \\
\text { (MG/L } \\
\text { AS F) }\end{array}$ & $\begin{array}{l}\text { SILICA, } \\
\text { DIS- } \\
\text { SOLVED } \\
\text { (MG/L } \\
\text { AS } \\
\mathrm{SIO}_{2} \text { ) }\end{array}$ \\
\hline $\begin{array}{lr}\text { BWW } & 22 \\
\text { BWW } & 26 \\
\text { BWW } & 28 \\
\text { BWW } & 30 \\
\text { CLW } & 8\end{array}$ & $\begin{array}{l}12-05-85 \\
12-06-85 \\
12-02-85 \\
12-03-85 \\
08-17-84\end{array}$ & $\begin{array}{r}0.8 \\
1.7 \\
1.4 \\
2.3 \\
.4\end{array}$ & $\begin{array}{r}16 \\
13 \\
4.5 \\
6.1 \\
4.1\end{array}$ & $\begin{array}{l}4.1 \\
2.7 \\
1.1 \\
1.2 \\
.38\end{array}$ & $\begin{array}{l}30 \\
6.6 \\
3.2 \\
3.8 \\
2.4\end{array}$ & $\begin{array}{l}5.7 \\
2.9 \\
3.6 \\
1.3 \\
2.3\end{array}$ & $\begin{array}{l}64 \\
10 \\
3.6 \\
3.4 \\
2.4\end{array}$ & $\begin{array}{r}11 \\
12 \\
7.3 \\
4.7 \\
6.0\end{array}$ & $\begin{array}{l}<0.1 \\
<.1 \\
<.1 \\
<.1 \\
<.1\end{array}$ & $\begin{array}{c}15 \\
21 \\
14 \\
19 \\
9.8\end{array}$ \\
\hline $\begin{array}{lr}\text { CLW } & 9 \\
\text { DDW } & 44 \\
\text { DDW } & 45 \\
\text { DDW } & 46 \\
\text { DPW } & 16\end{array}$ & $\begin{array}{l}08-20-84 \\
11-06-84 \\
11-06-84 \\
11-07-84 \\
11-27-85\end{array}$ & $\begin{array}{r}.6 \\
.3 \\
.5 \\
2.8 \\
.9\end{array}$ & $\begin{array}{l}2.5 \\
4.3 \\
3.6 \\
1.8 \\
28\end{array}$ & $\begin{array}{l}.49 \\
.91 \\
.95 \\
.42 \\
5.4\end{array}$ & $\begin{array}{c}1.6 \\
34 \\
3.2 \\
1.9 \\
39\end{array}$ & $\begin{array}{r}.9 \\
1.9 \\
1.0 \\
.6 \\
5.6\end{array}$ & $\begin{array}{l}1.5 \\
48 \\
1.7 \\
2.1 \\
62\end{array}$ & $\begin{array}{l}5.4 \\
2.8 \\
6.6 \\
2.4 \\
16\end{array}$ & $\begin{array}{l}.1 \\
<.1 \\
<.1 \\
<.1 \\
<.1\end{array}$ & $\begin{array}{l}8.7 \\
10 \\
12 \\
9.3 \\
18\end{array}$ \\
\hline $\begin{array}{lr}\text { FMW } & 4 \\
\text { FMW } & 5 \\
\text { KTW } & 19 \\
\text { LIW } & 1 \\
\text { MAW } & 56\end{array}$ & $\begin{array}{l}08-16-84 \\
08-16-84 \\
12-06-85 \\
11-09-84 \\
11-26-85\end{array}$ & $\begin{array}{l}-- \\
-- \\
.7 \\
2.9 \\
1.2\end{array}$ & $\begin{array}{r}12 \\
5.1 \\
7.2 \\
11 \\
6.3\end{array}$ & $\begin{array}{l}3.4 \\
1.1 \\
1.6 \\
1.4 \\
2.0\end{array}$ & $\begin{array}{c}65 \\
55 \\
3.8 \\
79 \\
4.2\end{array}$ & $\begin{array}{l}-- \\
2.6 \\
1.8 \\
2.4 \\
2.1\end{array}$ & $\begin{array}{c}-- \\
94 \\
3.3 \\
120 \\
2.8\end{array}$ & $\begin{array}{l}-- \\
18 \\
10 \\
9.5 \\
16\end{array}$ & $\begin{array}{r}-- \\
<.1 \\
<.1 \\
<.1 \\
.1\end{array}$ & $\begin{array}{l}11 \\
9.7 \\
20 \\
7.2 \\
20^{\circ}\end{array}$ \\
\hline $\begin{array}{lr}\text { MAW } & 57 \\
\text { NGS } & 1 \\
\text { NGW } & 12 \\
\text { NGW } & 13 \\
\text { NMS } & 3\end{array}$ & $\begin{array}{l}11-26-85 \\
12-03-85 \\
11-29-85 \\
12-03-85 \\
11-27-85\end{array}$ & $\begin{array}{r}2.2 \\
1.0 \\
.9 \\
1.0 \\
.8\end{array}$ & $\begin{array}{l}36 \\
12 \\
15 \\
16 \\
13\end{array}$ & $\begin{array}{l}7.7 \\
2.2 \\
2.9 \\
1.9 \\
2.4\end{array}$ & $\begin{array}{l}13 \\
16 \\
35 \\
15 \\
17\end{array}$ & $\begin{array}{l}3.3 \\
3.4 \\
5.8 \\
3.7 \\
3.5\end{array}$ & $\begin{array}{l}6.0 \\
26 \\
51 \\
6.0 \\
24\end{array}$ & $\begin{array}{c}79 \\
9.7 \\
11 \\
15 \\
11\end{array}$ & $\begin{array}{r}<.1 \\
<.1 \\
.3 \\
.2 \\
<.1\end{array}$ & $\begin{array}{l}16 \\
11 \\
10 \\
14 \\
15\end{array}$ \\
\hline $\begin{array}{lr}\text { RBW } & 52 \\
\text { SDW } & 3 \\
\text { SDW } & 38 \\
\text { SSW } & 10 \\
\text { SSW } & 10\end{array}$ & $\begin{array}{l}11-07-84 \\
11-09-84 \\
11-09-84 \\
12-10-85 \\
12-10-85\end{array}$ & $\begin{array}{r}.4 \\
.9 \\
1.8 \\
2.5 \\
1.5\end{array}$ & $\begin{array}{l}4.4 \\
2.1 \\
9.8 \\
21 \\
33\end{array}$ & $\begin{array}{l}1.3 \\
.36 \\
2.0 \\
5.8 \\
7.2\end{array}$ & $\begin{array}{c}6.9 \\
13 \\
4.4 \\
10 \\
20\end{array}$ & $\begin{array}{l}1.6 \\
1.6 \\
4.2 \\
2.3 \\
3.5\end{array}$ & $\begin{array}{r}6.8 \\
8.7 \\
6.2 \\
6.8 \\
62\end{array}$ & $\begin{array}{l}8.7 \\
9.3 \\
7.2 \\
14 \\
16\end{array}$ & $\begin{array}{r}<.1 \\
<.1 \\
<.1 \\
.1 \\
.1\end{array}$ & $\begin{array}{l}9.5 \\
8.0 \\
16 \\
17 \\
12\end{array}$ \\
\hline
\end{tabular}


Table 4.--Chemical analysis of ground-water samples--Continued

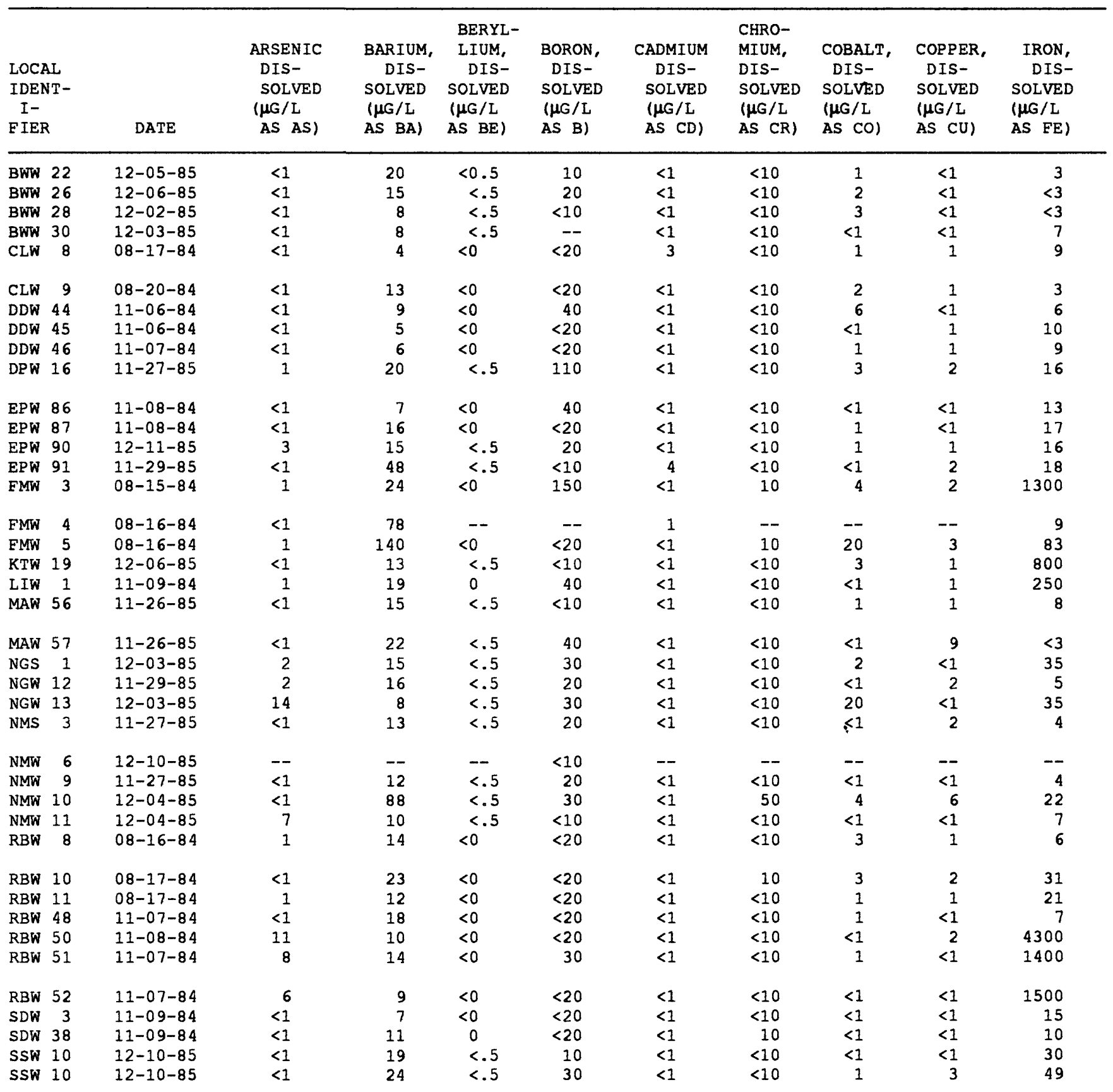


Table 4.--Chemical analysis of ground-water samples--Continued

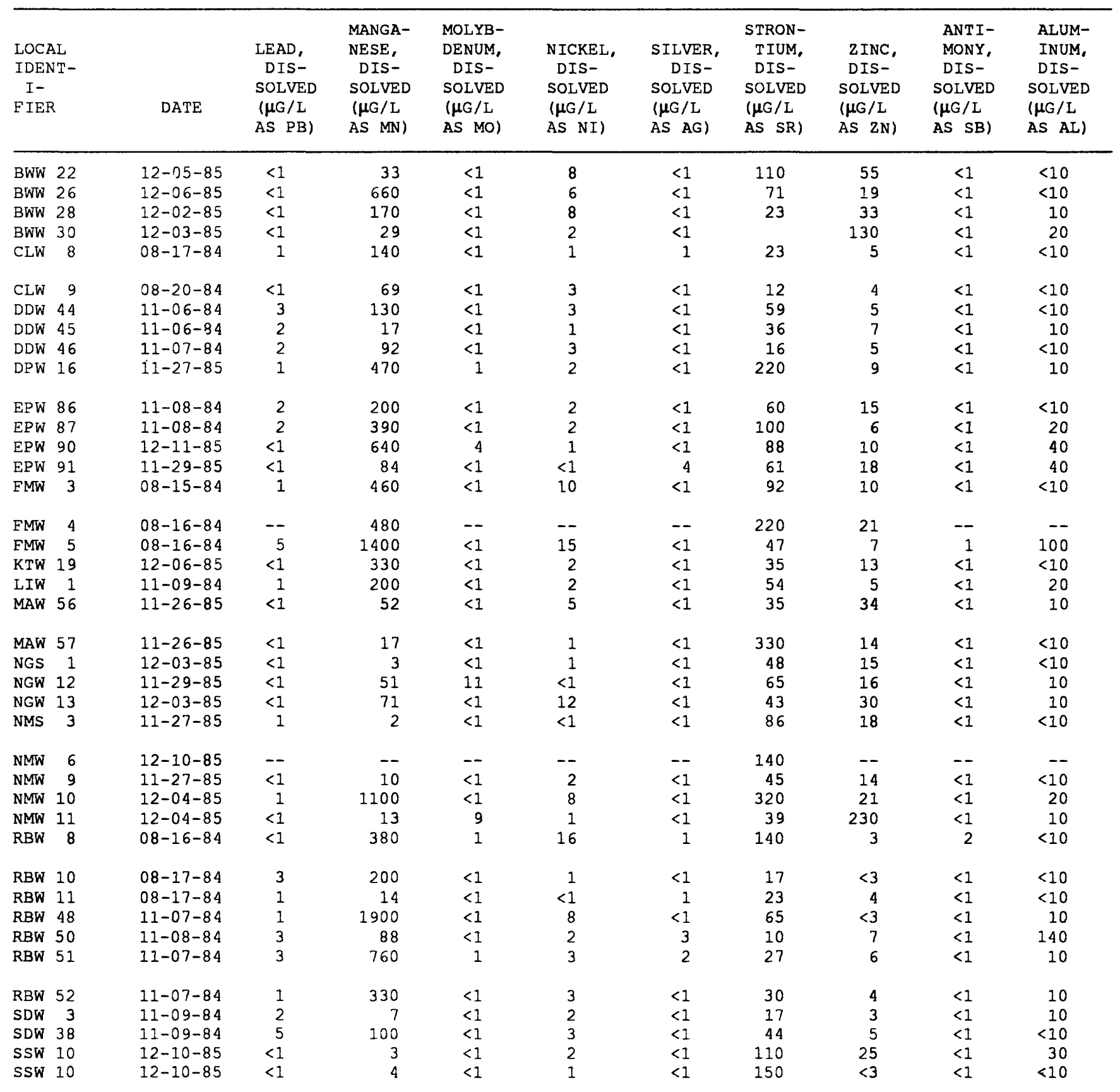


Table 4.--Chemical analysis of ground-water samples--Continued

\begin{tabular}{|c|c|c|c|c|c|c|c|c|c|c|}
\hline $\begin{array}{l}\text { LOCAL } \\
\text { IDENT- } \\
\text { I- } \\
\text { FIER }\end{array}$ & DATE & $\begin{array}{l}\text { LITHIUM } \\
\text { DIS- } \\
\text { SOLVED } \\
\text { ( } \mu \text { G/L } \\
\text { AS LI) }\end{array}$ & $\begin{array}{l}\text { SELE- } \\
\text { NIUM, } \\
\text { DIS- } \\
\text { SOLVED } \\
\text { ( } \mu G / L \\
\text { AS SE) }\end{array}$ & $\begin{array}{c}\text { DI- } \\
\text { CHLORO- } \\
\text { BROMO- } \\
\text { METHANE } \\
\text { TOTAL } \\
\text { ( } \text { LG/L) }\end{array}$ & $\begin{array}{l}\text { CARBON- } \\
\text { TETRA- } \\
\text { CHLO- } \\
\text { RIDE } \\
\text { TOTAL } \\
(\mu G / L)\end{array}$ & $\begin{array}{l}1,2-D I- \\
\text { CHLORO- } \\
\text { ETHANE } \\
\text { TOTAL } \\
(\mu G / L)\end{array}$ & $\begin{array}{l}\text { BROM- } \\
\text { OFORM } \\
\text { TOTAL } \\
(\mu G / L)\end{array}$ & $\begin{array}{c}\text { CHLORO- } \\
\text { DI- } \\
\text { BROMO- } \\
\text { METHANE } \\
\text { TOTAL } \\
(\mu G / L)\end{array}$ & $\begin{array}{l}\text { CHLORO- } \\
\text { FORM } \\
\text { TOTAL } \\
(\mu \mathrm{G} / \mathrm{L})\end{array}$ & $\begin{array}{c}\text { TOLUENE } \\
\text { TOTAL } \\
(\mu \mathrm{G} / \mathrm{L})\end{array}$ \\
\hline $\begin{array}{lr}\text { BWW } & 22 \\
\text { BWW } & 26 \\
\text { BWW } & 28 \\
\text { BWW } & 30 \\
\text { CLW } & 8\end{array}$ & $\begin{array}{l}12-05-85 \\
12-06-85 \\
12-02-85 \\
12-03-85 \\
08-17-84\end{array}$ & $\begin{array}{r}15 \\
7 \\
5 \\
<4 \\
<4\end{array}$ & $\begin{array}{l}<1 \\
<1 \\
<1 \\
<1 \\
<1\end{array}$ & $\begin{array}{l}<3.0 \\
<3.0 \\
<3.0 \\
<3.0 \\
<3.0\end{array}$ & $\begin{array}{l}<3.0 \\
<3.0 \\
<3.0 \\
<3.0 \\
<3.0\end{array}$ & $\begin{array}{l}<3.0 \\
<3.0 \\
<3.0 \\
<3.0 \\
<3.0\end{array}$ & $\begin{array}{l}<3.0 \\
<3.0 \\
<3.0 \\
<3.0 \\
<3.0\end{array}$ & $\begin{array}{l}<3.0 \\
<3.0 \\
<3.0 \\
<3.0 \\
<3.0\end{array}$ & $\begin{array}{l}<3.0 \\
<3.0 \\
<3.0 \\
<3.0 \\
<3.0\end{array}$ & $\begin{array}{l}<3.0 \\
<3.0 \\
<3.0 \\
<3.0 \\
<3.0\end{array}$ \\
\hline $\begin{array}{lr}\text { CLW } & 9 \\
\text { DDW } & 44 \\
\text { DDW } & 45 \\
\text { DDW } & 46 \\
\text { DPW } & 16\end{array}$ & $\begin{array}{l}08-20-84 \\
11-06-84 \\
11-06-84 \\
11-07-84 \\
11-27-85\end{array}$ & $\begin{array}{r}<4 \\
<4 \\
<4 \\
<4 \\
7\end{array}$ & $\begin{array}{l}<1 \\
<1 \\
<1 \\
<1 \\
<1\end{array}$ & $\begin{array}{l}<3.0 \\
<3.0 \\
<3.0 \\
<3.0 \\
<3.0\end{array}$ & $\begin{array}{l}<3.0 \\
<3.0 \\
<3.0 \\
<3.0 \\
<3.0\end{array}$ & $\begin{array}{l}<3.0 \\
<3.0 \\
<3.0 \\
<3.0 \\
<3.0\end{array}$ & $\begin{array}{l}<3.0 \\
<3.0 \\
<3.0 \\
<3.0 \\
<3.0\end{array}$ & $\begin{array}{l}<3.0 \\
<3.0 \\
<3.0 \\
<3.0 \\
<3.0\end{array}$ & $\begin{array}{l}<3.0 \\
<3.0 \\
<3.0 \\
<3.0 \\
<3.0\end{array}$ & $\begin{array}{l}<3.0 \\
<3.0 \\
<3.0 \\
<3.0 \\
<3.0\end{array}$ \\
\hline $\begin{array}{lr}\text { EPW } & 86 \\
\text { EPW } & 87 \\
\text { EPW } & 90 \\
\text { EPW } & 91 \\
\text { EMW } & 3\end{array}$ & $\begin{array}{l}11-08-84 \\
11-08-84 \\
12-11-85 \\
11-29-85 \\
08-15-84\end{array}$ & $\begin{array}{r}<4 \\
<4 \\
<4 \\
<4 \\
4\end{array}$ & $\begin{array}{r}<1 \\
<1 \\
<1 \\
1 \\
<1\end{array}$ & $\begin{array}{l}<3.0 \\
<3.0 \\
<3.0 \\
<3.0 \\
<3.0\end{array}$ & $\begin{array}{l}<3.0 \\
<3.0 \\
<3.0 \\
<3.0 \\
<3.0\end{array}$ & $\begin{array}{l}<3.0 \\
<3.0 \\
<3.0 \\
<3.0 \\
<3.0\end{array}$ & $\begin{array}{l}<3.0 \\
<3.0 \\
<3.0 \\
<3.0 \\
<3.0\end{array}$ & $\begin{array}{l}<3.0 \\
<3.0 \\
<3.0 \\
<3.0 \\
<3.0\end{array}$ & $\begin{array}{l}<3.0 \\
<3.0 \\
<3.0 \\
<3.0 \\
<3.0\end{array}$ & $\begin{array}{l}<3.0 \\
<3.0 \\
<3.0 \\
<3.0 \\
<3.0\end{array}$ \\
\hline $\begin{array}{lr}\text { FMW } & 4 \\
\text { EMW } & 5 \\
\text { KTW } & 19 \\
\text { LIW } & 1 \\
\text { MAW } & 56\end{array}$ & $\begin{array}{l}08-16-84 \\
08-16-84 \\
12-06-85 \\
11-09-84 \\
11-26-85\end{array}$ & $\begin{array}{l}<4 \\
<4 \\
<4 \\
<4 \\
10\end{array}$ & $\begin{array}{l}-- \\
<1 \\
<1 \\
<1 \\
<1\end{array}$ & $\begin{array}{l}<3.0 \\
<3.0 \\
<3.0 \\
<3.0 \\
<3.0\end{array}$ & $\begin{array}{l}<3.0 \\
<3.0 \\
<3.0 \\
<3.0 \\
<3.0\end{array}$ & $\begin{array}{l}<3.0 \\
<3.0 \\
<3.0 \\
<3.0 \\
<3.0\end{array}$ & $\begin{array}{l}<3.0 \\
<3.0 \\
<3.0 \\
<3.0 \\
<3.0\end{array}$ & $\begin{array}{l}<3.0 \\
<3.0 \\
<3.0 \\
<3.0 \\
<3.0\end{array}$ & $\begin{array}{l}<3.0 \\
<3.0 \\
<3.0 \\
<3.0 \\
<3.0\end{array}$ & $\begin{array}{l}<3.0 \\
<3.0 \\
<3.0 \\
<3.0 \\
<3.0\end{array}$ \\
\hline $\begin{array}{lr}\text { MAW } & 57 \\
\text { NGS } & 1 \\
\text { NGW } & 12 \\
\text { NGW } & 13 \\
\text { NMS } & 3\end{array}$ & $\begin{array}{l}11-26-85 \\
12-03-85 \\
11-29-85 \\
12-03-85 \\
11-27-85\end{array}$ & $\begin{array}{r}10 \\
5 \\
5 \\
7 \\
<4\end{array}$ & $\begin{array}{l}<1 \\
<1 \\
<1 \\
<1 \\
<1\end{array}$ & $\begin{array}{l}<3.0 \\
<3.0 \\
<3.0 \\
<3.0 \\
<3.0\end{array}$ & $\begin{array}{l}<3.0 \\
<3.0 \\
<3.0 \\
<3.0 \\
<3.0\end{array}$ & $\begin{array}{l}<3.0 \\
<3.0 \\
<3.0 \\
<3.0 \\
<3.0\end{array}$ & $\begin{array}{l}<3.0 \\
<3.0 \\
<3.0 \\
<3.0 \\
<3.0\end{array}$ & $\begin{array}{l}<3.0 \\
<3.0 \\
<3.0 \\
<3.0 \\
<3.0\end{array}$ & $\begin{array}{l}<3.0 \\
<3.0 \\
<3.0 \\
<3.0 \\
<3.0\end{array}$ & $\begin{array}{l}<3.0 \\
<3.0 \\
<3.0 \\
<3.0 \\
<3.0\end{array}$ \\
\hline $\begin{array}{lr}\text { NMW } & 6 \\
\text { NMW } & 9 \\
\text { NMW } & 10 \\
\text { NMW } & 11 \\
\text { RBW } & 8\end{array}$ & $\begin{array}{l}12-10-85 \\
11-27-85 \\
12-04-85 \\
12-04-85 \\
08-16-84\end{array}$ & $\begin{array}{r}-- \\
<4 \\
19 \\
4 \\
<4\end{array}$ & $\begin{array}{l}-- \\
<1 \\
<1 \\
<1 \\
<1\end{array}$ & $\begin{array}{l}<3.0 \\
<3.0 \\
<3.0 \\
<3.0 \\
<3.0\end{array}$ & $\begin{array}{l}<3.0 \\
<3.0 \\
<3.0 \\
<3.0 \\
<3.0\end{array}$ & $\begin{array}{l}<3.0 \\
<3.0 \\
<3.0 \\
<3.0 \\
<3.0\end{array}$ & $\begin{array}{l}<3.0 \\
<3.0 \\
<3.0 \\
<3.0 \\
<3.0\end{array}$ & $\begin{array}{l}<3.0 \\
<3.0 \\
<3.0 \\
<3.0 \\
<3.0\end{array}$ & $\begin{array}{l}<3.0 \\
<3.0 \\
<3.0 \\
<3.0 \\
<3.0\end{array}$ & $\begin{array}{l}<3.0 \\
<3.0 \\
<3.0 \\
<3.0 \\
<3.0\end{array}$ \\
\hline $\begin{array}{ll}\text { RBW } & 10 \\
\text { RBW } & 11 \\
\text { RBW } & 48 \\
\text { RBW } & 50 \\
\text { RBW } & 51\end{array}$ & $\begin{array}{l}11-08-84 \\
08-17-84 \\
11-07-84 \\
11-08-84 \\
11-07-84\end{array}$ & $\begin{array}{l}<4 \\
<4 \\
<4 \\
<4 \\
<4\end{array}$ & $\begin{array}{l}<1 \\
<1 \\
<1 \\
<1 \\
<1\end{array}$ & $\begin{array}{l}<3.0 \\
<3.0 \\
<3.0 \\
<3.0 \\
<3.0\end{array}$ & $\begin{array}{l}<3.0 \\
<3.0 \\
<3.0 \\
<3.0 \\
<3.0\end{array}$ & $\begin{array}{l}<3.0 \\
<3.0 \\
<3.0 \\
<3.0 \\
<3.0\end{array}$ & $\begin{array}{l}<3.0 \\
<3.0 \\
<3.0 \\
<3.0 \\
<3.0\end{array}$ & $\begin{array}{l}<3.0 \\
<3.0 \\
<3.0 \\
<3.0 \\
<3.0\end{array}$ & $\begin{array}{l}<3.0 \\
<3.0 \\
<3.0 \\
<3.0 \\
<3.0\end{array}$ & $\begin{array}{l}<3.0 \\
<3.0 \\
<3.0 \\
<3.0 \\
<3.0\end{array}$ \\
\hline $\begin{array}{lr}\text { RBW } & 52 \\
\text { SDW } & 3 \\
\text { SDW } & 38 \\
\text { SSW } & 10 \\
\text { SSW } & 10\end{array}$ & $\begin{array}{l}11-07-84 \\
11-09-84 \\
11-09-84 \\
12-10-85 \\
12-10-85\end{array}$ & $\begin{array}{r}<4 \\
<4 \\
4 \\
10 \\
6\end{array}$ & $\begin{array}{r}<1 \\
<1 \\
<1 \\
1 \\
<1\end{array}$ & $\begin{array}{l}<3.0 \\
<3.0 \\
<3.0 \\
<3.0 \\
<3.0\end{array}$ & $\begin{array}{l}<3.0 \\
<3.0 \\
<3.0 \\
<3.0 \\
<3.0\end{array}$ & $\begin{array}{l}<3.0 \\
<3.0 \\
<3.0 \\
<3.0 \\
<3.0\end{array}$ & $\begin{array}{l}<3.0 \\
<3.0 \\
<3.0 \\
<3.0 \\
<3.0\end{array}$ & $\begin{array}{l}<3.0 \\
<3.0 \\
<3.0 \\
<3.0 \\
<3.0\end{array}$ & $\begin{array}{l}<3.0 \\
<3.0 \\
<3.0 \\
<3.0 \\
<3.0\end{array}$ & $\begin{array}{l}<3.0 \\
<3.0 \\
<3.0 \\
<3.0 \\
<3.0\end{array}$ \\
\hline
\end{tabular}


Table 4.--Chemical analysis of ground-water samples--Continued

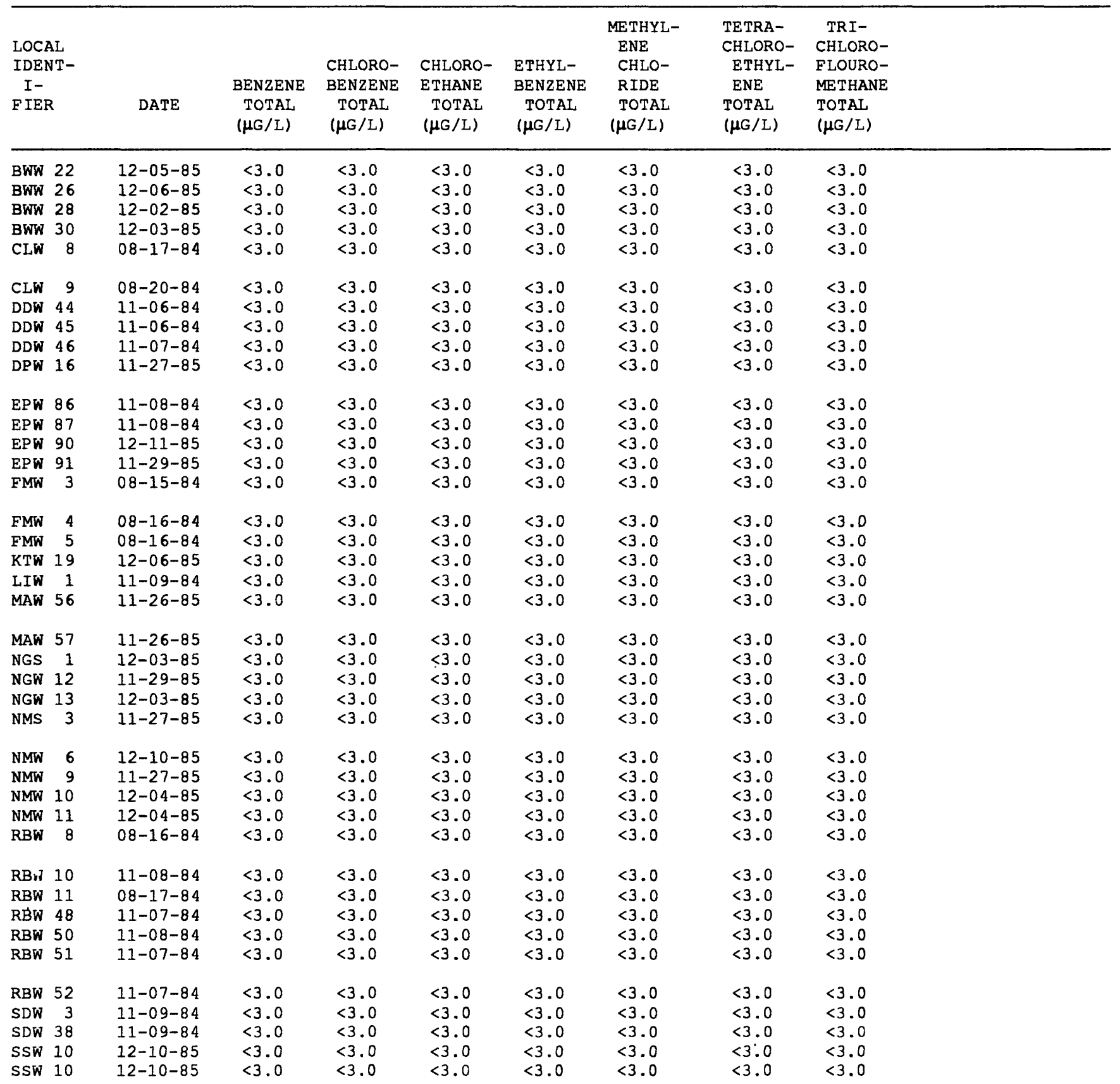


Table 4.--Chemical analysis of ground-water samples--Continued

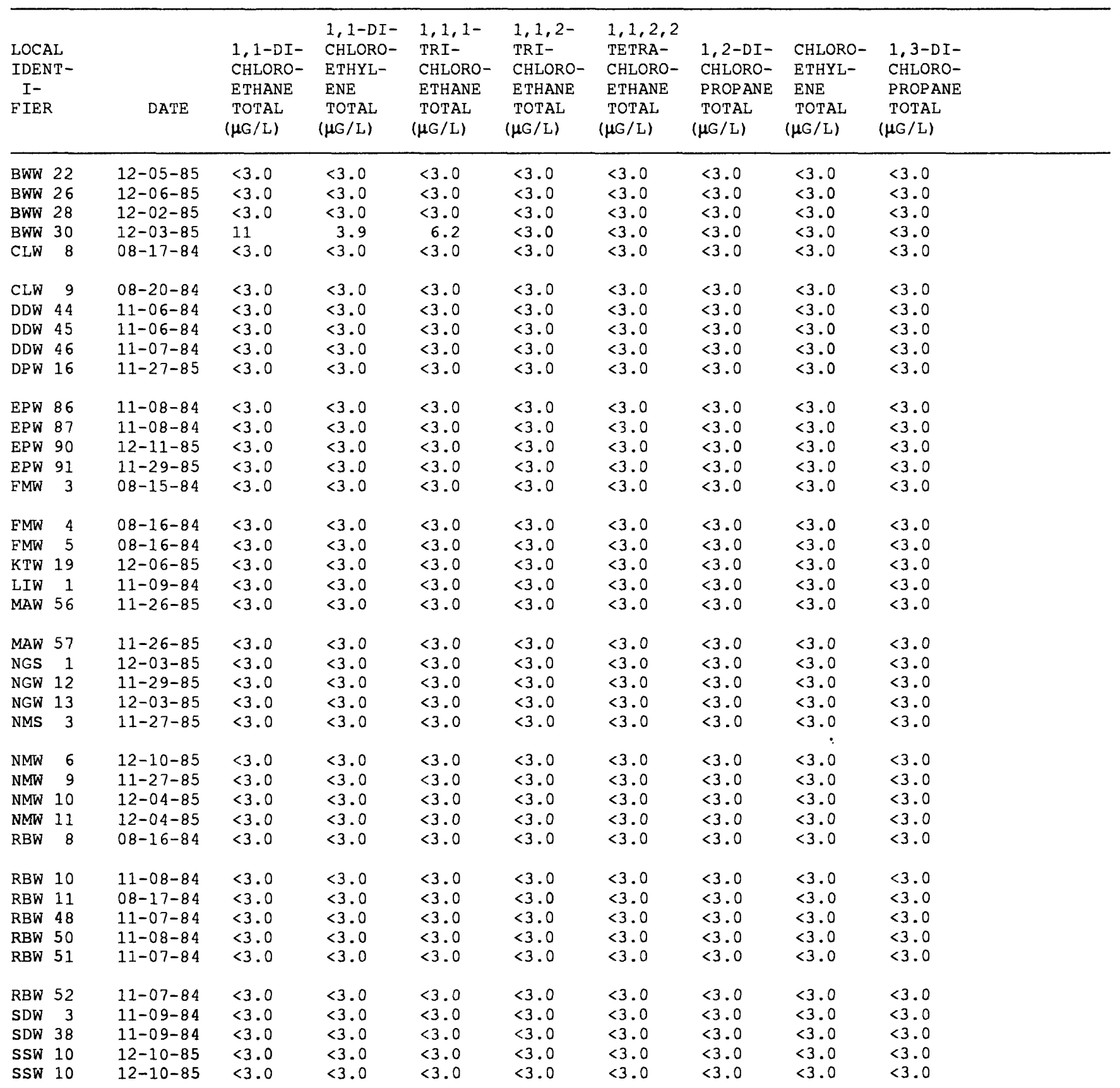


Table 4.--Chemical analysis of ground-water samples--Continued

\begin{tabular}{|c|c|c|c|c|c|c|c|c|}
\hline $\begin{array}{l}\text { LOCAL } \\
\text { IDENT- } \\
\text { I- } \\
\text { FIER }\end{array}$ & DATE & $\begin{array}{l}2- \\
\text { CHLORO- } \\
\text { ETHYL- } \\
\text { VINYL- } \\
\text { ETHER } \\
\text { TOTAL } \\
(\mu G / L)\end{array}$ & \begin{tabular}{l}
\multicolumn{1}{c}{ DI- } \\
CHLORO- \\
DI- \\
FLUORO- \\
METHANE \\
TOTAL \\
$(\mu G / L)$
\end{tabular} & $\begin{array}{l}\text { VINYL } \\
\text { CHLO- } \\
\text { RIDE } \\
\text { TOTAL } \\
(\mu \mathrm{G} / \mathrm{L})\end{array}$ & $\begin{array}{c}\text { TRI- } \\
\text { CHLORO- } \\
\text { ETHYL- } \\
\text { ENE } \\
\text { TOTAL } \\
(\mu \mathrm{G} / \mathrm{L})\end{array}$ & $\begin{array}{c}\text { MERCURY } \\
\text { DIS- } \\
\text { SOLVED } \\
\text { ( } \mu \mathrm{G} / \mathrm{L} \\
\text { AS HG) }\end{array}$ & $\begin{array}{c}\text { SPE- } \\
\text { CIFIC } \\
\text { CON- } \\
\text { DUCT- } \\
\text { ANCE } \\
\text { LAB } \\
(\mu \mathrm{S} / \mathrm{CM})\end{array}$ & $\begin{array}{c}\text { ALKA- } \\
\text { LINITY } \\
\text { LAB } \\
\text { (MG/L } \\
\text { AS } \\
\text { CACO3) }\end{array}$ \\
\hline $\begin{array}{lr}\text { CLW } & 9 \\
\text { DDW } & 44 \\
\text { DDW } & 45 \\
\text { DDW } & 46 \\
\text { DPW } & 16\end{array}$ & $\begin{array}{l}08-20-84 \\
11-06-84 \\
11-06-84 \\
11-07-84 \\
11-27-85\end{array}$ & $\begin{array}{l}<3.0 \\
<3.0 \\
<3.0 \\
<3.0 \\
<3.0\end{array}$ & $\begin{array}{l}<3.0 \\
<3.0 \\
<3.0 \\
<3.0 \\
<3.0\end{array}$ & $\begin{array}{l}<3.0 \\
<3.0 \\
<3.0 \\
<3.0 \\
<3.0\end{array}$ & $\begin{array}{l}<3.0 \\
<3.0 \\
<3.0 \\
<3.0 \\
<3.0\end{array}$ & $\begin{array}{l}<.1 \\
<.1 \\
<.1 \\
<.1 \\
.2\end{array}$ & $\begin{array}{r}31 \\
210 \\
46 \\
25 \\
414\end{array}$ & $\begin{array}{l}7.0 \\
18 \\
13 \\
8.0 \\
48\end{array}$ \\
\hline $\begin{array}{lr}\text { FMW } & 4 \\
\text { FMW } & 5 \\
\text { KTW } & 19 \\
\text { LIW } & 1 \\
\text { MAW } & 56\end{array}$ & $\begin{array}{l}08-16-84 \\
08-16-84 \\
12-06-85 \\
11-09-84 \\
11-26-85\end{array}$ & $\begin{array}{l}<3.0 \\
<3.0 \\
<3.0 \\
<3.0 \\
<3.0\end{array}$ & $\begin{array}{l}<3.0 \\
<3.0 \\
<3.0 \\
<3.0 \\
<3.0\end{array}$ & $\begin{array}{l}<3.0 \\
<3.0 \\
<3.0 \\
<3.0 \\
<3.0\end{array}$ & $\begin{array}{l}<3.0 \\
<3.0 \\
<3.0 \\
<3.0 \\
<3.0\end{array}$ & $\begin{array}{l}-- \\
.3 \\
<.1 \\
<.1 \\
<.1\end{array}$ & $\begin{array}{r}459 \\
347 \\
91 \\
493 \\
85\end{array}$ & $\begin{array}{l}-- \\
11 \\
10 \\
35 \\
14\end{array}$ \\
\hline $\begin{array}{lr}\text { MAW } & 57 \\
\text { NGS } & 1 \\
\text { NGW } & 12 \\
\text { NGW } & 13 \\
\text { NMS } & 3\end{array}$ & $\begin{array}{l}11-26-85 \\
12-03-85 \\
11-29-85 \\
12-03-85 \\
11-27-85\end{array}$ & $\begin{array}{l}<3.0 \\
<3.0 \\
<3.0 \\
<3.0 \\
<3.0\end{array}$ & $\begin{array}{l}<3.0 \\
<3.0 \\
<3.0 \\
<3.0 \\
<3.0\end{array}$ & $\begin{array}{l}<3.0 \\
<3.0 \\
<3.0 \\
<3.0 \\
<3.0\end{array}$ & $\begin{array}{l}<3.0 \\
<3.0 \\
<3.0 \\
<3.0 \\
<3.0\end{array}$ & $\begin{array}{l}<.1 \\
<.1 \\
<.1 \\
<.1 \\
.4\end{array}$ & $\begin{array}{l}342 \\
181 \\
297 \\
185 \\
207\end{array}$ & $\begin{array}{l}12 \\
29 \\
38 \\
58 \\
19\end{array}$ \\
\hline $\begin{array}{ll}\text { RBW } & 10 \\
\text { RBW } & 11 \\
\text { RBW } & 48 \\
\text { RBW } & 50\end{array}$ & $\begin{array}{l}08-17-84 \\
11-08-84 \\
08-17-84 \\
11-07-84 \\
11-08-84\end{array}$ & $\begin{array}{l}<-- \\
<3.0 \\
<3.0 \\
<3.0 \\
<3.0\end{array}$ & $\begin{array}{l}<3.0 \\
<3.0 \\
<3.0 \\
<3.0\end{array}$ & $\begin{array}{l}-- \\
<3.0 \\
<3.0 \\
<3.0 \\
<3.0\end{array}$ & $\begin{array}{l}<-- \\
<3.0 \\
<3.0 \\
<3.0 \\
<3.0\end{array}$ & $\begin{array}{l}.2 \\
-- \\
<.1 \\
<.1 \\
<.1\end{array}$ & $\begin{array}{r}375 \\
-- \\
53 \\
104 \\
26\end{array}$ & $\begin{array}{c}6.0 \\
-- \\
13 \\
26 \\
5.0\end{array}$ \\
\hline $\begin{array}{lr}\text { RBW } & 51 \\
\text { RBW } & 52 \\
\text { SDW } & 3 \\
\text { SDW } & 38 \\
\text { SSW } & 10 \\
\text { SSW } & 10\end{array}$ & $\begin{array}{l}11-07-84 \\
11-07-84 \\
11-09-84 \\
11-09-84 \\
12-10-85 \\
12-10-85\end{array}$ & $\begin{array}{l}<3.0 \\
<3.0 \\
<3.0 \\
<3.0 \\
<3.0 \\
<3.0\end{array}$ & $\begin{array}{l}<3.0 \\
<3.0 \\
<3.0 \\
<3.0 \\
<3.0 \\
<3.0\end{array}$ & $\begin{array}{l}<3.0 \\
<3.0 \\
<3.0 \\
<3.0 \\
<3.0 \\
<3.0\end{array}$ & $\begin{array}{l}<3.0 \\
<3.0 \\
<3.0 \\
<3.0 \\
<3.0 \\
<3.0\end{array}$ & $\begin{array}{l}<.1 \\
<.1 \\
<.1 \\
<.1 \\
<.1 \\
<.1\end{array}$ & $\begin{array}{r}119 \\
78 \\
78 \\
99 \\
210 \\
369\end{array}$ & $\begin{array}{l}20 \\
15 \\
17 \\
32 \\
70 \\
50\end{array}$ \\
\hline
\end{tabular}


Table 4.--Chemical analysis of ground-water samples--Continued

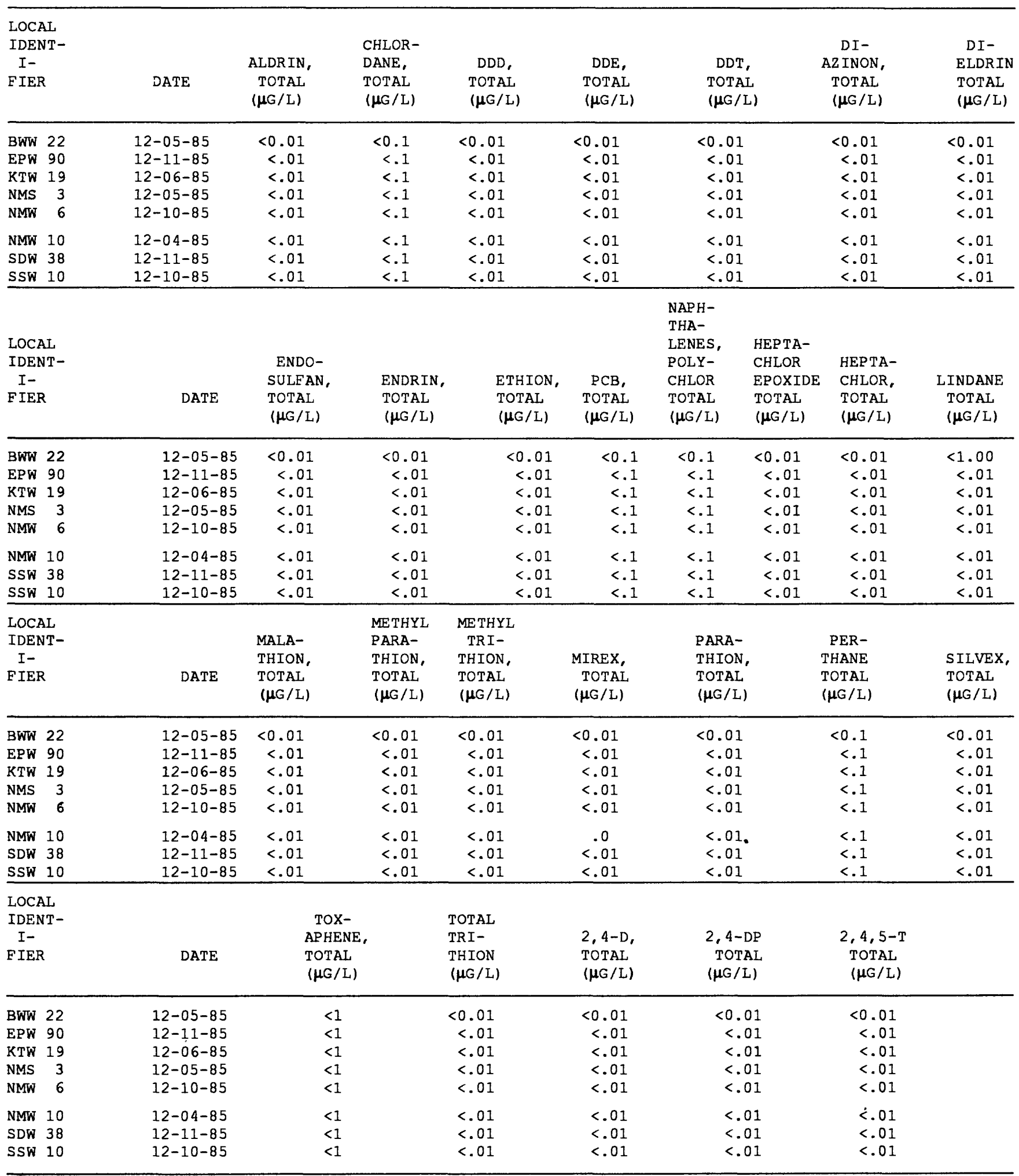

\footnotetext{
$1_{A T}$ WELL

${ }^{2}$ AT TREATMENT PLANT
} 


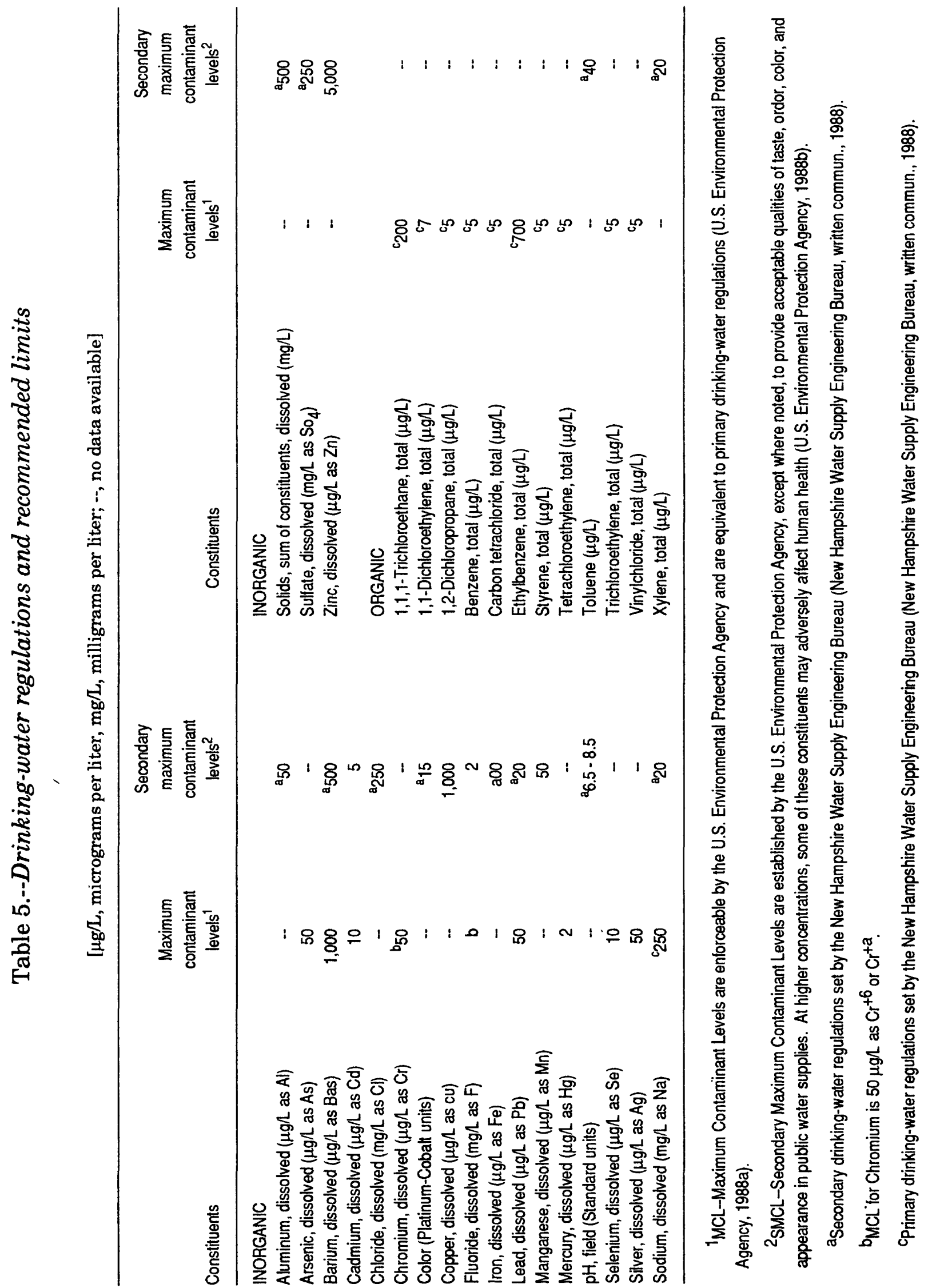




\section{SELECTED REFERENCES}

Anderson-Nichols and Co., Inc., 1980, Ground-water assessment study for $\mathbf{5 0}$ communities in southeastern New Hampshire: U.S. Army Corps of Engineers, New England Division, 2 v., 650 p.

Blackey, F.E., Cotton, J.E., and Toppin, K.W., 1987, Water resources data New Hampshire and Vermont water year 1985: U.S. Geological Survey Water-Data Report NH-VT-85-1, 137 p.

Bradley, Edward, 1964, Geology and ground-water resources of southeastern New Hampshire: U.S. Geological Survey Water-Supply Paper 1695, 80 p.

Bradley, Edward, and Petersen, R. G., 1962, Southeastern area, New Hampshire: U.S. Geological Survey, Open-File Report, New Hampshire basicdata report 1, $53 \mathrm{p}$.

Cotton, J.E., 1977, Availability of ground water in the Piscataqua and other coastal river basins, southeastern New Hampshire: U.S. Geological Survey Water-Resources Investigations Report 77-70, scale: $1: 125,000$.

1987, Ground-water resources of the Lamprey River basin, southeastern New Hampshire: U.S. Geological Survey Water-Resources Investigation Report 84-4252, 53 p.

Earl, F.C., 1983, Surficial geologic map of the Kingston quadrangle Rockingham County, New Hampshire: Concord, N.H., New Hampshire State Department of Resources and Economic Development, Office of State Geologist, scale 1:24,000.

Fishman, M.J. and Friedman, L.C., 1985, Methods for determination of inorganic substances in water and fluvial sediments: U.S. Geological Survey Techniques of Water Resources Investigations, book 5, chap. A1.

Gephart, G.D., 1985a, Surficial geologic map of the Candia quadrangle, Rockingham County, New Hampshire: Concord, N.H., New Hampshire State Department of Resources and Economic Development, Office of State Geologist, scale $1: 24,000$.

$1985 \mathrm{~b}$, Surficial geologic map of the Derry quadrangle, Rockingham County, New Hampshire:
Concord, N.H., New Hampshire State Department of Resources and Economic Development, Office of State Geologist, scale 1:24,000.

1987, Surficial geologic map of the Sandown quadrangle, Rockingham County, New Hampshire: Concord, N.H., New Hampshire State Department of Resources and Economic Development, Office of State Geologist, scale 1:24,000.

Moore, R.B., 1990, Geohydrology and water quality of stratified-drift aquifers in the Exeter, Lamprey, and Oyster River basins, southeastern New Hampshire: U.S. Geological Survey Water-Resources Investigation Report 88-4128, 60 p., 8 pl.

Rantz, S.E. and others, 1982a, Measurement and computation of streamflow: volume 1. Measurement of stage and discharge: U.S. Geological Survey Water-Supply Paper 2175, 284 p.

1982b, Measurement and computation of streamflow: volume 2. Computation of discharge: U.S. Geological Survey Water-Supply Paper $2175,346 \mathrm{p}$.

U.S. Environmental Protection Agency, 1986a, Quality criteria for water: Washington, D.C., Office of Water Regulations and Standards, EPA 440/5-86$001,460 \mathrm{p}$.

1986b, Amendment to national oil and hazardous substance contingency plan; national priorities list; final rule and proposed rules: Federal Register, v. 51, no. 111, p. 21053-21112.

1988a, Maximum contaminant levels (subpart $B$ of 141, National interim primary drinkingwater regulations): U.S. Code of Federal Regulations, Title 40, Parts 100 to 149 , revised as of July 1,1988 , p. $530-533$.

1988b, Secondary maximum contaminent levels (section 143.3 of part 143, National secondary drinking-water regulations): U.S. Code of Federal Regulations, Title 40, Parts 100 to 149, revised as of July 1, 1988, p. 608 .

Weigle, J.M., and Kranes, Richard, 1966, Records of selected wells, springs, test holes, materials tests, and chemical analyses of water in the lower Merrimack River valley in New Hampshire: U.S. Geological Survey Open-File Report, New Hampshire Basic-Data Report 2, 44 p. 